\title{
ON THE FINITE TIME BLOWUP OF THE DE GREGORIO MODEL FOR THE 3D EULER EQUATION
}

\author{
JIAJIE CHEN, THOMAS Y. HOU, AND DE HUANG
}

\begin{abstract}
We present a novel method of analysis and prove finite time self-similar blowup of the original De Gregorio model [7,8] for smooth initial data on the real line with compact support. We also prove self-similar blowup results for the generalized De Gregorio model 23] for the entire range of parameter on $\mathbb{R}$ or $S^{1}$ for Hölder continuous initial data with compact support. Our strategy is to reformulate the problem of proving finite time self-similar singularity into the problem of establishing the nonlinear stability of an approximate self-similar profile using the dynamic rescaling equation. We use the energy method with appropriate singular weight functions and take into account cancellation among various nonlinear terms to extract the inviscid damping effect from the linearized operator around the approximate self-similar profile. We remark that our analysis does not rule out the possibility that the original De Gregorio model is well posed for smooth initial data on a circle. The method of analysis presented in this paper provides a promising new framework to analyze finite time singularity of nonlinear nonlocal systems of partial differential equations.
\end{abstract}

\section{INTRODUCTION}

The three-dimensional Navier-Stokes equations govern the motion of incompressible fluid in the absence of external forcing:

$$
\mathbf{u}_{t}+\mathbf{u} \cdot \nabla \mathbf{u}=-\nabla p+\nu \Delta \mathbf{u}, \quad \nabla \cdot \mathbf{u}=0 .
$$

Here $\mathbf{u}(x, t): \mathbf{R}^{3} \times[0, T) \rightarrow \mathbf{R}^{3}$ is the $3 \mathrm{D}$ velocity vector of the fluid, and $p(x, t): \mathbf{R}^{3} \times[0, T) \rightarrow \mathbf{R}$ describes the scalar pressure. The viscous term $\nu \Delta \mathbf{u}$ models the viscous forcing in the fluid. In the case that $\nu=0$, equations (1.1) are referred to as the Euler equations. The divergence-free condition $\nabla \cdot \mathbf{u}=0$ guarantees the incompressibility of the fluid. The Navier-Stokes equations are among the most fundamental nonlinear partial differential equations. The fundamental question regarding the global regularity of the 3D Euler and Navier-Stokes equations with smooth initial data with finite energy remains open, and it is generally viewed as one of the most important open questions in mathematical fluid mechanics, see the surveys $4,11,12,15$.

Define vorticity $\boldsymbol{\omega}=\nabla \times \mathbf{u}$, then $\boldsymbol{\omega}$ is governed by

$$
\boldsymbol{\omega}_{t}+(\mathbf{u} \cdot \nabla) \boldsymbol{\omega}=\nabla \mathbf{u} \cdot \boldsymbol{\omega}+\nu \Delta \boldsymbol{\omega} .
$$

The term $\nabla \mathbf{u} \cdot \boldsymbol{\omega}$ on the right hand side is referred to as the vortex stretching term, which is absent in the two dimensional case. Note that $\nabla \mathbf{u}$ is formally of the same order as $\boldsymbol{\omega}$. In fact, one can easily show that $\|\nabla \mathbf{u}\|_{L^{p}}$ can be bounded from above and below by $\|\boldsymbol{\omega}\|_{L^{p}}$ for $1<p<\infty$. Thus the vortex stretching term scales quadratically as a function of vorticity, i.e. $\nabla \mathbf{u} \cdot \boldsymbol{\omega} \approx \boldsymbol{\omega}^{2}$. The presence of the vortex stretching term in 3D Navier-Stokes or Euler equations is the main source of difficulty in obtaining global regularity. So far, one can only prove that the 3D Navier-Stokes equations have global smooth solutions for small data.

1.1. The De Gregorio model and its variant. In this paper, we study the finite time singularity of the 1D De Gregorio model [7,8] and its generalization. The De Gregorio model is a simplified $1 \mathrm{D}$ model to study the potential finite time singularity of the $3 \mathrm{D}$ incompressible Euler equations. Specifically, the inviscid De Gregorio model is given below

$$
\omega_{t}+a u \omega_{x}=u_{x} \omega, \quad u_{x}=H \omega,
$$

Date: May 5, 2019. 
where $H$ is the Hilbert transform and $a \in \mathbb{R}$ is a parameter. In this $1 \mathrm{D}$ model, the vorticity vector is modeled as a scalar quantity, $\omega$. The main nonlinear terms, i.e. the advection term, $u \omega_{x}$, and the vortex stretching term, $u_{x} \omega$, are kept in the model. The Biot-Savart law is modeled by $u_{x}=H \omega$, which preserves the same scaling as that of the original Biot-Savart law. The case of $a=0$ is reduced to the well-known Constantin, Lax and Majda model [5], in which the authors proved the finite time singularity formation for a class of initial data. The case $a=1$ was proposed by De Gregorio in [7] and its generalization to $a \in \mathbb{R}$ was proposed by Okamoto et .al. in [23]. Throughout this paper, we call (1.3) the De Gregorio (DG) model. There are various $1 \mathrm{D}$ models proposed in the literature. We refer to [10,20 for excellent surveys of other 1D models for the 3D Euler equations and the surface quasi-geostrophic equation.

One important feature of the De Gregorio model is that it characterizes the competition between the advection term and the vortex stretching term. It is not hard to see that when $a<0$, the advection effect would work together with the stretching effect to produce a singularity. Indeed, Castro and Córdoba [1] proved the finite time blow-up for $a<0$ based on a Lyapunov functional argument. For $a>0$, there are competing nonlocal stabilizing effect due to the advection and the destabilizing effect due to vortex stretching, which are of the same order in terms of scaling. Even for arbitrarily small $a>0$, in which case we expect that the advection effect is much weaker than the vortex stretching, using the same Lyapunov functional argument would fail to prove a finite time singularity since the control of the solution through the Lyapunov functional is not strong enough. We remark that the stabilizing effect of the advection has also been studied by Hou-Li in 17 for an exact 1D model of the 3D axisymmetric Navier-Stokes equations along the symmetry axis and by Hou-Lei for a 3D model of the axisymmetric NavierStokes equations in [16].

The question of whether the De Gregorio model would develop a finite time singularity for $a>0$ has remained unsolved for some time, especially the case of $a=1$. In a recent paper by Elgindi and Jeong [10, they constructed a smooth self-similar profile for small $|a|$ and a $C^{\alpha}$ self-similar profile for all $a \in \mathbb{R}$. We note that the self-similar profiles constructed by Elgindi and Jeong do not have fast decay and the corresponding velocity $u$ has infinite energy, i.e. $\|u\|_{2}=\infty$.

1.2. A novel method of analysis. One of the main contributions of this paper is that we introduce a novel method of analysis that enables us to prove finite time singularity for the De Gregorio model with initial data that have finite energy for the entire range of parameter $a$ both on the real line and on a circle. For small $|a|$, and $a=1$, we can prove finite time self-similar blowup for the $C_{c}^{\infty}$ initial data on the real line. The result for the case of $a=1$ is especially interesting, resolving the open question on the finite time blowup of the original De Gregorio model on the real line. The blowup analysis for the case of $a=1$ with $C_{c}^{\infty}$ initial data is much more challenging than the other blowup results for $|a|$ small or any $a$ with initial data $\omega_{0} \in C_{c}^{\alpha}$ with small Hölder exponent $\alpha$ since the advection term in the case of $a=1$ is comparable to the vortex stretching term and there is strong cancellation between these two competing terms.

Our method of analysis consists of several steps. The first one is to construct an approximate self-similar profile for the De Gregorio model with a small residual in some properly chosen energy norm. In general, it is very difficult to construct a self-similar profile analytically. If the steady state exists and has some regularity, we can compute it numerically with high accuracy to obtain an approximate self-similar profile. The approximation error can be estimated a posteriori. We use this approach to obtain an accurate approximate self-similar profile $\bar{\omega}$ for (1.3) with $a=1$. The second step is to perform linear stability analysis around this approximate self-similar profile by using the dynamic rescaling equation with some appropriately chosen normalization conditions. By designing carefully chosen singular weight functions for both the $L^{2}$ and the $H^{1}$ norms, we are able to extract the crucial inviscid damping effect from the linearized operator around the approximate self-similar profile. The third step is to establish nonlinear stability by choosing an appropriate energy norm and using the bootstrap argument. We can then choose an initial perturbation sufficiently small in the energy norm so that the initial condition of the De Gregorio model has compact support and show that the solution develops a self-similar blowup in finite time. Moreover, we prove that the solution of the dynamic rescaling equation converges to the exact self-similar solution exponentially fast in time in the weighted $L^{2}$ norm. 
By inviscid damping effect, we refer to the property that the eigenvalues of the symmetric part of the linearized operator are all negative. The amount of inviscid damping is characterized by the largest eigenvalue of the symmetric part of the linearized operator, which is negative, but close to zero in absolute value. This relatively weak damping effect makes it extremely difficult to establish linear stability of the linearized operator with a finite amount of damping in the chosen energy norm. We have to develop very sharp estimates for various terms and make use of the isometry property of the Hilbert transform and the cancellation property among various nonlinear terms. This is the most delicate part of the analysis and as a result, our analysis involves many detailed technical computations.

To illustrate the main idea of our analysis, we consider the linear stability of several approximate profiles $\left(\bar{\omega}, \bar{c}_{l}, \bar{c}_{\omega}\right)$ in the dynamical rescaling equation

$$
\omega_{t}+\left(c_{l}(t) x+a u\right) \omega_{x}=\left(c_{\omega}(t)+u_{x}\right) \omega,
$$

where $c_{l}(t)$ and $c_{\omega}(t)$ are scaling parameters. The linearized equation around $\bar{\omega}, \bar{u}, \bar{c}_{l}, \bar{c}_{\omega}$ reads

$$
\omega_{t}+\left(\bar{c}_{l} x+a \bar{u}\right) \omega_{x}=\left(\bar{c}_{\omega}+\bar{u}_{x}\right) \omega+\left(u_{x}+c_{\omega}\right) \bar{\omega}-\left(a u+c_{l} x\right) \bar{\omega}_{x} .
$$

The inviscid damping effect of the system comes from two parts: the stretching term $\left(\bar{c}_{l} x+a \bar{u}\right) \omega_{x}$ and the vortex stretch term $\left(\bar{c}_{\omega}+\bar{u}_{x}\right) \omega$. To extract the inviscid damping effect from the linearized operator, we choose a singular weight $x^{-k}, k \in \mathbb{N}_{+}$to take advantage of the stretching effect. We use the weighted $L^{2}$ estimate of $\omega$ to demonstrate some subtlety of the linear stability analysis:

$$
\frac{1}{2} \frac{d}{d t}\left\langle\omega^{2}, x^{-k}\right\rangle=\left\langle-\left(\bar{c}_{l} x+\bar{u}\right) \omega_{x}+\left(\bar{c}_{\omega}+\bar{u}_{x}\right) \omega, \omega x^{-k}\right\rangle+\left\langle\left(u_{x}+c_{\omega}\right) \bar{\omega}-\left(a u+c_{l} x\right) \bar{\omega}_{x}, \omega x^{-k}\right\rangle
$$

Denote by $I$ the first term on the right hand side. Since $\bar{u}$ has a sublinear growth at infinity, we may assume $\bar{c}_{l} x+\bar{u}=C x$ for some $C>0$. Using integration by parts, we obtain

$$
\begin{aligned}
I & =\left\langle-C x^{-(k-1)} \omega_{x}, \omega\right\rangle+\left\langle\left(\bar{c}_{\omega}+\bar{u}_{x}\right), \omega^{2} x^{-k}\right\rangle \\
& =\left\langle-\frac{C(k-1)}{2}+\left(\bar{c}_{\omega}+\bar{u}_{x}\right), \omega^{2} x^{-k}\right\rangle \triangleq\left\langle D, \omega^{2} x^{-k}\right\rangle .
\end{aligned}
$$

Since $C>0$, we will choose $k$ so that the coefficient $D$ is negative (we choose $k=4$ for $a=1$ and small $|a|$ ). The above weight $x^{-k}$ only reflects the scaling of the singular weight near $x=0$. The actual weight that we use in the analysis is more subtle and we need to take into account the far field behavior of the solution and the approximate self-similar profile.

To estimate the vortex stretch term $\left(u_{x}+c_{\omega}\right) \bar{\omega}$ in (1.5), we take full advantage of the cancellation between $u_{x}$ and $\omega$ (see Lemma A.3). We remark that the $u_{x}$ term is harmless to the stability analysis. For the last term $-\left(a u+c_{l} x\right) \bar{\omega}_{x}$ in (1.5), we will show that it is small if either $|a|$ is small or the profile $\bar{\omega} \in C^{\alpha}$ for small $\alpha>0$. In the case of $a=1$, this term can be controlled by the damping of $\omega$.

The method of analysis that we present in this paper provides a promising new framework to analyze potential finite time singularity of a nonlinear and nonlocal system of partial differential equations. Recently, we have been able to generalize this method of analysis to establish finite time self-similar blowup of the HL model proposed in [18,21] (see also a recent paper in [3]). The HL model is a 1D model for the 3D axisymmetric Euler equations along the boundary. It shares many surprising properties with the original 3D Euler equations and seems to capture very well the essential mechanism that leads to finite time blowup of the $3 \mathrm{D}$ axisymmetric Euler equations reported by Luo-Hou in [21. The analysis of the HL model is much more challenging than that of the De Gregorio model since it is a nonlinear nonlocal system. We are currently working to extend our method of analysis presented in this paper to prove the finite time blowup of the 2D Boussinesq system.

1.3. Main results. We first state the results for (1.3) on $\mathbb{R}$. Our first main result proves the finite time singularity of the original De Gregorio model.

Theorem 1.1. There exist some $C_{c}^{\infty}$ initial data on $\mathbb{R}$ such that the solution of (1.3) with $a=1$ develops an expanding self-similar singularity with blowup scaling $c_{l}=-1$ in finite time.

The second result is finite time blowup of (1.3) for small $|a|$ with $C_{c}^{\infty}$ initial data. 
Theorem 1.2. There exists a positive constant $\delta>0$ such that for $|a|<\delta$ the solution of (1.3) develops a focusing self-similar singularity in finite time for some $C_{c}^{\infty}$ initial data.

The third result is finite time blowup of (1.3) for all $a$ with compact support initial data.

Theorem 1.3. There exists $C_{1}>0$ such that for $\alpha<\min \left(1 / 4, C_{1} /|a|\right)$, the solution of (1.3) with parameter a develops a focusing self-similar singularity in finite time for some $C_{c}^{\alpha}$ initial data.

By expanding or focusing self-similar singularity in Theorems 1.1 1.2 and 1.3, we mean that

$$
\omega(x, t)=(T-t)^{-1} \Omega\left(\frac{x}{(T-t)^{c_{l}}}\right)
$$

is a self-similar solution of (1.3) for some $T>0$ with blowup scaling $c_{l}<0$ in the expanding case and $c_{l}>0$ in the focusing case. The 1/4 upper bound on $\alpha$ in Theorem 1.3 is not a technical assumption but useful for simplifying the presentation. It will be clear in the proof that the results for $\alpha \in[1 / 4,1)$ can be obtained using a similar argument.

The self-similar blowup in Theorem 1.2 and Theorem 1.3 is focusing and these results also hold for the DG model on the circle.

Theorem 1.4. Consider (1.3) on the circle. (1) There exists $C_{1}>0$ such that if $|a|<C_{1}$, the solution of (1.3) develops a self-similar singularity in finite time for some $C_{c}^{\infty}$ initial data. (2) If $\alpha<\min \left(C_{1} /|a|, 1 / 4\right)$, then the solution of (1.3) develops a finite time self-similar singularity for some initial data $\omega_{0} \in C^{\alpha}$ with compact support.

The initial data $\omega_{0}$ we constructed for the previous theorems all satisfy that $\omega_{0}$ is odd and $w_{0} \leq 0$ for $x>0$. The following theorem implies that for $a>0$, the Hölder regularity for $\omega_{0}$ in this class is crucial for the focusing self-similar blow-up.

Theorem 1.5. Suppose that the initial value $\omega_{0}$ is odd and non-positive for $x>0$. There exists a universal positive constant $a_{0}$ such that if $\omega_{0} \in C^{\alpha}$ with compact support and $1 \geq \alpha>a_{0} / a$, then it cannot develop a finite time self-similar singularity with blowup scaling $c_{\omega}=-1, c_{l}>-\alpha^{-1}$.

Theorem 1.3 and the above result show that the critical Hölder exponent for initial datum is $\alpha \approx \frac{1}{a}$ for large positive $a$. In particular, for smooth initial data $\omega \in C^{1}$, it cannot develops a focusing self-similar singularity for $a>C_{1}$ on the circle. For (1.3) on the circle, we can prove stronger results Theorem 4.11 and Proposition 4.14, which will be discussed in Section 4.3.2.

We remark that Theorem 1.1 and Theorem 1.4 do not rule out the possibility that the original De Gregorio model (1.3) with $a=1$ is globally well-posed for smooth initial data on the circle. In fact, Theorem 4.11, Proposition 4.14 and Theorem 5.1 show that it is much harder to obtain finite time blowup for large positive $a$ on a circle than on the real line. In a recent paper by Sverak et. al. [19, they proved the nonlinear stability of the equilibrium $A \sin \left(2\left(\theta-\theta_{0}\right)\right)$ of (1.3) with $a=1$ and $\pi$ periodic, which sheds useful light on the DG model on $S^{1}$. For smooth $\omega_{0}$ with $\omega_{0, x}(0)=0$, the a-priori $L^{1}$ bound for $\omega$ in Proposition 4.14 may provide useful insight on the possible global well-posedness in this class of $\omega$.

Finally, we would like to point out that after we completed our work, we learned from Dr. Elgindi that they have recently established results similar to Theorem 1.2, Theorem 1.3 and Theorem 1.4 independently in 9 .

Organization of the paper. In Section 2, we prove the finite time self-similar blowup of the DG model with small $|a|$. We use this special case as an example to demonstrate the main ideas of our method of analysis to prove finite time self-similar blowup. In Section 3, we construct an accurate approximate profile numerically for the case of $a=1$ and apply our method of analysis to prove the finite time self-similar blowup for $C_{c}^{\infty}$ initial data. In Section 4 we study the case with any $a \in \mathbb{R}$ and prove finite time singularity for any $a \in \mathbb{R}$ on both $\mathbb{R}$ and $S^{1}$. In addition, we prove the criticality of the Hölder continuity on both $\mathbb{R}$ and $S^{1}$. Finally, in Section 5 , we use a Lyapunov functional argument to prove finite time blowup for all $a<0$. In the Appendix, we prove several useful properties of the Hilbert transform, some functional inequalities, and prove 
several Lemmas related to the main results. In the supplementary material [2], we estimate several constants related to the nonlinear stability of the approximate profile for $a=1$.

Notations. Since the functions, e.g. $\omega, u$, have odd-even symmetry, we just need to consider $R^{+}$. The inner product is defined on $R^{+}$, i.e.

$$
\langle f, g\rangle \triangleq \int_{0}^{\infty} f g d x, \quad\|f\|_{L^{p}} \triangleq\left(\int_{0}^{\infty}|f|^{p} d x\right)^{1 / p} .
$$

In Section 3. we further restrict the inner product and the norm on the interval $[0, L]$, e.g $\langle f, g\rangle=\int_{0}^{L} f g d x$, since the support of $\omega, \bar{\omega}$ lies in $[-L, L]$.

We use $C, C_{i}$ to denote absolute constants and $C(A, B, . ., Z)$ to denote constant depending on $A, B, \ldots, Z$. These constants may vary from line to line, unless specified. We also use the notation $A \lesssim B$ if there is some absolute constant $C$ with $A \leq C B$, and denote $A \asymp B$ if $A \lesssim B$ and $B \lesssim A$. We use $\rightarrow$ to denote strong convergence and $\rightarrow$ to denote weak convergence in some norm. The upper bar notation is reserved for the approximate profile, e.g. $\bar{\omega}$. The letters $e, f, a_{1}, a_{2}, a_{3}$ are reserved for some parameters that we will choose in Section 3 .

\section{Finite Time Self-Similar Blowup for Small $|a|$}

In this section, we will present the proof of Theorem 1.2. We use this example to illustrate the main ideas in our method of analysis by carrying stability analysis around an accurate approximate self-similar profile by using a dynamic rescaling formulation.

2.1. Dynamic rescaling formulation. We will prove Theorem 1.2 by using a dynamic rescaling formulation. Let $\omega(x, t), u(x, t)$ be the solutions of the original equation (1.3), then it is easy to show that

$$
\tilde{\omega}(x, \tau)=C_{\omega}(\tau) \omega\left(C_{l}(\tau) x, t(\tau)\right), \quad \tilde{u}(x, \tau)=C_{\omega}(\tau) C_{l}(\tau)^{-1} u\left(C_{l}(\tau) x, t(\tau)\right)
$$

are the solutions to the dynamic rescaling equations

$$
\tilde{\omega}_{\tau}(x, \tau)+\left(c_{l}(\tau) x+a \tilde{u}\right) \tilde{\omega}_{x}(x, \tau)=c_{\omega}(\tau) \tilde{\omega}+\tilde{u}_{x} \omega \quad \tilde{u}_{x}=H \tilde{\omega},
$$

where

$$
C_{\omega}(\tau)=\exp \left(\int_{0}^{\tau} c_{\omega}(s) d \tau\right), C_{l}(\tau)=\exp \left(\int_{0}^{\tau}-c_{l}(s) d s\right), t(\tau)=\int_{0}^{\tau} C_{\omega}(\tau) d \tau .
$$

If there exists $C>0$ such that for any $\tau>0, c_{\omega}(\tau) \leq-C<0$ and the solution $\tilde{\omega}$ is nontrivial, e.g. $\|\tilde{\omega}(\tau, \cdot)\|_{L^{\infty}} \geq c>0$ for all $\tau>0$, we then have

$$
C_{\omega}(\tau) \leq e^{-C \tau}, t(\infty) \leq \int_{0}^{\infty} e^{-C \tau} d \tau=C^{-1}<+\infty,
$$

and that $\left|\omega\left(C_{l}(\tau) x, t(\tau)\right)\right|=C_{\omega}(\tau)^{-1}|\tilde{\omega}(x, \tau)| \geq e^{C \tau}|\tilde{\omega}(x, \tau)|$ blows up at finite time $T=t(\infty)$. If $\left(\tilde{\omega}_{\tau}, c_{l}(\tau), c_{\omega}(\tau)\right)$ converges to a steady state $\left(\omega_{\infty}, c_{l, \infty}, c_{\omega, \infty}\right)$ of (2.2) as $\tau \rightarrow \infty$, one can verify that

$$
\omega(x, t)=\frac{1}{1-t} \omega_{\infty}\left(\frac{x}{(1-t)^{-c_{l, \infty} / c_{\omega, \infty}}}\right)
$$

is a self-similar solution of (1.3).

To simplify our presentation, we will still use $t$ to denote the rescaled time in the rest of the paper.

2.2. Nonlinear stability of the approximate self-similar profile. Consider the dynamical rescaling equation

$$
\begin{aligned}
\omega_{t}+\left(c_{l} x+a u\right) \omega_{x} & =\left(c_{\omega}+u_{x}\right) \omega, \\
u_{x} & =H \omega .
\end{aligned}
$$

For $a=0$, we have an analytic steady state

$$
\omega=\frac{-x}{b^{2}+x^{2}}, \quad u_{x}=\frac{b}{b^{2}+x^{2}}, c_{l}=1, c_{\omega}=-1,
$$


where $b=1 / 2$.

Our strategy in proving the finite-time singularity of the De Gregorio model is the following. We first construct the approximate self-similar profile. Then, we prove the linear stability of the approximate self-similar profile, based on which we can further obtain the nonlinear stability using the bootstrap argument. Finally, we will prove that in the dynamical rescaling formation, the solution of the dynamic rescaling equation converges to the self-similar blowup profile exponentially fast as $\tau \rightarrow \infty$.

Theorem 1.2 is the consequence of the following two Propositions.

Proposition 2.1. There exist $\bar{\omega} \in H^{2}$, two singular weight functions $\varphi$ and $\psi$ and two positive constants, $\mu$ and $a_{0}$ such that if $|a|<a_{0}$ and the initial data $\bar{\omega}+\omega_{0}$ of (2.3) ( $\omega_{0}$ is the initial perturbation) satisfies $\omega_{0} \in H^{2}, \omega_{0, x}(0)=0$ and $E(0)<c|a|$, where

$$
E^{2}(t) \triangleq\left\langle\omega^{2}(t), \varphi\right\rangle+\mu\left\langle\omega_{x}^{2}(t), \psi\right\rangle,
$$

then we have (a) In the dynamical rescaling equation (2.3), the perturbation remains small for all time: $E(t)<c|a|$ for all $t>0$; (b) The physical equation (1.3) with initial data $\bar{\omega}+\omega_{0}$ develops a singularity in finite time.

Proposition 2.2. Suppose that the initial perturbation $\omega_{0} \in H^{2}$ satisfies the assumption in Proposition 2.1. There exists some universal constant $\delta$ with $0<\delta<a_{0}$ such that, if $|a|<\delta$, then the solution of the dynamic rescaling equation (2.3), $\left(\bar{\omega}+\omega, \bar{u}+u, \bar{c}_{\omega}+c_{\omega}, c_{l}+\bar{c}_{l}\right)$, converges to some function, $\omega_{\infty} \in L^{2}(\varphi) \cap H^{1}(\psi), u_{\infty}, c_{l, \infty}>0, c_{\omega, \infty}<0$, in the $L^{2}(\varphi)$ norm exponentially fast. Moreover, $\omega_{\infty}, c_{l, \infty}, c_{\omega, \infty}$ is the steady state of (2.3) in $L^{2}\left(\varphi_{\alpha}\right)$.

In the Appendix, we describe some properties of the Hilbert transform. We will use these properties to estimate the velocity.

Proof of Proposition [2.1. For any $|a| \leq a_{0}$, where $a_{0}>0$ is to be determined. We consider the following approximate self-similar profile:

$$
\begin{aligned}
& \bar{\omega}=\frac{-x}{b^{2}+x^{2}}, \quad \bar{u}_{x}=H \bar{\omega}=\frac{b}{b^{2}+x^{2}}, \quad \bar{u}=\arctan \frac{x}{b}, \\
& \bar{c}_{l}=1-a \bar{u}_{x}(0)=1-2 a, \quad \bar{c}_{\omega}=-1,
\end{aligned}
$$

where $b=1 / 2$. We consider the equation for any perturbation $\omega, u$ around the above approximate self-similar profile

$$
\omega_{t}+\left(\bar{c}_{l} x+a \bar{u}\right) \omega_{x}=\left(\bar{c}_{\omega}+\bar{u}_{x}\right) \omega+\left(u_{x}+c_{\omega}\right) \bar{\omega}-\left(a u+c_{l} x\right) \bar{\omega}_{x}+N(\omega)+F(\bar{\omega}),
$$

where $N$ and $F$ are the nonlinear terms and the error, respectively defined below:

$$
N(\omega)=\left(c_{\omega}+u_{x}\right) \omega-\left(c_{l} x+a u\right) \omega_{x}, \quad F(\bar{\omega})=-a\left(\bar{u}-\bar{u}_{x}(0) x\right) \bar{\omega}_{x} .
$$

We choose the following normalization condition for $c_{l}$ and $c_{\omega}$

$$
c_{l}(t)=-a u_{x}(t, 0), \quad c_{\omega}(t)=-u_{x}(t, 0) .
$$

Note that $\bar{\omega}$ is smooth and the initial data $\omega_{0}+\bar{\omega} \in H^{2}$. Standard local well-posedness results imply that $\omega(t, \cdot)+\bar{\omega}$ remains in $H^{2}$ locally in time, so does $\omega(t, \cdot)$. Using the above normalization condition, the original equation (2.3) and the fact that $\omega, u$ are odd, we can derive the evolution equation for $\omega_{x}(t, 0)$ as follows

$$
\begin{aligned}
\frac{d}{d t}\left(\omega_{x}(t, 0)+\bar{\omega}_{x}(0)\right) & =\left[\left(c_{\omega}+\bar{c}_{\omega}+u_{x}+\bar{u}_{x}\right)(\bar{\omega}+\omega)\right]_{x}-\left.\left[\left(\bar{c}_{l} x+a \bar{u}+c_{l} x+a u\right)\left(\omega_{x}+\bar{\omega}_{x}\right)\right]_{x}\right|_{x=0} \\
& =\left.\left[\left(c_{\omega}+\bar{c}_{\omega}+u_{x}+\bar{u}_{x}\right)-\left(\bar{c}_{l}+c_{l}+a \bar{u}_{x}+a u_{x}\right)\right]\left(\bar{\omega}_{x}+\omega_{x}\right)\right|_{x=0} \\
& =\left.\left[\left(\bar{c}_{\omega}+\bar{u}_{x}\right)-\left(\bar{c}_{l}+a \bar{u}_{x}\right)\right]\left(\bar{\omega}_{x}+\omega_{x}\right)\right|_{x=0}=0,
\end{aligned}
$$

where we have used (2.4) and $\bar{u}_{x}(0)=2$ to obtain the last equality. It follows

$$
\frac{d}{d t} \omega_{x}(t, 0)=\frac{d}{d t}\left(\omega_{x}(t, 0)+\bar{\omega}_{x}(0)\right)=0 \Rightarrow \omega_{x}(t, 0) \equiv \omega_{0, x}(0) .
$$


In the following discussion, our goal is to construct an energy functional $E^{2}(\omega) \triangleq\left\langle\omega^{2}, \varphi\right\rangle+$ $\mu\left\langle\omega_{x}^{2}, \psi\right\rangle$ for some universal constant $\mu$ and show that $E$ satisfies an ODE inequality

$$
\frac{1}{2} \frac{d}{d t} E^{2}(\omega) \leq C E^{3}-(1 / 4-C|a|) E^{2}+C|a| E .
$$

Using a bootstrap argument yields $E(t)<c|a|$ for all time, where $C, c$ are some universal constants.

Linear Stability. Choosing an appropriate singular weight function plays a crucial role in establishing the linear stability. Consider the following weight function

$$
\varphi=-\frac{1}{\bar{\omega} x^{3}}-\frac{1}{b^{2} \bar{\omega} x}=\frac{\left(b^{2}+x^{2}\right)^{2}}{b^{2} x^{4}}
$$

Note that $\varphi$ is singular and is of order $O\left(x^{-4}\right)$ near $x=0$. For an initial perturbation $\omega_{0} \in H^{2}$ that is odd and satisfies $\omega_{0, x}(0)=0, \omega(t, \cdot)$ preserves these properties locally in time (see (2.8)). We will choose $\omega_{0}(x)$ that has $O\left(|x|^{-1}\right.$ ) decay as $|x| \rightarrow \infty$ (same decay as $\bar{\omega}$ ). Hence, $\left\langle\omega^{2}, \varphi\right\rangle$ is finite. We perform the weighted $L^{2}$ estimate

$$
\begin{aligned}
& \frac{1}{2} \frac{d}{d t}\left\langle\omega^{2}, \varphi\right\rangle=\left\langle-\left(\bar{c}_{l} x+a \bar{u}\right) \omega_{x}+\left(\bar{c}_{\omega}+\bar{u}_{x}\right) \omega, \omega \varphi\right\rangle+\left\langle\left(u_{x}+c_{\omega}\right) \bar{\omega}, \omega \varphi\right\rangle \\
& -\left\langle\left(a u+c_{l} x\right) \bar{\omega}_{x}, \omega \varphi\right\rangle+\langle N(\omega), \omega \varphi\rangle+\langle F(\bar{\omega}), \omega \varphi\rangle \triangleq I+I I+I I I+N_{1}+F_{1} .
\end{aligned}
$$

For $I$, we use integration by parts to obtain

$$
I=\left\langle\frac{1}{2 \varphi}\left(\left(\bar{c}_{l} x+a \bar{u}\right) \varphi\right)_{x}+\left(\bar{c}_{\omega}+\bar{u}_{x}\right), \omega^{2} \varphi\right\rangle
$$

Recall $\bar{c}_{l}=1-2 a(2.4)$. Using the explicit formula of profile (2.4) and weight (2.9), we can evaluate the terms in $I$ that do not involve $a$ as follows

$$
\begin{aligned}
& \frac{1}{2 \varphi}(x \varphi)_{x}+\left(\bar{c}_{\omega}+\bar{u}_{x}\right)=\frac{b^{2} x^{4}}{2\left(b^{2}+x^{2}\right)^{2}}\left(\frac{\left(b^{2}+x^{2}\right)^{2}}{b^{2} x^{3}}\right)_{x}+\frac{b}{b^{2}+x^{2}}-1 \\
= & \frac{b^{2} x^{4}}{2\left(b^{2}+x^{2}\right)^{2}}\left(4 \frac{x\left(b^{2}+x^{2}\right)}{b^{2} x^{3}}-3 \frac{\left(b^{2}+x^{2}\right)^{2}}{b^{2} x^{4}}\right)+\frac{b}{b^{2}+x^{2}}-1=\frac{2 x^{2}+b}{x^{2}+b^{2}}-\frac{5}{2}=-\frac{1}{2},
\end{aligned}
$$

where we have used $b=1 / 2$. From (2.4) and (2.9), we have

$$
\begin{aligned}
& \left\|\frac{1}{2 \varphi}\left[\left(\bar{c}_{l} x-x+a \bar{u}\right) \varphi\right]_{x}||_{L^{\infty}}=|a|\right\| \frac{1}{2 \varphi}((-2 x+\bar{u}) \varphi)_{x} \|_{L^{\infty}} \\
\leq & |a||| \frac{-2+\bar{u}_{x}}{2}+\frac{-2 x+\bar{u}}{x} \frac{x \varphi_{x}}{2 \varphi} \|_{L^{\infty}} \leq|a|\left(1+\left\|\bar{u}_{x}\right\|_{\infty}\right)\left(1+\left\|\frac{x \varphi_{x}}{\varphi}\right\|_{\infty}\right) \lesssim|a| .
\end{aligned}
$$

Hence, we can estimate $I$ as follows

$$
I=\left\langle\frac{1}{2 \varphi}\left(\left(\bar{c}_{l} x+a \bar{u}\right) \varphi\right)_{x}+\left(\bar{c}_{\omega}+\bar{u}_{x}\right), \omega^{2} \varphi\right\rangle \leq-\left(\frac{1}{2}-C|a|\right)\left\langle\omega^{2}, \varphi\right\rangle,
$$

for some absolute constant $C$. Denote $\tilde{u} \triangleq u(x)-u_{x}(0) x$. (2.7) implies that

$$
c_{l} x+a u=a \tilde{u}, \quad \tilde{u}_{x}=u_{x}+c_{\omega} .
$$

Using the definition of $I I$ in (2.10), (A.6) and (A.7), we obtain

$$
I I=-\left\langle\left(u_{x}-u_{x}(0)\right) \omega, \frac{1}{x^{3}}+\frac{1}{b^{2} x}\right\rangle=-\frac{\pi}{2 b^{2}} u_{x}^{2}(0) \leq 0 .
$$

For $I I I$, we use the Cauchy-Schwartz inequality to get

$$
I I I=-a\left\langle\tilde{u} \omega, \bar{\omega}_{x} \varphi\right\rangle \leq|a||| \tilde{u} \sqrt{x^{-6}+x^{-4}}||_{2}|| \bar{\omega}_{x}\left(x^{-6}+x^{-4}\right)^{-1 / 2} \varphi \omega \|_{2} .
$$

For $\tilde{u}$, we use the Hardy inequality (A.9) to obtain

$$
\left\langle\tilde{u}^{2}, x^{-6}+x^{-4}\right\rangle \lesssim\left\langle\tilde{u}_{x}^{2}, x^{-4}+x^{-2}\right\rangle \lesssim\left\langle\omega^{2}, x^{-4}+x^{-2}\right\rangle \lesssim\left\langle\omega^{2}, \varphi\right\rangle .
$$

Note that (2.4) and (2.9) implies

$$
\left|\bar{\omega}_{x}\left(x^{-6}+x^{-4}\right)^{-1 / 2} \varphi\right|=\left|\frac{-b^{2}+x^{2}}{\left(b^{2}+x^{2}\right)^{2}} \cdot \frac{x^{3}}{\left(x^{2}+1\right)^{1 / 2}} \cdot \frac{b^{2}+x^{2}}{b x^{2}} \varphi^{1 / 2}\right| \lesssim \varphi^{1 / 2} .
$$


We get

$$
I I I \leq C|a|\left\langle\omega^{2}, \varphi\right\rangle .
$$

Combining the estimates (2.13), (2.14) and (2.17), we obtain

$$
\frac{1}{2} \frac{d}{d t}\left\langle\omega^{2}, \varphi\right\rangle \leq-(1 / 2-C|a|)\left\langle\omega^{2}, \varphi\right\rangle+N_{1}+F_{1} .
$$

Weighted $H^{1}$ estimate. The weighted $H^{1}$ estimate is similar to the $L^{2}$ estimate. We choose the singular weight $\psi$ as follows

$$
\psi=x^{2} \varphi=-\frac{1}{\bar{\omega} x}-\frac{x}{b^{2} \bar{\omega}}=\frac{\left(b^{2}+x^{2}\right)^{2}}{b^{2} x^{2}},
$$

and perform the weighted $H^{1}$ estimate

$$
\begin{aligned}
\frac{1}{2} \frac{d}{d t}\left\langle\omega_{x}^{2}, \psi\right\rangle & =\left\langle-\left(\left(\bar{c}_{l} x+a \bar{u}\right) \omega_{x}\right)_{x}+\left(\left(\bar{c}_{\omega}+\bar{u}_{x}\right) \omega\right)_{x}, \omega_{x} \psi\right\rangle+\left\langle\left(\left(u_{x}+c_{\omega}\right) \bar{\omega}\right)_{x}, \omega_{x} \psi\right\rangle \\
& -\left\langle\left(\left(a u+c_{l} x\right) \bar{\omega}_{x}\right)_{x}, \omega_{x} \psi\right\rangle+\left\langle N(\omega)_{x}, \omega_{x} \psi\right\rangle+\left\langle F(\omega)_{x}, \omega_{x} \psi\right\rangle \\
& \triangleq I+I I+I I I+N_{2}+F_{2} .
\end{aligned}
$$

For $I$, we obtain by using integration by parts that

$$
\begin{aligned}
I & =\left\langle-\left(\bar{c}_{l} x+a \bar{u}\right) \omega_{x x}+\left(-\bar{c}_{l}-a \bar{u}_{x}+\bar{c}_{\omega}+\bar{u}_{x}\right) \omega_{x}+\bar{u}_{x x} \omega, \omega_{x} \psi\right\rangle \\
& =\left\langle\frac{1}{2 \psi}\left(\left(\bar{c}_{l} x+a \bar{u}\right) \psi\right)_{x}+\left(\bar{c}_{\omega}-\bar{c}_{l}+(1-a) \bar{u}_{x}\right), \omega_{x}^{2} \psi\right\rangle-\left\langle\frac{1}{2}\left(\bar{u}_{x x} \psi\right)_{x}, \omega^{2}\right\rangle .
\end{aligned}
$$

Similar to (2.11), we use formula (2.4), (2.19) to evaluate the terms that do not involve $a$.

$$
\begin{aligned}
\frac{1}{2 \psi}(x \psi)_{x}+\left(\bar{c}_{\omega}-1+\bar{u}_{x}\right) & =\frac{b^{2} x^{2}}{2\left(b^{2}+x^{2}\right)^{2}}\left(\frac{\left(b^{2}+x^{2}\right)^{2}}{b^{2} x}\right)_{x}-2+\frac{b}{b^{2}+x^{2}}=-\frac{1}{2}, \\
\left(\bar{u}_{x x} \psi\right)_{x} & =\left(-\frac{2 b x}{\left(b^{2}+x^{2}\right)^{2}} \cdot \frac{\left(b^{2}+x^{2}\right)^{2}}{b^{2} x^{2}}\right)_{x}=\frac{2}{b x^{2}}>0 .
\end{aligned}
$$

Similar to (2.12), we use (2.4) and (2.19) to show that the remaining terms in $I$ are small. We get

$$
\left\|\frac{1}{2 \psi}\left(\left(\bar{c}_{l} x-x+a \bar{u}\right) \psi\right)_{x}-\left(\bar{c}_{l}-1\right)-a \bar{u}_{x}\right\|_{L^{\infty}}=|a||| \frac{1}{2 \psi}((-2 x+\bar{u}) \psi)_{x}+2-\bar{u}_{x} \|_{l^{\infty}} \lesssim|a|,
$$

where we have used $\bar{c}_{l}-1=-2 a$. Therefore, we can estimate $I$ as follows

$$
I \leq-\left(\frac{1}{2}-C|a|\right)\left\langle\omega_{x}^{2}, \psi\right\rangle
$$

where $C$ is some absolute constant. For $I I$, we have

$$
\begin{aligned}
I I & =\left\langle\left(\left(u_{x}+c_{\omega}\right) \bar{\omega}\right)_{x}, \omega_{x} \psi\right\rangle=\left\langle u_{x x} \bar{\omega}, \omega_{x} \psi\right\rangle+\left\langle\left(u_{x}+c_{\omega}\right) \bar{\omega}_{x}, \omega_{x} \psi\right\rangle \\
& =-\left\langle u_{x x} \omega_{x}, \frac{1}{x}+\frac{x}{b^{2}}\right\rangle-\left\langle\tilde{u}_{x}, \omega_{x} \bar{\omega}_{x} \psi\right\rangle \triangleq I I_{1}+I I_{2},
\end{aligned}
$$

where $\tilde{u}=u-u_{x}(0) x, \tilde{u}_{x}=u_{x}-u_{x}(0)$. Note that

$$
u_{x x}=H \omega_{x}, \quad \omega_{x}(0)=u_{x x}(0)=0 .
$$

Applying (A.6) with $\left(u_{x}, \omega\right)$ replaced by $\left(u_{x x}, \omega_{x}\right)$ and (A.8), we obtain

$$
\left\langle u_{x x} \omega_{x}, \frac{1}{x}\right\rangle=0, \quad\left\langle u_{x x} \omega_{x}, x\right\rangle=0 .
$$

It follows that

$$
I I_{1}=-\left\langle u_{x x} \omega_{x}, \frac{1}{x}\right\rangle-\frac{1}{b^{2}}\left\langle u_{x x} \omega_{x}, x\right\rangle=0 .
$$

For $I I_{2}$ in (2.22), we use an argument similar to (2.15) to obtain

$$
\left|I I_{2}\right| \lesssim\left\langle\tilde{u}_{x}^{2}, x^{-4}+x^{-2}\right\rangle^{1 / 2} \cdot\left\langle\left(x^{-4}+x^{-2}\right)^{-1}\left(\bar{\omega}_{x} \psi\right)^{2}, \omega_{x}^{2}\right\rangle^{1 / 2} .
$$


(2.16) shows that this first term in the RHS is bounded by $\left\langle\omega^{2}, \varphi\right\rangle^{1 / 2}$. For the second term, we use the definition (2.4) and (2.19) to obtain

$$
\left|\left(x^{-4}+x^{-2}\right)^{-1}\left(\bar{\omega}_{x} \psi\right)^{2}\right|=\left|\frac{x^{4}}{x^{2}+1}\left(\frac{-b^{2}+x^{2}}{\left(b^{2}+x^{2}\right)^{2}}\right)^{2} \frac{\left(b^{2}+x^{2}\right)^{2}}{b^{2} x^{2}}\right| \psi \lesssim \psi
$$

Hence, we have

$$
I I_{2} \lesssim\left\langle\omega^{2}, \varphi\right\rangle^{1 / 2}\left\langle\omega_{x}^{2}, \psi\right\rangle^{1 / 2} .
$$

For $I I I$ in (2.27), we note that $c_{l} x+a u=a\left(u-u_{x}(0) x\right)$. Similarly, we have

$$
|I I I| \lesssim|a|\left\langle\omega^{2}, \varphi\right\rangle^{1 / 2}\left\langle\omega_{x}^{2}, \psi\right\rangle^{1 / 2} .
$$

In summary, combining (2.21), (2.22), (2.24), (2.25) and (2.26), we prove that

$$
\frac{1}{2} \frac{d}{d t}\left\langle\omega_{x}^{2}, \psi\right\rangle \leq C\left\langle\omega^{2}, \varphi\right\rangle^{1 / 2}\left\langle\omega_{x}^{2}, \psi\right\rangle^{1 / 2}-\left(\frac{1}{2}-C|a|\right)\left\langle\omega_{x}^{2}, \psi\right\rangle+N_{2}+F_{2},
$$

where $C$ is some absolute constant.

Estimate of nonlinear and error terms. We use the following estimate to control $\left\|u_{x}\right\|_{\infty}$

$$
\left\|u_{x}\right\|_{\infty} \leq C\left\|u_{x}\right\|_{2}^{1 / 2}\left\|u_{x x}\right\|_{2}^{1 / 2}=C\|w\|_{2}^{1 / 2}\left\|w_{x}\right\|_{2}^{1 / 2} \leq C\left\langle\omega^{2}, \varphi\right\rangle^{1 / 4}\left\langle\omega_{x}^{2}, \psi\right\rangle^{1 / 4} .
$$

Recall the definition of $N(\omega), F(\bar{\omega})$ in (2.6). For the nonlinear part $N_{1}, N_{2}$, we have

$$
\begin{aligned}
& N_{1}=\langle N(\omega), \omega \varphi\rangle \lesssim(|a|+1)\left\|u_{x}\right\|_{\infty}\left\langle\omega^{2}, \varphi\right\rangle \lesssim\left\|u_{x}\right\|_{\infty}\left\langle\omega^{2}, \varphi\right\rangle, \\
& N_{2}=\left\langle N(\omega)_{x}, \omega_{x} \psi\right\rangle \lesssim(|a|+1)\left\|u_{x}\right\|_{\infty}\left\langle\omega_{x}^{2}, \psi\right\rangle \lesssim\left\|u_{x}\right\|_{\infty}\left\langle\omega_{x}^{2}, \psi\right\rangle,
\end{aligned}
$$

where we use that $|a|<1$ since we only consider small $|a|$ in Theorem 1.2 We note that $F(\bar{\omega})$ (2.6) satisfies $F(\bar{\omega})=O\left(x^{3}\right)$ near 0 and $F(\bar{\omega})=O\left(x^{-1}\right)$ for large $x$. From (2.9) and (2.19), we have $F(\bar{\omega}) \in L^{2}(\varphi)$ and $(F(\bar{\omega}))_{x} \in L^{2}(\psi)$. Then for the error terms $F_{1}, F_{2}$, we can use the Cauchy Schwartz inequality to obtain

$$
\begin{aligned}
& \left|F_{1}\right|=|\langle F(\bar{\omega}), \omega \varphi\rangle| \leq\left\langle F^{2}(\bar{\omega}), \varphi\right\rangle^{1 / 2}\left\langle\omega^{2}, \varphi\right\rangle^{1 / 2} \lesssim|a|\left\langle\omega^{2}, \varphi\right\rangle^{1 / 2} \\
& \left|F_{2}\right|=\left|\left\langle(F(\bar{\omega}))_{x}, \omega_{x} \psi\right\rangle\right| \leq\left\langle(F(\bar{\omega}))_{x}^{2}, \psi\right\rangle^{1 / 2}\left\langle\omega_{x}^{2}, \psi\right\rangle^{1 / 2} \lesssim|a|\left\langle\omega_{x}^{2}, \psi\right\rangle^{1 / 2} .
\end{aligned}
$$

Nonlinear Stability. Let $\mu<1$ be some positive parameter to be determined. We consider the following energy norm

$$
E^{2}(t) \triangleq\left\langle\omega^{2}, \varphi\right\rangle+\mu\left\langle\omega_{x}^{2}, \psi\right\rangle
$$

Using the previous estimates on $u_{x}$ and the Cauchy Schwartz inequality, we have

$$
\left\langle\omega^{2}, \varphi\right\rangle^{1 / 2}\left\langle\omega_{x}^{2}, \psi\right\rangle^{1 / 2} \leq \mu^{-1 / 2} E^{2}, \quad\left\|u_{x}\right\|_{\infty} \leq C\left\langle\omega^{2}, \varphi\right\rangle^{1 / 4}\left\langle\omega_{x}^{2}, \psi\right\rangle^{1 / 4} \leq C \mu^{-1 / 4} E .
$$

Combining (2.18), (2.27), (2.28), (2.29) and the above estimate, we derive

$$
\begin{aligned}
\frac{1}{2} \frac{d}{d t} E^{2}(t) & \leq-\left(\frac{1}{2}-C|a|\right) E^{2}+C \mu\left\langle\omega^{2}, \varphi\right\rangle^{1 / 2}\left\langle\omega_{x}^{2}, \psi\right\rangle^{1 / 2}+C|a| E+C\left\|u_{x}\right\|_{\infty} E^{2} \\
& \leq-\left(\frac{1}{2}-C|a|-C \sqrt{\mu}\right) E^{2}+C|a| E+C \mu^{-1 / 4} E^{3}
\end{aligned}
$$

where $C$ is some absolute constant. Now we choose $\mu$ such that $C \sqrt{\mu}<1 / 4$. Note that $\mu$ is also a universal constant. It follows that

$$
\frac{1}{2} \frac{d}{d t} E^{2}(t) \leq-\left(\frac{1}{4}-C_{1}|a|\right) E^{2}+C_{1}|a| E+C_{1} E^{3},
$$

where $C_{1}$ is a universal constant. For $c_{\omega}(t)$ and $c_{l}(t)$, they satisfy the following estimate

$$
\left|c_{\omega}(t)\right|=\left|u_{x}(t, 0)\right| \leq C_{2} E, \quad\left|c_{l}(t)\right|=\left|a u_{x}(0)\right| \leq C_{2} E,
$$

for some absolute constant. Hence for some small $a_{0}>0$ with $C_{1} a_{0}<1 / 8$ and any $|a|<a_{0}$, we prove by using a bootstrap argument that

$$
E(0)<c|a| \Rightarrow E(t)<c|a| \Rightarrow\left|c_{\omega}(t)\right|,\left|c_{l}(t)\right|<C_{2} E(t)<C_{2} c|a|,
$$


for all $t>0$ and some universal constant $c$. We can further require

$$
a_{0}<\min \left(\frac{1}{8 C_{1}}, \frac{1}{2 C_{2} c}\right) \text {, }
$$

so that we get

$$
\left|c_{\omega}(t)\right|,\left|c_{l}(t)\right|<C_{2} c|a|<1 / 2 \Rightarrow \bar{c}_{\omega}+c_{\omega}(t)<-1 / 2, c_{l}(t)+\bar{c}_{l}>1 / 2 .
$$

As a result, we can choose small initial perturbation $\omega_{0}$ which modifies $\bar{\omega}$ at the far field so that we have an initial data $\bar{\omega}+\omega_{0}$ with compact support. We can also require that $\omega_{0, x}(0)=0$ and $E(0)<c|a|$. Then the bootstrap result and $\bar{c}_{\omega}+c_{\omega}(\tau)<-1 / 2<0$ imply the finite time blowup.

Based on the a-priori estimate, we can further obtain the convergence result.

\subsection{Convergence to the self-similar solution.}

Proof of Proposition 2.2. An important observation is that the approximate self-similar profile is time-independent. Therefore, we take the time derivative in (2.5) to obtain

$$
\omega_{t t}+\left(\bar{c}_{l} x+a \bar{u}\right) \omega_{t x}=\left(\bar{c}_{\omega}+\bar{u}_{x}\right) \omega_{t}+\left(u_{x, t}+c_{\omega, t}\right) \bar{\omega}-\left(a u_{t}+c_{l, t} x\right) \bar{\omega}_{x}+N(\omega)_{t},
$$

where the error term $F(\bar{\omega})$ vanishes since it depends on the approximate self-similar profile only. Note that the normalization condition also implies

$$
\frac{d}{d t} w_{x}(t, 0)=0 .
$$

Exponential convergence. Note that the linearized operator in (2.32) is exactly the same as that in the weighted $L^{2}$ estimate (2.5). Therefore, we obtain

$$
\frac{1}{2} \frac{d}{d t}\left\langle\omega_{t}^{2}, \varphi\right\rangle \leq-(1 / 2-C|a|)\left\langle\omega_{t}^{2}, \varphi\right\rangle+\left\langle N(\omega)_{t}, \omega_{t} \varphi\right\rangle .
$$

The nonlinear part reads

$$
N(\omega)_{t}=\left(c_{\omega, t}+u_{x, t}\right) \omega+\left(c_{\omega}+u_{x}\right) \omega_{t}-\left(c_{l, t} x+a u_{t}\right) \omega_{x}-\left(c_{l} x+a u\right) \omega_{x, t} \triangleq I+I I+I I I+I V .
$$

We are going to show that

$$
\left|\left\langle N(\omega)_{t}, \omega_{t} \varphi\right\rangle\right| \lesssim E(t)\left\langle\omega_{t}^{2}, \varphi\right\rangle
$$

Note that

$$
\begin{aligned}
& \|\omega\|_{\infty},\left\|u_{x}\right\|_{\infty},\left\|\left(u_{x}-u_{x}(0)\right)\left(x^{-4}+x^{-2}\right)^{1 / 2}\right\|_{2} \lesssim E(t), \\
& \left|\frac{u_{t}(x)}{x}\right|=\frac{1}{\pi}\left|\int_{y>0} \log \right| \frac{x+y}{x-y}\left|\frac{1}{x} \omega_{t}(y) d y\right| \lesssim\left\langle\omega_{t}^{2}, \varphi\right\rangle^{1 / 2}\left\langle\left(\log \left|\frac{x+y}{x-y}\right| \frac{1}{x}\right)^{2}, \varphi^{-1}\right\rangle^{1 / 2} \lesssim\left\langle\omega_{t}^{2}, \varphi\right\rangle^{1 / 2}, \\
& \left.\|\left(u_{x, t}-u_{x, t}(0)\right)\left(x^{-4}+x^{-2}\right)^{1 / 2}\right)\left\|_{2},\left|u_{x, t}(0)\right| \lesssim\right\| \omega_{t} \varphi^{1 / 2} \|_{2},
\end{aligned}
$$

where we have used (A.9) with $p=2,4$ to obtain the weighted estimate of $u_{x}-u_{x}(0)$ and $u_{x, t}-u_{x, t}(0)$. The tail behavior of $\varphi(2.9)$ satisfies

$$
\varphi=\frac{b^{2}}{x^{4}}+\frac{2}{x^{2}}+\frac{1}{b^{2}}=O\left(x^{-2}\right)+b^{-2}, \quad \varphi-b^{-2}=\frac{b^{2}}{x^{4}}+\frac{2}{x^{2}}<\varphi .
$$

Recall $\tilde{u}=u-u_{x}(0) x$ and (2.7). We can estimate different parts of $N(\omega)_{t}$ as follows

$$
\begin{aligned}
\left|\left\langle I, \omega_{t} \varphi\right\rangle\right| \leq & \left|\left\langle\left(c_{\omega, t}+u_{x, t}\right) \omega, \omega_{t}\left(\varphi-b^{-2}\right)\right\rangle\right|+b^{-2}\left|\left\langle\left(c_{\omega, t}+u_{x, t}\right) \omega, \omega_{t}\right\rangle\right| \\
\lesssim & \left\langle\tilde{u}_{x, t}^{2},\left(x^{-4}+x^{-2}\right\rangle^{1 / 2}\|\omega\|_{\infty}\left\langle\omega_{t}^{2}, \varphi\right\rangle^{1 / 2}+b^{-2}\right| c_{\omega, t}\|\omega\|_{2}\left\|\omega_{t} \varphi^{1 / 2}\right\|_{2} \\
& \quad+b^{-2}\left\|u_{x, t}||_{2}|| \omega\right\|_{\infty}\left\|\omega_{t}\right\|_{2} \lesssim E(t)\left\langle\omega_{t}^{2}, \varphi\right\rangle, \\
\left\langle I I+I V, \omega_{t} \varphi\right\rangle= & \left.\left\langle c_{\omega}+u_{x}+\frac{\left(\left(c_{l} x+a u\right) \varphi\right)_{x}}{2 \varphi}, \omega_{t}^{2} \varphi\right\rangle\right\rangle \lesssim\left\|u_{x}\right\|_{\infty}\left\langle\omega_{t}^{2}, \varphi\right\rangle \lesssim E(t)\left\langle\omega_{t}^{2}, \varphi\right\rangle, \\
\left\langle I I I, \omega_{t} \varphi\right\rangle= & \left\langle c_{l, t}+a \frac{u_{t}}{x}, \omega_{x} x \varphi^{1 / 2} \omega_{t} \varphi^{1 / 2}\right\rangle \lesssim\left\|c_{l, t}+a \frac{u_{t}}{x}\right\|_{\infty}\left\|\omega_{x} \varphi^{1 / 2} x\right\|_{2}\left\|\omega_{t} \varphi^{1 / 2}\right\|_{2} \\
\lesssim & E(t)\left\langle\omega_{t}^{2}, \varphi\right\rangle,
\end{aligned}
$$


where we have used $\left|x \varphi_{x} / \varphi\right| \lesssim 1$ to estimate $I I+I V$ and $\left\|\omega_{x} \varphi^{1 / 2} x\right\|_{2}=\left\|\omega_{x} \psi^{1 / 2}\right\|_{2} \lesssim E(t)$ to obtain the last inequality. In summary, we have proved (2.34). Consequently, we can rewrite (2.33) as follows

$$
\begin{aligned}
\frac{1}{2} \frac{d}{d t}\left\langle\omega_{t}^{2}, \varphi\right\rangle & \leq-(1 / 2-C|a|)\left\langle\omega_{t}^{2}, \varphi\right\rangle+C_{3} E(t)\left\langle\omega_{t}^{2}, \varphi\right\rangle \\
& \leq-(1 / 2-C|a|)\left\langle\omega_{t}^{2}, \varphi\right\rangle+C_{3} c|a|\left\langle\omega_{t}^{2}, \varphi\right\rangle=-\left(1 / 2-C|a|-C_{3} c|a|\right)\left\langle\omega_{t}^{2}, \varphi\right\rangle
\end{aligned}
$$

for some universal constant $C_{3}$. Thus, there exists $0<\delta<a_{0}$ such that

$$
C \delta+C_{3} c \delta<\frac{1}{4}
$$

Hence, if $|a|<\delta$, we obtain

$$
\frac{d}{d t}\left\langle\omega_{t}^{2}, \varphi\right\rangle \leq-\left(1 / 2-C|a|-C_{3} c|a|\right)\left\langle\omega_{t}^{2}, \varphi\right\rangle \leq-\frac{1}{4}\left\langle\omega_{t}^{2}, \varphi\right\rangle .
$$

It follows that $\left\langle\omega_{t}^{2}, \varphi\right\rangle$ converges to 0 exponentially fast as $t \rightarrow \infty$ and that $\left\langle\omega(t)^{2}, \varphi\right\rangle$ as a sequence of $t$ is Cauchy as $t \rightarrow \infty$. It admits a limit $\omega_{\infty}$ and we have

$$
\left\|\left(\omega(t)-\omega_{\infty}\right) \varphi^{1 / 2}\right\|_{2} \leq e^{-t / 4} .
$$

According to the a-priori estimate $\left\langle\omega_{x}(t, \cdot)^{2}, \psi\right\rangle<E^{2}(t)<(c a)^{2}$, there is a subsequence of $\omega(t)$, which converges to $\omega_{\infty}$ weakly in $H^{1}(\psi)$. Therefore, $\omega_{\infty} \in L^{2}(\varphi) \cap H^{1}(\psi)$.

Convergence to self-similar solution. Finally, we verify that $\omega_{\infty}+\bar{\omega}$ with some $c_{l, \infty}, c_{\omega, \infty}$ is a steady state of (2.3).

We use $\Omega, U, C_{l}, C_{\omega}$ to denote the original solution of (2.3)

$$
\Omega=\omega+\bar{\omega}, U=u+\bar{u}, C_{l}=c_{l}+\bar{c}_{l}, C_{\omega}=c_{\omega}+\bar{\omega} .
$$

In particular, we define $\left(\Omega_{\infty}, U_{\infty}\right)$ by

$$
\Omega_{\infty}=\omega_{\infty}+\bar{\omega}, \quad U_{\infty, x}=H\left(\Omega_{\infty}\right) .
$$

Notice that

$$
\omega_{t}=\Omega_{t}=\left(C_{\omega}+U_{x}\right) \Omega-\left(C_{l} x+a U\right) \Omega_{x} \triangleq K(t) .
$$

Due to the exponential convergence (2.35), we have

$$
\left\langle K(t)^{2}, \varphi\right\rangle \rightarrow 0 \quad \text { as } t \rightarrow+\infty .
$$

Suppose that $\left\{\omega\left(t_{n}, \cdot\right)\right\}_{n>1}$ is a subsequence of $\{\omega(t, \cdot)\}_{t>0}$ such that as $n \rightarrow \infty, t_{n} \rightarrow \infty$ and $\omega\left(t_{n}, \cdot\right)$ converges to $\omega_{\infty}$ weakly in $H^{1}(\psi)$. The strong convergence in $L^{2}(\varphi)$ and the weak convergence in $H^{1}(\psi)$ imply

$$
\begin{gathered}
\Omega\left(t_{n}, \cdot\right)=\omega\left(t_{n}, \cdot\right)+\bar{\omega} \rightarrow \omega_{\infty}+\bar{\omega}=\Omega_{\infty} \quad \text { in } L^{2}(\varphi), \\
\Omega\left(t_{n}, \cdot\right)_{x}=\omega\left(t_{n}, \cdot\right)_{x}+\bar{\omega}_{x} \rightarrow \omega_{\infty, x}+\bar{\omega}_{x}=\Omega_{\infty, x} \quad \text { in } L^{2}(\psi), \\
\Rightarrow x \Omega\left(t_{n}, \cdot\right)_{x} \rightarrow x \Omega_{\infty, x} \quad \text { in } L^{2}(\varphi),
\end{gathered}
$$

where we have used $\psi=x^{2} \varphi$ in the last line. Using the interpolation between $L^{2}(\varphi)$ and $H^{1}(\psi)$, we get the pointwise convergence

$$
U_{x}\left(t_{n}\right) \rightarrow H\left(\Omega_{\infty}\right), \quad \frac{U\left(t_{n}\right)}{x} \rightarrow \frac{U_{\infty}}{x}
$$

in $L^{\infty}$. Recall the normalization condition

$$
c_{\omega}(t)=-u_{x}(t, 0), \quad c_{l}(t)=-a u_{x}(t, 0) .
$$

We get the following convergence

$$
\begin{aligned}
C_{l}\left(t_{n}\right) & =\bar{c}_{l}+c_{l}\left(t_{n}\right)=\bar{c}_{l}-a u_{x}\left(t_{n}, 0\right) \rightarrow \bar{c}_{l}-a\left(U_{x, \infty}(0)-\bar{u}_{x}(0)\right) \triangleq C_{l, \infty}, \\
C_{\omega}\left(t_{n}\right) & =\bar{c}_{\omega}+c_{\omega}(t)=\bar{c}_{\omega}-u_{x}(t, 0) \rightarrow \bar{c}_{\omega}-\left(U_{x, \infty}(0)-\bar{u}_{x}(0)\right) \triangleq C_{\omega, \infty} .
\end{aligned}
$$

Combining the convergence result (2.37), (2.38) and (2.39), we get as $n \rightarrow \infty$

$$
K\left(t_{n}\right)=\left(C_{\omega}+U_{x}\right) \Omega-\left(C_{l}+a \frac{U}{x}\right) x \Omega_{x}
$$


converges weakly in $L^{2}(\varphi)$ to

$$
\left(C_{\omega, \infty}+U_{\infty, x}\right) \Omega_{\infty}-\left(C_{l, \infty}+a \frac{U_{\infty}}{x}\right) x \Omega_{\infty, x}=\left(C_{\omega, \infty}+U_{\infty, x}\right) \Omega_{\infty}-\left(C_{l, \infty} x+a U_{\infty}\right) \Omega_{\infty, x}
$$

Note that (2.36) shows that $K\left(t_{n}\right) \rightarrow 0$ in $L^{2}(\varphi)$. We get

$$
\left(C_{\omega, \infty}+U_{\infty, x}\right) \Omega_{\infty}-\left(C_{l, \infty} x+a U_{\infty}\right) \Omega_{\infty, x}=0
$$

in $L^{2}(\varphi)$. Hence $\Omega_{\infty}, C_{l, \infty}, C_{\omega, \infty}$ is a steady state of (2.3). The a-priori estimate (2.31) and the convergence result imply that $C_{l, \infty}>1 / 2>0, C_{\omega, \infty}<-1 / 2<0$, which further implies that the self-similar singularity is focusing since $C_{l, \infty}>0$. We can rescale the self-similar solution

$$
\left(\Omega_{\infty}, C_{l, \infty}, C_{\omega, \infty}\right) \rightarrow\left(\lambda \Omega_{\infty}, \lambda C_{l, \infty}, \lambda C_{\omega, \infty}\right)
$$

for some $\lambda>0$ so that $C_{\omega}=-1$.

\section{Finite Time Blowup for $a=1$ with $C_{c}^{\infty}$ Initial Data}

In this section, we will prove Theorem 1.1 regarding the finite time self-similar blowup of the original De Gregorio model with $a=1$. Due to the cancellation between the advection term $u \omega_{x}$ and the vortex stretching term, $u_{x} \omega$, finite time singularity of the original De Gregorio model has not been reported in the literature. In this section, we prove a somewhat surprising result, i.e. the original De Gregorio model develops a finite time self-similar blowup solution with compact support. We will use the same method of analysis presented in the previous section except that we do not have an analytic expression of the approximate self-similar profile. We need to rely on numerical computation to obtain an accurate approximate self-similar profile.

To begin with, we consider (1.3) with $a=1$. The associated dynamical rescaling equation reads

$$
\omega_{t}+\left(c_{l} x+u\right) \omega_{x}=\left(c_{\omega}+u_{x}\right) \omega, \quad u_{x}=H \omega .
$$

For initial datum $\omega_{0}$ that is odd and has support in $[-L, L]$, we use the following normalization conditions

$$
c_{l}=-\frac{u(L)}{L}, \quad c_{\omega}=c_{l}
$$

We fix $L=10$. With the above conditions, we have $\left.\left(c_{l} x+u\right)\right|_{x= \pm L}=0$ and

$$
\begin{aligned}
\partial_{t} \omega_{x}(t, 0) & =\left.\partial_{x}\left(\left(u_{x}+c_{\omega}\right) \omega-\left(c_{l} x+u\right) \omega_{x}\right)\right|_{x=0} \\
& =\left(c_{\omega}+u_{x}(t, 0)-c_{l}-u_{x}(t, 0)\right) \omega_{x}(t, 0)=0 .
\end{aligned}
$$

Thus $\omega_{x}(t, 0)$ remains constant and $x= \pm L$ is a stationary point of (3.1) and the support of $\omega$ will remain in $[-L, L]$, as long as the solution of the dynamical rescaling equation remains smooth.

The reader who is not interested in the numerical computation can skip the following discussion on the numerical computation and go directly to Section 3.1.1 and later subsections for the description of the approximate profile and the analysis of linear stability.

3.1. Construction of the approximate self-similar profile. Unlike the case in the previous section where we have an analytic expression of the approximate self-similar profile, we need to approximate the steady state of (3.1) numerically in the case of $a=1$ by using the normalization conditions (3.2). Since $\omega$ is supported on $[-L, L]$ and remains odd for all time, we restrict the computation in the finite domain $[0, L]$ and adopt a uniform discretization with grid points $x_{i}=i h, i=0,1, . ., n=8000, h=L / 8000$. In what follows, the subscript $i$ of $\omega_{i}^{k}$ stands for space discretization, and the superscript $k$ stands for time discretization. We solve (3.1) numerically using the following discretization scheme:

(1) Initial guess is chosen as $\omega_{i}^{0}=-\frac{L-x_{i}}{\pi} \sin \left(\frac{\pi x_{i}}{L}\right), i=0,1, . ., n$. 


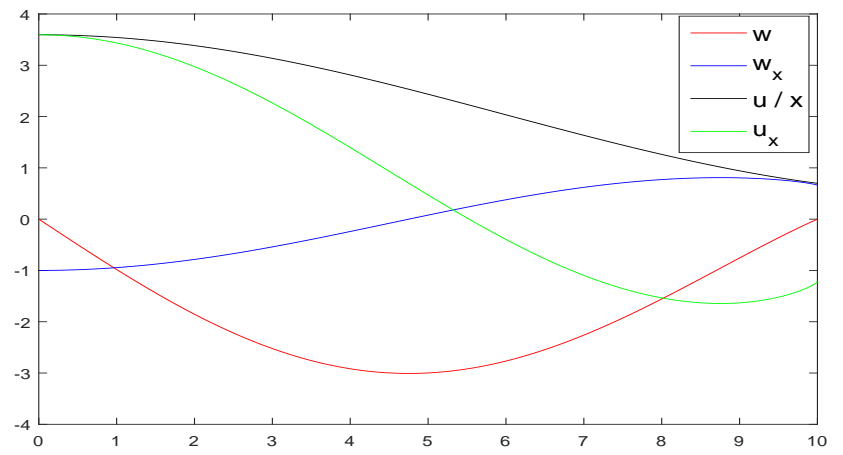

Figure 1. Approximate self-similar profile

(2) The whole function $\omega^{k}$ is obtained from grid point values $w_{i}^{k}$ using a standard cubic spline interpolation on $[-L, L]$, with odd extension of $w^{k}$ on $[-L, 0]$. We approximate $w_{x, i}^{k}$ at the boundary using a second order extrapolation:

$$
w_{x}^{k}(-L)=w_{x}^{k}(L)=w_{x, n}^{k}=\frac{3 \omega_{n}^{k}-4 \omega_{n-1}^{k}+\omega_{n-2}^{k}}{2 h} .
$$

The resulting $\omega^{k}$ is a piecewise cubic polynomial and $\omega^{k} \in C^{2,1}$. The derivative point values $w_{x, i}^{k}$ are evaluated to be $w_{x}^{k}\left(x_{i}\right)$.

(3) Value of $u^{k}$ and $u_{x}^{k}$ at grid points are obtained using the kernel integrals:

$$
u_{i}^{k}=\frac{1}{\pi} \int_{0}^{L} \omega^{k}(y) \log \left|\frac{x_{i}-y}{x_{i}+y}\right| d y, \quad u_{x, i}^{k}=\frac{1}{\pi} \int_{0}^{L} \frac{2 y}{x_{i}^{2}-y^{2}} \omega^{k}(y) d y .
$$

In particular, for each $x_{i}$, the contributions to the above integrals from the neighboring intervals $\left[x_{i-m}, x_{i+m}\right]$ are integrated explicitly using the piecewise cubic polynomial expressions of $\omega$; the contributions from the intervals $[0, L] /\left[x_{i-m}, x_{i+m}\right]$ are approximate by using a piecewise 8-point Legendre-Gauss quadrature, in order to avoid large roundoff error. We choose $m=8$. We have also computed $u_{x x}^{k}$ similarly and will use it later.

(4) The integration in time is performed by the $4_{\text {th }}$ order Runge-Kutta shceme with adaptive time stepping. The discrete time step size $\Delta t_{k}=t_{k+1}-t_{k}$ is given by $\Delta t=\frac{1}{2} \frac{h}{\max _{i}\left|u_{i}\right|}$, respecting the CFL stability condition $|u| \frac{h}{\Delta t} \leq 1$.

(5) After each time step, we apply a local smoothing on $w_{i}^{k}$ to prevent oscillation:

$$
w_{i}^{k} \longleftarrow \frac{1}{4} w_{i-1}^{k}+\frac{1}{2} w_{i}^{k}+\frac{1}{4} w_{i+1}^{k}, \quad i=1, \ldots, n-1 .
$$

Our computation stops when the pointwise residual

$$
F_{\omega, i}^{k}=\left(c_{\omega}^{k}+u_{x, i}^{k}\right) \omega_{i}^{k}-\left(c_{l}^{k} x_{i}+u_{i}^{k}\right) \omega_{x, i}^{k}
$$

satisfies $\max _{i}\left|F_{\omega, i}^{k}\right| \leq 10^{-5}$. Then we use $\bar{\omega}=\omega^{k}$ as our approximate self-similar profile. The corresponding scaling is

$$
\bar{c}_{l}=\bar{c}_{\omega}=-0.6991
$$

by rounding up to 4 significant digits. One can rescale the profile as in (2.40) to obtain an approximate profile with $\bar{c}_{l}=\bar{c}_{\omega}=-1$.

We also perform a convergence test on our numerical scheme. Numerical residuals $F_{w}^{(n)}$ with different choices of $n=500,1000,2000$ are computed at a fixed physical time $T=5$ when the true residual $F_{w}$ (estimated by a much denser mesh) has become negligibly small compared with the discretization error. For each choice of $n$, the $L^{2}$ norm of $F_{w}^{(n)}$ is approximated by the composite trapezoidal rule on a denser mesh of size $N=5 n$. Our numerical experiments show 
that we indeed obtain second order convergence in $L^{2}$ norm of $F_{w}^{(n)}$, which confirms that our numerical scheme is second order accurate.

We will use a computer-assisted proof to establish the finite time self-similar blowup of the original De Gregorio model with $a=1$ by by constructing an accurate approximate selfsimilar profile $\bar{\omega}$. All the numerical computations and quantitative verifications are performed by MATLAB (version 2019a) in the double-precision floating-point operation. To make sure that our computer-assisted proof is rigorous, we adopt the standard method of interval arithmetics (see [22,24]). In particular, we use the MATLAB toolbox INTLAB (version 11 25]) for the interval computations. Every single real number $p$ in Matlab is represented by an interval $\left[p_{l}, p_{r}\right]$ that contains $p$, where $p_{l}, p_{r}$ are precise floating-point numbers of 16 digits. Every computation of real number summation, multiplication or division is performed using the interval arithmetics, and the theoretical outcome $P$ is hence represented by the resulting interval $\left[P_{l}, P_{r}\right]$ that strictly contains $P$. We then obtain a rigorous upper bound on $|P|$ by rounding up $\max \left\{\left|P_{l}\right|,\left|P_{r}\right|\right\}$ to 2 significant digits (or 4 when necessary). We remark that, when encountering a non-essential ill-conditioned computation, especially a division, we will replace it by an alternative well-conditioned one. For example, for some function $f(x)$ such that $f(0)=0, f_{x}(0)<+\infty$, the evaluation of $f(x) / x$ at $x=0$ will be replaced by the evaluation of $f_{x}(0)$.

3.1.1. Compact support of the approximate profile. The approximate profile $\bar{\omega}$ we obtain actually has compact support. Below we explain how we obtain a compactly supported approximate selfsimilar profile. First let us consider the steady state self-similar equation, i.e. taking $\omega_{t}=0$ in (3.1),

$$
\left(c_{l} x+u\right) \omega_{x}=\left(c_{\omega}+u_{x}\right) \omega, \quad u_{x}=H \omega .
$$

Differentiating both sides and then taking $x=0$, we obtain

$$
\left.\left(c_{l}+u_{x}\right) \omega_{x}\right|_{x=0}=\left.\left(c_{\omega}+u_{x}\right) \omega_{x}\right|_{x=0} \Rightarrow c_{l}=c_{\omega},
$$

provided that $\omega_{x}(0) \neq 0$. Suppose that we have a finite time self-similar blowup. Then $c_{\omega}$ must be negative. It follows $c_{l}=c_{\omega}<0$. Since $u>0$ for $x>0$ and grows sublinearly at infinity, there exists $x_{0}>0$ such that

$$
c_{l} x_{0}+u\left(x_{0}\right)=0, \quad c_{l} x+u(x)>0 \text { for } x<x_{0}, \quad c_{l} x+u(x)<0 \text { for } x>x_{0} .
$$

This implies that we expect that the solution of (3.1) will form a shock at $x=x_{0}$. When we solve $\bar{\omega}$ numerically, we fix $x_{0}=L$ by imposing (3.2). Moreover, in (3.1),$c_{\omega}+u_{x}(x)$ is negative for $x>x_{0}$ (see also Figure 1), which leads to damping of $\omega$. For $x>x_{0}$, the transport effect $c_{l} x+u(x)<0$ and the damping effect $c_{\omega}+u_{x}(x)<0$ lead to the compact support of $\omega$ of the steady state solution. For this reason, we choose the initial data with compact support in our computation and the resulting approximate profile also has compact support.

3.1.2. Regularity of the approximate profile. On $[-L, L]$, since $\bar{\omega}$ is obtained from the cubic spline interpolation, it has the regularity $C^{2,1}$. On $\mathbf{R}$, we have $\bar{\omega}( \pm L)=0$. Therefore, $\omega$ is a Lipschitz function on $\mathbf{R}$. We remark that $\bar{\omega}$ is in $H^{1}(\mathbf{R})$ but not in $H^{2}(\mathbf{R})$ since $\bar{\omega}_{x}$ is discontinuous at $x= \pm L$, i.e. $\omega_{x}( \pm L) \neq 0$ (see Figure 1). Multiplying $\left(x^{2}-L^{2}\right.$ ), we get a compactly supported and global Lipschitz function $\left(x^{2}-L^{2}\right) \bar{\omega}_{x}$. Hence we can define the Hilbert transform of $\left(\left(x^{2}-L^{2}\right) \bar{\omega}_{x}\right)_{x}$ which is in $L^{p}$ for any $1 \leq p<+\infty$.

Applying A.5 in Lemma A.2, we have

$$
\bar{u}_{x x}=H \bar{\omega}_{x}, \quad \bar{u}_{x x x}\left(x^{2}-L^{2}\right)=H\left(\bar{\omega}_{x x}\left(x^{2}-L^{2}\right)\right) .
$$

Using the regularity of $\bar{\omega}$, we have that $\bar{u}$ is at least $C^{3}$ in $(-L, L)$ and $\bar{u}_{x x}$ grows logarithmically near $x= \pm L$ since $\bar{\omega}_{x}$ is discontinuous at $x= \pm L$.

3.1.3. Regularity of the perturbation. We will choose initial perturbation $\omega_{0}$ such that $\omega_{0}+\bar{\omega} \in$ $C_{c}^{\infty}$ and $\omega_{0, x}(0)=0$. Standard local well-posedness result shows that $\omega+\bar{\omega}$ remains smooth locally in time. Hence, the regularity of $\omega_{0}$ and $\bar{\omega}$ are the same. Since $\omega$ is odd and $\omega_{x}(0)=0$ (see (3.3)), it is of order $O\left(x^{3}\right)$ near $x=0$. On the other hand, we have $\omega( \pm L)=0$ since its support lies in $[-L, L]$. In the following derivation, the boundary terms when we perform 
integration by parts on $\omega$ terms will vanish, which can be justified by these vanishing conditions. We will use this property without explicitly mentioning it.

3.2. Linear stability of the approximate self-similar profile. Linear stability analysis plays a crucial role in establishing the existence and stability of the self-similar profile. We will establish the linear stability of the approximate self-similar profile in this subsection.

Linearizing (3.1) around $\bar{\omega}, \bar{u}, \bar{c}_{l}, \bar{c}_{\omega}$ yields

$$
\omega_{t}+\left(\bar{c}_{l} x+\bar{u}\right) \omega_{x}=\left(\bar{c}_{\omega}+\bar{u}_{x}\right) \omega+\left(u_{x}+c_{\omega}\right) \bar{\omega}-\left(u+c_{l} x\right) \bar{\omega}_{x}+N(\omega)+F(\bar{\omega}),
$$

where $\omega, u, c_{l}, c_{\omega}$ are the perturbations of the approximate self-similar profile, $N$ and $F$ are the nonlinear terms and the error, respectively

$$
N(\omega)=\left(c_{\omega}+u_{x}\right) \omega-\left(c_{l} x+u\right) \omega_{x}, \quad F(\bar{\omega})=\left(\bar{c}_{\omega}+\bar{u}_{x}\right) \bar{\omega}-\left(\bar{c}_{l} x+\bar{u}\right) \bar{\omega}_{x} .
$$

Main ideas in our linear stability analysis. There are three key observations in our linear stability estimates. First of all, we observe that the $u_{x}$ term (vortex stretch) is harmless to the linear stability analysis as we have shown in Section 2. We construct the weight function carefully to fully exploit the cancellation between $u_{x}$ and $\omega$ (see Lemma A.3). Secondly, note that there is competition between the convection term $u \omega_{x}$ and the vortex stretch term $u_{x} \omega$. We expect some cancellation between their perturbation $u \bar{\omega}_{x}$ and $u_{x} \bar{\omega}$. This enables us to get a smaller constant than that if we apply the Hardy inequality (A.9) to control $u / x$ by $\omega$. Roughly speaking, for $x$ close to $0, u / x$ is of order $\omega / 5$; for $x$ close to $L,(u(x)-u(L)) /(x-L)$ is of order $\omega / 3$. The small constants, $1 / 5$ and $1 / 3$, are crucial for us to obtain the inviscid damping in our linear stability analysis. If we had used a rough estimate by replacing $1 / 5$ by $1 / 2$, we would have failed to obtain the damping effect in the linear stability analysis. Using the first two observations, most interactions can be reduced to some boundary terms. In order to obtain a sharp stability constant, we express these boundary terms as the projection of $\omega$ onto some functions and exploit the cancellation between different projections to obtain the desired linear stability estimate.

For the sake of simplicity, we drop the error term and the nonlinear term in the following derivations. Define a singular weight function on $[-L, L]$

$$
\varphi \triangleq\left(-\frac{1}{x^{3}}-\frac{e}{x}-\frac{f \cdot 2 x}{L^{2}-x^{2}}\right) \cdot\left(\chi_{1}\left(\bar{\omega}-\frac{x \bar{\omega}_{x}}{5}\right)+\chi_{2}\left(\bar{\omega}-\frac{(x-L) \bar{\omega}_{x}}{3}\right)\right)^{-1},
$$

where $\chi_{1}, \chi_{2} \geq 0$ are cutoff functions such that $\chi_{1}+\chi_{2}=1$ and

$$
\chi_{1}(x)=\left\{\begin{array}{ll}
1 & x \in[0,4] \\
0 & x \in[6,10]
\end{array}, \quad \chi_{1}(x)=\frac{\exp \left(\frac{1}{x-4}+\frac{1}{x-6}\right)}{1+\exp \left(\frac{1}{x-4}+\frac{1}{x-6}\right)} \forall x \in[4,6] .\right.
$$

Note that the denominator in (3.6) is positive in $(-L, L) \backslash\{0\}$ and that $\varphi>0$ is a singular weight and is of order $O\left(x^{-4}\right)$ near $x=0, O\left((x-L)^{-2}\right)$ near $x=L$. Denote

$$
\langle f, g\rangle \triangleq \int_{0}^{L} f g d x, \quad\|f\|_{2} \triangleq \sqrt{\langle f, f\rangle} .
$$

For most integrals we consider, it is the same as the integral from 0 to $+\infty$ since the support of $\omega$ lies in $[-L, L]$. Due to the odd-even symmetry, we just need to focus on $[0, L]$. Performing the weighted $L^{2}$ estimate on (3.4) yields

$$
\begin{aligned}
\frac{1}{2} \frac{d}{d t}\left\langle\omega^{2} \varphi\right\rangle & =\left\langle-\left(\bar{c}_{l} x+\bar{u}\right) \omega_{x}+\left(\bar{c}_{w}+\bar{u}_{x}\right) \omega, \omega \varphi\right\rangle+\left\langle\left(u_{x}+c_{\omega}\right), \bar{\omega} \omega \varphi\right\rangle-\left\langle\left(c_{l} x+u\right), \bar{\omega}_{x} \omega \varphi\right\rangle \\
& +\langle N(\omega), \omega \varphi\rangle+\langle F(\bar{\omega}), \omega \varphi\rangle \triangleq D+I+N_{1}+F_{1} .
\end{aligned}
$$

Note that the support of the perturbation lies in $[-L, L]$, the above integral is effectively from 0 to $L$. For $D$, we use integration by parts to obtain

$$
D=\left\langle\frac{1}{2 \varphi}\left(\left(\bar{c}_{l} x+\bar{u}\right) \varphi\right)_{x}+\left(\bar{c}_{w}+\bar{u}_{x}\right), \omega^{2} \varphi\right\rangle \triangleq\left\langle D(\bar{\omega}), \omega^{2} \varphi\right\rangle .
$$


From (3.6), we know that $\varphi(x)=O\left(x^{-4}\right)$ near $x=0$ and $\varphi(x)=O\left((x-L)^{-2}\right)$ near $x=L$. Using these asymptotic properties of $\varphi$, one can obtain that

$$
D(\bar{\omega})(0)=-\left(\bar{c}_{l}+\bar{u}_{x}(0)\right) / 2<0, \quad D(\bar{\omega})(L)=\left(\bar{c}_{l}+\bar{u}_{x}(L)\right) / 2<0 .
$$

We can verify rigorously that $D(\bar{\omega})(x)$ is negative pointwisely. In particular, we treat $\left\langle D(\bar{\omega}), \omega^{2} \varphi\right\rangle$ as a damping term.

We estimate the interaction near $x=0$ and $x=L$ differently. First we split the $I$ term into two terms as follows:

$$
I=\left\langle\left(u_{x}+c_{\omega}\right) \bar{\omega}-\left(c_{l} x+u\right) \bar{\omega}_{x}, \omega \varphi \chi_{1}\right\rangle+\left\langle\left(u_{x}+c_{\omega}\right) \bar{\omega}-\left(c_{l} x+u\right) \bar{\omega}_{x}, \omega \varphi \chi_{2}\right\rangle \triangleq I_{1}+I_{2} .
$$

We use different decompositions of $\left(u_{x}+c_{\omega}\right) \bar{\omega}-\left(c_{l} x+u\right) \bar{\omega}_{x}$ for $x$ close to 0 and to $L$. For $x$ close to 0 (the $\chi_{1}$ part), we use

$$
\begin{aligned}
& \left(u_{x}+c_{\omega}\right) \bar{\omega}-\left(c_{l} x+u\right) \bar{\omega}_{x}=\left(u_{x}+c_{\omega}\right)\left(\bar{\omega}-\frac{1}{5} \bar{\omega}_{x} x\right)+x \bar{\omega}_{x}\left(\frac{1}{5}\left(u_{x}+c_{\omega}\right)-\frac{u+c_{l} x}{x}\right) \\
= & \left(u_{x}+c_{\omega}\right)\left(\bar{\omega}-\frac{1}{5} \bar{\omega}_{x} x\right)+x \bar{\omega}_{x}\left(\frac{1}{5}\left(u_{x}-u_{x}(0)\right)-\frac{u-u_{x}(0) x}{x}-\frac{4}{5}\left(c_{\omega}+u_{x}(0)\right)\right) .
\end{aligned}
$$

For $x$ close to $L$ (the $\chi_{2}$ part), using

$$
c_{\omega}=c_{l}=-u(L) / L \Rightarrow u+c_{l} x=u-u(L)+c_{l}(x-L),
$$

we have

$$
\begin{aligned}
& \left(u_{x}+c_{\omega}\right) \bar{\omega}-\left(c_{l} x+u\right) \bar{\omega}_{x}=\left(u_{x}+c_{\omega}\right) \bar{\omega}-(x-L) \bar{\omega}_{x} \cdot \frac{u-u(L)+c_{l}(x-L)}{x-L} \\
= & \left(u_{x}+c_{\omega}\right)\left(\bar{\omega}-\frac{1}{3} \bar{\omega}_{x}(x-L)\right)+(x-L) \bar{\omega}_{x}\left(\frac{1}{3}\left(u_{x}+c_{\omega}\right)-\frac{u-u(L)+c_{l}(x-L)}{x-L}\right) \\
= & \left(u_{x}+c_{\omega}\right)\left(\bar{\omega}-\frac{1}{3} \bar{\omega}_{x}(x-L)\right)+(x-L) \bar{\omega}_{x}\left(\frac{1}{3}\left(u_{x}-u_{x}(L)\right)-\frac{u-u(L)-u_{x}(L)(x-L)}{x-L}\right) \\
& -\frac{2}{3}(x-L) \bar{\omega}_{x}\left(c_{\omega}+u_{x}(L)\right) .
\end{aligned}
$$

Using (3.9) and the above decompositions near $x=0$, we get

$$
\begin{aligned}
I_{1} & =\left\langle\left(\frac{1}{5} \frac{u_{x}-u_{x}(0)}{x^{2}}-\frac{u-u_{x}(0) x}{x^{3}}\right), x^{3} \bar{\omega}_{x} \omega \varphi \chi_{1}\right\rangle+\left\langle\left(c_{\omega}+u_{x}\right),\left(\bar{\omega}-\frac{1}{5} \bar{\omega}_{x} x\right) \omega \chi_{1} \varphi\right\rangle \\
& -\frac{4}{5}\left(c_{\omega}+u_{x}(0)\right)\left\langle x \bar{\omega}_{x}, \omega \chi_{1} \varphi\right\rangle \triangleq I_{11}+I_{12}+I_{13} .
\end{aligned}
$$

Similarly, near $x=L$, we have

$$
\begin{aligned}
I_{2} & =\left\langle\left(\frac{1}{3} \frac{u_{x}-u_{x}(L)}{x-L}-\frac{u-u(L)-u_{x}(L)(x-L)}{(x-L)^{2}}\right)(x-L)^{2} \bar{\omega}_{x} \omega \varphi \chi_{2}\right\rangle \\
& +\left\langle\left(c_{\omega}+u_{x}\right),\left(\bar{\omega}-\frac{1}{3} \bar{\omega}_{x}(x-L)\right) \omega \varphi \chi_{2}\right\rangle-\frac{2}{3}\left(c_{\omega}+u_{x}(L)\right)\left\langle(x-L) \bar{\omega}_{x}, \omega \varphi \chi_{2}\right\rangle \\
& \triangleq I_{21}+I_{22}+I_{23} .
\end{aligned}
$$

3.2.1. The first part: the interior interaction. To handle the first term on the right hand side of (3.10) and (3.11), i.e. $I_{11}, I_{21}$, we use the Cauchy-Schwartz inequality to obtain

$$
\begin{aligned}
I_{11} & \leq\left\|\left(\frac{1}{5} \frac{u_{x}-u_{x}(0)}{x^{2}}-\frac{u-u_{x}(0) x}{x^{3}}\right)\right\|_{2}\left\|x^{3} \bar{\omega}_{x} \omega \varphi \chi_{1}\right\|_{2}, \\
I_{21} & \leq\left\|\frac{1}{3} \frac{u_{x}-u_{x}(L)}{x-L}-\frac{u-u(L)-u_{x}(L)(x-L)}{(x-L)^{2}}\right\|_{2}\left\|(x-L)^{2} \bar{\omega}_{x} \omega \varphi \chi_{2}\right\|_{2} .
\end{aligned}
$$


ON THE FINITE TIME BLOWUP OF THE DE GREGORIO MODEL FOR THE 3D EULER EQUATION 17

Integration by parts gives

$(3.13)$

$$
\begin{aligned}
& \left\|\left(\frac{1}{5} \frac{u_{x}-u_{x}(0)}{x^{2}}-\frac{u-u_{x}(0) x}{x^{3}}\right)\right\|_{2}^{2} \\
= & \frac{1}{25}\left\|\frac{u_{x}-u_{x}(0)}{x^{2}}\right\|_{2}^{2}-\frac{2}{5} \int_{0}^{L} \frac{\left(u_{x}-u_{x}(0)\right) \cdot\left(u-u_{x}(0) x\right)}{x^{5}} d x+\left\|\frac{u-u_{x}(0) x}{x^{3}}\right\|_{2}^{2} \\
= & \frac{1}{25}\left\|\frac{u_{x}-u_{x}(0)}{x^{2}}\right\|_{2}^{2}-\left.\frac{1}{5} \frac{\left(u-u_{x}(0) x\right)^{2}}{x^{5}}\right|_{0} ^{L}-\frac{1}{5} \cdot 5 \int_{0}^{L} \frac{\left(u-u_{x}(0) x\right)^{2}}{x^{6}} d x+\left\|\frac{u-u_{x}(0) x}{x^{3}}\right\|_{2}^{2} \\
= & \frac{1}{25}\left\|\frac{u_{x}-u_{x}(0)}{x^{2}}\right\|_{2}^{2}-\frac{1}{5 L^{5}}\left(u(L)-u_{x}(0) L\right)^{2}=\frac{1}{25}\left\|\frac{u_{x}-u_{x}(0)}{x^{2}}\right\|_{2}^{2}-\frac{1}{5 L^{3}}\left(c_{\omega}+u_{x}(0)\right)^{2} \\
= & \frac{1}{25}\left\|\frac{\omega}{x^{2}}\right\|_{2}^{2}-\frac{1}{5 L^{3}}\left(c_{\omega}+u_{x}(0)\right)^{2}
\end{aligned}
$$

where we have used $c_{\omega}=c_{l}=-u(L) / L$ in the second to the last line and A.9 with $p=4$ to obtain the last equality. Denote $v \triangleq u-u(L)-u_{x}(L)(x-L)$. Obviously, we have

$$
v(L)=v_{x}(L)=0, v(0)=-u(L)+u_{x}(L) L=L\left(c_{\omega}+u_{x}(L)\right) .
$$

Using the above formula and integration by parts, we obtain

$$
\begin{aligned}
& \left\|\frac{1}{3} \frac{u_{x}-u_{x}(L)}{x-L}-\frac{u-u(L)-u_{x}(L)(x-L)}{(x-L)^{2}}\right\|_{2}^{2}=\left\|\frac{1}{3} \frac{v_{x}}{x-L}-\frac{v}{(x-L)^{2}}\right\|_{2}^{2} \\
= & \frac{1}{9}\left\|\frac{v_{x}}{x-L}\right\|_{2}^{2}-\frac{2}{3} \int_{0}^{L} \frac{v v_{x}}{(x-L)^{3}} d x+\left\|\frac{v}{(x-L)^{2}}\right\|_{2}^{2} \\
= & \frac{1}{9}\left\|\frac{v_{x}}{x-L}\right\|_{2}^{2}-\left.\frac{1}{3} \frac{v^{2}}{(x-L)^{3}}\right|_{0} ^{L}-\frac{1}{3} \cdot 3 \int_{0}^{L} \frac{v^{2}}{(x-L)^{4}} d x+\left\|\frac{v}{(x-L)^{2}}\right\|_{2}^{2} \\
= & \frac{1}{9}\left\|\frac{v_{x}}{x-L}\right\|_{2}^{2}+\frac{1}{3} \frac{v(0)^{2}}{(0-L)^{3}}=\frac{1}{9}\left\|\frac{u_{x}-u_{x}(L)}{x-L}\right\|_{2}^{2}-\frac{1}{3 L}\left(c_{\omega}+u_{x}(L)\right)^{2} .
\end{aligned}
$$

Using a formula similar to A.2 yields

$$
\left(u_{x}-u_{x}(L)\right)(x-L)^{-1}=H\left(\omega(x-L)^{-1}\right) .
$$

We further obtain the following by using the $L^{2}$ isometry of the Hilbert transform

$$
\int_{0}^{L} \frac{\left(u_{x}-u_{x}(L)\right)^{2}}{(x-L)^{2}} d x=\int_{x \in R} \frac{\omega^{2}}{(x-L)^{2}} d x-\int_{x \notin[0, L]} \frac{\left(u_{x}-u_{x}(L)\right)^{2}}{(x-L)^{2}} d x .
$$

Note that the Cauchy-Schwartz inequality implies

$$
\begin{aligned}
& \int_{x \notin[0, L]} \frac{\left(u_{x}-u_{x}(L)\right)^{2}}{(x-L)^{2}} d x \geq \int_{-L}^{0} \frac{\left(u_{x}-u_{x}(L)\right)^{2}}{(x-L)^{2}} d x \\
\geq & \left(\int_{-L}^{0}\left(u_{x}-u_{x}(L)\right) d x\right)^{2}\left(\int_{-L}^{0}(x-L)^{2} d x\right)^{-1} \\
= & \left(u(0)-u(-L)-u_{x}(L) L\right)^{2}\left(\frac{7}{3} L^{3}\right)^{-1}=\frac{3}{7} \frac{\left(c_{\omega}+u_{x}(L)\right)^{2} L^{2}}{L^{3}}=\frac{3}{7} \frac{\left(c_{\omega}+u_{x}(L)\right)^{2}}{L} .
\end{aligned}
$$

Combining (3.14), (3.15) and the above inequality, we get

$$
\begin{aligned}
& \left\|\frac{1}{3} \frac{u_{x}-u_{x}(L)}{x-L}-\frac{u-u(L)-u_{x}(L)(x-L)}{(x-L)^{2}}\right\|_{2}^{2} \\
= & \frac{1}{9} \int_{x \in R} \frac{\omega^{2}}{(x-L)^{2}} d x-\frac{1}{9} \int_{x \notin[0, L]} \frac{\left(u_{x}-u_{x}(L)\right)^{2}}{(x-L)^{2}} d x-\frac{1}{3 L}\left(c_{\omega}+u_{x}(L)\right)^{2} \\
\leq & \frac{1}{9} \int_{x \in R} \frac{\omega^{2}}{(x-L)^{2}} d x-\left(\frac{1}{3 L}+\frac{1}{21 L}\right)\left(c_{\omega}+u_{x}(L)\right)^{2}
\end{aligned}
$$


Combining (3.12), (3.13) and (3.16) and using the elementary inequality $x y \leq \lambda x^{2}+\frac{1}{4 \lambda} y^{2}$, we obtain the estimate for $I_{11}, I_{21}$,

$$
\begin{aligned}
I_{11}+I_{21} & \leq 25 a_{1}\left\|\left(\frac{1}{5} \frac{u_{x}-u_{x}(0)}{x^{2}}-\frac{u-u_{x}(0) x}{x^{3}}\right)\right\|_{2}^{2}+\frac{1}{100 a_{1}}\left\|x^{3} \bar{\omega}_{x} \omega \varphi \chi_{1}\right\|_{2}^{2} \\
& +9 a_{2}\left\|\frac{1}{3} \frac{u_{x}-u_{x}(L)}{x-L}-\frac{u-u(L)-u_{x}(L)(x-L)}{(x-L)^{2}}\right\|_{2}^{2}+\frac{1}{36 a_{2}}\left\|(x-L)^{2} \bar{\omega}_{x} \omega \varphi \chi_{2}\right\|_{2}^{2} \\
& \leq a_{1}\left\|\frac{\omega}{x^{2}}\right\|_{2}^{2}+\frac{1}{100 a_{1}}\left\|x^{3} \bar{\omega}_{x} \omega \varphi \chi_{1}\right\|_{2}^{2}+a_{2} \int_{x \in R} \frac{\omega^{2}}{(x-L)^{2}} d x \\
& +\frac{1}{36 a_{2}}\left\|(x-L)^{2} \bar{\omega}_{x} \omega \varphi \chi_{2}\right\|_{2}^{2}-a_{2}\left(\frac{3}{L}+\frac{3}{7 L}\right)\left(c_{\omega}+u_{x}(L)\right)^{2},
\end{aligned}
$$

where $a_{1}, a_{2}>0$ are some parameters to be chosen later.

3.2.2. The second part. Combining $I_{12}, I_{22}$ in (3.10), (3.11) respectively, and using the definition of $\varphi$ (3.6), we obtain

$$
\begin{aligned}
& I_{12}+I_{22}=\left\langle\left(c_{\omega}+u_{x}\right),\left\{\left(\bar{\omega}-\frac{1}{5} \bar{\omega}_{x} x\right) \chi_{1}+\left(\bar{\omega}-\frac{1}{3} \bar{\omega}_{x}(x-L)\right) \chi_{2}\right\} \omega \varphi\right\rangle \\
= & \left\langle\left(c_{\omega}+u_{x}\right) \omega,\left(-\frac{1}{x^{3}}-\frac{e}{x}-\frac{f \cdot 2 x}{L^{2}-x^{2}}\right)\right\rangle \\
= & \left(c_{\omega}+u_{x}(0)\right)\left\langle\omega,-\frac{1}{x^{3}}-\frac{e}{x}\right\rangle+\left\langle\left(u_{x}-u_{x}(0)\right) \omega,-\frac{1}{x^{3}}-\frac{e}{x}\right\rangle+\left\langle\left(c_{\omega}+u_{x}\right) \omega,-\frac{f \cdot 2 x}{L^{2}-x^{2}}\right\rangle,
\end{aligned}
$$

where $e$ and $f$ are constants in the definition of $\varphi(3.6)$. Since $\omega \in C^{2,1}$ and $\omega(0)=\omega_{x}(0)=$ $\omega_{x x}(0)=0$, we have $\omega \cdot x^{-3} \in L^{1}$ and the above integrals are well-defined. Using (A.6) and (A.7), we obtain

$$
\begin{aligned}
\left\langle\frac{\left(u_{x}-u_{x}(0)\right) w}{x^{3}}\right\rangle & =\frac{1}{2} \int_{\mathbf{R}} \frac{\left(u_{x}-u_{x}(0)\right) w}{x^{3}} d x=0, \\
\left\langle\frac{\left(u_{x}-u_{x}(0)\right) \omega}{x}\right\rangle & =\frac{1}{2} \int_{\mathbf{R}} \frac{\left(u_{x}-u_{x}(0)\right) w}{x} d x=\frac{\pi}{4} u_{x}^{2}(0) .
\end{aligned}
$$

Note that $\left(c_{\omega}+u_{x}\right) \omega$ is odd. The Tricomi identity Lemma A.1 implies

$$
\begin{aligned}
& \left\langle\left(c_{\omega}+u_{x}\right) \omega,-\frac{2 x}{L^{2}-x^{2}}\right\rangle=-\int_{\mathbf{R}^{+}}\left(c_{\omega}+u_{x}\right) \omega\left(\frac{1}{L-x}-\frac{1}{L+x}\right) d x \\
= & -\int_{\mathbf{R}} \frac{\left(c_{\omega}+u_{x}\right) \omega}{L-x} d x=-\pi H\left(\left(c_{\omega}+u_{x}\right) \omega\right)(L)=-\pi c_{\omega} H \omega(L)-\pi H\left(u_{x} \omega\right)(L) \\
= & -\pi c_{\omega} u_{x}(L)-\frac{\pi}{2}\left(u_{x}^{2}(L)-\omega^{2}(L)\right)=-\pi c_{\omega} u_{x}(L)-\frac{\pi}{2} u_{x}^{2}(L) .
\end{aligned}
$$

Combining (3.18), (3.19) and (3.20), we obtain

$$
I_{12}+I_{22}=\left(c_{\omega}+u_{x}(0)\right)\left\langle\omega,\left(-\frac{1}{x^{3}}-\frac{e}{x}\right)\right\rangle-\frac{\pi e}{4} u_{x}^{2}(0)-f \pi c_{\omega} u_{x}(L)-\frac{f \pi}{2} u_{x}^{2}(L) .
$$

3.2.3. The remaining part: the boundary interaction. Let $a_{3} \triangleq a_{2}\left(\frac{3}{L}+\frac{3}{7 L}\right)$. The negative term that appears in the last term of (3.17) can be written as

$$
-a_{2}\left(\frac{3}{L}+\frac{3}{7 L}\right)\left(c_{\omega}+u_{x}(L)\right)^{2}=-a_{3}\left(c_{\omega}+u_{x}(L)\right)^{2} .
$$


ON THE FINITE TIME BLOWUP OF THE DE GREGORIO MODEL FOR THE 3D EULER EQUATION 19

Combining (3.22), (3.21), $I_{13}, I_{23}$ in (3.10) and (3.11), we obtain

$$
\begin{aligned}
& I_{12}+I_{22}+I_{13}+I_{23}-a_{3}\left(c_{\omega}+u_{x}(L)\right)^{2} \\
= & \left(c_{\omega}+u_{x}(0)\right)\left\langle\omega,\left(-\frac{1}{x^{3}}-\frac{e}{x}\right)\right\rangle-\frac{e \pi}{4} u_{x}^{2}(0)-f \pi c_{\omega} u_{x}(L)-\frac{f \pi}{2} u_{x}^{2}(L) \\
& -\frac{4}{5}\left(c_{\omega}+u_{x}(0)\right)\left\langle\omega, x \bar{\omega}_{x} \chi_{1} \varphi\right\rangle-\frac{2}{3}\left(c_{\omega}+u_{x}(L)\right)\left\langle\omega,(x-L) \bar{\omega}_{x} \chi_{2} \varphi\right\rangle-a_{3}\left(c_{\omega}+u_{x}(L)\right)^{2} \\
= & u_{x}(0)\left(\left\langle\omega,\left(-\frac{1}{x^{3}}-\frac{e}{x}\right)-\frac{4}{5} x \bar{\omega}_{x} \chi_{1} \varphi\right\rangle-\frac{e \pi}{4} u_{x}(0)\right) \\
& +c_{\omega}\left(\left\langle\omega,\left(-\frac{1}{x^{3}}-\frac{e}{x}\right)-\frac{4}{5} x \bar{\omega}_{x} \chi_{1} \varphi-\frac{2}{3}(x-L) \bar{\omega}_{x} \chi_{2} \varphi\right\rangle-f \pi u_{x}(L)-a_{3} c_{\omega}\right) \\
& +u_{x}(L)\left(\left\langle w,-\frac{2}{3}(x-L) \bar{\omega}_{x} \chi_{2} \varphi\right\rangle-\frac{f \pi}{2} u_{x}(L)-2 a_{3} c_{\omega}-a_{3} u_{x}(L)\right) .
\end{aligned}
$$

Note that

$$
\begin{aligned}
u_{x}(0) & =-\frac{2}{\pi} \int_{0}^{L} \frac{\omega}{x} d x, \quad u_{x}(L)=\frac{1}{\pi} \int_{0}^{L} \frac{2 x}{L^{2}-x^{2}} \omega d x \\
c_{\omega} & =-\frac{u(L)}{L}=\frac{1}{L \pi} \int_{0}^{L} \log \left(\frac{L+x}{L-x}\right) \omega(x) d x .
\end{aligned}
$$

All the integrals in (3.23) and $c_{\omega}, u_{x}(0), u_{x}(L)$ are the projection of $\omega$ onto some explicit functions. We use the cancellation of these functions to obtain a sharp estimate of the right hand side of (3.23). Denote

$$
\begin{aligned}
g_{c_{\omega}} & \triangleq \frac{1}{L \pi} \log \left(\frac{L+x}{L-x}\right), \quad g_{u_{x}(0)} \triangleq-\frac{2}{\pi x}, \quad g_{u_{x}(L)} \triangleq \frac{2 x}{\pi\left(L^{2}-x^{2}\right)} \\
g_{1} & \triangleq\left(-\frac{1}{x^{3}}-\frac{e}{x}\right)-\frac{4}{5} x \bar{\omega}_{x} \chi_{1} \varphi-\frac{e \pi}{4} g_{u_{x}(0)} \\
g_{2} & \triangleq\left(-\frac{1}{x^{3}}-\frac{e}{x}\right)-\frac{4}{5} x \bar{\omega}_{x} \chi_{1} \varphi-\frac{2}{3}(x-L) \bar{\omega}_{x} \chi_{2} \varphi-f \pi g_{u_{x}(L)}-a_{3} g_{c_{\omega}} \\
g_{3} & \triangleq-\frac{2}{3}(x-L) \bar{\omega}_{x} \chi_{2} \varphi-\left(\frac{f \pi}{2}+a_{3}\right) g_{u_{x}(L)}-2 a_{3} g_{c_{\omega}} .
\end{aligned}
$$

With these notations, we can rewrite (3.23) as follows

$$
\begin{aligned}
& u_{x}(0)\left\langle\omega, g_{1}\right\rangle+c_{\omega}\left\langle\omega, g_{2}\right\rangle+u_{x}(L)\left\langle\omega, g_{3}\right\rangle \\
= & \left\langle\omega, g_{u_{x}(0)}\right\rangle\left\langle\omega, g_{1}\right\rangle+\left\langle\omega, g_{c_{\omega}}\right\rangle\left\langle\omega, g_{2}\right\rangle+\left\langle\omega, g_{u_{x}(L)}\right\rangle\left\langle\omega, g_{3}\right\rangle .
\end{aligned}
$$

For some function $R \in C([0, L]), R>0$ to be chosen, we introduce

$$
\begin{aligned}
& y \triangleq(R \varphi)^{1 / 2} \omega, \quad f_{1} \triangleq(R \varphi)^{-1 / 2} g_{u_{x}(0)}, \quad f_{2} \triangleq(R \varphi)^{-1 / 2} g_{1}, \quad f_{3} \triangleq(R \varphi)^{-1 / 2} g_{c_{\omega}}, \\
& f_{4} \triangleq(R \varphi)^{-1 / 2} g_{2}, \quad f_{5} \triangleq(R \varphi)^{-1 / 2} g_{u_{x}(L)}, \quad f_{6} \triangleq(R \varphi)^{-1 / 2} g_{3} .
\end{aligned}
$$

Our goal is to find the best constant of the following inequality for any $\omega \in L^{2}(\varphi)$

$$
\left\langle f_{1}, y\right\rangle\left\langle f_{2}, y\right\rangle+\left\langle f_{3}, y\right\rangle\left\langle f_{4}, y\right\rangle+\left\langle f_{5}, y\right\rangle\left\langle f_{6}, y\right\rangle \leq C_{o p t}\|y\|_{2}^{2}
$$

which is equivalent to

$$
\left\langle\omega, g_{u_{x}(0)}\right\rangle\left\langle\omega, g_{1}\right\rangle+\left\langle\omega, g_{c_{\omega}}\right\rangle\left\langle\omega, g_{2}\right\rangle+\left\langle\omega, g_{u_{x}(L)}\right\rangle\left\langle\omega, g_{3}\right\rangle \leq C_{o p t}\left\langle R, \omega^{2} \varphi\right\rangle,
$$

so that we can bound (3.25) by $\left\langle R, \omega^{2} \varphi\right\rangle$ as sharp as possible. From the definition of functions $g, f$, we have that

$$
g_{3} \in \operatorname{span}\left(g_{c_{\omega}}, g_{u_{x}(0)}, g_{u_{x}(L)}, g_{1}, g_{2}\right) \Rightarrow f_{6} \in \operatorname{span}\left(f_{1}, f_{2}, \ldots, f_{5}\right) \triangleq V, \quad \operatorname{dim} V=5 .
$$

Without loss of generality, we assume $y \in V$ since $\|P y\|_{2} \leq\|y\|_{2}$ and $\left\langle y, f_{i}\right\rangle=\left\langle P y, f_{i}\right\rangle$, where $P$ is the orthogonal projector onto $V$. Suppose that $\left\{e_{i}\right\}_{i=1}^{5}$ is an orthonormal basis (ONB) in $V$ with respect to the $L^{2}$ inner product on $[0, L]$. It can be obtained via the GramSchmidt procedure. Then we have $z=\sum_{i=1}^{5}\left\langle z, e_{i}\right\rangle e_{i}$ for any $z \in V$. We consider the linear 
map $T: V \rightarrow R^{5}$ defined by $(T z)_{i}=\left\langle z, e_{i}\right\rangle, \forall z \in V$. It is obvious that $T$ is a linear isometry from $\left(V,\langle\cdot, \cdot\rangle_{L^{2}}\right)$ to $R^{5}$ with the Euclidean inner product, i.e. $\|T z\|_{L^{2}}=\|z\|_{L^{2}}$. Denote $v=T y, v_{i}=T f_{i} \in R^{5}$. Using the linear isometry, i.e. $\left\langle f_{i}, y\right\rangle=v^{T} v_{i}$ and $\|y\|_{2}^{2}=v^{T} v$, we can reduce (3.27) to

$$
\sum_{1 \leq i \leq 3}\left(v^{T} v_{2 i-1}\right)\left(v_{2 i}^{T} v\right) \leq C_{o p t} v^{T} v \Longleftrightarrow v^{T}\left(\sum_{1 \leq i \leq 3} v_{2 i-1} v_{2 i}^{T}\right) v \leq C_{o p t} v^{T} v .
$$

Denote $M \triangleq \sum_{1 \leq i \leq 3} v_{2 i-1} v_{2 i}^{T} \in R^{5 \times 5}$. Using the fact that $v^{T} M v=v^{T} M^{T} v$, we can symmetrize (3.28) to obtain

$$
v^{T} M v \leq C_{o p t} v^{T} v \Longleftrightarrow v^{T} \frac{M+M^{T}}{2} v \leq C_{o p t} v^{T} v .
$$

Since $\left(M^{T}+M\right) / 2$ is symmetric, the optimal constant $C_{o p t}$ is the maximal eigenvalue of $(M+$ $\left.M^{T}\right) / 2$, i.e.

$$
C_{\text {opt }}=\lambda_{\max }\left(\frac{M+M^{T}}{2}\right)=\lambda_{\max }\left(\frac{1}{2} \sum_{1 \leq i \leq 3}\left(v_{2 i-1} v_{2 i}^{T}+v_{2 i} v_{2 i-1}^{T}\right)\right) .
$$

We remark that maximal eigenvalue $\lambda_{\max }$ is independent of the choice of the ONB of $V$. For other ONB, the resulting $\lambda_{\max }$ will be $\lambda_{\max }\left(Q\left(M+M^{T}\right) Q^{T} / 2\right)$ for some orthonormal matrix $Q \in R^{5 \times 5}$, which is the same as (3.29). Using (3.23), (3.25), (3.27) and (3.29), we have proved

$$
I_{12}+I_{22}+I_{13}+I_{23}-a_{3}\left(c_{\omega}+u_{x}(L)\right)^{2} \leq \lambda_{\max }\left(\frac{1}{2} \sum_{1 \leq i \leq 3}\left(v_{2 i-1} v_{2 i}^{T}+v_{2 i} v_{2 i-1}^{T}\right)\right)\left\langle R, \omega^{2} \varphi\right\rangle,
$$

where $v_{i} \in R^{5}$ is the coefficient of $f_{i}$ (see (3.26) ) expanded under an ONB $\left\{e_{i}\right\}_{i=1}^{5}$ of $V=$ $\operatorname{span}\left(f_{1}, f_{2}, . ., f_{5}\right)$, i.e. the $j$-th component of $v_{i}$ satisfies $v_{i j}=\left\langle f_{i}, e_{j}\right\rangle$. We will choose $R$ so that $\lambda_{\max }<1$ and then the left hand side can be controlled by $\left\langle R, \omega^{2} \varphi\right\rangle$.

3.2.4. Summary of the estimates. In summary, we collect all the estimates of $I_{i j}, i=1,2, j=$ $1,2,3$, (3.10), (3.11), (3.17) and (3.30) to conclude

$$
\begin{aligned}
& \left\langle\left(u_{x}+c_{\omega}\right) \bar{\omega}-\left(c_{l} x+u\right), \bar{\omega}_{x}, \omega \varphi\right\rangle=I=I_{1}+I_{2}=\sum_{i=1,2, j=1,2,3} I_{i j} \\
\leq & a_{1}\left\|\frac{\omega}{x^{2}}\right\|_{2}^{2}+\frac{1}{100 a_{1}}\left\|x^{3} \bar{\omega}_{x} \omega \varphi \chi_{1}\right\|_{2}^{2}+a_{2} \int_{x \in R} \frac{\omega^{2}}{(x-L)^{2}} d x \\
+ & \frac{1}{36 a_{2}}\left\|(x-L)^{2} \bar{\omega}_{x} \omega \varphi \chi_{2}\right\|_{2}^{2}+\lambda_{\max }\left(\frac{1}{2} \sum_{1 \leq i \leq 3}\left(v_{2 i-1} v_{2 i}^{T}+v_{2 i} v_{2 i-1}^{T}\right)\right)\left\langle R, \omega^{2} \varphi\right\rangle \\
\triangleq & \left\langle A(\bar{\omega}), \omega^{2} \varphi\right\rangle+\lambda_{\max }\left(\frac{1}{2} \sum_{1 \leq i \leq 3}\left(v_{2 i-1} v_{2 i}^{T}+v_{2 i} v_{2 i-1}^{T}\right)\right)\left\langle R, \omega^{2} \varphi\right\rangle,
\end{aligned}
$$

where $A(\bar{\omega})$ is the sum of the four terms in the first inequality and is given by

$$
A(\bar{\omega})=\left(\frac{a_{1}}{x^{4}}+\frac{a_{2}}{(x-L)^{2}}+\frac{a_{2}}{(x+L)^{2}}\right) \varphi^{-1}+\frac{\left(x^{3} \bar{\omega}_{x} \chi_{1}\right)^{2} \varphi}{100 a_{1}}+\frac{\left((x-L)^{2} \bar{\omega}_{x} \chi_{2}\right)^{2} \varphi}{36 a_{2}} .
$$

Optimizing the parameters. To optimize the estimate, we choose

$$
e=0.005, f=0.004, a_{1}=\frac{1}{6}, a_{2}=1.4 f=0.0056, a_{3}=a_{2}\left(3+\frac{3}{7}\right) / L=0.00192 .
$$

After specifying these parameters, the damping term $D(\bar{\omega})$ (see (3.7)) and the estimate of the interior interaction $A(\bar{\omega})$ are completely determined. Then we choose

$$
R(\bar{\omega})=-D(\bar{\omega})-A(\bar{\omega})-0.3
$$

in (3.26). The numerical values of $D(\bar{\omega}), A(\bar{\omega})$ and $R(\bar{\omega})$ on the grid points are plotted in the first subfigure in Figure 2. We can verify rigorously (see the discussion below) that $R(\bar{\omega})=$ $-D(\bar{\omega})-A(\bar{\omega})-0.3>0$. In particular, the damping term satisfies $D(\bar{\omega})<-0.3-A(\bar{\omega})$ and is 

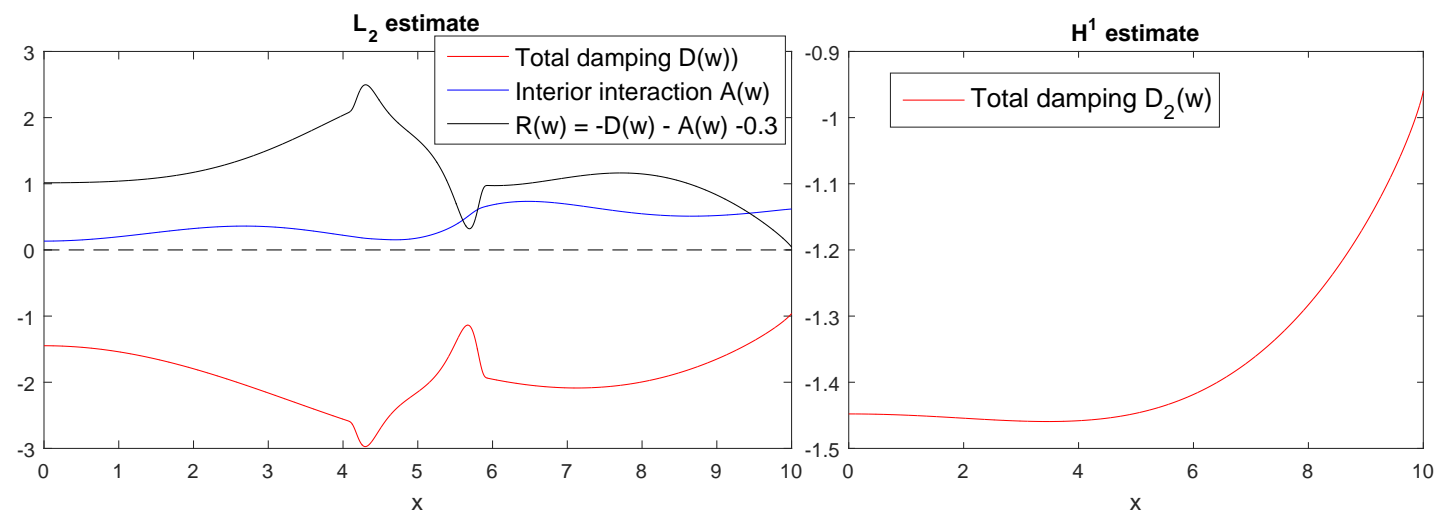

Figure 2. Left: Total damping $D(\bar{\omega})$ in the $L^{2}$ estimate, the estimate of the interior interaction $A(\bar{\omega})$ and the remaining terms $R(\bar{\omega})$. Right: Total damping in the $H^{1}$ estimate. Here, the mesh size is $h=2.5 \cdot 10^{-5}$.

negative pointwisely. The corresponding $f_{i}$ in (3.27) are determined. The optimal constant in (3.30) can be computed :

$$
C_{\text {opt }}=\lambda_{\max }\left(\frac{1}{2} \sum_{1 \leq i \leq 3}\left(v_{2 i-1} v_{2 i}^{T}+v_{2 i} v_{2 i-1}^{T}\right)\right)<1 .
$$

Combining $\left\langle D(\bar{\omega}), \omega^{2} \varphi\right\rangle$ in (3.7), (3.31) and (3.33), we obtain the linear estimate

$$
\begin{aligned}
& \frac{1}{2} \frac{d}{d t}\left\langle\omega^{2}, \varphi\right\rangle=\left\langle D(\bar{\omega}), \omega^{2} \varphi\right\rangle+I+N_{1}+F_{1} \\
\leq & \left\langle D(\bar{\omega}), \omega^{2} \varphi\right\rangle+\left\langle A(\bar{\omega}), \omega^{2} \varphi\right\rangle+\left\langle R(\bar{\omega}), \omega^{2} \varphi\right\rangle+N_{1}+F_{1}=-0.3\left\langle\omega^{2}, \varphi\right\rangle+N_{1}+F_{1} .
\end{aligned}
$$

For those who are not interested in the rigorous verification of the numerical values, they can skip the following discussion and jump to Section 3.3 for the weighted $H^{1}$ estimate.

Rigorous verification of the numerical values. We remark that all the estimates, except for $R(\bar{\omega})>0, C_{o p t}<1$ (3.33) and $D_{2}(\bar{\omega})<-0.95$ (3.38) to be discussed later, are valid independent of the profile. We will use the following strategy to prove that $R(\bar{\omega})>0, C_{\text {opt }}<1$, and $D_{2}(\bar{\omega})<-0.95$.

(a) Obtaining an explicit approximate self-similar profile. As described in section 3.1. our approximate self-similar profile $\bar{\omega}$ is expressed in terms of a piece-wise cubic polynomial over the grid points $x_{i}=\frac{i L}{n}, i=0, \cdots, n$. The coefficients of the corresponding cubic Hermite basis functions, $\bar{\omega}\left(x_{i}\right), \bar{\omega}_{x}\left(x_{i}\right)$, are computed accurately up to double-precision, and will be represented in the computations using the interval arithmetics with exact floating-point bounding intervals. All the following computer-assisted estimates are based on the rigorous interval arithmetics.

(b) Accurate point values of $\bar{u}, \bar{u}_{x}, \bar{u}_{x x}$. With the explicit expression of $\bar{\omega}$, the value of $\bar{u}_{x}(x)$ (or $\bar{u}(x), \bar{u}_{x x}(x)$ ) at any point $x$ can be obtained accurately from certain integrals involving $\bar{w}$ on $[-L, L]$, using a high order numerical quadrature with rigorously controllable error. In particular, our numerical approximation of these integrals is a mixture of analytic integration and the composite 8-point Legendre-Gauss quadrature. For any $x \in[0, L]$, the integral contribution to $u_{x}(x)$ from mesh intervals within $m=8$ mesh points is computed explicitly and accurately up to double-precision, i.e. $10^{-15}$; the piecewise integral error from the Legendre-Gauss quadrature on the intermediate domains, which are $8 h$ to $O(1)$ distance away from $x$, can be bounded by $\operatorname{ch} 8^{-2 \times 8}=\operatorname{ch} 8^{-16}$ for some uniform constant $c<1$, due to the singular factor $\frac{1}{x-y}$ in the integrand; the piecewise integral error from the domains farther away $(O(1)$ distance to $x)$ can be bounded by $c h * h^{16}$ by a standard Legendre-Gauss quadrature error estimate. Therefore, the overall error of each point value $\bar{u}_{x}(x)$ can be rigorously bounded by $\epsilon=\max \left\{10^{-15}, L c 8^{-16}, L c h^{16}\right\}$, which will be taken into account in its interval representations. That is, each $\bar{u}(x)$ will be represented by $\left[\left\lfloor\bar{u}_{x}(x)-\epsilon\right\rfloor_{f},\left[\bar{u}_{x}(x)+\epsilon\right\rceil_{f}\right]$ in any computation using the interval arithmetics, where $\lfloor\cdot\rfloor_{f}$ and $\lceil\cdot]_{f}$ stand for the rounding down and rounding up to 
the nearest floating-point value, respectively. We remark that we will need the values of $\bar{u}_{x}(x)$ at finitely many points only. The same arguments apply to $u(x)$ and $u_{x x}(x)$ as well.

(c) Estimates of some (weighted) norms of $\bar{\omega}, \bar{u}$. Since the profile $\bar{\omega}$ is a piecewise cubic polynomial with accurate coefficients, we can estimate some norms of $\bar{\omega}$, e.g. $\left\|\bar{\omega}_{x}\right\|_{L^{\infty}},\left\|\bar{\omega}_{x x}\right\|_{L^{\infty}}$, accurately. From the discussion of the regularity of $\bar{u}, \bar{\omega}$ in Section (3.1.2), the regularity of $\bar{u}$, e.g. $\left\|\bar{u}_{x}\right\|_{\infty},\left\|\bar{u}_{x x}\right\|_{L^{2}}$, can be bounded by some norm of $\bar{\omega}$. Therefore, we can approximate functions involving $\bar{u}, \bar{\omega}$, such that the weight $\varphi, A(\bar{\omega})$ and $D(\bar{\omega})$, by their values on the grid points up to an error that can be controlled using the smoothness of these functions.

(d) Rigorous and accurate estimates of certain integrals. To obtain the integral of some function $P$, we use the composite Trapezoidal rule

$$
\int_{a h}^{b h} P(x) d x=\sum_{a \leq i<b}\left(P\left(x_{i}\right)+P\left(x_{i+1}\right)\right) h / 2+\operatorname{error}(P) .
$$

The composite Trapezoidal rule uses the values of $P$ on the grid points only, which can be obtained up to the round off error. The integral error, error $(P)$, can be bounded by the $L^{1}$ norm of its first or second order derivative, i.e. $C\left\|P^{\prime \prime}\right\|_{L^{1}} h^{2}$ or $C\left\|P^{\prime}\right\|_{L^{1}} h$ for some absolute constant $C$. We use this approach to obtain the coefficients $v_{i}$ in (3.30) that involve the inner product of $f_{i}$ (3.26).

(e) Rigorous estimates of the eigenvalues. The optimal constant $C_{\text {opt }}(3.33)$ is the maximal eigenvalue of a symmetric matrix $S \in R^{5 \times 5}$. Suppose that $S$ is the exact matrix and $\hat{S}$ is the matrix that we compute numerically. Note that $\hat{S}$ differs from $S$ only due to the approximation of the inner product by the composite Trapezoidal rule to obtain the entries $v_{i}$ in (3.33). In particular, we have $\|S-\hat{S}\|_{L^{1}}=O\left(h^{2}\right)$, where $\|\cdot\|_{L^{1}}$ is the matrix $L^{1}$ norm. We perform the eigendecomposition of $\hat{S}$ numerically and obtain $Q \Lambda Q^{T}$, where $\Lambda$ is a diagonal matrix containing the eigenvalues. The numerical result shows that $\max _{i} \Lambda_{i i}<0.92$. Finally, we use the perturbation theorem of eigenvalues of a symmetric matrix (see e.g. [13]) to obtain

$$
\left|\lambda_{\max }(S)-\lambda_{\max }(\hat{S})\right| \leq\|S-\hat{S}\|_{L^{1}} \leq C\left(f_{i}\right)\left(h^{2}\right), \quad\left|\lambda_{\max }(\hat{S})-\max _{i} \Lambda_{i i}\right| \leq\left\|Q \Lambda Q^{T}-\hat{S}\right\|_{L^{1}}
$$

to verify that

$C_{\text {opt }}=\lambda_{\max }(S) \leq \max _{i} \Lambda_{i i}+\|S-\hat{S}\|_{L^{1}}+\left\|Q \Lambda Q^{T}-\hat{S}\right\|_{L^{1}}<0.92+C\left(f_{i}\right) h^{2}+\left\|Q \Lambda Q^{T}-\hat{S}\right\|_{L^{1}}<1$,

where the constant $C\left(f_{i}\right)$ can be bounded by some norm of $\bar{\omega}$ and the smallness of $\left\|Q \Lambda Q^{T}-\hat{S}\right\|_{L^{1}}$ can be verified a posteriori, which is at the order of double-precision, i.e. $10^{-15}$.

3.3. Weighted $H^{1}$ estimate. We choose

$$
\psi=-\frac{1}{\bar{\omega}}\left(\frac{1}{x}-\frac{x}{L^{2}}\right), \quad x \in[0, L],
$$

as the weight for the weighted $H^{1}$ estimate. Note that the weight $\psi$ is nonnegative for $0 \leq x \leq L$. Then we can perform the weighted $H^{1}$ estimate as follows

$$
\begin{aligned}
\frac{1}{2} \frac{d}{d t}\left\langle\omega_{x}^{2}, \psi\right\rangle & =\left\langle-\left(\left(\bar{c}_{l} x+\bar{u}\right) \omega_{x}\right)_{x}+\left(\left(\bar{c}_{\omega}+\bar{u}_{x}\right) \omega\right)_{x}, \omega_{x} \psi\right\rangle+\left\langle\left(\left(u_{x}+c_{\omega}\right) \bar{\omega}\right)_{x}, \omega_{x} \psi\right\rangle \\
& -\left\langle\left(\left(u+c_{l} x\right) \bar{\omega}_{x}\right)_{x}, \omega_{x} \psi\right\rangle+\left\langle N(\omega)_{x}, \omega_{x} \psi\right\rangle+\left\langle F(\bar{\omega})_{x}, \omega_{x} \psi\right\rangle \\
& \triangleq I+I I+I I I+N_{2}+F_{2} .
\end{aligned}
$$

For $I$, we use $\bar{c}_{l}=\bar{c}_{\omega}$ and integration by parts to get

$$
\begin{aligned}
I & =\left\langle-\left(\bar{c}_{l} x+\bar{u}\right) \omega_{x x}+\bar{u}_{x x} \omega, \omega_{x} \psi\right\rangle \\
& =\left\langle\frac{1}{2 \psi}\left(\left(\bar{c}_{l} x+\bar{u}\right) \psi\right)_{x}, \omega_{x}^{2} \psi\right\rangle+\left\langle\bar{u}_{x x} \omega, \omega_{x} \psi\right\rangle \triangleq\left\langle D_{2}(\bar{\omega}), \omega_{x}^{2} \psi\right\rangle+\left\langle\bar{u}_{x x} \omega, \omega_{x} \psi\right\rangle .
\end{aligned}
$$

The first term in $I$ is a damping term. We plot the numerical values of $D_{2}(\bar{\omega})$ on the grid points in Figure 2. We can verify rigorously that it is bounded from above by -0.95 . Thus we have

$$
I=\left\langle D_{2}(\bar{\omega}), \omega_{x}^{2} \psi\right\rangle+\left\langle\bar{u}_{x x} \omega, \omega_{x} \psi\right\rangle \leq-0.95\left\langle\omega_{x}^{2}, \psi\right\rangle+\left\langle\bar{u}_{x x} \omega, \omega_{x} \psi\right\rangle \triangleq-0.95\left\langle\omega_{x}^{2}, \psi\right\rangle+I_{2},
$$


ON THE FINITE TIME BLOWUP OF THE DE GREGORIO MODEL FOR THE 3D EULER EQUATION 23

where $I_{2}=\left\langle\bar{u}_{x x} \omega, \omega_{x} \psi\right\rangle$. For $I I, I I I$, we note that

$$
\begin{aligned}
I I+I I I & =\left\langle u_{x x} \bar{\omega}+\left(u_{x}+c_{\omega}\right) \bar{\omega}_{x}-\left(u_{x}+c_{l}\right) \bar{\omega}_{x}-\left(c_{l} x+u\right) \bar{\omega}_{x x}, \omega_{x} \psi\right\rangle \\
& =\left\langle u_{x x} \bar{\omega}, \omega_{x} \psi\right\rangle-\left\langle\left(c_{l} x+u\right) \bar{\omega}_{x x}, \omega_{x} \psi\right\rangle \triangleq I I_{1}+I I_{2} .
\end{aligned}
$$

Using the definition of $\psi$, we get

$$
I I_{1}=\left\langle u_{x x} \bar{\omega}, \omega_{x} \psi\right\rangle=\left\langle u_{x x} \omega_{x},-\frac{1}{x}+\frac{x}{L^{2}}\right\rangle .
$$

Since $\omega_{x}(0)=0$ by the normalization condition and $u_{x x}(0)=0$ by the odd-even symmetry, we can use the same cancellation as we did in (2.23) to get

$$
\left\langle u_{x x} \omega_{x},-\frac{1}{x}\right\rangle=0, \quad\left\langle u_{x x} \omega_{x}, x\right\rangle=0 .
$$

Therefore $I I_{1}$ vanishes and we get

$$
I I+I I I=I I_{2}=-\left\langle\left(c_{l} x+u\right) \bar{\omega}_{x x}, \omega_{x} \psi\right\rangle,
$$

which is a cross term (in fact, it is a lower order term after integration by parts). The remaining linear terms in the weighted $H^{1}$ estimate are $I_{2}=\left\langle\bar{u}_{x x} \omega, \omega_{x} \psi\right\rangle$ in (3.38) and $I I_{2}$ in (3.39), which can be bounded by the interpolation between $\left\langle\omega^{2}, \varphi\right\rangle$ and $\left\langle\omega_{x}^{2}, \psi\right\rangle$. One can obtain a rough bound for these terms using the Cauchy-Schwartz inequality and the Hardy inequality

$$
\begin{aligned}
\left|I_{2}\right| & =\left|\left\langle\bar{u}_{x x} \omega, \omega_{x} \psi\right\rangle\right| \leq\left\langle\bar{u}_{x x}^{2}, \omega^{2} \psi\right\rangle^{1 / 2}\left\langle\omega_{x}^{2}, \psi\right\rangle^{1 / 2} \leq\left\|\bar{u}_{x x} \psi^{1 / 2} \varphi^{-1 / 2}\right\|_{L^{\infty}[0, L]}\left\langle\omega^{2}, \varphi\right\rangle^{1 / 2}\left\langle\omega_{x}^{2}, \psi\right\rangle^{1 / 2}, \\
\left|I I_{2}\right| & =\mid\left\langle\left(c_{l} x+u\right) \bar{\omega}_{x x}, \omega_{x} \psi \mid\right\rangle \leq\left\langle\left(c_{l} x+u\right)^{2}, x^{-2}+(x-L)^{-2}\right\rangle^{1 / 2}\left\langle\left(x^{-2}+(x-L)^{-2}\right)^{-1} \bar{\omega}_{x x}^{2} \psi, \omega_{x x} \psi\right\rangle^{1 / 2} \\
& \leq\left\{8\left(\left\langle g_{c_{\omega}}^{2}, \varphi^{-1}\right\rangle+\left\|\varphi^{-1}\right\|_{L^{\infty}[0, L]}\right) \cdot\left\|\left(x^{-2}+(x-L)^{-2}\right)^{-1} \bar{\omega}_{x x}^{2} \psi\right\|_{L^{\infty}[0, L]}\right\}^{1 / 2}\left\langle\omega^{2}, \varphi\right\rangle^{1 / 2}\left\langle\omega_{x}^{2}, \psi\right\rangle^{1 / 2},
\end{aligned}
$$

where $g_{c_{\omega}}$ is defined in (3.24). It follows

$$
\left|I_{2}\right|+\left|I I_{2}\right| \leq C_{1}(\bar{\omega})\left\langle\omega^{2}, \varphi\right\rangle^{1 / 2}\left\langle\omega_{x}^{2}, \psi\right\rangle^{1 / 2},
$$

where

$$
\begin{aligned}
C_{1}(\bar{\omega}) & \triangleq\left\|\bar{u}_{x x} \psi^{1 / 2} \varphi^{-1 / 2}\right\|_{L^{\infty}[0, L]} \\
& +\left\{8\left(\left\langle g_{c_{\omega}}^{2}, \varphi^{-1}\right\rangle+\left\|\varphi^{-1}\right\|_{L^{\infty}[0, L]}\right) \cdot\left\|\left(x^{-2}+(x-L)^{-2}\right)^{-1} \bar{\omega}_{x x}^{2} \psi\right\|_{L^{\infty}[0, L]}\right\}^{1 / 2} .
\end{aligned}
$$

From the definition of $\varphi, \psi(3.6),(3.35)$, the quantities appeared in $C_{1}(\bar{\omega})$ satisfy that

$$
\begin{aligned}
& \varphi^{-1}=O\left(\left(x^{-4}+(x-L)^{-2}\right)^{-1}\right),\left|\bar{u}_{x x} \psi^{1 / 2} \varphi^{-1 / 2}\right|=O\left(\bar{u}_{x x}\left(x^{-1}+(L-x)^{-1}\right)^{-1}\right), \\
& \left(x^{-2}+(x-L)^{-2}\right)^{-1} \bar{\omega}_{x x}^{2} \psi=O\left(\left(1+(x-L)^{-2}\right)^{-1} \bar{\omega}_{x x}^{2}\right) .
\end{aligned}
$$

In particular, these quantities are bounded for any $x \in[0, L]$ and thus $C_{1}(\bar{\omega})$ is finite.

Therefore, combining (3.36), (3.38), (3.39) and (3.40), we prove for any $\varepsilon>0$,

$$
\frac{1}{2} \frac{d}{d t}\left\langle\omega_{x}^{2}, \psi\right\rangle \leq-0.95\left\langle\omega_{x}^{2}, \psi\right\rangle+\varepsilon\left\langle\omega_{x}^{2}, \psi\right\rangle+(4 \varepsilon)^{-1} C_{1}(\bar{\omega})^{2}\left\langle\omega^{2}, \varphi\right\rangle+N_{2}+F_{2},
$$

From (3.34) and (3.42), we can choose $\varepsilon, \mu>0$ and construct the energy $E(t)^{2}=\left\langle\omega^{2}, \varphi\right\rangle+$ $\mu\left\langle\omega_{x}^{2}, \psi\right\rangle$ to close the weighted $L^{2}$ and weighted $H^{1}$ estimate at the linear level

$$
\frac{d}{d t} E(t)^{2} \leq-C(\mu, \varepsilon) E(t)^{2}+N_{1}+F_{1}+\mu\left(N_{2}+F_{2}\right),
$$

where $C(\mu, \varepsilon)>0$ depends on $\mu, \varepsilon$. For example, one can choose $\varepsilon=0.65, \mu=0.4 \varepsilon C_{1}(\bar{\omega})^{-2}$ to obtain $C(\mu, \varepsilon)=0.2$. 
3.3.1. Nonlinear stability. Recall that $N, F$ are defined in (3.5), $N_{1}, F_{1}$ in (3.7), and $N_{2}, F_{2}$ in (3.36).

Using integration by parts similar to that in (3.8) and (3.37), we obtain

$$
\begin{aligned}
& N_{1}+\mu N_{2}=\left\langle\frac{1}{2 \varphi}\left(\left(c_{l} x+u\right) \varphi\right)_{x}+\left(c_{\omega}+u_{x}\right), \omega^{2} \varphi\right\rangle+\mu\left\langle\frac{1}{2 \psi}\left(\left(c_{l} x+u\right) \psi\right)_{x}, \omega_{x}^{2} \psi\right\rangle . \\
= & \left\langle\frac{1}{2 \varphi}\left(c_{l} x+u\right) \varphi_{x}+\frac{3}{2}\left(c_{\omega}+u_{x}\right), \omega^{2} \varphi\right\rangle+\mu\left\langle\frac{1}{2 \psi}\left(c_{l} x+u\right) \psi_{x}+\frac{1}{2}\left(c_{\omega}+u_{x}\right), \omega_{x}^{2} \psi\right\rangle .
\end{aligned}
$$

Note that $\left|u_{x}\right|$ can be bounded by $E(t)$ as follows

$$
\begin{aligned}
\left\|u_{x}\right\|_{\infty} & \leq 2\left\|u_{x}\right\|_{L^{2}\left(\mathbb{R}^{+}\right)}^{1 / 2}\left\|u_{x x}\right\|_{L^{2}\left(\mathbb{R}^{+}\right)}^{1 / 2} \leq 2\|\omega\|_{2}^{1 / 2}\left\|\omega_{x}\right\|_{2}^{1 / 2} \leq\left. 2\left\|\varphi^{-1 / 2}\right\|\right|_{\infty} ^{1 / 2}\left\|\psi^{-1 / 2}\right\|_{\infty}^{1 / 2}\left\langle\omega^{2}, \varphi\right\rangle^{1 / 4}\left\langle\omega_{x}^{2}, \psi\right\rangle^{1 / 4} \\
& \leq 2 \mu^{-1 / 4}\left\|\varphi^{-1 / 2}\right\|_{\infty}^{1 / 2}\left\|\psi^{-1 / 2}\right\|_{\infty}^{1 / 2} E(t) \triangleq C_{2}(\bar{\omega}, \mu) E(t) .
\end{aligned}
$$

Recall $c_{l}=c_{\omega}$ (3.2). We have $c_{l} x+\left.u\right|_{x=0, L}=0$ and

$$
\left|c_{l} x+u\right| \leq \min (|x|,|L-x|) \cdot\left\|c_{\omega}+u_{x}\right\|_{L^{\infty}[0, L]} \leq 2 \min (|x|,|L-x|)|| u_{x} \|_{\infty} .
$$

Therefore, using the estimate of $u_{x}, c_{l} x+u$ and (3.44), we obtain

$$
\begin{aligned}
& N_{1}+\mu N_{2} \leq 2\left\{2+\left\|(|x| \wedge|L-x|)\left(\left|\frac{\varphi_{x}}{2 \varphi}\right|+\left|\frac{\psi_{x}}{2 \psi}\right|\right)\right\|_{\infty}\right\}\left\|u_{x}\right\|_{\infty}\left(\left\langle\omega^{2}, \varphi\right\rangle+\mu\left\langle\omega_{x}^{2}, \psi\right\rangle\right) \\
\leq & 2\left\{2+\left\|(|x| \wedge|L-x|)\left(\left|\frac{\varphi_{x}}{2 \varphi}\right|+\left|\frac{\psi_{x}}{2 \psi}\right|\right)\right\|_{\infty}\right\} C_{2}(\bar{\omega}, \mu) E(t)^{3} \triangleq C_{3}(\bar{\omega}, \mu) E(t)^{3} .
\end{aligned}
$$

To estimate the error term, we use the Cauchy-Schwartz inequality

$$
\begin{aligned}
F_{1}+\mu F_{2} & =\langle F(\bar{\omega}), \omega \varphi\rangle+\mu\left\langle F(\bar{\omega})_{x}, \omega_{x} \psi\right\rangle \\
& \leq\left(\left\langle F(\bar{\omega})^{2}, \varphi\right\rangle+\mu\left\langle F(\bar{\omega})_{x}^{2}, \psi\right\rangle\right)^{1 / 2} E(t) \triangleq \operatorname{error}(\bar{\omega}) E(t),
\end{aligned}
$$

Note that the above estimates except error $(\bar{\omega})$ are uniform for all approximate profiles sufficiently close to the self-similar solution. For example, for sufficiently small mesh size $h$, the difference between $\bar{\omega}_{h}$ and its limiting value as $h \rightarrow 0$ is of order $h$, so are the constants $C_{1}\left(\bar{\omega}_{h}\right)$ in (3.41) and $C_{3}\left(\bar{\omega}_{h}, \mu\right)$ in (3.45). Moreover, the approximate error error $(\bar{\omega})$ can be made arbitrarily small by choosing a very fine mesh size. In principle, by taking the numerical mesh size $h$ sufficiently small and combining (3.43), (3.45) and (3.46), we can complete the bootstrap argument. Then the remaining steps are the same as those in the proof of Theorem 1.2. Note that $C_{c}^{\infty}[-L, L]$ is dense in $L^{2}(\varphi) \cap H^{1}(\psi)$ with fixed $\omega_{x}(0)$. In fact, the weights near the corner $x= \pm L$ is of order $O\left((x-L)^{-2}\right)$ in the $L^{2}$ norm and $O(1)$ in the $H^{1}$ norm. Hence, we can choose an initial perturbation $\omega$ such that $\omega+\bar{\omega}$ is $C^{\infty}$ with compact support. The bootstrap and the convergence arguments similar to that in the proof of Theorem 1.2 imply that $\omega(t)+\bar{\omega}$, $c_{l}+\bar{c}_{l}=c_{\omega}(t)+\bar{c}_{\omega}$ remain close to $\bar{\omega}, \bar{c}_{\omega}<-0.69$ for all time $t>0$, respectively, and eventually converge to the self-similar profile $\omega_{\infty}$ with scaling $c_{l, \infty}=c_{\omega, \infty}<0$. After rescaling the self-similar profile using the argument similar to (2.40), we can obtain a self-similar profile with scaling $c_{l, \infty}=c_{\omega, \infty}=-1$. Thus we obtain a stable and expanding finite time self-similar blowup of the original De Gregorio model with blowup scaling $c_{l}=-1$.

In the supplementary material [2, we will provide much sharper estimates of the cross term (3.40), (3.42), the nonlinear term (3.45) and the error term (3.46). These sharper stability estimates and error estimate enable us to choose a modest mesh size to close the bootstrap argument. In particular, we choose $h=2.5 \cdot 10^{-5}$ and the computational cost of $\bar{\omega}_{h}$ is affordable even for a personal laptop computer. More specifically, we can prove the following refined estimate, which improves the estimate given by (3.42) significantly.

Lemma 3.1. The weighted $H^{1}$ estimate satisfies

$$
\frac{1}{2} \frac{d}{d t}\left\langle\omega_{x}^{2}, \psi\right\rangle=I+I I_{2}+N_{2}+F_{2} \leq-0.25\left\langle\omega_{x}^{2}, \psi\right\rangle+7.5\left\langle\omega^{2}, \varphi\right\rangle+N_{2}+F_{2},
$$

where $I, I I_{2}$ combine the damping and the cross terms and are defined in (3.38), (3.39), respectively. 
With this refined estimate and the refined estimate of nonlinear and error terms, we choose $\mu=0.02$ and bootstrap assumption $E(t)=\left\langle\omega^{2}, \varphi\right\rangle+\mu\left\langle\omega_{x}^{2}, \psi\right\rangle<5 \cdot 10^{-4}$ to complete the final bootstrap argument.

\section{Finite Time Blowup for $C^{\alpha}$ Initial Data}

In [10], Elgindi and Jeong obtained the $C^{\alpha}$ self-similar solution $\omega_{\alpha}$ of the Constantine-LaxMajda equation

$$
c_{l} x \omega_{x}=\left(c_{\omega}+u_{x}\right) w
$$

for all $\alpha \in(0,1]$, which reads

$$
\begin{aligned}
w_{\alpha} & =-\frac{2 \sin \left(\frac{\alpha \pi}{2}\right) \operatorname{sgn}(x)|x|^{\alpha}}{1+2 \cos \left(\frac{\alpha \pi}{2}\right)|x|^{\alpha}+|x|^{2 \alpha}}, \quad u_{\alpha, x}=\frac{2\left(1+\cos \left(\frac{\alpha \pi}{2}\right)|x|^{\alpha}\right)}{1+2 \cos \left(\frac{\alpha \pi}{2}\right)|x|^{\alpha}+|x|^{2 \alpha}}, \\
c_{l} & =\frac{1}{\alpha}, \quad c_{\omega}=-1,
\end{aligned}
$$

where $c_{l}, c_{\omega}$ are the scaling parameters. Based on these self-similar solutions, they further constructed the $C^{\alpha}$ self similar solution of the DG model for any $a$. However, such self-similar solution does not have finite energy in the sense that $\|u\|_{2}=+\infty$. In this section, we will show that the $C^{\alpha}$ self-similar solution of the CLM equation is stable under perturbation so that we can construct compactly supported initial data that develop self-similar blowup in finite time.

4.1. Stability of the $C^{\alpha}$ CLM Self-Similar Solution. In this Section, we prove the following result, which implies Theorem 1.3 directly.

Theorem 4.1. There exists some absolute constants $C_{1}, C_{2}, \mu$ with $C_{1} C_{2}<1 / 4$ and some weight functions $\varphi_{\alpha}, \psi_{\alpha}$, such that for any $\alpha \in(0,1 / 4]$ and $|a \alpha|<C_{1}$, if the initial data $\omega_{0}$ of the dynamical rescaling equation (2.3) satisfies

$$
E\left(\omega_{0}, \omega_{\alpha}\right)<C_{2}|a \alpha|
$$

where $E\left(\omega, \omega_{\alpha}\right)$ is given by

$$
E\left(w, w_{\alpha}\right)^{2} \triangleq\left\langle\left(\omega-\omega_{\alpha}\right)^{2}, \varphi_{\alpha}\right\rangle+\mu\left\langle\left(\omega_{x}-\omega_{\alpha, x}\right)^{2}, \psi_{\alpha}\right\rangle,
$$

then we have (a) the solution of (2.3) satisfies $E\left(\omega(t), \omega_{\alpha}\right)<C_{2}|a \alpha|$ for all $t \geq 0$; (b) the physical solution of the De Gregorio equation (1.3) with initial data $\omega_{0}$ develops a self-similar singularity in finite time.

Remark 4.2. (i) In the proof to be presented, we choose weights

$$
\varphi_{\alpha}=-\frac{1}{\operatorname{sgn}(x) \omega_{\alpha}} \frac{1+2 \cos \left(\frac{\alpha \pi}{2}\right)|x|^{\alpha}+|x|^{2 \alpha}}{|x|^{1+2 \alpha}}, \quad \psi_{\alpha}=\frac{1}{\alpha^{2}} \varphi_{\alpha} x^{2} .
$$

The reason we choose the above weight $\psi$ is that $\omega_{x}$ is about $\alpha \omega / x$. We choose these weights so that $\left\langle\omega^{2}, \varphi\right\rangle$ and $\left\langle\omega_{x}^{2}, \psi\right\rangle$ are comparable.

(ii) The $1 / 4$ upper bound on $\alpha$ is not a technical assumption but useful for simplifying the presentation. It will be clear in the proof that the results for $\alpha \in[1 / 4,1)$ can be obtained using a similar argument.

(iii) Throughout the proof, we impose $|a \alpha|<1$.

The proof of Theorem 4.1 is based on several uniform estimates on the self-similar solution and a bootstrap argument in the dynamical rescaling equation that is similar to the proof of small $|a|$ that we presented in Section 2 . 
4.1.1. Normalization Conditions and Approximate Steady State. The self-similar equation of DG model with parameter $a$ reads

$$
\left(c_{l} x+a u\right) \omega_{x}=\left(c_{\omega}+u_{x}\right) \omega .
$$

For any $a>0, \alpha \in(0,1)$, we construct $C^{\alpha}$ approximate self-similar profile of (4.5) below

$$
\omega_{\alpha}, \quad u_{\alpha}, \quad \bar{c}_{l, \alpha}=\frac{1}{\alpha}-a u_{\alpha, x}(0)=\frac{1}{\alpha}-2 a, \quad \bar{c}_{\omega}=-1 .
$$

The only difference between the above solution and the $C^{\alpha}$ self similar solutions of CLM (4.2) is the $c_{l}$ term. The above solution satisfies (4.5) up to an error

$$
F_{\alpha}\left(\omega_{\alpha}\right)=-\left(\bar{c}_{l} x-\frac{1}{\alpha} x+a u_{\alpha}\right) \omega_{\alpha, x}=-a\left(u_{\alpha}-u_{\alpha, x}(0) x\right) \omega_{\alpha, x} .
$$

Denote $\omega, u_{x}=H \omega$ as the perturbation around the approximate self-similar profile $\left(\omega_{\alpha}, u_{\alpha}\right)$. Also we make a change of variables $c_{l} \rightarrow \bar{c}_{l, \alpha}+c_{l}, c_{\omega} \rightarrow c_{\omega, \alpha}+c_{\omega}$ in (4.5) so that $c_{l}, c_{w}$ now denote the perturbations. Similar to the proof of Proposition 2.1, we consider the equation for $\omega$ and $u$

$$
\omega_{t}+\left(\bar{c}_{l, \alpha} x+a u_{\alpha}\right) \omega_{x}=\left(\bar{c}_{\omega}+u_{\alpha, x}\right) \omega+\left(u_{x}+c_{\omega}\right) \omega_{\alpha}-\left(a u+c_{l} x\right) \omega_{\alpha, x}+N(\omega)+F_{\alpha}\left(\omega_{\alpha}\right),
$$

where the error term $F_{\alpha}\left(\omega_{\alpha}\right)$ is given in (4.7) and the nonlinear part is given by

$$
N(\omega)=\left(c_{\omega}+u_{x}\right) \omega-\left(c_{l} x+a u\right) \omega_{x} .
$$

We choose the following normalization conditions for $c_{l}(t), c_{\omega}(t)$

$$
\begin{aligned}
& c_{l}(t)=-a u_{x}(t, 0), \quad c_{\omega}(t)=-u_{x}(t, 0) \\
\Longleftrightarrow & c_{l}(t)+\bar{c}_{l}=\frac{1}{\alpha}-a\left(u_{x}(t, 0)+u_{\alpha, x}(0)\right), \quad c_{\omega}+\bar{c}_{\omega}=1-\left(u_{x}(t, 0)+u_{\alpha, x}(0)\right),
\end{aligned}
$$

where we use $u_{\alpha, x}(0)=2$.

4.1.2. Estimate of the velocity, the self-similar solution and the error. We introduce the notation

$$
\tilde{u} \triangleq u-u_{x}(0) x, \quad \tilde{u}_{x}=u_{x}-u_{x}(0),
$$

and use the weighs defined in (4.4) to perform the $L^{2}, H^{1}$ estimates.

We first state some useful properties of the $C^{\alpha}$ approximate self-similar solution that we will use in our stability analysis.

Lemma 4.3. For $\alpha \in(0,1]$, we have the following estimates for the self-similar solutions defined in (4.2). (a) Uniform estimates on the damping effect

$$
\begin{aligned}
& \frac{1}{2 \varphi_{\alpha}}\left(\frac{1}{\alpha} x \varphi_{\alpha}\right)_{x}+\left(\bar{c}_{\omega}+u_{\alpha, x}\right)=-1 / 2, \\
& \frac{1}{2 \psi_{\alpha}}\left(\frac{1}{\alpha} x \psi_{\alpha}\right)_{x}+\left(\bar{c}_{\omega}+u_{\alpha, x}\right)-\frac{1}{\alpha}=-1 / 2, \\
& \frac{\left(u_{\alpha, x x} \psi_{\alpha}\right)_{x}}{2 \psi_{\alpha}} x^{2}=\frac{4 \alpha^{2}|x|^{\alpha}\left(|x|^{\alpha}+\cos \left(\frac{\alpha \pi}{2}\right)\right)}{\left(1+2 \cos \left(\frac{\alpha \pi}{2}\right)|x|^{\alpha}+|x|^{2 \alpha}\right)^{2}} \geq 0 .
\end{aligned}
$$

(b) Vorticity and velocity estimates:

$$
\begin{aligned}
& \left\|\frac{x w_{\alpha, x}}{w_{\alpha}}\right\|_{\infty} \lesssim \alpha, \quad\left\|\frac{x^{2} w_{\alpha, x x}}{w_{\alpha}}\right\|_{\infty} \lesssim \alpha, \quad\left\|\frac{x^{2} \omega_{\alpha, x x}+x \omega_{\alpha, x}}{\omega_{\alpha}}\right\|_{\infty} \lesssim \alpha^{2}, \\
& \left|\frac{u_{\alpha}}{x}-u_{\alpha, x}(0)\right| \lesssim|x|^{\alpha} \wedge 1, \quad\left|\frac{u_{\alpha}}{x}-u_{\alpha, x}\right| \lesssim \alpha\left(|x|^{\alpha} \wedge 1\right) .
\end{aligned}
$$


ON THE FINITE TIME BLOWUP OF THE DE GREGORIO MODEL FOR THE 3D EULER EQUATION 27

(c) Asymptotic estimates of $\varphi_{\alpha}, \psi_{\alpha}$ :

$$
\begin{aligned}
& \varphi_{\alpha} \asymp \frac{1}{\alpha}\left(|x|^{-1-3 \alpha}+|x|^{-1+\alpha}\right), \\
& \psi_{\alpha}=\frac{1}{\alpha^{2}} x^{2} \varphi_{\alpha} \asymp \frac{1}{\alpha^{3}}\left(|x|^{1-3 \alpha}+|x|^{1+\alpha}\right), \\
& \left\|\frac{x \psi_{\alpha, x}}{\psi_{\alpha}}-1\right\|_{\infty} \lesssim \alpha, \quad\left\|\frac{x \varphi_{\alpha, x}}{\varphi_{\alpha}}+1\right\|_{\infty} \lesssim \alpha,
\end{aligned}
$$

where $A \asymp B$ means that $A \leq C B$ and $B \leq C A$ for some universal constant $C$.

(d) The smallness of the weighted $L^{2}$ and $H^{1}$ errors:

$$
\begin{aligned}
& \left\langle F_{\alpha}\left(\omega_{\alpha}\right)^{2}, \varphi_{\alpha}\right\rangle \lesssim a^{2} \alpha^{2}, \quad\left\langle\left(F_{\alpha}\left(\omega_{\alpha}\right)\right)_{x}^{2}, \psi_{\alpha}\right\rangle \lesssim a^{2} \alpha^{2}, \\
& \left\langle\left(|x|^{\alpha} \wedge 1\right)^{2} \omega_{\alpha, x}^{2}, \psi_{\alpha}\right\rangle \lesssim 1 .
\end{aligned}
$$

These estimates are elementary and we defer the proof to the Appendix.

Remark 4.4. We will use (4.10) to show that the damping effect in the weighted $L^{2}$ and $H^{1}$ estimates is uniform for any $\alpha \in(0,1)$. (4.11) shows that we can gain a small factor $\alpha$ from $\omega_{\alpha, x}$. This enables us to show that the perturbation term $u \omega_{\alpha, x}$ is small. (4.13) shows that $x \psi_{\alpha, x} / \psi_{\alpha}, x \varphi_{\alpha, x} / \varphi_{\alpha}$ are close to 1 and -1 , respectively.

Lemma 4.5 ( $L^{\infty}$ estimate).

$$
\begin{aligned}
|| u_{x} \|_{\infty} & \lesssim\left\langle\omega^{2}, \varphi_{\alpha}\right\rangle^{1 / 4}\left\langle\omega_{x}^{2}, \psi_{\alpha}\right\rangle^{1 / 4} \\
\left|\tilde{u}_{x}-\frac{\tilde{u}}{x}\right| & \lesssim \alpha\left\langle\omega_{x}^{2}, \psi_{\alpha}\right\rangle^{1 / 2}\left|x^{\alpha}\right| \wedge 1 \lesssim \alpha\left\langle\omega_{x}^{2}, \psi_{\alpha}\right\rangle^{1 / 2}, \\
|\omega(x)| & \lesssim \alpha\left\langle\omega_{x}^{2}, \psi_{\alpha}\right\rangle^{1 / 2}\left|x^{\alpha}\right| \wedge 1
\end{aligned}
$$

where $\tilde{u}=u-u_{x}(0) x$.

We also defer the proof to the Appendix. (4.17) shows that we can gain a small factor $\alpha$ from $\tilde{u}_{x}-\frac{\tilde{u}}{x}=u_{x}-u / x$.

We use a strategy similar to that in the proof of Theorem 1.2 to prove Theorem 4.1. The key step is establishing linear stability by taking advantage of the following:

(a) the stretching effect $\bar{c}_{l, \alpha} x \omega_{x}$ and the damping term $\left(\bar{c}_{\omega}+u_{x, \alpha}\right) \omega$;

(b) the cancellation (A.12), A.6) involving the vortex stretching term $u_{x} \omega_{\alpha}$;

(c) the smallness of the convection term $a u \omega_{\alpha, x}$ (see (4.11) ) by choosing $|a \alpha|$ to be sufficiently small

To control the velocity $u$, we need to use Lemma A.4 in the Appendix, which states some nice properties of the Hilbert transform for a Hölder continuous function.

4.1.3. Linear Estimate. We first perform the weighted $L^{2}$ estimate with respect to (4.8). We proceed as follows

$$
\begin{aligned}
\frac{1}{2} \frac{d}{d t}\left\langle\omega^{2}, \varphi_{\alpha}\right\rangle & =\left\langle-\left(\bar{c}_{l, \alpha} x+a u_{\alpha}\right) \omega_{x}+\left(\bar{c}_{\omega}+u_{\alpha, x}\right) \omega, \omega \varphi_{\alpha}\right\rangle+\left\langle\left(u_{x}+c_{\omega}\right) \omega_{\alpha}, \omega \varphi_{\alpha}\right\rangle \\
& -\left\langle\left(a u+c_{l} x\right) \omega_{\alpha, x}, \omega \varphi_{\alpha}\right\rangle+\left\langle N(\omega), \omega \varphi_{\alpha}\right\rangle+\left\langle F_{\alpha}\left(\omega_{\alpha}\right), \omega \varphi_{\alpha}\right\rangle \\
& \triangleq I+I I+I I I+N+F .
\end{aligned}
$$

For $I$, we use integration by parts, (4.10) and $\bar{c}_{l, \alpha}=\frac{1}{\alpha}-a u_{\alpha, x}(0)$ to get

$$
\begin{aligned}
I & =\left\langle\frac{1}{2 \varphi_{\alpha}}\left(\left(\bar{c}_{l, \alpha} x+a u_{\alpha}\right) \varphi_{\alpha}\right)_{x}+\left(\bar{c}_{\omega}+u_{\alpha, x}\right), \omega^{2} \varphi_{\alpha}\right\rangle \\
& =-\frac{1}{2}\left\langle\omega^{2}, \varphi_{\alpha}\right\rangle+a\left\langle\frac{1}{2 \varphi_{\alpha}}\left(\left(u_{\alpha}-u_{\alpha, x}(0) x\right) \varphi_{\alpha}\right)_{x}, \omega^{2} \varphi_{\alpha}\right\rangle .
\end{aligned}
$$


For the second term, we use (4.12) and (4.13) to yield

$$
\begin{aligned}
& \left|\frac{1}{2 \varphi_{\alpha}}\left(\left(u_{\alpha}-u_{\alpha, x}(0) x\right) \varphi_{\alpha}\right)_{x}\right|=\left|\frac{1}{2}\left(u_{\alpha, x}-u_{\alpha, x}(0)\right)+\frac{u_{\alpha}-u_{\alpha, x}(0) x}{x} \frac{x \varphi_{\alpha, x}}{2 \varphi_{\alpha}}\right| \\
= & \left|\frac{1}{2}\left(u_{\alpha, x}-\frac{u_{\alpha}}{x}\right)+\frac{u_{\alpha}-u_{\alpha, x}(0) x}{x}\left(\frac{x \varphi_{\alpha, x}}{2 \varphi_{\alpha}}+\frac{1}{2}\right)\right| \lesssim \alpha+1 \cdot \alpha \lesssim \alpha .
\end{aligned}
$$

Combining (4.20) with the above estimate, we derive

$$
I \leq-\frac{1}{2}\left\langle\omega^{2}, \varphi_{\alpha}\right\rangle+C|a| \alpha\left\langle\omega^{2}, \varphi_{\alpha}\right\rangle=-\left(\frac{1}{2}-C|a| \alpha\right)\left\langle\omega^{2}, \varphi_{\alpha}\right\rangle,
$$

where $C>0$ is some universal constant.

Recall the definition of $\varphi_{\alpha}$ in (4.4). For $I I$, we use the cancellation (A.12) and (A.6) to get

$$
\begin{aligned}
I I & =\left\langle\tilde{u}_{x} \omega_{\alpha}, \omega \varphi_{\alpha}\right\rangle=-\left\langle\tilde{u}_{x} \omega \cdot \operatorname{sgn}(x),|x|^{-1-2 \alpha}+\left.2 \cos \left(\frac{\alpha \pi}{2}\right)|| x\right|^{-1-\alpha}+|x|^{-1}\right\rangle \\
& \leq-\left\langle\tilde{u}_{x} \omega \cdot \operatorname{sgn}(x),|x|^{-1}\right\rangle=-\frac{\pi}{2} u_{x}^{2}(0) \leq 0 .
\end{aligned}
$$

For $I I I$, we have

$$
\begin{aligned}
|I I I| & =\left|\left\langle\left(a u+c_{l} x\right) \omega_{\alpha, x}, \omega \varphi_{\alpha}\right\rangle\right|=\left|a\left\langle\frac{\tilde{u}}{x} \frac{\omega_{\alpha, x} x}{\omega_{\alpha}}, \omega \frac{1+2 \cos \left(\frac{\alpha \pi}{2}\right)|x|^{\alpha}+|x|^{2 \alpha}}{|x|^{1+2 \alpha}}\right\rangle\right| \\
& \lesssim|a|\left\langle\left|\frac{\tilde{u}}{x}\right|\left|\frac{\omega_{\alpha, x} x}{\omega_{\alpha}}\right|,|\omega|\left(|x|^{-1-2 \alpha}+|x|^{-1}\right)\right\rangle .
\end{aligned}
$$

Using the estimate for $\omega_{\alpha}$ (4.11) and the Hardy inequality (A.13), we obtain

$$
\begin{aligned}
|I I I| & \lesssim|a| \alpha\left\langle\left|\frac{\tilde{u}}{x}\right|,|\omega|\left(|x|^{-1-2 \alpha}+|x|^{-1}\right)\right\rangle \\
& \lesssim|a| \alpha\left\langle\tilde{u}^{2},|x|^{-3-3 \alpha}\right\rangle^{1 / 2}\left\langle\omega^{2},|x|^{-1-\alpha}\right\rangle^{1 / 2}+|a| \alpha\left\langle\tilde{u}^{2},|x|^{-3-\alpha}\right\rangle^{1 / 2}\left\langle\omega^{2},|x|^{-1+\alpha}\right\rangle^{1 / 2} \\
& \lesssim|a| \alpha \alpha^{-1}\left\langle\omega^{2},|x|^{-1-3 \alpha}\right\rangle^{1 / 2}\left\langle\omega^{2},|x|^{-1-\alpha}\right\rangle^{1 / 2}+|a| \alpha \alpha^{-1}\left\langle\omega^{2},|x|^{-1-\alpha}\right\rangle^{1 / 2}\left\langle\omega^{2},|x|^{-1+\alpha}\right\rangle^{1 / 2} \\
& \lesssim|a| \alpha\left\langle\omega^{2}, \varphi_{\alpha}\right\rangle,
\end{aligned}
$$

where we have used (4.13) to obtain the last inequality.

Plugging (4.21), (4.22) and (4.23) in (4.19), we arrive at

$$
\frac{1}{2} \frac{d}{d t}\left\langle\omega^{2}, \varphi_{\alpha}\right\rangle \leq-\left(\frac{1}{2}-C|a| \alpha\right)\left\langle\omega^{2}, \varphi_{\alpha}\right\rangle+\left\langle N(\omega), \omega \varphi_{\alpha}\right\rangle+\left\langle F_{\alpha}\left(\omega_{\alpha}\right), \omega \varphi_{\alpha}\right\rangle .
$$

4.1.4. Weighted $H^{1}$ Estimate. Recall the definition of the weight $\psi_{\alpha}$ in (4.4). We now perform the weighted $H^{1}$ estimate with respect to (4.8)

$$
\begin{aligned}
\frac{1}{2} \frac{d}{d t}\left\langle\omega_{x}^{2}, \psi_{\alpha}\right\rangle & =\left\langle-\left(\left(\bar{c}_{l, \alpha} x+a u_{\alpha}\right) \omega_{x}\right)_{x}+\left(\left(\bar{c}_{\omega}+u_{\alpha, x}\right) \omega\right)_{x}, \omega_{x} \psi_{\alpha}\right\rangle+\left\langle\left(\left(u_{x}+c_{\omega}\right) \omega_{\alpha}\right)_{x}, \omega_{x} \psi_{\alpha}\right\rangle \\
& -\left\langle\left(\left(a u+c_{l} x\right) \omega_{\alpha, x}\right)_{x}, \omega_{x} \psi_{\alpha}\right\rangle+\left\langle N(\omega)_{x} \cdot \omega_{x} \psi_{\alpha}\right\rangle+\left\langle F_{\alpha}\left(\omega_{\alpha}\right)_{x}, \omega_{x} \psi_{\alpha}\right\rangle \\
& \triangleq I+I I+I I I+N_{2}+F_{2} .
\end{aligned}
$$

For $I$, we again use integration by parts to obtain

$$
\begin{aligned}
I & =\left\langle-\left(\bar{c}_{l, \alpha} x+a u_{\alpha}\right) \omega_{x x}-\left(\bar{c}_{l, \alpha}+a u_{\alpha, x}\right) \omega_{x}+\left(\bar{c}_{\omega}+u_{\alpha, x}\right) \omega_{x}, \omega_{x} \psi_{\alpha}\right\rangle+\left\langle u_{\alpha, x x} \omega, \omega_{x} \psi\right\rangle \\
& =\left\langle\frac{\left(\left(\bar{c}_{l, \alpha} x+a u_{\alpha}\right) \psi_{\alpha}\right)_{x}}{2 \psi_{\alpha}}-\left(\bar{c}_{l, \alpha}+a u_{\alpha, x}\right)+\left(\bar{c}_{\omega}+u_{\alpha, x}\right), \omega_{x}^{2} \psi_{\alpha}\right\rangle-\left\langle\frac{\left(u_{\alpha, x x} \psi_{\alpha}\right)_{x}}{2 \psi_{\alpha}}, \omega^{2} \psi\right\rangle \triangleq I_{1}+I_{2} .
\end{aligned}
$$

From (4.10), we get

$$
I_{2} \leq 0
$$

Recall that $\bar{c}_{l, \alpha}=\frac{1}{\alpha}-a u_{\alpha, x}$ and

$$
\bar{c}_{l, \alpha} x+a u_{\alpha, x}=\frac{1}{\alpha}+a\left(u_{\alpha}-u_{\alpha, x} x\right)=\frac{1}{\alpha}+a \tilde{u}_{\alpha}, \quad \bar{c}_{l, \alpha}+a u_{\alpha ; x}=\frac{1}{\alpha}+\tilde{u}_{\alpha, x} .
$$


ON THE FINITE TIME BLOWUP OF THE DE GREGORIO MODEL FOR THE 3D EULER EQUATION 29

We can use (4.10) to obtain

$$
\begin{aligned}
I_{1} & =-\frac{1}{2}\left\langle\omega_{x}^{2}, \psi_{\alpha}\right\rangle+a\left\langle\frac{\left(\tilde{u}_{\alpha} \psi_{\alpha}\right)_{x}}{2 \psi_{\alpha}}-\tilde{u}_{\alpha, x}, \omega_{x}^{2} \psi_{\alpha}\right\rangle \\
& =-\frac{1}{2}\left\langle\omega_{x}^{2}, \psi_{\alpha}\right\rangle+a\left\langle\frac{\tilde{u}_{\alpha}}{x} \frac{x \psi_{\alpha, x}}{2 \psi_{\alpha}}-\frac{\tilde{u}_{\alpha, x}}{2},, \omega_{x}^{2} \psi_{\alpha}\right\rangle .
\end{aligned}
$$

Using (4.12) and (4.13), we get

$$
\left|\frac{\tilde{u}_{\alpha}}{x} \frac{x \psi_{\alpha, x}}{2 \psi_{\alpha}}-\frac{\tilde{u}_{\alpha, x}}{2}\right| \leq\left|\frac{\tilde{u}_{\alpha}}{x}\right| \cdot\left|\frac{x \psi_{\alpha, x}}{2 \psi_{\alpha}}-\frac{1}{2}\right|+\frac{1}{2}\left|\frac{\tilde{u}_{\alpha}}{x}-\tilde{u}_{\alpha, x}\right| \lesssim|| u_{\alpha, x} \|_{\infty} \alpha+\alpha \lesssim \alpha .
$$

It follows that

$$
\begin{aligned}
& I_{1} \leq-\frac{1}{2}\left\langle\omega_{x}^{2}, \psi_{\alpha}\right\rangle+C|a| \alpha\left\langle\omega_{x}^{2}, \psi_{\alpha}\right\rangle=-\left(\frac{1}{2}-C|a| \alpha\right)\left\langle\omega_{x}^{2}, \psi_{\alpha}\right\rangle, \\
\Rightarrow & I=I_{1}+I_{2} \leq I_{1} \leq-\left(\frac{1}{2}-C|a| \alpha\right)\left\langle\omega_{x}^{2}, \psi_{\alpha}\right\rangle .
\end{aligned}
$$

For $I I$, we have

$$
I I=-\left\langle u_{x x} \omega_{\alpha}, \omega_{x} \psi_{\alpha}\right\rangle+\left\langle\tilde{u}_{x} \omega_{\alpha, x}, \omega_{x} \psi_{\alpha}\right\rangle \triangleq I I_{1}+I I_{2} .
$$

Note that

$$
H\left(\omega_{x}\right)=u_{x x}, H\left(\omega_{x} x\right)(0)=0 \Rightarrow H\left(\omega_{x} x\right)=u_{x x} x
$$

Moreover, we have that $\omega_{x} x$ is odd, $\left(x u_{x x}\right)(0)=\left(x \omega_{x}\right)(0)=0$. Applying the cancellation A.12), (A.6) with $\left(u_{x}, \omega\right)$ replaced by $\left(x u_{x x}, x \omega_{x}\right)$, we can estimate $I I_{1}$ as follows

$$
\begin{aligned}
I I_{1} & =-\left\langle\left(x u_{x x}\right)\left(x \omega_{x}\right), \frac{1}{\alpha^{2}} \frac{\left(1+2 \cos \left(\frac{\alpha \pi}{2}\right)|x|^{\alpha}+|x|^{2 \alpha}\right)}{\operatorname{sgn}(x)|x|^{1+2 \alpha}}\right\rangle \\
& \leq-\left\langle\left(x u_{x x}\right)\left(x \omega_{x}\right), \frac{1}{\operatorname{sgn}(x) \alpha^{2}|x|}\right\rangle=-\frac{\pi}{2 \alpha^{2}}\left(x u_{x x}\right)(0)^{2}=0 .
\end{aligned}
$$

The remaining terms in the weighted $H^{1}$ estimate are $I I_{2}$ in (4.27) and $I I I$ in (4.25), which can be decomposed as follows

$$
\begin{aligned}
I I I & =-\left\langle\left(\left(a u+c_{l} x\right) \omega_{\alpha, x}\right)_{x}, \omega_{x} \psi_{\alpha}\right\rangle=-a\left\langle\tilde{u}_{x} \omega_{\alpha, x}+\tilde{u} \omega_{\alpha, x x}, \omega_{x} \psi_{\alpha}\right\rangle \\
& =-a\left\langle\left(\tilde{u}_{x}-\frac{\tilde{u}}{x}\right) \omega_{\alpha, x}, \omega_{x} \psi_{\alpha}\right\rangle-a\left\langle\frac{\tilde{u}}{x}\left(\omega_{\alpha, x}+x \omega_{\alpha, x x}\right), \omega_{x} \psi_{\alpha}\right\rangle \triangleq I I I_{1}+I I I_{2} .
\end{aligned}
$$

We perform the estimate of $I I I_{1}, I I I_{2}$ and the estimate of $I I_{2}$ can be done similarly. Using the pointwise estimate (4.17) and the Cauchy Schwartz inequality, we can estimate $I I I_{1}$ as follows

$$
\begin{aligned}
I I I_{1} & \leq|a| \alpha\left\langle\omega_{x}^{2}, \psi_{\alpha}\right\rangle^{1 / 2} \cdot\left\langle\left(|x|^{\alpha} \wedge 1\right)\left|\omega_{\alpha, x}\right|,\left|\omega_{x}\right| \psi_{\alpha}\right\rangle \\
& \lesssim|a| \alpha\left\langle\omega_{x}^{2}, \psi_{\alpha}\right\rangle \cdot\left\langle\left(|x|^{\alpha} \wedge 1\right)^{2} \omega_{\alpha, x}^{2}, \psi_{\alpha}\right\rangle^{1 / 2} \lesssim|a| \alpha\left\langle\omega_{x}^{2}, \psi_{\alpha}\right\rangle,
\end{aligned}
$$

where we have used (4.15) to obtain the last inequality.

For $I I I_{2}$, we first use (4.11) to obtain

$$
\begin{aligned}
\left|I I I_{2}\right| & =|a|\left|\left\langle\frac{\tilde{u}}{x}\left(\omega_{\alpha, x}+x \omega_{\alpha, x x}\right), \omega_{x}|x|^{1-2 \alpha} \frac{\left(1+2 \cos \left(\frac{\alpha \pi}{2}\right)|x|^{\alpha}+|x|^{2 \alpha}\right)}{\alpha^{2} \operatorname{sgn}(x) \omega_{\alpha}}\right\rangle\right| \\
& =|a|\left|\left\langle\frac{\tilde{u}}{x^{2}} \frac{x\left(\omega_{\alpha, x}+x \omega_{\alpha, x x}\right)}{\operatorname{sgn}(x) \omega_{\alpha}}, \omega_{x}|x|^{1-2 \alpha} \frac{\left(1+2 \cos \left(\frac{\alpha \pi}{2}\right)|x|^{\alpha}+|x|^{2 \alpha}\right)}{\alpha^{2}}\right\rangle\right| \\
& \lesssim|a|\left\langle\left|\frac{\tilde{u}}{x^{2}}\right| \alpha^{2},\left|\omega_{x}\right||x|^{1-2 \alpha} \frac{\left(1+2 \cos \left(\frac{\alpha \pi}{2}\right)|x|^{\alpha}+|x|^{2 \alpha}\right)}{\alpha^{2}}\right\rangle \\
& =|a|\left\langle\left|\frac{\tilde{u}}{x}\right|,\left|\omega_{x}\right|\left(1+2 \cos \left(\frac{\alpha \pi}{2}\right)|x|^{-\alpha}+|x|^{-2 \alpha}\right)\right\rangle \lesssim|a|\left\langle\left|\frac{\tilde{u}}{x}\right|,\left|\omega_{x}\right|\left(1+|x|^{-2 \alpha}\right)\right\rangle .
\end{aligned}
$$


Then we use the Hardy inequality (A.13) to estimate $\tilde{u}$

$$
\begin{aligned}
\left|I I I_{2}\right| & \lesssim|a|\left\langle\left|\frac{\tilde{u}}{x}\right|,\left|\omega_{x}\right|\left(1+|x|^{-2 \alpha}\right)\right\rangle \\
& \lesssim|a|\left\langle\tilde{u}^{2},|x|^{-3-3 \alpha}\right\rangle^{1 / 2}\left\langle\omega_{x}^{2},|x|^{1-\alpha}\right\rangle^{1 / 2}+|a|\left\langle\tilde{u}^{2},|x|^{-3-\alpha}\right\rangle^{1 / 2}\left\langle\omega_{x}^{2},|x|^{1+\alpha}\right\rangle^{1 / 2} \\
& \lesssim|a| \alpha^{-1}\left\langle\omega^{2},|x|^{-1-3 \alpha}\right\rangle^{1 / 2}\left\langle\omega_{x}^{2},|x|^{1-\alpha}\right\rangle^{1 / 2}+|a| \alpha^{-1}\left\langle\omega^{2},|x|^{-1-\alpha}\right\rangle^{1 / 2}\left\langle\omega_{x}^{2},|x|^{1+\alpha}\right\rangle^{1 / 2} .
\end{aligned}
$$

Using (4.13), we derive

$$
\left|I I I_{2}\right| \lesssim|a| \alpha^{-1} \alpha^{1 / 2}\left\langle\omega^{2}, \varphi_{\alpha}\right\rangle^{1 / 2} \alpha^{3 / 2}\left\langle\omega_{x}^{2}, \psi_{\alpha}\right\rangle^{1 / 2}=|a| \alpha\left\langle\omega^{2}, \varphi_{\alpha}\right\rangle^{1 / 2}\left\langle\omega_{x}^{2}, \psi_{\alpha}\right\rangle^{1 / 2} .
$$

Similarly, for $I I_{2}$, using the smallness $\left\|\frac{x w_{\alpha, x}}{w_{\alpha}}\right\|_{\infty} \lesssim \alpha$ in (4.11), the weighted estimate (A.10) and estimate of $\psi_{\alpha}, \varphi_{\alpha}$ (4.13), one can obtain

$$
\left|I I_{2}\right| \lesssim\left\langle\omega^{2}, \varphi_{\alpha}\right\rangle^{1 / 2}\left\langle\omega_{x}^{2}, \psi_{\alpha}\right\rangle^{1 / 2}
$$

Plugging (4.26), (4.27), (4.28), (4.29), (4.30) and (4.31) in (4.25), we obtain

$$
\begin{aligned}
\frac{1}{2} \frac{d}{d t}\left\langle\omega_{x}^{2}, \psi_{\alpha}\right\rangle & \leq-\left(\frac{1}{2}-C|a| \alpha\right)\left\langle\omega_{x}^{2}, \psi_{\alpha}\right\rangle+C\left\langle\omega^{2}, \varphi_{\alpha}\right\rangle^{1 / 2}\left\langle\omega_{x}^{2}, \psi_{\alpha}\right\rangle^{1 / 2} \\
& +\left\langle N(\omega)_{x}, \omega_{x} \psi_{\alpha}\right\rangle+\left\langle F_{\alpha}\left(\omega_{\alpha}\right)_{x}, \omega_{x} \psi_{\alpha}\right\rangle,
\end{aligned}
$$

for some universal constant $C$, where we have used $|a \alpha|<1$.

In the following two subsections, we aim to control the nonlinear and error terms

$$
\left\langle N(\omega), \omega \varphi_{\alpha}\right\rangle,\left\langle F_{\alpha}\left(\omega_{\alpha}\right), \omega \varphi_{\alpha}\right\rangle,\left\langle N(\omega)_{x}, \omega_{x} \psi_{\alpha}\right\rangle,\left\langle F_{\alpha}\left(\omega_{\alpha}\right)_{x}, \omega_{x} \psi_{\alpha}\right\rangle
$$

in (4.24) and (4.32).

\subsubsection{Estimates of nonlinear terms. Recall that}

$$
c_{l} x+a u=a\left(u-u_{x}(0) x\right)=a \tilde{u}, \quad c_{\omega}+u_{x}=u_{x}-u_{x}(0)=\tilde{u}_{x} .
$$

For the nonlinear terms in (4.24) and (4.32), we use integration by parts to obtain

$$
\begin{aligned}
\left\langle N(\omega), \omega \varphi_{\alpha}\right\rangle & =\left\langle\left(c_{\omega}+u_{x}\right) \omega-\left(c_{l} x+a u\right) \omega_{x}, \omega \varphi_{\alpha}\right\rangle=\left\langle\tilde{u}_{x}+\frac{\left(a \tilde{u} \varphi_{\alpha}\right)_{x}}{2 \varphi_{\alpha}}, \omega^{2} \varphi_{\alpha}\right\rangle \\
& =\left\langle\tilde{u}_{x}, \omega^{2} \varphi_{\alpha}\right\rangle+\frac{a}{2}\left\langle\left(\tilde{u}_{x}+\frac{\tilde{u}}{x} \frac{x \varphi_{\alpha, x}}{\varphi_{\alpha}}\right), \omega^{2} \varphi_{\alpha}\right\rangle \triangleq I_{1}+I_{2}, \\
\left\langle N(\omega)_{x}, \omega_{x} \psi_{\alpha}\right\rangle & =\left\langle\left(\left(c_{\omega}+u_{x}\right) \omega-\left(c_{l} x+a u\right) \omega_{x}\right)_{x}, \omega_{x} \psi_{\alpha}\right\rangle \\
& =\left\langle u_{x x} \omega+\tilde{u}_{x} \omega_{x}, \omega_{x} \psi_{\alpha}\right\rangle-a\left\langle\tilde{u}_{x} \omega_{x}+\tilde{u} \omega_{x x}, \omega_{x} \psi_{\alpha}\right\rangle \\
& =\left\langle\tilde{u}_{x} \omega_{x}, \omega_{x} \psi_{\alpha}\right\rangle+\left\langle u_{x x} \omega, \omega_{x} \psi_{\alpha}\right\rangle+a\left\langle-\tilde{u}_{x}+\frac{\left(\tilde{u} \psi_{\alpha}\right)_{x}}{2 \psi_{\alpha}}, \omega_{x}^{2} \psi_{\alpha}\right\rangle \\
& \triangleq I I_{1}+I I_{2}+I I_{3} .
\end{aligned}
$$

For each term $I_{i}, I I_{j}$, we use $L^{\infty}+L^{2}+L^{2}$ type of estimates. We use Lemma 4.5 to control the $L^{\infty}$ norm of $\omega, \tilde{u} / x, \tilde{u}_{x}$ or $\tilde{u}_{x}-\tilde{u} / x$, and use $\left\langle\omega^{2}, \varphi_{\alpha}\right\rangle,\left\langle\omega_{x}^{2}, \psi_{\alpha}\right\rangle$ to control other terms. We present the estimate of $I I_{3}$ that has a large coefficient $a$ and is more complicated and other terms can be done similarly. For $I I_{3}$, we notice that

$$
-\tilde{u}_{x}+\frac{\left(\tilde{u} \psi_{\alpha}\right)_{x}}{2 \psi_{\alpha}}=-\frac{1}{2} \tilde{u}_{x}+\frac{1}{2} \frac{\tilde{u}}{x} \frac{\psi_{\alpha, x} x}{\psi_{\alpha}}=-\frac{1}{2}\left(\tilde{u}_{x}-\frac{\tilde{u}}{x}\right)+\frac{1}{2} \frac{\tilde{u}}{x}\left(\frac{\psi_{\alpha, x} x}{\psi_{\alpha}}-1\right) .
$$

Then we use the $L^{\infty}$ estimate (4.17) to control $\tilde{u}_{x}-\tilde{u} / x$, (4.16) to control $\tilde{u} / x=u / x-u_{x}(0)$ and (4.13) to estimate the terms involving $\psi_{\alpha}$. This gives

$$
\begin{aligned}
& I I_{3}=\frac{a}{2}\left\langle-\left(\tilde{u}_{x}-\frac{\tilde{u}}{x}\right)+\frac{\tilde{u}}{x}\left(\frac{\psi_{\alpha, x} x}{\psi_{\alpha}}-1\right), \omega_{x}^{2} \psi_{\alpha}\right\rangle \\
& \lesssim|a|\left(\left\|\tilde{u}_{x}-\left.\frac{\tilde{u}}{x}\right|_{L^{\infty}}+\right\| u_{x}\left\|_{\infty}\right\| \frac{\psi_{\alpha, x} x}{\psi_{\alpha}}-1 \|_{L^{\infty}}\right)\left\langle\omega_{x}^{2}, \psi_{\alpha}\right\rangle \\
& \lesssim\left(|a| \alpha\left\langle\omega_{x}^{2}, \psi_{\alpha}\right\rangle^{1 / 2}+|a| \alpha\left\langle\omega^{2}, \varphi_{\alpha}\right\rangle^{1 / 4}\left\langle\omega_{x}^{2}, \psi_{\alpha}\right\rangle^{1 / 4}\right)\left\langle\omega_{x}^{2}, \psi_{\alpha}\right\rangle \lesssim\left(\left\langle\omega^{2}, \varphi_{\alpha}\right\rangle+\left\langle\omega_{x}^{2}, \psi_{\alpha}\right\rangle\right)^{3 / 2},
\end{aligned}
$$


ON THE FINITE TIME BLOWUP OF THE DE GREGORIO MODEL FOR THE 3D EULER EQUATION 31

where we have used $|a \alpha|<1$. Similarly, we have

$$
I_{1}, I_{2}, I I_{1}, I I_{2} \lesssim\left(\left\langle\omega^{2}, \varphi_{\alpha}\right\rangle+\left\langle\omega_{x}^{2}, \psi_{\alpha}\right\rangle\right)^{3 / 2} .
$$

Combining (4.33) and (4.34), we obtain the following estimates for the nonlinear terms

$$
\begin{aligned}
\left\langle N(\omega), \omega \varphi_{\alpha}\right\rangle & =I_{1}+I_{2} \lesssim\left(\left\langle\omega^{2}, \varphi_{\alpha}\right\rangle+\left\langle\omega_{x}^{2}, \psi_{\alpha}\right\rangle\right)^{3 / 2}, \\
\left\langle N(\omega)_{x}, \omega_{x} \psi_{\alpha}\right\rangle & =I I_{1}+I I_{2}+I I_{3} \lesssim\left(\left\langle\omega^{2}, \varphi_{\alpha}\right\rangle+\left\langle\omega_{x}^{2}, \psi_{\alpha}\right\rangle\right)^{3 / 2} .
\end{aligned}
$$

4.1.6. Estimates of the error terms. Recall the error terms in the weighted $L^{2}, H^{1}$ estimates in (4.24) and (4.32) are given by

$$
\left\langle F_{\alpha}\left(\omega_{\alpha}\right), \omega \varphi_{\alpha}\right\rangle, \quad\left\langle\left(F_{\alpha}\left(\omega_{\alpha}\right)\right)_{x}, \omega_{x} \psi_{\alpha}\right\rangle
$$

Using the Cauchy-Schwartz inequality and the error estimate (4.14), we obtain

$$
\begin{aligned}
\left\langle F_{\alpha}\left(\omega_{\alpha}\right), \omega \varphi_{\alpha}\right\rangle & \leq\left\langle F_{\alpha}\left(\omega_{\alpha}\right)^{2}, \varphi_{\alpha}\right\rangle^{1 / 2}\left\langle\omega^{2}, \varphi_{\alpha}\right\rangle^{1 / 2} \lesssim|a| \alpha\left\langle\omega^{2}, \varphi_{\alpha}\right\rangle^{1 / 2} \\
\left\langle\left(F_{\alpha}\left(\omega_{\alpha}\right)\right)_{x}, \omega_{x} \psi_{\alpha}\right\rangle & \leq\left\langle\left(F_{\alpha}\left(\omega_{\alpha}\right)\right)_{x}^{2}, \psi_{\alpha}\right\rangle^{1 / 2}\left\langle\omega_{x}^{2}, \psi_{\alpha}\right\rangle^{1 / 2} \lesssim|a| \alpha\left\langle\omega_{x}^{2}, \psi_{\alpha}\right\rangle^{1 / 2} .
\end{aligned}
$$

4.1.7. Nonlinear stability. Let $0<\mu<1$ be some parameter to be determined and we introduce the following energy

$$
E^{2}(t) \triangleq\left\langle\omega^{2}, \varphi_{\alpha}\right\rangle+\mu\left\langle\omega_{x}^{2}, \psi_{\alpha}\right\rangle .
$$

Using the weighted $L^{2}, H^{1}$ estimates (4.24), (4.32), the estimates of nonlinear term (4.35) and the estimates of error term (4.36), we obtain ( (4.24) $+\mu$ (4.32)

$$
\begin{aligned}
& \frac{1}{2} \frac{d}{d t}\left(\left\langle\omega^{2}, \varphi_{\alpha}\right\rangle+\mu\left\langle\omega_{x}^{2}, \psi_{\alpha}\right\rangle\right) \\
\leq & \underbrace{\left(\frac{1}{2}-C|a| \alpha\right)\left(\left\langle\omega^{2}, \varphi_{\alpha}\right\rangle+\mu\left\langle\omega_{x}^{2}, \psi_{\alpha}\right\rangle\right)+\mu C\left\langle\omega^{2}, \varphi_{\alpha}\right\rangle^{1 / 2}\left\langle\omega_{x}^{2}, \psi_{\alpha}\right\rangle^{1 / 2}}_{Q} \\
& +\underbrace{C|a| \alpha\left(\left\langle\omega^{2}, \varphi_{\alpha}\right\rangle^{1 / 2}+\mu\left\langle\omega_{x}^{2}, \psi_{\alpha}\right\rangle^{1 / 2}\right)}_{\text {error term }}+\underbrace{C(\mu)\left(\left\langle\omega^{2}, \varphi_{\alpha}\right\rangle+\mu\left\langle\omega_{x}^{2}, \psi_{\alpha}\right\rangle\right)^{3 / 2}}_{\text {nonlinear }},
\end{aligned}
$$

where $C$ is some universal constant, $C(\mu)$ depends on $\mu$ and we have used

$$
(1+\mu)\left(\left\langle\omega^{2}, \varphi_{\alpha}\right\rangle+\left\langle\omega_{x}^{2}, \psi_{\alpha}\right\rangle\right)^{3 / 2} \leq C(\mu)\left(\left\langle\omega^{2}, \varphi_{\alpha}\right\rangle+\mu\left\langle\omega_{x}^{2}, \psi_{\alpha}\right\rangle\right)^{3 / 2},
$$

for the nonlinear term in (4.35). Next, we argue that if $|a \alpha|<1$ and $\mu$ is small, the quadratic part $Q$ is negative, i.e. we obtain inviscid damping in the energy norm, $E$. First, we choose an absolute constant $\mu \in(0,1)$ such that

$$
C \sqrt{\mu}<\frac{1}{4}
$$

We fix $\mu$ from now on. Applying the inequality $\sqrt{\mu} a b \leq\left(a^{2}+\mu b^{2}\right) / 2$, we obtain

$$
\mu C\left\langle\omega^{2}, \varphi_{\alpha}\right\rangle^{1 / 2}\left\langle\omega_{x}^{2}, \psi_{\alpha}\right\rangle^{1 / 2} \leq \frac{1}{2} C \sqrt{\mu}\left(\left\langle\omega^{2}, \varphi_{\alpha}\right\rangle+\mu\left\langle\omega_{x}^{2}, \psi_{\alpha}\right\rangle\right)<\frac{1}{8}\left(\left\langle\omega^{2}, \varphi_{\alpha}\right\rangle+\mu\left\langle\omega_{x}^{2}, \psi_{\alpha}\right\rangle\right) .
$$

Using the above inequality, we can bound the quadratic term in (4.37) as follows:

$$
\begin{aligned}
Q & \leq-\left(\frac{1}{2}-C|a| \alpha\right)\left(\left\langle\omega^{2}, \varphi_{\alpha}\right\rangle+\mu\left\langle\omega_{x}^{2}, \psi_{\alpha}\right\rangle\right)+\frac{1}{8}\left(\left\langle\omega^{2}, \varphi_{\alpha}\right\rangle+\mu\left\langle\omega_{x}^{2}, \psi_{\alpha}\right\rangle\right) \\
& =-\left(\frac{3}{8}-C|a| \alpha\right)\left(\left\langle\omega^{2}, \varphi_{\alpha}\right\rangle+\mu\left\langle\omega_{x}^{2}, \psi_{\alpha}\right\rangle\right)=-\left(\frac{3}{8}-C|a| \alpha\right) E^{2}(t) .
\end{aligned}
$$

Since $\mu$ is an absolute constant, so is the constant $C(\mu)$ in (4.37). We can further simplify the error term and nonlinear term in (4.37) to obtain

$$
\frac{1}{2} \frac{d}{d t} E^{2}(t) \leq-\left(\frac{3}{8}-C|a| \alpha\right) E^{2}(t)+C|a| \alpha E(t)+C E^{3}(t),
$$

where the constant $C>1$ is an absolute constant. 
From (4.13), we have

$$
\begin{aligned}
&\left|u_{x}(0)\right| \lesssim \int\left|\omega_{x}(y)\right||\log (y)| d y \lesssim\left(\int \omega_{x}^{2} \psi_{\alpha}\right)^{1 / 2}\left(\int \psi_{\alpha}^{-1}|\log y|^{2}\right)^{1 / 2} \\
& \lesssim E(t)\left(\alpha^{3} \int|\log y|^{2}\left(|y|^{1+\alpha}+|y|^{1-3 \alpha}\right)^{-1} d y\right)^{1 / 2} \lesssim E(t)\left(\alpha^{3} \alpha^{-1}\right)^{1 / 2} \lesssim \alpha E(t) .
\end{aligned}
$$

The normalization condition (4.9) implies

$$
\left|c_{\omega}(t)\right|=\left|u_{x}(t, 0)\right| \leq C_{3} \alpha E(t), \quad\left|c_{l}(t)\right|=\left|a u_{x}(0)\right| \leq C_{3}|a| \alpha E(t),
$$

for some absolute constant $C_{3}>0$.

Finally, we perform the bootstrap argument. We first choose $C_{2}=16 C$, where $C$ is defined in (4.38), and then choose $C_{1}$ small such that

$$
C_{2}=16 C, \quad C C_{1}+C C_{2} C_{1}+C_{3} C_{2} C_{1}+C_{1}<1 / 16 .
$$

Using the bootstrap argument and an argument similar to that in (2.30), we obtain that if

$$
|a| \alpha<C_{1}, \quad E(0)<C_{2}|a| \alpha,
$$

then we must have $E(t)<C_{2}|a| \alpha<C_{2} C_{1}$. This is because if the right hand side of (4.38) at $E(t)=C_{2}|a| \alpha$ is given by

$$
E(t)^{2}\left(-\frac{3}{8}+C|a| \alpha+\frac{C|a| \alpha}{E(t)}+C E(t)\right)=E(t)^{2}\left(-\frac{3}{8}+\left(C+C C_{2}\right)|a| \alpha+\frac{C}{C_{2}}\right)<-\frac{1}{4} E^{2}(t)<0 .
$$

Finally, we will show that $c_{\omega}+\bar{c}_{\omega}<0$. The bootstrap results, (4.39) and (4.40), imply that

$$
\begin{aligned}
& \left|c_{\omega}(t)\right|,\left|c_{l}(t)\right|<C_{3} E(t)<C_{3} C_{2} C_{1}<\frac{1}{16}, \\
\Rightarrow & \bar{c}_{\omega}+c_{\omega}(t)=-1+c_{\omega}(t)<-\frac{1}{2}, \quad \bar{c}_{l}+c_{l}(t)=\frac{1}{\alpha}-2 a+c_{l}(t)>\frac{1}{2 \alpha}-\frac{1}{16} \geq \frac{1}{4 \alpha},
\end{aligned}
$$

As a result, we can choose small initial perturbation $\omega_{0}$ with $E(0)<C_{2}|a| \alpha$. Moreover, we choose $\omega_{0}$ in such a way that it modifies $\omega_{\alpha}$ at the far field to make the initial data $\bar{\omega}+\omega_{0}$ have compact support. The bootstrap result and $\bar{c}_{\omega}+c_{\omega}(\tau)<-1 / 2<0$ imply that the physical solution blows up in finite time.

4.1.8. Convergence to the steady state. Using the same argument as we did for small $|a|$ and the a-priori estimate (4.42), we can prove that there exists

$$
c_{l, \infty} \geq(4 \alpha)^{-1}, c_{\omega, \infty}<-1 / 2, \omega_{\infty} \in H^{1}\left(\psi_{\alpha}\right) \cap L^{2}\left(\varphi_{\alpha}\right), u_{\infty, x}=H \omega_{\infty},
$$

which satisfy the self-similar equation (4.5) in $L^{2}\left(\varphi_{\alpha}\right)$ and in the dynamical rescaling space, $\omega(t)+\omega_{\alpha}$ converges to $\omega_{\infty}$ exponentially fast in $L^{2}\left(\varphi_{\alpha}\right)$. Therefore, the physical solution develops a focusing $\left(c_{l, \infty}>0\right)$ self-similar blowup in finite time.

4.2. Finite Time Blowup on Circle. In this subsection, we consider the De Gregorio model on $S^{1}$

$$
\begin{aligned}
\omega_{t}+a u \omega_{x} & =u_{x} \omega \quad x \in[-\pi / 2, \pi / 2], \\
u_{x} & =H_{c} \omega,
\end{aligned}
$$

where $\omega, u$ are $\pi$-periodic and $H_{c}$ is the Hilbert transform on the circle

$$
u_{x}=H_{c} \omega=\frac{1}{\pi} \int_{-\pi / 2}^{\pi / 2} \omega(y) \cot (x-y) d y .
$$

Our goal is to prove Theorem [1.4. The proof is based on the comparison of the Hilbert transform on the real line and on $S^{1}$, and on the control of the support of the vorticity. Since the blow-up is focusing, i.e. $c_{l}>0$, if the initial data has finite support, we can show that the support of the solution at blow-up time remains finite. Moreover, we show that the difference between the velocities generated by different Hilbert transforms can be arbitrarily small by choosing initial data with small support. Therefore, the blowup mechanism on the real line can directly lead to the blowup on the circle. 
ON THE FINITE TIME BLOWUP OF THE DE GREGORIO MODEL FOR THE 3D EULER EQUATION 33

4.2.1. Dynamical Rescaling. We consider the following dynamical rescaling of (4.43)

$$
\Omega(x, \tau)=C_{\omega}(\tau) \omega\left(C_{l}(\tau) x, t(\tau)\right), \quad U_{x}(x, \tau)=C_{\omega}(\tau) u_{x}\left(C_{l}(\tau) x, t(\tau)\right) .
$$

Denote by $S(\tau)$ the size of support of $\Omega(\cdot, t(\tau))$, i.e. $\operatorname{supp}(\Omega)=[-S(\tau), S(\tau)]$. Or equivalently, $C_{l}(\tau) S(\tau)$ is the size of $\operatorname{supp}(\omega)$. We will choose $C_{l}(0) S(0)$ to be small and show that $C_{l}(\tau) S(\tau)$ remains small up to the blowup time. We have

$$
\begin{aligned}
U_{x}(x, \tau) & =C_{\omega}(\tau) u_{x}\left(C_{l} x, t(\tau)\right)=\frac{1}{\pi} C_{\omega}(\tau) \int_{-\pi / 2}^{\pi / 2} \omega(y, t(\tau)) \cot \left(C_{l}(\tau) x-y\right) d y \\
& =\frac{1}{\pi} C_{\omega}(\tau) \int_{-C_{l}(\tau) S(\tau)}^{C_{l}(\tau) S(\tau)} \omega(y, t(\tau)) \cot \left(C_{l}(\tau) x-y\right) d y \\
& =\frac{C_{\omega}(\tau)}{\pi} \int_{-S(\tau)}^{S(\tau)} \omega\left(C_{l} y, t(\tau)\right) \cot \left(C_{l}(\tau) x-C_{l}(\tau) y\right) C_{l}(\tau) d y \\
& =\frac{1}{\pi} \int_{-S(\tau)}^{S(\tau)} \Omega(y, \tau) \cot \left(C_{l}(\tau) x-C_{l}(\tau) y\right) C_{l}(\tau) d y \triangleq H_{\tau} \Omega(x) .
\end{aligned}
$$

We introduce the time-dependent Hilbert transform $H_{\tau}$. The corresponding $U$ is given by

$$
\begin{aligned}
U(x, \tau) & =\int_{0}^{x} U_{x}(y, \tau) d y=\frac{1}{\pi} \int_{-S(\tau)}^{S(\tau)} \Omega(y) \log \left|\sin \left(C_{l}(\tau) x-C_{l}(\tau) y\right)\right| d y \\
& =\frac{1}{\pi} \int_{0}^{S(\tau)} \Omega(y) \log \left|\frac{\sin \left(C_{l}(\tau) x-C_{l}(\tau) y\right)}{\sin \left(C_{l}(\tau) x+C_{l}(\tau) y\right)}\right| d y .
\end{aligned}
$$

With this notation, we can formulate the dynamical rescaling equation below

$$
\begin{aligned}
\Omega_{\tau}+\left(c_{l} x+a U\right) \Omega_{x} & =\left(c_{\omega}+U_{x}\right) \Omega, \\
U_{x} & =H_{\tau} \Omega .
\end{aligned}
$$

To simplify our notations, we still denote $\Omega, U, \tau$ in the dynamical rescaling space by $\omega, u, t$ i.e.

$$
(\Omega, U, \tau) \rightarrow(\omega, u, t)
$$

4.2.2. The bootstrap assumption. We make the following bootstrap assumption.

(a) Support of $\omega$ in the physical space : For all $t>0$ we have

$$
C_{l}(t) S(t)<\frac{\pi}{4}
$$

(b) Bounded perturbation : Let $\varphi_{\alpha}, \psi_{\alpha}$ be the weights in (4.4) and $C_{2}$ be the constant in (4.40). We assume

$$
\begin{aligned}
& E^{2}(t)=\left\langle\left(\omega-\omega_{\alpha}\right)^{2}, \varphi_{\alpha}\right\rangle+\mu\left\langle\left(\omega_{x}-\omega_{\alpha, x}\right)^{2}, \psi_{\alpha}\right\rangle<\left(C_{2} a \alpha\right)^{2}<1, \\
& \left|c_{\omega}(t)+1\right|<\frac{1}{2}, \quad\left|c_{l}(t)-\frac{1}{\alpha}\right|<\frac{1}{2 \alpha}
\end{aligned}
$$

where $c_{l}, c_{\omega}, \omega$ is the solution of (4.47) at time $t$ and $\omega-\omega_{\alpha}, c_{\omega}(t)+1$ and $c_{l}(t)-\frac{1}{\alpha}$ can be regarded as perturbation. From (4.13) and the definition of $\omega_{\alpha}$ in (4.2), we know

$$
|x|^{-1-\alpha}+|x|^{-1+\alpha} \lesssim \alpha \varphi_{\alpha}, \quad\left|\omega_{\alpha}(x)\right| \lesssim \alpha|x|^{\alpha} \wedge|x|^{-\alpha}
$$

As a result of (4.49), the solution $\omega$ satisfies

$$
\left\langle\omega^{2},|y|^{-1-\alpha}+|y|^{-1+\alpha}\right\rangle \lesssim \alpha\left\langle\left(\omega-\omega_{\alpha}\right)^{2}, \varphi_{\alpha}\right\rangle+\left\langle\omega_{\alpha}^{2},|y|^{-1-\alpha}+|y|^{-1+\alpha}\right\rangle \lesssim \alpha 1 .
$$

Similarly, from (4.13), the definition of $\omega_{\alpha}$ in (4.2) and (4.49), we have

$$
\left\langle\omega_{x}^{2},|y|^{1-\alpha}+|y|^{1+\alpha}\right\rangle \lesssim\left\langle\left(\omega_{x}-\omega_{\alpha, x}\right)^{2}+\omega_{\alpha, x}^{2},|y|^{1-\alpha}+|y|^{1+\alpha}\right\rangle \lesssim \alpha, \mu 1 .
$$

We remark that the constants in the upper bound of (4.50) and (4.51) are bounded by $C \alpha, C \alpha^{3}\left(\mu^{-1}+\right.$ 1) for some absolute constant $C$, respectively, but we do not need this smallness. 
4.2.3. Control of the support. We choose the same weights $\varphi_{\alpha}, \psi_{\alpha}$ as in (4.4) for later energy estimate. The evolution of the support of $\Omega$ in (4.47), i.e. $S(t)$, is given by

$$
\frac{d}{d t} S(t)=c_{l}(t) S(t)+a U(S(t), t) .
$$

Firstly, we show that $U$ has a sublinear growth if $\left\langle\omega^{2}, \varphi_{\alpha}\right\rangle$ is bounded. Using (4.46) and the Cauchy-Schwartz inequality, we get

$$
\mid U\left(S(t) \mid \lesssim\left\langle\omega^{2},|y|^{-1+\alpha}\right\rangle^{1 / 2}\left(\int_{0}^{S(t)}|y|^{1-\alpha}\left(\log \left|\frac{\sin \left(C_{l}(t) S(t)-C_{l}(t) y\right)}{\sin \left(C_{l}(t) S(t)+C_{l}(t) y\right)}\right|\right)^{2} d y\right)^{1 / 2}\right.
$$

Since $0<y<S(t)$ and $(|y|+S(t)) C_{l}(t)<\pi / 2$ (4.48), we can use

to obtain for any $y \in[0, S(t)]$

$$
\frac{2}{\pi} x \leq \sin (x) \leq x, \quad x \in[0, \pi / 2]
$$

$$
\left(\log \left|\frac{\sin \left(C_{l}(t) S(t)-C_{l}(t) y\right)}{\sin \left(C_{l}(t) S(t)+C_{l}(t) y\right)}\right|\right)^{2} \lesssim 1+\left(\log \left|\frac{C_{l}(t)(S(t)-y)}{C_{l}(t)(S(t)+y)}\right|\right)^{2}=1+\left(\log \left|\frac{S(t)-y}{S(t)+y}\right|\right)^{2} .
$$

Substituting the above estimate in the integral in (4.53), we obtain

$$
\begin{aligned}
\mid U(S(t) \mid & \lesssim\left\langle\omega^{2},|y|^{-1+\alpha}\right\rangle^{1 / 2}\left(\int_{0}^{S(t)}|y|^{1-\alpha}\left(1+\left(\log \left|\frac{S(t)-y}{S(t)+y}\right|\right)^{2}\right) d y\right)^{1 / 2} \\
& \lesssim\left\langle\omega^{2},|y|^{-1+\alpha}\right\rangle^{1 / 2}\left(|S(t)|^{2-\alpha} \int_{0}^{1}|z|^{1-\alpha}\left(1+\left(\log \left|\frac{1-z}{1+z}\right|\right)^{2}\right) d z\right)^{1 / 2},
\end{aligned}
$$

where we have used the change of variable $y=S(t) z$ to get the second inequality. Using the above estimate and (4.50), we obtain

$$
\mid U\left(S(t) \mid \lesssim\left\langle\omega^{2},|y|^{-1+\alpha}\right\rangle^{1 / 2} S(t)^{1-\alpha / 2} \lesssim \alpha S(t)^{1-\alpha / 2} .\right.
$$

Substituting the above estimate in (4.52), we yield

$$
\frac{d}{d t} S(t) \leq c_{l}(t) S(t)+C(a, \alpha) S(t)^{1-\alpha / 2}
$$

where the constant $C(a, \alpha)$ only depends on $a, \alpha$. Recall

$$
C_{l}(t)=C_{l}(0) \exp \left(-\int_{0}^{t} c_{l}(s) d s\right) \text {. }
$$

Denote $P(t) \triangleq C_{l}(t) S(t)$. (4.54) implies the following differential inequality

$$
\frac{d}{d t} P(t)=\frac{d}{d t}\left(C_{l}(t) S(t)\right) \leq C(a, \alpha) C_{l}(t)^{\alpha / 2}\left(C_{l}(t) S(t)\right)^{1-\alpha / 2}=C(a, \alpha) C_{l}(t)^{\alpha / 2} P(t)^{1-\alpha / 2} .
$$

Using the bootstrap assumption $c_{l}(t)>\frac{1}{2 \alpha}$ (4.49), we have

$$
C_{l}(t) \leq C_{l}(0) \exp \left(-\frac{t}{2 \alpha}\right) .
$$

From the above estimate and (4.55), we further obtain

$$
\begin{aligned}
& \frac{d}{d t} P(t)^{\alpha / 2} \leq C(a, \alpha) C_{l}(t)^{\alpha / 2} \leq C(a, \alpha) C_{l}(0)^{\alpha / 2} \exp \left(-\frac{t}{4}\right), \\
& \Rightarrow P(t)^{\alpha / 2} \leq P(0)^{\alpha / 2}+C(a, \alpha) C_{l}(0)^{\alpha / 2} \int_{0}^{t} \exp \left(-\frac{s}{4}\right) d s<P(0)^{\alpha / 2}+C(a, \alpha) C_{l}(0)^{\alpha / 2},
\end{aligned}
$$

where the $C(a, \alpha)$ only depends on $a, \alpha$ and may vary from line to line. Recall $P(0)=C_{l}(0) S(0)$. As a result of the above estimate, we obtain

$$
P(t)^{\alpha / 2} \leq\left(1+C(a, \alpha) S(0)^{-\alpha / 2}\right) P(0)^{\alpha / 2} \Rightarrow P(t) \leq C(a, \alpha, S(0)) P(0),
$$


ON THE FINITE TIME BLOWUP OF THE DE GREGORIO MODEL FOR THE 3D EULER EQUATION 35

where the constant $C(a, \alpha, S(0))$ depends on $a, \alpha$ and $S(0)$.

\subsubsection{Comparison between different Hilbert transforms.}

Lemma 4.6 (Comparison of Hilbert transforms). With the bootstrap assumptions (4.48) and (4.49), for $|x| \leq S(t)$, the difference between $H_{t}$ (4.45) on the circle and the Hilbert transform on the real line $H$ can be controlled by

$$
\begin{aligned}
\left|\left(H_{t} \omega\right)(x)-H \omega(x)\right| & \lesssim_{\alpha} C_{l}(t) S(t), \\
\left|x\left(H_{t} \omega_{x}\right)(x)-x\left(H \omega_{x}\right)(x)\right| & \lesssim_{\alpha, \mu} C_{l}(t) S(t) .
\end{aligned}
$$

Remark 4.7. We only care about $x$ in the support of $\Omega$ since for $x$ outside the support of $\Omega$, $U(x)$ does not enter the equation (4.47).

Proof. It suffices to consider $x \in[0, S(t)]$ due to the symmetry. We only prove the second inequality in (4.57) and the first one can be proved similarly. Firstly, from (4.45), we have (4.58)

$$
\left|x\left(H_{t} \omega_{x}\right)(x)-x\left(H \omega_{x}\right)(x)\right|=\left|\frac{x}{\pi} \int_{-S(t)}^{S(t)} \omega_{x}(y, t)\left(\cot \left(C_{l}(t) x-C_{l}(t) y\right) C_{l}(t)-\frac{1}{x-y}\right) d y\right| .
$$

The bootstrap assumption (4.48) shows that $\left|C_{l}(x-y)\right| \leq \frac{\pi}{2}$ for $|x|,|y| \leq S(t)$. Using the elementary inequality $\left|\frac{1}{z}-\cot z\right| \lesssim \min (|z|, 1) \lesssim 1, \forall|z| \lesssim \frac{\pi}{2}$, we obtain

$$
\left|\cot \left(C_{l}(t) x-C_{l}(t) y\right) C_{l}(t)-\frac{1}{x-y}\right|=C_{l}(t)\left|\cot \left(C_{l}(t) x-C_{l}(t) y\right)-\frac{1}{C_{l}(t)(x-y)}\right| \lesssim C_{l}(t) .
$$

Using the Cauchy-Schwartz inequality, we can estimate (4.58) as follows

$$
\begin{aligned}
& \left|x\left(H_{t} \omega_{x}\right)(x)-x\left(H \omega_{x}\right)(x)\right| \lesssim C_{l}(t)|x| \int_{-S(t)}^{S(t)}\left|\omega_{x}(y, t)\right| d y \\
\leq & C_{l}(t)|x|\left\langle\omega_{x}^{2},|y|^{1-\alpha}+|y|^{1+\alpha}\right\rangle^{1 / 2}\left(\int_{R} \frac{1}{|y|^{1+\alpha}+|y|^{1-\alpha}} d y\right)^{1 / 2} .
\end{aligned}
$$

Using $|x| \leq S(t)$ (4.51), we yield

$$
\left|x\left(H_{t} \omega_{x}\right)(x)-x\left(H \omega_{x}\right)(x)\right| \lesssim \alpha, \mu C_{l}(t) S(t) .
$$

4.2.5. Finite time blowup. Recall that for compactly supported solution $\omega(x, \tau)$ with support size $S(\tau)<+\infty$ in the dynamical rescaling equation (4.47), it corresponds to a solution $\omega_{p h y}$ in the physical space (4.43) via

$$
\begin{aligned}
& \omega_{p h y}(x, t)=C_{\omega}(\tau)^{-1} \omega\left(C_{l}(\tau)^{-1} x, t(\tau)\right), \\
& C_{l}(\tau)=C_{l}(0) \exp \left(-\int_{0}^{\tau} c_{l}(s) d s\right), \quad t(\tau)=\int_{0}^{\tau} \exp \left(\int_{0}^{s} c_{\omega}(r) d r\right) d s .
\end{aligned}
$$

The corresponding time in the physical space $t(\tau)$ can be obtained by the above formula. By abusing the notation, we still use $t$ as the time variable in the dynamical rescaling equation. We can rewrite (4.47) as follows

$$
\begin{aligned}
\omega_{t}+\left(c_{l} x+a u\right) \omega_{x} & =\left(c_{\omega}+u_{x}\right) \omega+\left(\left(H_{t} \omega\right)(x)-(H \omega)(x)\right) \omega+a\left((I \omega)(x)-\left(I_{t} \omega\right)(x)\right) x \omega_{x} \\
u_{x} & =H \omega
\end{aligned}
$$

where $u=x(I \omega)(x)$ and the operator $I_{t} \omega, I \omega$ are

$$
\left(I_{t} \omega\right)(x)=\frac{1}{x} \int_{0}^{x}\left(H_{t} \omega\right)(y) d y, \quad(I \omega)(x)=\frac{1}{x} \int_{0}^{x}(H \omega)(y) d y,
$$

i.e. $1 / x$ times the velocity generated by different Hilbert transforms. We choose the following normalization condition

$$
c_{l}(t)=\frac{1}{\alpha}-a\left(H_{t} \omega(t, \cdot)\right)(0), \quad c_{\omega}(t)=1-\left(H_{t} \omega(t, \cdot)\right)(0) .
$$


The difference between (4.9) and the above condition is the Hilbert transform, which can be bounded by (4.57).

For the difference of the Hilbert transform in (4.59), we use (4.57) to obtain the pointwise estimate of $H_{t} \omega-H \omega$ and $x\left(H_{t} \omega-H \omega\right)_{x}$. Similarly, we have the pointwise estimate of $I \omega(x)-$ $I_{t} \omega(x)$ for all $|x|<S(t)$

$$
\begin{aligned}
\left|(I \omega)(x)-\left(I_{t} \omega\right)(x)\right| & \leq \sup _{|y| \leq|x|}\left|\left(H_{t} \omega\right)(x)-H \omega(x)\right| \lesssim \alpha C_{l}(t) S(t), \\
\left|x\left(I \omega-I_{t} \omega\right)_{x}(x)\right| & \leq\left|\left(x\left(I \omega-I_{t} \omega\right)\right)_{x}\right|+\left|\left(I \omega-I_{t} \omega\right)(x)\right| \\
& =\mid\left(\left(H_{t} \omega\right)(x)-H \omega(x)|+|\left(I \omega-I_{t} \omega\right)(x) \mid \lesssim_{\alpha} C_{l}(t) S(t) .\right.
\end{aligned}
$$

For compactly supported initial data $\omega \in C^{\alpha}$ with support size $S(0)$ and

$$
E(0)=\left(\left\langle\left(\omega(0, \cdot)-\omega_{\alpha}\right)^{2}, \varphi_{\alpha}\right\rangle+\mu\left\langle\left(\omega_{x}(0, \cdot)-\omega_{\alpha, x}\right)^{2}, \psi_{\alpha}\right\rangle\right)^{1 / 2}<C_{2}|a| \alpha,
$$

all the estimates of the perturbation $\omega-\omega_{\alpha}$ in the proof of Theorem (1.2) and (4.1) can be derived in the same way with additional terms depending on the difference of the Hilbert transforms, which can be bounded using (4.60). For instance, we consider the $C^{\alpha}$ case on the circle. Under the bootstrap assumption (4.48) and (4.49), the $C^{\alpha}$ differential inequality (4.38) becomes

$$
\frac{1}{2} \frac{d}{d t} E^{2}(t) \leq-\left(\frac{3}{8}-C|a| \alpha\right) E^{2}(t)+C|a| \alpha E(t)+C E^{3}(t)+C_{4}(a, \alpha, \mu) C_{l}(t) S(t) E^{2}(t),
$$

where the constant $C_{4}(a, \alpha, \mu)$ depends on $a, \alpha, \mu$. Using the control of the support (4.56), we have

$$
C_{l}(t) S(t)=P(t) \leq C(a, \alpha, S(0)) P(00)=C(a, \alpha, S(0)) C_{l}(0) S(0),
$$

which implies

$$
\begin{aligned}
\frac{1}{2} \frac{d}{d t} E^{2}(t) & \leq-\left(\frac{3}{8}-C|a| \alpha\right) E^{2}(t)+C|a| \alpha E(t)+C E^{3}(t) \\
& +C_{5}(a, \alpha, \mu, S(0)) C_{l}(0) S(0) E^{2}(t),
\end{aligned}
$$

where the constant $C_{5}(a, \alpha, \mu, S(0))$ depends on $a, \alpha, \mu, S(0)$. Note that $C_{l}(0)$ is independent of the initial data $\omega(0, \cdot)$ in the dynamical rescaling space and only depends on how we rescale $\omega(0, \cdot)$ to get the data in the physical space. We first choose compactly supported $\omega(0, \cdot)$ that satisfies

$$
E(0)=\left(\left\langle\left(\omega(0, \cdot)-\omega_{\alpha}\right)^{2}, \varphi_{\alpha}\right\rangle+\mu\left\langle\left(\omega_{x}(0, \cdot)-\omega_{\alpha, x}\right)^{2}, \psi_{\alpha}\right\rangle\right)^{1 / 2}<C_{2}|a| \alpha .
$$

In this case, $S(0)<+\infty$. Then we choose $C_{l}(0)$ sufficiently small such that

$$
\left(C(a, \alpha, S(0))+C_{5}(a, \alpha, \mu, S(0))+1\right) C_{l}(0) S(0)<\frac{1}{16} .
$$

Under the bootstrap assumption (4.48) and (4.49), we plug the above inequality in (4.61) and (4.62) to get

$$
\begin{aligned}
C_{l}(t) S(t) & \leq C(a, \alpha, S(0)) C_{l}(0) S(0)<\frac{1}{16}<\frac{\pi}{4}, \\
\frac{1}{2} \frac{d}{d t} E^{2}(t) & \leq-\left(\frac{3}{8}-C|a| \alpha\right) E^{2}(t)+C|a| \alpha E(t)+C E^{3}(t)+\frac{1}{16} E^{2}(t) .
\end{aligned}
$$

The above differential inequality differs from (4.38) only by the extra term, $E(t)^{2} / 16$. (4.41) shows that the right hand side of the above differential inequality at $E(t)=C_{2}|a| \alpha$ is less than

$$
E^{2}(t)\left(-\frac{1}{4}+\frac{1}{16}\right)<0 .
$$

Since this constant $C_{2}$ is the same as that in (4.49), it follows that the bootstrap assumption (4.48) and (4.49) can be continued. The remaining steps are exactly the same as those in the proof of Theorem 4.1 and we conclude the proof of Theorem 1.4 for the $C^{\alpha}$ case. For the case when $a$ is a small positive constant, we can also prove a similar result. We will omit the proof here. 
ON THE FINITE TIME BLOWUP OF THE DE GREGORIO MODEL FOR THE 3D EULER EQUATION 37

4.3. Criticality of the $C^{\alpha}$ Regularity. Note that the self-similar solutions in (4.2) all satisfy that $w_{\alpha}$ is odd and $w_{\alpha}$ is negative for $x>0$, we shall consider general initial datum within this class. We remark that the results in this Section only hold true for positive $a$.

4.3.1. The DG equation on the real line. In this Section, we prove Theorem 1.5, which implies that for large positive $a$, the Hölder regularity in the initial datum is crucial for the focusing self-similar blow-up.

Remark 4.8. In the later proof, we choose $C_{1}=(1+0.015) /(1-0.015) \approx 1.03$ in Theorem 1.5. The compact support assumption in Theorem can be relaxed easily by imposing a growth condition on $\omega_{0}$, e.g. $\omega_{0}$ is bounded.

To prove Theorem 1.5 we need the following crucial Lemma, which indicates that the convection term can be stronger than the nonlinear term.

Lemma 4.9. Let $\varepsilon=0.015$. Suppose that $\beta \in[1,2)$ and $a>0$ satisfies

$$
a>\frac{\varepsilon(\beta-1)+1}{(1-\varepsilon)(\beta-1)} .
$$

The following inequality

$$
\int_{0}^{\infty} \frac{u_{x} \omega-a u \omega_{x}}{y^{\beta}} d y \geq 0
$$

holds as long as the left hand side is well-defined and that $\omega$ is odd and non-positive for $x>0$.

Proof. From the assumption of $\omega$, we know

$$
\omega(x)=O\left(|x|^{\alpha}\right), u(x)=O(|x|),
$$

for small $|x|$. Denote by $I$ the integral in (4.64). Using integration by parts and expanding the kernel, we get

$$
\begin{aligned}
I= & \int_{0}^{\infty}(1+a) \frac{u_{x} \omega}{x^{\beta}}-a \beta \frac{u \omega}{x^{1+\beta}} d x=\frac{1}{\pi} \int_{0}^{\infty}(1+a) \frac{\omega(x)}{x^{\beta}} \int_{0}^{\infty} \omega(y)\left(\frac{1}{x-y}-\frac{1}{x+y}\right) d y d x \\
& +\frac{1}{\pi} \int_{0}^{\infty} a \beta \frac{\omega(x)}{x^{1+\beta}} \int_{0}^{\infty} \log \left|\frac{y+x}{y-x}\right| \omega(y) d y d x \\
= & \frac{1+a}{\pi} \int_{0}^{\infty} \int_{0}^{\infty}\left[\frac{1}{x^{\beta}}\left(\frac{1}{x-y}-\frac{1}{x+y}\right)+\frac{a \beta}{1+a} \frac{1}{x^{\beta+1}} \log \left|\frac{y+x}{y-x}\right|\right] \omega(x) \omega(y) d x d y .
\end{aligned}
$$

Since $\omega$ is odd, we can symmetrize the integral kernel

$$
\begin{aligned}
I=\frac{1+a}{2 \pi} \int_{0}^{\infty} \int_{0}^{\infty} & {\left[\frac{1}{x^{\beta}}\left(\frac{1}{x-y}-\frac{1}{x+y}\right)+\frac{a \beta}{1+a} \frac{1}{x^{\beta+1}} \log \left|\frac{y+x}{y-x}\right|\right.} \\
+ & \left.\frac{1}{y^{\beta}}\left(\frac{1}{y-x}-\frac{1}{x+y}\right)+\frac{a \beta}{1+a} \frac{1}{y^{\beta+1}} \log \left|\frac{y+x}{y-x}\right|\right] \omega(x) \omega(y) d x d y .
\end{aligned}
$$

Denote

$$
\tau=\frac{a \beta}{1+a}, \quad s=\frac{y}{x}
$$

We can simplify the integrand as follows

$$
\begin{aligned}
& \frac{1}{x^{\beta}}\left(\frac{1}{x-y}-\frac{1}{x+y}\right)+\frac{1}{y^{\beta}}\left(\frac{1}{y-x}-\frac{1}{x+y}\right) \\
= & \frac{1}{y^{1+\beta}} \frac{y^{1+\beta}}{x^{1+\beta}}\left(\frac{x}{x-y}-\frac{x}{x+y}\right)+\frac{1}{y^{1+\beta}}\left(\frac{y}{y-x}-\frac{y}{x+y}\right) \\
= & \frac{1}{y^{1+\beta}} s^{1+\beta}\left(\frac{1}{1-s}-\frac{1}{1+s}\right)+\frac{1}{y^{1+\beta}}\left(\frac{s}{s-1}-\frac{s}{s+1}\right)=-\frac{1}{y^{1+\beta}}\left(s^{1+\beta}-1\right) \frac{2 s}{s^{2}-1}, \\
& \frac{1}{x^{\beta+1}} \log \left|\frac{y+x}{y-x}\right|+\frac{1}{y^{\beta+1}} \log \left|\frac{y+x}{y-x}\right|=\frac{1}{y^{1+\beta}}\left(s^{1+\beta}+1\right) \log \left|\frac{s+1}{s-1}\right| .
\end{aligned}
$$


Then $I$ becomes

$$
I=\frac{1+a}{2 \pi} \int_{0}^{\infty} \int_{0}^{\infty} \frac{1}{y^{1+\beta}}\left(\tau\left(s^{\beta+1}+1\right) \log \left|\frac{s+1}{s-1}\right|-\left(s^{1+\beta}-1\right) \frac{2 s}{s^{2}-1}\right) \omega(x) \omega(y) d x d y .
$$

Denote

$$
F(s, \beta) \triangleq \frac{1-s^{1+\beta}}{1+s^{1+\beta}} \frac{2 s}{1-s^{2}}\left(\log \left|\frac{s+1}{s-1}\right|\right)^{-1}, \quad s \in[0,1], \beta \in[1,2] .
$$

We have the following basic property for $F(s, \beta)$.

Lemma 4.10. Assume that $s \in[0,1], \beta \in[1,2]$. Then (a) $F(s, \beta)$ is monotonically increasing with respect to $\beta$. (b) For any $s \in[0,1]$, we have

$$
F(s, 1) \leq 1, \quad F(s, \beta)<1+0.015(\beta-1) \quad \forall \beta \in(1,2] .
$$

We defer the proof of the above Lemma to the Appendix. Let $\varepsilon=0.015$. As a result, if

$$
\tau=\frac{a \beta}{1+a}>1+\varepsilon(\beta-1) \Longleftrightarrow a>\frac{\varepsilon(\beta-1)+1}{(1-\varepsilon)(\beta-1)},
$$

then $I$ in (4.65) is non-negative

$$
I=\frac{1+a}{2 \pi} \int_{0}^{\infty} \int_{0}^{\infty} \frac{1}{y^{1+\beta}}\left(\left(s^{\beta+1}+1\right) \log \left|\frac{s+1}{s-1}\right|(\tau-F(s, \beta))\right) \omega(x) \omega(y) d x d y \geq 0,
$$

where we have used $\omega(x) \omega(y) \geq 0 \forall x, y \geq 0$.

Now, we are in a position to prove Theorem 1.5 .

Proof of Theorem 1.5. Denote $a_{0}=\frac{1+\varepsilon}{1-\varepsilon}$. If $1 \geq \alpha>a_{0} / a$, we get

$$
a \alpha>a_{0}=\frac{1+\varepsilon}{1-\varepsilon} \geq \frac{\varepsilon \alpha+1}{1-\varepsilon} \Rightarrow a>\frac{1+\varepsilon \alpha}{(1-\varepsilon) \alpha} .
$$

Therefore, we can choose $1 \leq \beta<\alpha+1$, e.g. $\beta=1+\alpha-\delta$ for some sufficiently small $\delta$, such that

$$
a>\frac{1+\varepsilon(\beta-1)}{(1-\varepsilon)(\beta-1)},
$$

i.e. $(a, \beta)$ satisfies the assumption (4.63) in Lemma 4.9. For $\omega \in C^{\alpha}$ with compact support (or some growth condition at the far field), we have $\omega(y)|y|^{-\beta} \in L^{1}$. Using (1.3) and Lemma 4.9. we get

$$
\frac{d}{d t} \int_{0}^{\infty} \frac{\omega(t, y)}{y^{\beta}} d y=\int_{0}^{\infty} \frac{u_{x} \omega-a u \omega_{x}}{y^{\beta}} d y \geq 0
$$

Note that $\omega$ is odd and non-positive for $x>0$. We yield

$$
0 \geq \int_{0}^{\infty} \frac{\omega(t, y)}{y^{\beta}} d y \geq \int_{0}^{\infty} \frac{\omega(0, y)}{y^{\beta}} d y \Rightarrow\left\|\frac{\omega(t, \cdot)}{|y|^{\beta}}\right\|_{1} \leq\left\|\frac{\omega(0, \cdot)}{|y|^{\beta}}\right\|_{1}<+\infty
$$

for all $t>0$. If $\omega$ blows up in a self-similar fashion, i.e.

$$
\omega(t) \rightarrow(T-t)^{-1} \Omega\left(\frac{x}{(T-t)^{c_{l}}}\right),
$$

in some suitable functional space, (the convergence can be measured in the dynamical rescaling space), we can plug the self-similar blowup ansatz in $I(t)$ to yield

$$
\left\|\frac{\omega(t, \cdot)}{|y|^{\beta}}\right\|_{1} \rightarrow(T-t)^{-1} \int_{\mathbf{R}}\left|\Omega\left(\frac{x}{(T-t)^{c_{l}}}\right)\right||x|^{-\beta} d x=(T-t)^{-1+c_{l}-\beta c_{l}} \int_{\mathbf{R}}\left|\frac{\Omega(x)}{x^{\beta}}\right| d x
$$

as $t \rightarrow T$. Since $\left\|\frac{\omega(t, \cdot)}{|y|^{\beta}}\right\|_{1}$ is bounded uniformly in $t$, we get

$$
-1+c_{l}-\beta c_{l} \geq 0 \Rightarrow c_{l} \leq-\frac{1}{\beta-1},
$$

for any $\beta<1+\alpha$. Letting $\beta \rightarrow \alpha+1$, we get $c_{l} \leq-\alpha^{-1}$. 
ON THE FINITE TIME BLOWUP OF THE DE GREGORIO MODEL FOR THE 3D EULER EQUATION 39

4.3.2. DG equation on the circle. For the DG equation the circle, we can prove a stronger result.

Theorem 4.11. Suppose that $\omega \in C^{\alpha}$ is odd, $\pi$ periodic, $\omega \leq 0$ for $x \in(0, \pi / 2)$ and $1 \geq \alpha>$ $a_{0} / a$. Then the result in Theorem 1.5 holds true. Furthermore, $u_{x}(0),\|\omega\|_{1}$ do not blow up at the blowup time $T$, if it exists, and grow at most exponentially fast.

Remark 4.12. From our numerical experiments, we found that for various initial data, $u_{x}(0, t)=$ $\max u_{x}(x, t)$ for some finite time. Theorem 4.11 gives a strong indication that $u_{x}$ is bounded from above if $u_{x}(0)$ is bounded. From

$$
\frac{d}{d t} \max \omega \leq\left(\max u_{x}\right) \max \omega
$$

$(\max \omega>0)$ and the assumption $u_{x}(0, t)=\max u_{x}(x, t)$, we obtain the boundedness of $\max \omega$ for all time. Since $\omega$ is odd, we get $\|\omega\|_{\infty}=\max \omega$ is bounded globally. Applying the BKM-type blowup criterion yields the global well-posedness.

The following Lemma is an analogy of Lemma 4.9 on the circle.

Lemma 4.13. With the assumption as Lemma 4.9 ,

$$
\int_{0}^{\pi / 2}\left(u_{x} \omega-a u \omega_{x}\right)(\cot y)^{\beta} d y \geq 0 .
$$

The proof is similar to that of Lemma 4.9 and we defer it to the Appendix.

Proof of Theorem 4.13. Using Lemma 4.13 and an argument similar to that in the proof of Theorem 1.5, we derive

$$
\int_{0}^{\pi / 2} \omega_{0}(\cot y)^{\beta} \leq \int_{0}^{\pi / 2} \omega(\cot y)^{\beta} \leq 0 \Rightarrow|| \omega(t, \cdot)|\cot y|^{\beta}\left\|_{L^{1}} \leq|| \omega_{0}|\cot y|^{\beta}\right\|_{1}<+\infty,
$$

for some $1<\beta<1+\alpha$. Next, we estimate the $L^{1}$ norm of $\omega$. Integrating (4.43) from $\pi / 2$ to $\pi$ yields

$$
\begin{aligned}
& \frac{d}{d t} \int_{0}^{\pi / 2} \omega\left(y+\frac{\pi}{2}\right) d y=-(1+a) \int_{0}^{\pi / 2} \int_{0}^{\pi / 2} \omega\left(x+\frac{\pi}{2}\right) \omega\left(y+\frac{\pi}{2}\right) \cot (x+y) d x d y \\
= & -2(1+a) \int_{0 \leq x \leq y \leq \pi / 2} \omega\left(x+\frac{\pi}{2}\right) \omega\left(y+\frac{\pi}{2}\right) \cot (x+y) d x d y . \\
= & -2(1+a) \int_{0}^{\pi / 2} \omega\left(x+\frac{\pi}{2}\right)\left(\int_{x}^{\pi / 2} \omega\left(y+\frac{\pi}{2}\right) \cot (x+y) d y\right) d x .
\end{aligned}
$$

Note that $\omega \geq 0$ on $(\pi / 2, \pi)$ and $\cot (x+y) \geq 0$ if $x+y \leq \pi / 2$. We yield

$$
-\int_{x}^{\pi / 2} \omega(y+\pi / 2) \cot (x+y) d y \leq-\int_{x \vee(\pi / 2-x)}^{\pi / 2} \omega(y+\pi / 2) \cot (x+y) d y .
$$

For $x \in[0, \pi / 2], x \vee(\pi / 2-x) \leq y \leq \pi / 2$, we have

$0 \leq x+y-\pi / 2 \leq y \leq \pi / 2, \pi / 4 \leq y \Rightarrow-\cot (x+y)=\tan (x+y-\pi / 2) \leq \tan y \leq(\tan y)^{\beta}$, where $\beta \geq 1$ satisfies the assumption in Lemma 4.9, It follows that

$$
\begin{aligned}
& -\int_{x}^{\pi / 2} \omega(y+\pi / 2) \cot (x+y) d y \leq \int_{x \vee(\pi / 2-x)}^{\pi / 2} \omega(y+\pi / 2) \tan y d y \\
\leq & \int_{0}^{\pi / 2} \omega(y+\pi / 2)(\tan y)^{\beta} d y \leq|| \omega(t, \cdot)|\cot y|^{\beta}\left\|_{L^{1}} \leq|| \omega_{0}|\cot y|^{\beta}\right\|_{L^{1}} .
\end{aligned}
$$

Plugging the above estimate in (4.68) implies

$$
\frac{d}{d t} \int_{0}^{\pi / 2} \omega(y+\pi / 2) d y \lesssim(1+a)|| \omega_{0}|\cot y|^{\beta} \|_{L^{1}} \int_{0}^{\pi / 2} \omega(y+\pi / 2) d y
$$


Note that $\omega \geq 0$ on $[\pi / 2, \pi]$ and $\|\omega\|_{L^{1}}=2 \int_{0}^{\pi / 2} \omega(y+\pi / 2)$. Using the Gronwall inequality, we obtain

$$
\|\omega(t, \cdot)\|_{L^{1}} \leq \exp \left(C(1+a)\left\|\omega_{0}|\cot y|^{\beta}\right\|_{L^{1}} t\right)\left\|\omega_{0}\right\|_{L^{1}}
$$

where $C$ is some universal constant. Interpolating $\|\omega\|_{L^{1}}$ and $\|\omega \mid \cot y\|^{\beta} \|_{L^{1}}$ gives

$$
\left|u_{x}(0)\right|=\left|\frac{2}{\pi} \int_{0}^{\pi / 2} \omega \cot y d y\right| \lesssim|| \omega \|\left._{L^{1}}^{1-1 / \beta}|| \omega|\cot y|^{\beta}\right|_{L^{1}} ^{1 / \beta} \lesssim K_{1} \exp \left(K_{2} t\right)
$$

where $K_{1}, K_{2}$ depend on the initial datum and $a, \alpha$ only.

Finally, we state a result for $a=1$.

Proposition 4.14. For $a=1$, suppose that $\omega_{0} \in H^{s}\left(S^{1}\right), s>5 / 2, \omega_{0, x}(0)=0$ and $\omega_{0} \leq 0$ for $x \in(0, \pi / 2)$, then $u_{x}(t, 0),\|\omega\|_{1}$ do not blow up at the blowup time $T<+\infty$, if it exists.

Proof. Since $\omega \in H^{s}, s>5 / 2$, we have local well-posedness and that $\omega(t, \cdot) \in C^{2}$ by the Sobolev embedding. Note that for $a=1, \omega_{x}(t, 0) \equiv \omega_{x}(0,0)=0$. Since $\omega(t, 0)=\omega_{x}(t, 0)=0$, we have $\omega(t, x)=O\left(x^{2}\right)$ near $x=0$. Define

$$
I \triangleq \int_{0}^{\pi / 2}\left(u_{x} \omega-u \omega_{x}\right)(\cot y)^{\beta} d y
$$

for any $\beta \in(1,3)$. In particular, for $\beta=2.2$, using an argument similar to that in the proof of Lemma 4.13, one can show that $I>0$. The boundedness of $u_{x}(0),\|\omega\|_{1}$ follows by using an argument similar to that in the proof of Theorem 4.11.

Remark 4.15. The regularity of $\omega_{0}$ can be relaxed easily and we do not explore it.

\section{Finite Time Blowup for Negative $a$ With $C^{\infty}$ initial data}

For the sake of completeness, we state the finite time blowup result of (1.3) for negative $a$ with smooth initial data.

Theorem 5.1. Let $\omega \in C_{c}^{\infty}(\mathbf{R})$ or $\omega \in C^{\infty}\left(S^{1}\right)$ be an odd function such that $u_{x}(0)=H \omega(0)>$ 0 . Then (1.3) with $a<0$ develops a singularity in finite time.

The real line case was proved in [1]. We will present a proof for $S^{1}$. We consider $\pi$ periodic and use the Hilbert transform given in (4.44).

Proof. Taking the Hilbert transform on (1.3) yields

$$
\left(u_{x}\right)_{t}=\frac{1}{2}\left(u_{x}^{2}-w^{2}\right)-a H\left(u \omega_{x}\right) .
$$

Note that $\omega(0)=0$. Choosing $x=0$ gives

$$
\frac{d}{d t} u_{x}(t, 0)=\frac{1}{2} u_{x}(t, 0)^{2}-a H\left(u \omega_{x}\right)(t, 0) .
$$

Next we show that $H\left(u \omega_{x}\right)(t, 0) \leq 0$. Since $\omega$ is odd, $\pi$-periodic and smooth locally in time , it admits a decomposition

$$
\omega(t, x)=\sum_{n \geq 1} a_{n}(t) \sin (2 n x), \quad \omega_{x}=\sum_{n \geq 1} 2 n a_{n}(t) \cos (2 n x),
$$

for some $a_{n}(t)$ decays sufficiently fast as $n \rightarrow+\infty$. It is easy to show that

$$
u(t, x)=-\sum_{n \geq 1} \frac{a_{n}}{2 n} \sin (2 n x) .
$$


ON THE FINITE TIME BLOWUP OF THE DE GREGORIO MODEL FOR THE 3D EULER EQUATION 41

Next, we compute $u / \sin (x), \omega_{x} \cos x$. Using telescoping, we get

$$
\begin{aligned}
\frac{\sin (2 n x)}{\sin (x)} & =\sum_{1 \leq k \leq n} 2 \cos ((2 k-1) x), \quad \cos (2 n x) \cos x=\frac{\cos (2 n-1) x+\cos (2 n+1) x}{2}, \\
\Rightarrow \frac{u}{\sin x} & =-\sum_{n \geq 1} \frac{a_{n}}{2 n} \sum_{1 \leq k \leq n} 2 \cos ((2 k-1) x)=-\sum_{k \geq 1} \cos ((2 k-1) x) \sum_{n \geq k} \frac{a_{n}}{n}, \\
\omega_{x} \cos x & =\sum_{n \geq 1} 2 n a_{n} \frac{\cos (2 n-1) x+\cos (2 n+1) x}{2}=\sum_{n \geq 1} \cos ((2 n-1) x)\left(n a_{n}+(n-1) a_{n-1}\right),
\end{aligned}
$$

where $a_{0}=0$ and we have used summation by parts to get the last two identities, which are valid since $a_{n}$ decays sufficiently fast. Using the orthogonality of $\{\cos ((2 n-1) x)\}_{n \geq 1}$ on $L^{2}(-\pi / 2, \pi / 2)$, we derive

$$
H\left(u \omega_{x}\right)(t, 0)=-\frac{1}{\pi} \int_{-\pi / 2}^{\pi / 2} \frac{u}{\sin x} \omega_{x} \cos (x) d x=-\frac{1}{2} \sum_{k \geq 1}\left(\sum_{n \geq k} \frac{a_{n}}{n}\right)\left(k a_{k}+(k-1) a_{k-1}\right) .
$$

Denote

$$
S_{k} \triangleq \sum_{n \geq k} \frac{a_{n}}{n} \quad \forall k \geq 1, \quad S_{0}=0 .
$$

Since $a_{n}$ decays sufficiently fast, so does $S_{n}$. We then have

$$
a_{k}=k\left(S_{k}-S_{k+1}\right) \Rightarrow k a_{k}+(k-1) a_{k-1}=k^{2}\left(S_{k}-S_{k+1}\right)+(k-1)^{2}\left(S_{k-1}-S_{k}\right) .
$$

We can reduce $H\left(u \omega_{x}\right)(t, 0)$ to

$$
\begin{aligned}
H\left(u \omega_{x}\right)(t, 0) & =-\frac{1}{2} \sum_{k \geq 1} S_{k}\left(k^{2}\left(S_{k}-S_{k+1}\right)+(k-1)^{2}\left(S_{k-1}-S_{k}\right)\right) \\
& =-\frac{1}{2} \sum_{k \geq 1} S_{k}^{2}\left(k^{2}-(k-1)^{2}\right)-\frac{1}{2}\left(-\sum_{k \geq 1} S_{k} S_{k+1} k^{2}+\sum_{k \geq 1} S_{k} S_{k-1}(k-1)^{2}\right) \\
& =-\frac{1}{2} \sum_{k \geq 1} S_{k}^{2}\left(k^{2}-(k-1)^{2}\right) \leq 0 .
\end{aligned}
$$

Consequently, for $a<0$, (5.1) implies

$$
\frac{d}{d t} u_{x}(t, 0) \geq \frac{1}{2} u_{x}^{2}(t, 0)
$$

Since $u_{x}(0,0)>0$, it follows that the solution must develop a finite time singularity.

\section{Appendix A.}

A.1. Properties of the Hilbert transform. Throughout this section, without specification, we assume that $\omega$ is smooth and decays sufficiently fast. The general case can be obtained easily by approximation. The following identity is very well known whose proof can be found in, e.g. [10].

Lemma A.1 (The Tricomi identity). We have

$$
H(\omega H \omega)=\frac{1}{2}\left((H \omega)^{2}-\omega^{2}\right) .
$$

The Hilbert transform has a very nice property that it almost commutes with the power $x^{-1}, x$.

Lemma A.2. Suppose that $u_{x}=H \omega$. Then we have

$$
\frac{u_{x}-u_{x}(0)}{x}=H\left(\frac{\omega}{x}\right) \text {, or equivalently }(H \omega)(x)=(H \omega)(0)+x H\left(\frac{\omega}{x}\right) .
$$

Similarly, we have

$$
u_{x x}=H \omega_{x}, x u_{x x}=H\left(x \omega_{x}\right) .
$$


Suppose that in addition $\omega$ is odd. Then we further have

$$
x^{2} u_{x x}=H\left(x^{2} \omega_{x}\right), \quad x u_{x}=H(x \omega), \quad \frac{u_{x x}}{x}=H\left(\frac{\omega_{x}-\omega_{x}(0)}{x}\right) .
$$

If $\omega$ is odd and a piecewise cubic polynomial supported on $[-L, L]$ with $\omega(L)=\omega(-L)=0$ $\left(\omega^{\prime}, \omega^{\prime \prime}\right.$ may not be continuous at $\left.x= \pm L\right)$, then we have

$$
u_{x x x}\left(x^{2}-L^{2}\right)=H\left(\omega_{x x}\left(x^{2}-L^{2}\right)\right) .
$$

We apply A.5 to study the approximate profile $\bar{\omega}$ for $a=1$.

Proof. The identity (A.2) is very well known.

$$
\frac{u_{x}-u_{x}(0)}{x}=\frac{1}{\pi x} P . V . \int \omega(y)\left(\frac{1}{x-y}+\frac{1}{y}\right) d y=\frac{1}{\pi} P . V . \int \frac{\omega(y)}{(x-y) y} d y=H\left(\frac{\omega}{y}\right)(x) .
$$

For (A.3), note that

$$
H \omega_{x}=u_{x x}, \quad H\left(x \omega_{x}\right)(0)=-\frac{1}{\pi} \int \omega_{x} d x=0
$$

From (A.2), we get

$$
H\left(x \omega_{x}\right)(x)=H\left(x \omega_{x}\right)(0)+x\left(H \omega_{x}\right)(x)=x u_{x x}(x) .
$$

For (A.4), if $\omega$ is odd, then we obtain

$$
H\left(x^{2} \omega_{x}\right)(0)=-\frac{1}{\pi} \int x \omega_{x} d x=\frac{1}{\pi} \int \omega d x=0 .
$$

Applying (A.2) again yields

$$
H\left(x^{2} \omega_{x}\right)=H\left(x^{2} \omega_{x}\right)(0)+x H\left(x \omega_{x}\right)=x H\left(x \omega_{x}\right)=x^{2} u_{x x} .
$$

For the second identity, since $\omega$ is odd, we can apply a similar argument to yield

$$
H(x \omega)(0)=-\frac{1}{\pi} \int \omega d x=0 \Rightarrow H(x \omega)(x)=H(x \omega)(0)+x H \omega=x H \omega=x u_{x} .
$$

For the third identity in (A.4), first of all, we have

$$
\omega_{x}=-H u_{x x} .
$$

If $\omega$ is odd, then $u, u_{x x}$ are also odd. $\frac{\omega_{x}-\omega_{x}(0)}{x}$ and $\frac{u_{x x}}{x}$ are $L^{2}$ for $\omega$ smooth with suitable decay at infinity. Using an argument similar to that in the proof of (A.2) implies

$$
\frac{\omega_{x}-\omega_{x}(0)}{x}=-H\left(\frac{u_{x x}}{x}\right) \text {. }
$$

Applying the Hilbert transform on both sides proves the third identity.

Next, we consider (A.5). Since $\omega$ is continuous globally and is a piecewise cubic polynomial on $[-L, L]$, we know

$$
\omega \in H^{1}(\mathbf{R}), \quad \omega \in C^{2,1}[-L, L], \quad \omega_{x}\left(x^{2}-L^{2}\right) \in C^{0,1}(\mathbf{R}) .
$$

Since $\omega \in H^{1}(\mathbf{R})$, we can apply (A.4) to yield

$$
x^{2} u_{x x}=H\left(x^{2} \omega_{x}\right), \quad L^{2} u_{x x}=L^{2} H\left(\omega_{x}\right) \Rightarrow\left(x^{2}-L^{2}\right) u_{x x}=H\left(\omega_{x}\left(x^{2}-L^{2}\right)\right) .
$$

Since $\omega_{x}\left(x^{2}-L^{2}\right)$ is globally Lipschitz, it is in $H^{1}(\mathbf{R})$. By the $L^{2}$ isometry of the Hilbert transform, $u_{x x}\left(x^{2}-L^{2}\right) \in H^{1}(\mathbf{R})$. Hence the derivative commutes with the Hilbert transform $\partial_{x} H\left(\omega_{x}\left(x^{2}-L^{2}\right)\right)=H\left(\partial_{x}\left(\omega_{x}\left(x^{2}-L^{2}\right)\right)\right) \Rightarrow u_{x x x}\left(x^{2}-L^{2}\right)+2 u_{x x} x=H\left(\omega_{x x}\left(x^{2}-L^{2}\right)+2 x \omega_{x}\right)$.

Using the linearity of the Hilbert transform and (A.3), we conclude that

$$
u_{x x x}\left(x^{2}-L^{2}\right)=H\left(\omega_{x x}\left(x^{2}-L^{2}\right)\right)+2 H\left(x \omega_{x}\right)-2 x u_{x x}=H\left(\omega_{x x}\left(x^{2}-L^{2}\right)\right) .
$$


ON THE FINITE TIME BLOWUP OF THE DE GREGORIO MODEL FOR THE 3D EULER EQUATION 43

The following Lemma is used to estimate the velocity. We have applied this Lemma to study the nonlinear stability of a smooth profile.

Lemma A.3. Suppose $u_{x}=H \omega$. (a) We have

$$
\int_{\mathbf{R}} \frac{\left(u_{x}-u_{x}(0)\right) \omega}{x} d x=\frac{\pi}{2}\left(u_{x}^{2}(0)+\omega^{2}(0)\right) \geq 0 .
$$

Furthermore, if $\omega$ is odd (so is $u_{x x}$ due to the symmetry of Hilbert transform), we have

$$
\int_{\mathbf{R}} \frac{\left(u_{x}-u_{x}(0)\right) \omega}{x^{3}} d x=\frac{\pi}{2}\left(\omega_{x}^{2}(0)-u_{x x}^{2}(0)\right)=\frac{\pi}{2} \omega_{x}^{2}(0) \geq 0 .
$$

In particular, (A.6) vanishes if $u_{x}(0)=\omega(x)=0$; (A.7) vanishes if $\omega_{x}(0)=0$.

(b) We have

$$
\int_{\mathbf{R}} u_{x x} \omega_{x} x d x=0
$$

(c) The Hardy inequality: Suppose that $\omega$ is odd and $\omega_{x}(0)=0$. For $p=2,4$, we have

$$
\int \frac{\left(u-u_{x}(0) x\right)^{2}}{|x|^{p+2}} d x \leq\left(\frac{2}{p+1}\right)^{2} \int \frac{\left(u_{x}-u_{x}(0)\right)^{2}}{|x|^{p}} d x=\left(\frac{2}{p+1}\right)^{2} \int \frac{\omega^{2}}{|x|^{p}} d x .
$$

Proof of (A.6). Note that

$$
u_{x}=H \omega, \quad u_{x}(0)=H \omega(0)=-\frac{1}{\pi} \int \frac{\omega}{x} d x .
$$

Using Lemma A.1 we get

$$
\begin{aligned}
\int \frac{\left(u_{x}-u_{x}(0)\right) \omega}{x} d x & =\int \frac{\omega \cdot H \omega}{x} d x-u_{x}(0) \int \frac{\omega}{x} d x=-\pi H(\omega \cdot H \omega)(0)+\pi u_{x}(0) \cdot u_{x}(0) \\
& =\frac{\pi}{2}\left(\omega^{2}(0)-u_{x}^{2}(0)\right)+\pi u_{x}^{2}(0)=\frac{\pi}{2}\left(\omega^{2}(0)+u_{x}^{2}(0)\right) .
\end{aligned}
$$

If $\omega(0)=0$, the above equality is reduced to $\frac{\pi}{2} u_{x}^{2}(0)$.

Proof of (A.7). If $\omega$ is odd and smooth, then $\omega / x$ is even and smooth and $H(\omega / x)$ is odd. Using (A.2) and Lemma A.1, we have

$$
\begin{aligned}
\int \frac{\left(u_{x}-u_{x}(0)\right) \omega}{x^{3}} d x & =\int \frac{1}{x} \frac{\omega}{x} H\left(\frac{\omega}{x}\right) d x=-\pi H\left(\frac{\omega}{x} H\left(\frac{\omega}{x}\right)\right)(0) \\
& =\frac{\pi}{2}\left\{\left(\frac{\omega}{x}(0)\right)^{2}-H\left(\frac{\omega}{x}\right)(0)^{2}\right\}=\frac{\pi}{2}\left(\omega_{x}^{2}(0)-u_{x x}^{2}(0)\right) .
\end{aligned}
$$

If $u_{x x}(0)=0$, the above equality is reduced to $\frac{\pi}{2} \omega_{x}^{2}(0)$.

Proof of (A.8). Applying (A.6) with $\left(u_{x}, \omega\right)$ replaced by $\left(u_{x x}, \omega_{x}\right)$ yields

$$
\begin{aligned}
\left\langle u_{x x} \omega_{x}, x\right\rangle & =\int \frac{\left(x \omega_{x}\right) H\left(x \omega_{x}\right)}{x} d x=\int \frac{\left(x \omega_{x}\right)\left(H\left(x \omega_{x}\right)-H\left(x \omega_{x}\right)(0)\right)}{x} d x \\
& =\frac{\pi}{2}\left(\left(x \omega_{x}\right)^{2}(0)+\left(x u_{x x}\right)^{2}(0)\right)=0
\end{aligned}
$$

where we have used $\left(x u_{x x}\right)(0)=\left(x \omega_{x}\right)(0)=0$ to obtain the last equality. 
Proof of (A.9). The first inequality in (A.9) is the standard Hardy inequality 14. Since $\omega$ is odd and $\omega_{x}(0)=0, \omega / x, \omega / x^{2} \in L^{2}(\mathbf{R})$. From (A.3), we have

$$
\frac{u_{x}-u_{x}(0)}{x}=H\left(\frac{\omega}{x}\right), \quad H\left(\frac{\omega}{x^{2}}\right)=\frac{1}{x}\left(H\left(\frac{\omega}{x}\right)-H\left(\frac{\omega}{x}\right)(0)\right) .
$$

Since $\omega$ is odd, we can simplify the second equality as follows

$$
H\left(\frac{\omega}{x}\right)(0)=-\frac{1}{\pi} \int \frac{\omega}{x^{2}} d x=0 \Rightarrow H\left(\frac{\omega}{x^{2}}\right)=\frac{1}{x} H\left(\frac{\omega}{x}\right)=\frac{1}{x} \frac{u_{x}-u_{x}(0)}{x}=\frac{u_{x}-u_{x}(0)}{x^{2}} .
$$

Using the isometry property of the Hilbert transform $H$ from $L^{2}(\mathbf{R})$ to $L^{2}(\mathbf{R})$, we conclude that

$$
\int \frac{\omega^{2}}{x^{2}} d x=\int \frac{\left(u_{x}-u_{x}(0)\right)^{2}}{x^{2}} d x, \quad \int \frac{\omega^{2}}{x^{4}} d x=\int \frac{\left(u_{x}-u_{x}(0)\right)^{2}}{x^{4}} d x .
$$

The following Lemma is an analogy of Lemma A.3 for Hölder continous functions. A.10, A.11) and (A.12) are from Córdoba \& Córdoba [6].

Lemma A.4 (Weighted estimate for $C^{\alpha}$ functions). Suppose that $u_{x}=H \omega$ and $\omega$ is odd in (A.10), A.12) and (A.13). (a) For $\beta \in(0,2)$, we have

$$
\begin{aligned}
& \int \frac{\left(u_{x}-u_{x}(0)\right)^{2}}{|x|^{1+\beta}} d x \leq \frac{1}{\min \left(\tan ^{2} \frac{\beta \pi}{4}, \cot ^{2} \frac{\beta \pi}{4}\right)} \int \frac{w^{2}}{|x|^{1+\beta}} d x \lesssim \frac{1}{(\beta \wedge(2-\beta))^{2}} \int \frac{w^{2}}{|x|^{1+\beta}} d x, \\
& \int \frac{u_{x}^{2}}{|x|^{1-\beta}} d x \leq \frac{1}{\min \left(\tan ^{2} \frac{\beta \pi}{4}, \cot ^{2} \frac{\beta \pi}{4}\right)} \int \frac{w^{2}}{|x|^{1-\beta}} d x \lesssim \frac{1}{(\beta \wedge(2-\beta))^{2}} \int \frac{w^{2}}{|x|^{1-\beta}} d x,
\end{aligned}
$$

provided that the right hand side is finite. Note that we do not need to assume that $\omega$ is odd in (A.11).

(b) For $\beta \in(0,2)$, we have

$$
\int \frac{\left(u_{x}-u_{x}(0)\right) \omega}{\operatorname{sgn}(x)|x|^{1+\beta}} d x \geq 0 .
$$

(c) $1 D$ Hardy inequality [14]: For $\beta \in(0,1)$, we have

$$
\int \frac{\left(u-u_{x}(0) x\right)^{2}}{|x|^{3+\beta}} d x \leq\left(\frac{2}{\beta+2}\right)^{2} \int \frac{\left(u_{x}-u_{x}(0)\right)^{2}}{|x|^{\beta+1}} d x \lesssim \frac{1}{\beta^{2}} \int \frac{\omega^{2}}{|x|^{\beta+1}} .
$$

The first inequality in (A.13) is the Hardy inequality [14] and the second inequality in A.13) follows from (A.10).

A.2. Estimate of the $C^{\alpha}$ approximate self-similar solution. We establish the estimate of the approximate self-similar solution in Lemma 4.3 in this section.

Proof of Lemma 4.3.

Proof of (4.10). Recall the explicit formula for $\omega_{\alpha}, u_{\alpha, x}$ in (4.2) and the weight $\varphi_{\alpha}, \psi_{\alpha}$ in (4.4). Denote $c_{\alpha}=\cos (\alpha \pi / 2), s_{\alpha}=\sin (\alpha \pi / 2)$. Without loss of generality, we consider $x>0$. We have

$$
\varphi_{\alpha}=\frac{1}{2 s_{\alpha}} \frac{\left(1+2 c_{\alpha} x^{\alpha}+x^{2 \alpha}\right)^{2}}{x^{1+3 \alpha}}, \quad \frac{x \varphi_{\alpha, x}}{\varphi_{\alpha}}=x\left(\log \left(\varphi_{\alpha}\right)_{x}=\frac{2\left(2 \alpha x^{2 \alpha}+2 \alpha c_{\alpha} x^{\alpha}\right)}{1+2 c_{\alpha} x^{\alpha}+x^{2 \alpha}}-(1+3 \alpha),\right.
$$

which implies

$$
\begin{aligned}
& \frac{1}{2 \varphi_{\alpha}}\left(\frac{1}{\alpha} x \varphi_{\alpha}\right)_{x}+\left(\bar{c}_{\omega}+u_{\alpha, x}\right)=\frac{1}{2 \alpha}+\frac{x \varphi_{\alpha, x}}{2 \alpha \varphi_{\alpha}}-1+\frac{2\left(1+c_{\alpha} x^{\alpha}\right)}{1+2 c_{\alpha} x^{\alpha}+x^{2 \alpha}} \\
= & -\frac{1+3 \alpha}{2 \alpha}+\frac{2\left(x^{2 \alpha}+c_{\alpha} x^{\alpha}\right)}{1+2 c_{\alpha} x^{\alpha}+x^{2 \alpha}}+\frac{1}{2 \alpha}-1+\frac{2\left(1+c_{\alpha} x^{\alpha}\right)}{1+2 c_{\alpha} x^{\alpha}+x^{2 \alpha}}=-\frac{3}{2}-1+2=-\frac{1}{2},
\end{aligned}
$$


ON THE FINITE TIME BLOWUP OF THE DE GREGORIO MODEL FOR THE 3D EULER EQUATION 45

which is the first identity in (4.10). The second identity can be proved similarly. For the third inequality, we have

$$
\begin{aligned}
& u_{\alpha, x x} \psi_{\alpha}=\partial_{x}\left(\frac{2\left(1+c_{\alpha} x^{\alpha}\right)}{1+2 c_{\alpha} x^{\alpha}+x^{2 \alpha}}\right) \frac{\left(1+2 c_{\alpha} x^{\alpha}+x^{2 \alpha}\right)^{2}}{2 s_{\alpha} \alpha^{2}} x^{1-3 \alpha} \\
= & \left(2 \alpha c_{\alpha} x^{\alpha-1}\left(1+2 c_{\alpha} x^{\alpha}+x^{2 \alpha}\right)-2\left(1+c_{\alpha} x^{\alpha}\right) 2 \alpha\left(c_{\alpha} x^{\alpha-1}+x^{2 \alpha-1}\right)\right) x^{1-3 \alpha}\left(2 s_{\alpha} \alpha^{2}\right)^{-1} \\
= & 2 \alpha\left(-c_{\alpha} x^{3 \alpha-1}-2 x^{2 \alpha-1}-c_{\alpha} x^{\alpha-1}\right) x^{1-3 \alpha}\left(2 s_{\alpha} \alpha^{2}\right)^{-1}=-\left(2 x^{-\alpha}+c_{\alpha}\left(x^{-2 \alpha}+1\right)\right)\left(s_{\alpha} \alpha\right)^{-1},
\end{aligned}
$$

which is monotone increasing with respect to $x$ and the desired inequality follows.

Proof of (4.11). Since all quantities are symmetric, we only consider $x \geq 0$. For (4.11), we have

$$
\begin{aligned}
\left|\frac{x \omega_{\alpha, x}}{\omega}\right| & =\alpha\left|\frac{1-x^{\alpha}}{1+2 \cos \left(\frac{\alpha \pi}{2}\right)|x|^{\alpha}+|x|^{2 \alpha}}\right| \leq \alpha, \\
\left|\frac{x^{2} \omega_{\alpha, x x}+x \omega_{\alpha, x}}{\omega_{\alpha}}\right| & =\alpha^{2}\left|\frac{1-6 x^{2 \alpha}+x^{4 \alpha}-2 \cos \left(\frac{\alpha \pi}{2}\right) x^{\alpha}\left(1+x^{2 \alpha}\right)}{\left(1+2 \cos \left(\frac{\alpha \pi}{2}\right) x^{\alpha}+x^{2 \alpha}\right)^{2}}\right| \lesssim \alpha^{2},
\end{aligned}
$$

uniformly for all $x \geq 0$. Using the triangle inequality, we get

$$
\left\|\frac{x^{2} \omega_{\alpha, x x}}{\omega_{\alpha}}\right\|_{\infty} \leq\left\|\frac{x \omega_{\alpha, x}}{\omega}\right\|_{\infty}+\left\|\frac{x^{2} \omega_{\alpha, x x}+x \omega_{\alpha, x}}{\omega_{\alpha}}\right\|_{\infty} \lesssim \alpha .
$$

Proof of (4.12). Assume $y \geq 0$. For (4.12), we have

$$
\begin{aligned}
\left|\frac{u_{\alpha}(y)}{y}-u_{\alpha, x}(0)\right| & =\left|\frac{1}{y} \int_{0}^{y}\left(\frac{2\left(1+\cos \left(\frac{\alpha \pi}{2}\right)|x|^{\alpha}\right)}{1+2 \cos \left(\frac{\alpha \pi}{2}\right)|x|^{\alpha}+|x|^{2 \alpha}}-2\right) d x\right|=\left|\frac{1}{y} \int_{0}^{y} \frac{2|x|^{2 \alpha}+2 \cos \left(\frac{\alpha \pi}{2}\right)|x|^{\alpha}}{1+\cos \left(\frac{\alpha \pi}{2}\right)|x|^{\alpha}+|x|^{2 \alpha}} d x\right| \\
& \left.\left.\lesssim\left|\frac{1}{y} \int_{0}^{y}\right| x\right|^{\alpha} \wedge 1 d x\left|\leq \min \left(\left|\frac{1}{y} \frac{|y|^{1+\alpha}}{1+\alpha}\right|, 1\right) \lesssim\right| y\right|^{\alpha} \wedge 1 \\
\left|\frac{u_{\alpha}(y)}{y}-u_{\alpha, x}(y)\right| & =\left|\frac{1}{y} \int_{0}^{y}\left(u_{\alpha, x}(x)-u_{\alpha, x}(y)\right) d x\right|=\left|\frac{1}{y} \int_{0}^{y} x u_{\alpha, x x}(x) d x\right| \\
& =\left.\left.\left|\frac{2 \alpha}{y} \int_{0}^{y} \frac{x^{\alpha}\left(2 x^{\alpha}+\cos \left(\frac{\alpha \pi}{2}\right)\left(1+x^{2 \alpha}\right)\right)}{\left(1+\cos \left(\frac{\alpha \pi}{2}\right)|x|^{\alpha}+|x|^{2 \alpha}\right)^{2}} d x\right| \lesssim\left|\frac{2 \alpha}{y} \int_{0}^{y}\right| x\right|^{\alpha} \wedge 1 d x|\lesssim \alpha| y\right|^{\alpha} \wedge 1 .
\end{aligned}
$$

Proof of (4.13). For (4.13), the first two inequalities follow from the definition of $\varphi_{\alpha}, \psi_{\alpha}$ in (4.4) and

$$
-\frac{1}{\omega_{\alpha}} \asymp \frac{1}{\alpha}\left(|x|^{\alpha}+|x|^{-\alpha}\right) .
$$

From the definition $\psi_{\alpha}=x^{2} \varphi_{\alpha} / \alpha^{2}$, we know

$$
\frac{x \psi_{\alpha, x}}{\psi_{\alpha}}-1=\frac{x\left(x^{2} \varphi_{\alpha}\right)_{x}}{x^{2} \varphi_{\alpha}}-1=\frac{x^{3} \varphi_{\alpha, x}+2 x^{2} \varphi_{\alpha}}{x^{2} \varphi_{\alpha}}-1=\frac{x \varphi_{\alpha, x}}{\varphi_{\alpha}}+1 .
$$

Hence, for the third inequality in (4.13), we get

$$
\left|\frac{x \psi_{\alpha, x}}{\psi_{\alpha}}-1\right|=\left|\frac{x \varphi_{\alpha, x}}{\varphi_{\alpha}}+1\right|=\alpha\left|\frac{-3+x^{2 \alpha}-2 \cos \left(\frac{\alpha \pi}{2}\right) x^{\alpha}}{1+\cos \left(\frac{\alpha \pi}{2}\right) x^{\alpha}+x^{2 \alpha}}\right| \lesssim \alpha .
$$

Proof of (4.14). Recall the definition of error term in (4.7)

$$
F_{\alpha}\left(\omega_{\alpha}\right)=-a\left(u_{\alpha}-u_{\alpha, x}(0) x\right) \omega_{\alpha, x} .
$$


For the first inequality in (4.14), we use the result (4.11) and (4.12) that we just proved to yield

$$
\begin{aligned}
\left\langle F_{\alpha}\left(\omega_{\alpha}\right)^{2}, \varphi_{\alpha}\right\rangle & =a^{2}\left\langle\left(u_{\alpha}-u_{\alpha, x}(0) x\right)^{2} \omega_{\alpha, x}^{2},-\frac{1}{\operatorname{sgn}(x) \omega_{\alpha}} \frac{1+2 \cos \left(\frac{\alpha \pi}{2}\right)|x|^{\alpha}+|x|^{2 \alpha}}{|x|^{1+2 \alpha}}\right\rangle \\
& \lesssim a^{2}\left\langle\left(u_{\alpha}-u_{\alpha, x}(0) x\right)^{2} \omega_{\alpha, x}^{2},-\frac{1}{\operatorname{sgn}(x) \omega_{\alpha}}\left(|x|^{-1}+|x|^{-1-2 \alpha}\right)\right\rangle \\
& =a^{2}\left\langle\left(\frac{u_{\alpha}}{x}-u_{\alpha, x}(0)\right)^{2} \frac{x^{2} \omega_{\alpha, x}^{2}}{\omega_{\alpha}^{2}},\left|\omega_{\alpha}\right|\left(|x|^{-1}+|x|^{-1-2 \alpha}\right)\right\rangle \\
& \lesssim a^{2}\left\langle\left(|x|^{\alpha} \wedge 1\right)^{2} \alpha^{2},\left|\omega_{\alpha}\right|\left(|x|^{-1}+|x|^{-1-2 \alpha}\right)\right\rangle \\
& \lesssim a^{2} \alpha^{2}\left\langle\left|\omega_{\alpha}\right|,|x|^{-1}\right\rangle=a^{2} \alpha^{2}\left|\int_{\mathbf{R}} \frac{\omega_{\alpha}}{x} d x\right|=a^{2} \alpha^{2} \pi\left|u_{\alpha, x}(0)\right| \lesssim a^{2} \alpha^{2}
\end{aligned}
$$

where we have used $\omega_{\alpha} / x \leq 0$ for all $x \in \mathbf{R}$ to obtain the last line.

For the second inequality in (4.7), we first rewrite $\left(F_{\alpha}\left(\omega_{\alpha}\right)\right)_{x}$ as follows

$$
\begin{aligned}
\left(F_{\alpha}\left(\omega_{\alpha}\right)\right)_{x} & =-a\left(\left(u_{\alpha}-u_{\alpha, x}(0) x\right) \omega_{\alpha, x}\right)_{x}=-a\left\{\left(\frac{u_{\alpha}}{x}-u_{\alpha, x}(0)\right)\left(x \omega_{\alpha, x}\right)\right\}_{x} \\
& =-a\left(\frac{u_{\alpha}}{x}-u_{\alpha, x}(0)\right)_{x}\left(x \omega_{\alpha, x}\right)-a\left(\frac{u_{\alpha}}{x}-u_{\alpha, x}(0)\right)\left(x \omega_{\alpha, x}\right)_{x} \\
& =a\left(\frac{u_{\alpha}(x)}{x}-u_{\alpha, x}(x)\right) \omega_{\alpha, x}-a\left(\frac{u_{\alpha}}{x}-u_{\alpha, x}(0)\right) \frac{x^{2} \omega_{\alpha, x x}+x \omega_{\alpha, x}}{x} .
\end{aligned}
$$

We can use (4.12) to estimate $u_{\alpha}$ and $u_{\alpha, x}$ as follows:

$$
\left|\left(F_{\alpha}\left(\omega_{\alpha}\right)\right)_{x}\right| \lesssim a \alpha\left(|x|^{\alpha} \wedge 1\right)\left|\omega_{\alpha, x}\right|+a\left(|x|^{\alpha} \wedge 1\right)\left|\frac{x^{2} \omega_{\alpha, x x}+x \omega_{\alpha, x}}{x}\right| .
$$

Then we use (4.11) to estimate $\omega_{\alpha, x}, x^{2} \omega_{\alpha, x x}+x \omega_{\alpha, x}$

$$
\left|\left(F_{\alpha}\left(\omega_{\alpha}\right)\right)_{x}\right| \lesssim a \alpha\left(|x|^{\alpha} \wedge 1\right) \alpha\left|\frac{\omega_{\alpha}}{x}\right|+a\left(|x|^{\alpha} \wedge 1\right) \alpha^{2}\left|\frac{\omega_{\alpha}}{x}\right| \lesssim a \alpha^{2}\left(|x|^{\alpha} \wedge 1\right)\left|\frac{\omega_{\alpha}}{x}\right| .
$$

Hence, we can estimate the weighted $H^{1}$ error as follows

$$
\begin{aligned}
& \left\langle\left(F_{\alpha}\left(\omega_{\alpha}\right)\right)_{x}^{2}, \psi\right\rangle=\left\langle\left(F_{\alpha}\left(\omega_{\alpha}\right)\right)_{x}^{2}, \frac{x^{2}}{\alpha^{2}\left|\omega_{\alpha}\right|} \frac{1+2 \cos \left(\frac{\alpha \pi}{2}\right)|x|^{\alpha}+|x|^{2 \alpha}}{|x|^{1+2 \alpha}}\right\rangle \\
\lesssim & \left\langle\left(F_{\alpha}\left(\omega_{\alpha}\right)\right)_{x}^{2}, \frac{x^{2}}{\alpha^{2}\left|\omega_{\alpha}\right|}\left(|x|^{-1}+|x|^{-1-2 \alpha}\right)\right\rangle \\
\lesssim & a^{2} \alpha^{4}\left\langle\left(|x|^{\alpha} \wedge 1\right)^{2}\left|\frac{\omega_{\alpha}}{x}\right|^{2}, \frac{x^{2}}{\alpha^{2}\left|\omega_{\alpha}\right|}\left(|x|^{-1}+|x|^{-1-2 \alpha}\right)\right\rangle \\
\lesssim & a^{2} \alpha^{4}\left\langle\left|\frac{\omega_{\alpha}}{x}\right|^{2}, \frac{x^{2}}{\alpha^{2}\left|\omega_{\alpha}\right|}|x|^{-1}\right\rangle=a^{2} \alpha^{2}\left\langle\left|\omega_{\alpha}\right|,|x|^{-1}\right\rangle=a^{2} \alpha^{2} \pi\left|u_{\alpha, x}(0)\right| \lesssim a^{2} \alpha^{2} .
\end{aligned}
$$

Proof of (4.15). Using (4.11), we get

$$
\left(|x|^{\alpha} \wedge 1\right)\left|\omega_{\alpha, x}\right| \lesssim \alpha\left(|x|^{\alpha} \wedge 1\right)\left|\frac{\omega_{\alpha}}{x}\right| .
$$

Note that the above bound is the same as (A.14) up to a factor $a \alpha$. Hence, we can use the same estimate as in (A.15) to yield

$$
\left\langle\left(|x|^{\alpha} \wedge 1\right)^{2} \omega_{\alpha, x}^{2}, \psi_{\alpha}\right\rangle \lesssim 1
$$

Proof of Lemma 4.5 . 
ON THE FINITE TIME BLOWUP OF THE DE GREGORIO MODEL FOR THE 3D EULER EQUATION 47

Proof of (4.16). Firstly, we apply the weighted inequality (A.11) with $\beta=\alpha, 2-\alpha$ to yield

$$
\int \frac{u_{x}^{2}}{|x|^{1-\alpha}} d x \lesssim \frac{1}{\alpha^{2}} \int \frac{\omega^{2}}{|x|^{1-\alpha}} d x, \quad \int u_{x x}^{2}|x|^{1-\alpha} d x \lesssim \frac{1}{\alpha^{2}} \int \omega_{x}^{2}|x|^{1-\alpha} d x
$$

It follows that

$$
\begin{aligned}
\left\|u_{x}\right\|_{\infty}^{2} & \leq 2 \int\left|u_{x} u_{x x}\right| d x \leq 2\left(\int \frac{u_{x}^{2}}{|x|^{1-\alpha}} d x\right)^{1 / 2}\left(\int u_{x x}^{2}|x|^{1-\alpha} d x\right)^{1 / 2} \\
& \lesssim \frac{1}{\alpha^{2}}\left(\int \frac{\omega^{2}}{|x|^{1-\alpha}} d x\right)^{1 / 2}\left(\int \omega_{x}^{2}|x|^{1-\alpha} d x\right)^{1 / 2} .
\end{aligned}
$$

Using the asymptotic properties of $\varphi_{\alpha}, \psi_{\alpha}$ in (4.13) and $|x|^{1-\alpha} \leq|x|^{1-3 \alpha}+|x|^{1+\alpha}$, we conclude

$$
\left\|u_{x}\right\|_{\infty} \lesssim\left\langle\omega^{2}, \varphi_{\alpha}\right\rangle^{1 / 4}\left\langle\omega_{x}^{2}, \psi_{\alpha}\right\rangle^{1 / 4}
$$

Proof of (4.17). Firstly, we use $\omega(0)=0$ and integration by parts to get

$$
J \triangleq \int_{0}^{\infty} \omega_{x}(y)\left(\frac{y}{x} \log \left|\frac{x+y}{x-y}\right|-2\right) d y=-P . V . \int_{0}^{+\infty} \omega(y)\left(\frac{1}{x} \log \left|\frac{x+y}{x-y}\right|+\frac{y}{x}\left(\frac{1}{y+x}-\frac{1}{y-x}\right)\right) d y .
$$

Using

$$
\frac{y}{x}\left(\frac{1}{y+x}-\frac{1}{y-x}\right)=\frac{1}{x-y}-\frac{1}{x+y}
$$

$\tilde{u}=u-u_{x}(0) x$ and that $\omega$ is odd, we derive

$$
J=-P . V . \int_{0}^{+\infty} \omega(y)\left(\frac{1}{x} \log \left|\frac{x+y}{x-y}\right|+\left(\frac{1}{x-y}-\frac{1}{x+y}\right)\right) d y=\pi\left(\frac{u}{x}-u_{x}\right)=\pi\left(\frac{\tilde{u}}{x}-\tilde{u}_{x}\right) .
$$

Using this integral formula of $\tilde{u} / x-\tilde{u}_{x}=J$, the asymptotic property (4.13) and the CauchySchwartz inequality, we have

$$
\begin{aligned}
\left|\frac{\tilde{u}}{x}-\tilde{u}_{x}\right| & \lesssim\left\langle\omega_{x}^{2},|y|^{1-3 \alpha}+|y|^{1+\alpha}\right\rangle^{1 / 2}\left(\int_{0}^{\infty}\left(\frac{y}{x} \log \left|\frac{x+y}{x-y}\right|-2\right)^{2} \frac{1}{|y|^{1-3 \alpha}+|y|^{1+\alpha}} d y\right)^{1 / 2} \\
& \lesssim \alpha^{3 / 2}\left\langle\omega_{x}^{2}, \psi_{\alpha}\right\rangle\left(\int_{0}^{\infty}\left(\frac{y}{x} \log \left|\frac{1+y / x}{1-y / x}\right|-2\right)^{2} \frac{1}{|y|^{1-3 \alpha}+|y|^{1+\alpha}} d y\right)^{1 / 2} .
\end{aligned}
$$

Next, we estimate the integral

$$
I(x) \triangleq \int_{0}^{\infty}\left(\frac{y}{x} \log \left|\frac{1+y / x}{1-y / x}\right|-2\right)^{2} \frac{1}{|y|^{1-3 \alpha}+|y|^{1+\alpha}} d y .
$$

To conclude (4.17), it suffices to prove

$$
|I(x)| \lesssim \frac{1}{\alpha}\left(|x|^{2 \alpha} \wedge 1\right)
$$

Without loss of generality, we assume $x \geq 0$. Using change of variable $s=y / x$ yields

$$
\begin{aligned}
I(x) & =\int_{0}^{\infty}\left(s \log \left|\frac{s+1}{s-1}\right|-2\right)^{2} \frac{x}{(x s)^{1-3 \alpha}+(x s)^{1+\alpha}} d s \\
& =\left(\int_{0}^{x^{-1} \wedge 1 / 2}+\int_{x^{-1} \wedge 1 / 2}^{1 / 2}+\int_{1 / 2}^{\infty}\right)\left(s \log \left|\frac{s+1}{s-1}\right|-2\right)^{2} \frac{x}{(x s)^{1-3 \alpha}+(x s)^{1+\alpha}} d s \\
& \triangleq I_{1}(x)+I_{2}(x)+I_{3}(x) .
\end{aligned}
$$

We introduce $f(s)$ and it satisfies the following estimate

$$
f(s) \triangleq|s \log | \frac{s+1}{s-1}|-2| \lesssim s^{-1}, \forall s>2 ; \quad|s \log | \frac{s+1}{s-1}|-2| \lesssim 1, \forall s<1 / 2 .
$$


For $I_{3}(x)$, we use $(x s)^{1-3 \alpha}+(x s)^{1-\alpha} \geq x s$ and the decay of $f(s)$ in (A.16) to obtain

$$
\begin{aligned}
I_{3}(x) & \leq \min \left\{\int_{1 / 2}^{\infty}\left(s \log \left|\frac{s+1}{s-1}\right|-2\right)^{2} \frac{x}{x s} d s, \int_{1 / 2}^{\infty}\left(s \log \left|\frac{s+1}{s-1}\right|-2\right)^{2} \frac{x}{(x s)^{1-3 \alpha}} d s\right\} \\
& =\min \left\{\int_{1 / 2}^{\infty} f(s)^{2} \frac{1}{s} d s,|x|^{3 \alpha} \int_{1 / 2}^{\infty} f(s)^{2} \frac{1}{s^{1-3 \alpha}} d s\right\} \lesssim \min \left(1,|x|^{3 \alpha}\right) .
\end{aligned}
$$

Next, we estimate $I_{1}(x)$. For $s \in[0,1 / 2]$, we use the boundedness of $f(s)$ in (A.16) to obtain

$$
\begin{aligned}
I_{1}(x) & \lesssim \int_{0}^{x^{-1} \wedge 1 / 2} \frac{x}{(x s)^{1-3 \alpha}+(x s)^{1+\alpha}} d s \leq \int_{0}^{x^{-1} \wedge 1 / 2} \frac{x}{(x s)^{1-3 \alpha}} d s \\
& =x^{3 \alpha} \int_{0}^{x^{-1} \wedge 1 / 2} s^{-1+3 \alpha} d s=\frac{1}{3 \alpha} x^{3 \alpha}\left(x^{-1} \wedge 1 / 2\right)^{3 \alpha} \lesssim \frac{1}{\alpha}|x|^{2 \alpha} \wedge 1 .
\end{aligned}
$$

For $I_{2}(x)$, by definition, $I_{2}(x)=0$ if $x^{-1} \geq 1 / 2$ (i.e. $x \leq 2$ ). For $x>2$, we have

$$
\begin{aligned}
I_{2}(x) & \lesssim \int_{x^{-1}}^{1 / 2} \frac{x}{(x s)^{1-3 \alpha}+(x s)^{1+\alpha}} d s \leq \int_{x^{-1}}^{1 / 2} \frac{x}{(x s)^{1+\alpha}} d s \\
& =x^{-\alpha} \int_{x^{-1}}^{1 / 2} s^{-1-\alpha} d s \leq x^{-\alpha} \frac{1}{\alpha}\left(x^{-1}\right)^{-\alpha} \leq \frac{1}{\alpha} \leq \frac{1}{\alpha}|x|^{2 \alpha} \wedge 1 .
\end{aligned}
$$

Therefore, we conclude

$$
I(x)=I_{1}(x)+I_{2}(x)+I_{3}(x) \lesssim \frac{1}{\alpha}\left(|x|^{2 \alpha} \wedge 1\right) .
$$

This completes the proof of (4.17).

Proof of (4.18). Without loss of generality, we assume $x \geq 0$. Using (4.13) and the CauchySchwartz inequality, we get

$$
\begin{aligned}
|\omega(x)| & \leq \int_{0}^{x}\left|\omega_{x}\right| d x \leq\left\langle\omega_{x}^{2},|x|^{1-3 \alpha}+|x|^{1+\alpha}\right\rangle^{1 / 2}\left(\int_{0}^{x} \frac{1}{|x|^{1-3 \alpha}+|x|^{1+\alpha}} d x\right)^{1 / 2} \\
& \lesssim \alpha^{3 / 2}\left\langle\omega_{x}^{2}, \psi\right\rangle^{1 / 2}\left(\int_{0}^{x} \frac{1}{|x|^{1-3 \alpha}+|x|^{1+\alpha}} d x\right)^{1 / 2} .
\end{aligned}
$$

If $x \in[0,1]$, the integral is bounded by

$$
\int_{0}^{x}|x|^{-1+3 \alpha} d x \lesssim \frac{1}{\alpha}|x|^{3 \alpha} \leq \frac{1}{\alpha}|x|^{2 \alpha} \leq \frac{1}{\alpha}\left(|x|^{2 \alpha} \wedge 1\right) .
$$

Otherwise, it is bounded by

$$
\int_{0}^{1}|x|^{-1+3 \alpha} d x+\int_{1}^{x}|x|^{-1-\alpha} d x \lesssim \frac{1}{\alpha} \leq \frac{1}{\alpha}\left(|x|^{2 \alpha} \wedge 1\right) .
$$

Therefore, combining (A.17), (A.18) and (A.19), we conclude

$$
|\omega(x)| \lesssim \alpha^{3 / 2}\left\langle\omega_{x}^{2}, \psi\right\rangle^{1 / 2} \alpha^{-1 / 2}\left(|x|^{2 \alpha} \wedge 1\right)^{1 / 2}=\alpha\left\langle\omega_{x}^{2}, \psi\right\rangle^{1 / 2}\left(|x|^{\alpha} \wedge 1\right) .
$$

\section{A.3. Proof of other Lemmas.}

Proof of Lemma 4.10. Recall the definition of $F(s, \beta)$

$$
F(s, \beta) \triangleq \frac{1-s^{1+\beta}}{1+s^{1+\beta}} \frac{2 s}{1-s^{2}}\left(\log \left|\frac{s+1}{s-1}\right|\right)^{-1}, \quad s \in[0,1], \beta \in[1,2] .
$$

For $s \in[0,1], s^{1+\beta}$ is decreasing with respect to $\beta$. Hence

$$
\frac{1-s^{1+\beta}}{1+s^{1+\beta}} \text { is increasing w.r.t. } \beta \Rightarrow F(s, \beta) \text { is increasing w.r.t. } \beta \text {. }
$$


ON THE FINITE TIME BLOWUP OF THE DE GREGORIO MODEL FOR THE 3D EULER EQUATION 49

Next, we show that $F(s, 1) \leq 1$. Denote

$$
G(s)=\log \left|\frac{s+1}{s-1}\right|-\frac{2 s}{1+s^{2}}, \quad s \in[0,1] .
$$

Note that

$$
G^{\prime}(s)=\frac{2}{1-s^{2}}-\frac{2}{1+s^{2}}+\frac{4 s^{2}}{\left(1+s^{2}\right)^{2}} \geq 0, \quad \lim _{s \rightarrow 0^{+}} G(s)=0 .
$$

We conclude $G(s) \geq 0$ for $s \in[0,1]$. It follows that

$$
F(s, 1)=\frac{1-s^{2}}{1+s^{2}} \frac{2 s}{1-s^{2}}\left(\log \left|\frac{s+1}{s-1}\right|\right)^{-1}=\frac{2 s}{1+s^{2}}\left(\log \left|\frac{s+1}{s-1}\right|\right)^{-1} \leq 1 .
$$

The equality is achieved at $s=0$. Next, we verify that $F(s, \beta) \leq 1+0.015(\beta-1)$. We split $s, \beta \in[0,1] \times[1,2]$ into several domains and prove this inequality separately.

Case a. $(s, \beta) \in[0.5,1] \times[1,2]$. Using the fact that $F(s, \beta)$ is increasing w.r.t. $\beta$, we know

$$
F(s, \beta) \leq F(s, 2)<0.96<1 \quad \forall s \geq 0.5, \beta \in[1,2],
$$

where the inequality $F(s, 2)<1$ for $s \in[0.5,1]$ can be verified numerically with rigorous error control. For $s$ close to $1, F(s, \beta)$ goes to 0 since $\log ((1+s) /(1-s))$ blows up at $s=1$.

Case b. $(s, \beta) \in[0.04,0.5] \times[1,2]$. In this domain, $F(s, \beta)$ is smooth. Denote

$$
M(\beta) \triangleq \max _{s \in[0.04,0.5]} F(s, \beta) .
$$

For $\beta=2$, we can estimate $F(s, \beta)$ using Matlab (evaluating the function at some discrete points with error control and then using the boundedness of $\partial_{s} F(s, \beta)$ to estimate other points)

$$
\begin{aligned}
M(2) & =\max _{s \in[0.04,0.5]} F(s, 2) \leq 1.0123, \\
\Rightarrow M(\beta)-1 & \leq M(2)-1 \leq 0.0123 \leq 0.015(\beta-1) \quad \forall \beta \in[1.85,2] .
\end{aligned}
$$

The constant 0.015 in (4.66) comes from the above inequality $M(2) \leq 1.0123$. Similarly, we can estimate $M(1.85), M(1.5), M(1.1)$ to get

$$
\begin{aligned}
M(1.85) & \leq 1.0071 \Rightarrow M(\beta)-1 \leq M(1.85)-1 \leq 0.0071 \leq 0.015(\beta-1), \forall \beta \in[1.5,1.85], \\
M(1.5) & \leq 1.0007 \Rightarrow M(\beta)-1 \leq 0.0007 \leq 0.015(\beta-1), \quad \forall \beta \in[1.1,1.5], \\
M(1.1) \leq 0.9989 & \Rightarrow M(\beta)-1 \leq 0 \leq 0.015(\beta-1), \quad \forall \beta \in[1,1.1] .
\end{aligned}
$$

Therefore, for $s, \beta \in[0.04,0.5] \times[1,2]$, the above inequalities imply $F(s, \beta) \leq M(\beta) \leq 1+$ $0.015(\beta-1)$.

Case c. $(s, \beta) \in[0,0.04] \times[1,2]$. The partial derivative $\partial_{\beta} F(s, \beta)$ is given by

$$
\partial_{\beta} F(s, \beta)=-\frac{2 s^{1+\beta} \log s}{\left(1+s^{1+\beta}\right)^{2}} \frac{2 s}{1-s^{2}}\left(\log \left|\frac{s+1}{s-1}\right|\right)^{-1} .
$$

It is not singular near $s=0$ due to the power $s^{2+\beta}$. Since $s^{1+\beta}$ is decreasing with respect to $\beta$ and $t /(1+t)^{2}$ is increasing for $t \in[0,1]$, we get

$$
\frac{s^{1+\beta}}{\left(1+s^{1+\beta}\right)^{2}} \leq \frac{s^{2}}{\left(1+s^{2}\right)^{2}} .
$$

Notice that $-\log (s) \geq 0$ for $s \in[0,1]$. We obtain

$$
0 \leq \partial_{\beta} F(s, \beta) \leq \frac{-2 s^{2} \log s}{\left(1+s^{2}\right)^{2}} \frac{2 s}{1-s^{2}}\left(\log \left|\frac{s+1}{s-1}\right|\right)^{-1} \triangleq H(s) .
$$

For $s \in[0,0.04]$, we can estimate $H(s)$ numerically with rigorous error control and obtain $H(s) \leq 0.011$. Therefore for $s \in[0,0.04]$, we yield

$$
F(s, \beta) \leq F(s, 1)+0.011(\beta-1) \leq 1+0.015(\beta-1) .
$$

Combining case (a), (b) and (c), we conclude the proof of (4.66) and Lemma 4.10 
Proof of Lemma 4.13. Assume that $S^{1}$ is $\pi$ - periodic. Then we have

$$
u_{x}(x)=\frac{1}{\pi} \int_{0}^{\pi} \omega(y) \cot (x-y) d y, \quad u(x)=-\frac{1}{\pi} \int_{0}^{\pi / 2} \log \left|\frac{\tan y+\tan x}{\tan y-\tan x}\right| \omega(y) d y .
$$

Symmetrizing the convolution kernel as we did in the proof of Lemma 4.9 and (4.65), we obtain

$$
I \triangleq \frac{1}{\pi} \int_{0}^{\pi / 2}\left(u_{x} \omega-a u \omega_{x}\right)(\cot x)^{\beta} d x=\frac{1}{\pi} \int_{0}^{\pi} \omega(x) \omega(y) K_{\text {sym }}(x, y) d x d y,
$$

where the symmetrized kernel $K_{\text {sym }}$ is given by

$$
\begin{aligned}
K_{\text {sym }}(x, y) & =\frac{1+a}{2}(\cot y)^{\beta-1}\left(\tau\left(s^{\beta-1}+1\right) \log \left|\frac{s+1}{s-1}\right|-\left(s^{\beta-1}-1\right) \frac{2 s}{s^{2}-1}\right) \\
& +\frac{1+a}{2}(\cot y)^{\beta+1}\left(\tau\left(s^{\beta+1}+1\right) \log \left|\frac{s+1}{s-1}\right|-\left(s^{\beta+1}-1\right) \frac{2 s}{s^{2}-1}\right),
\end{aligned}
$$

with

$$
\tau=\frac{a \beta}{1+a}, \quad s=\frac{\tan y}{\tan x}=\frac{\cot x}{\cot y} .
$$

Since $a, \beta$ satisfy the assumption in Lemma 4.9, from the proof of Lemma 4.9, we know that

$$
\tau\left(s^{\beta+1}+1\right) \log \left|\frac{s+1}{s-1}\right|-\left(s^{\beta+1}-1\right) \frac{2 s}{s^{2}-1} \geq 0, \quad \forall s \geq 0 .
$$

Recall $\beta>1$. For $s \in[0,1]$, we have $s^{\beta-1} \geq s^{\beta+1}$ and

$$
\frac{s^{\beta-1}+1}{1-s^{\beta-1}}\left(1-s^{2}\right) \geq \frac{s^{\beta+1}+1}{1-s^{\beta+1}}\left(1-s^{2}\right) .
$$

It is not difficult to show that the above inequality also holds true for $s \geq 1$ if we replace $s$ by $s^{-1}$. Combining (A.22) and (A.23), we get

$$
\frac{s^{\beta-1}+1}{1-s^{\beta-1}}\left(1-s^{2}\right) \geq \frac{s^{\beta+1}+1}{1-s^{\beta+1}}\left(1-s^{2}\right) \geq \frac{1}{\tau} 2 s\left(\log \left|\frac{s+1}{s-1}\right|\right)^{-1},
$$

which implies

$$
\tau\left(s^{\beta-1}+1\right) \log \left|\frac{s+1}{s-1}\right|-\left(s^{\beta-1}-1\right) \frac{2 s}{s^{2}-1} \geq 0, \quad \forall s \geq 0 .
$$

Substituting (A.22) and (A.24) in (A.21), we conclude

$$
K_{\text {sym }}(x, y) \geq 0, \quad \forall x, y \in[0, \pi / 2] .
$$

Finally, noticing that $\omega(x) \omega(y) \geq 0$ for all $x, y \in[0, \pi / 2]$, we prove $I \geq 0$ in A.20.

Acknowledgments. The authors would like to acknowledge the generous support from the National Science Foundation under Grant No. DMS-1613861.

\section{REFERENCES}

[1] A Castro and D Córdoba. Infinite energy solutions of the surface quasi-geostrophic equation. Advances in Mathematics, 225(4):1820-1829, 2010

[2] J Chen, T. Y. Hou, and D Huang. Supplementary materials for the paper "on the finite time blowup of the de gregorio model for the 3d euler equations". preprint, 2019.

[3] K Choi, TY Hou, A Kiselev, G Luo, V Sverak, and Y Yao. On the finite-time blowup of a 1D model for the 3D axisymmetric Euler equations. CPAM, 70(11):2218-2243, 2017.

[4] P Constantin. On the Euler equations of incompressible fluids. Bulletin of the American Mathematical Society, 44(4):603-621, 2007.

[5] P Constantin, P. D. Lax, and A. Majda. A simple onedimensional model for the threedimensional vorticity equation. CPAM, 38(6):715-724, 1985.

[6] A Córdoba, D Córdoba, and M. A. Fontelos. Integral inequalities for the hilbert transform applied to a nonlocal transport equation. Journal de Mathématiques Pures et Appliquées, 88(6):529-540, 2006.

[7] S De Gregorio. On a one-dimensional model for the three-dimensional vorticity equation. Journal of Statistical Physics, 59(5-6):1251-1263, 1990.

[8] S De Gregorio. A partial differential equation arising in a $1 \mathrm{~d}$ model for the $3 \mathrm{~d}$ vorticity equation. Mathematical Methods in the Applied Sciences, 19(15):1233-1255, 1996. 
[9] T. M. Elgindi, T. E. Ghoul, and N. Masmoudi. Stable self-similar blowup for a family of nonlocal transport equations. In preparation, 2019.

[10] T. M. Elgindi and I. J. Jeong. On the effects of advection and vortex stretching. arXiv preprint arXiv:1701.04050, 2017.

[11] CL Fefferman. Existence and smoothness of the Navier-Stokes equation. The millennium prize problems, pages 57-67, 2006.

[12] JD Gibbon. The three-dimensional Euler equations: Where do we stand? Physica D: Nonlinear Phenomena, 237(14):1894-1904, 2008.

[13] GH Golub and CF Van Loan. Matrix Computations. The Johns Hopkins University Press, 1989.

[14] GH Hardy, JE Littlewood, and G Pólya. Inequalities. Cambridge university press, 1952.

[15] TY Hou. Blow-up or no blow-up? a unified computational and analytic approach to 3D incompressible Euler and Navier-Stokes equations. Acta Numerica, 18(1):277-346, 2009.

[16] TY Hou and Z Lei. On the stabilizing effect of convection in three-dimensional incompressible flows. Communications on Pure and Applied Mathematics, 62(4):501-564, 2009.

[17] TY Hou and C Li. Dynamic stability of the three-dimensional axisymmetric Navier-Stokes equations with swirl. Communications on Pure and Applied Mathematics, 61(5):661-697, 2008.

[18] TY Hou and G Luo. On the finite-time blowup of a 1D model for the 3D incompressible Euler equations. arXiv preprint arXiv:1311.2613, 2013.

[19] H Jia, S Stewart, and V Sverak. On the de gregorio modification of the constantinlaxmajda model. ARMA, 231(2):1269-1304, 2019.

[20] A Kiselev. Small scales and singularity formation in fluid dynamics. arXiv:180\%.00184 [math.AP], 2018

[21] G Luo and TY Hou. Toward the finite-time blowup of the 3D incompressible Euler equations: a numerical investigation. SIAM Multiscale Modeling and Simulation, 12(4):1722-1776, 2014.

[22] Ramon E Moore, R Baker Kearfott, and Michael J Cloud. Introduction to interval analysis, volume 110. Siam, 2009.

[23] H Okamoto, T Sakajo, and M Wunsch. On a generalization of the constantinlaxmajda equation. Nonlinearity, 21(10):2447-2461, 2008.

[24] Siegfried M Rump. Verification methods: Rigorous results using floating-point arithmetic. Acta Numerica, 19:287-449, 2010.

[25] S.M. Rump. INTLAB - INTerval LABoratory. In Tibor Csendes, editor, Developments in Reliable Computing, pages 77-104. Kluwer Academic Publishers, Dordrecht, 1999. http://www.ti3.tuhh.de/rump/

Applied and Computational Mathematics, California Institute of Technology, Pasadena, CA 91125, USA 


\title{
SUPPLEMENTARY MATERIALS FOR THE PAPER "ON THE FINITE TIME BLOWUP OF THE DE GREGORIO MODEL FOR THE 3D EULER EQUATIONS"
}

\author{
JIAJIE CHEN, THOMAS Y. HOU, AND DE HUANG
}

\begin{abstract}
In 1, we have presented linear stability analysis for the linearized operator and shown that it has strictly negative spectrum in the weighted $L^{2}+H^{1}$ norm. In the case of $a=1$, we constructed the approximate self-similar profile numerically. Our stability estimate is uniform for all approximate profiles sufficiently close to the exact self-similar solution. In principle, if we have access to a very powerful parallel supercomputer, we can afford to take a sufficiently small mesh size $h$ to construct an approximate self-similar profile with extremely small residual error. This would have completed the bootstrap argument. The purpose of this supplementary material is to provide relatively sharp estimates for the cross term in the weighted $H^{1}$ estimate, the error term and the nonlinear term. These sharp stability and error estimates enable us to complete our bootstrap argument using only a personal laptop computer with a mesh size $h=2 * 10^{-5}$ to construct our approximate self-similar profile. To obtain sharp estimates, we also use the Interval arithmetics software and error analysis for the Trapezoidal rule to estimate a number of integrals involving our approximate self-similar profile with rigorous error control. We will provide the estimates for the cross term in Section 2 the error term in Section 3 and the nonlinear term in Section 4
\end{abstract}

\section{Lemmas, Functions and Notations}

We first introduce some Lemmas, functions and notations that were used in [1].

1.1. Functions. The weight $\varphi$ in the $L^{2}$ estimate is defined as follows:

$$
\varphi \triangleq\left(-\frac{1}{x^{3}}-\frac{e}{x}-\frac{f \cdot 2 x}{L^{2}-x^{2}}\right) \cdot\left(\chi_{1}\left(\bar{\omega}-\frac{x \bar{\omega}_{x}}{5}\right)+\chi_{2}\left(\bar{\omega}-\frac{(x-L) \bar{\omega}_{x}}{3}\right)\right)^{-1}
$$

where $\chi_{1}, \chi_{2} \geq 0$ are some cutoff functions such that $\chi_{1}+\chi_{2}=1$ and

$$
\chi_{1}(x)=\left\{\begin{array}{ll}
1 & x \in[0,4] \\
0 & x \in[6,10]
\end{array}, \quad \chi_{1}(x)=\frac{\exp \left(\frac{1}{x-4}+\frac{1}{x-6}\right)}{1+\exp \left(\frac{1}{x-4}+\frac{1}{x-6}\right)} \forall x \in[4,6] .\right.
$$

The weight $\psi$ in the $H^{1}$ estimate is given below:

$$
\psi=-\frac{1}{\bar{\omega}}\left(\frac{1}{x}-\frac{x}{L^{2}}\right), \quad x \in[0, L] .
$$

The normalization conditions for $c_{l}, c_{\omega}$ are defined as follows:

$$
c_{l}=-\frac{u(L)}{L}, \quad c_{\omega}=c_{l} .
$$

The inner product is defined on the interval $[0, L]$

$$
\langle f, g\rangle \triangleq \int_{0}^{L} f \cdot g d x
$$

since the support of $\omega, \bar{\omega}$ lies in $[-L, L]$. We only consider the half real line due to symmetry.

The nonlinear and the error terms in the weighted $L^{2}$ and $H^{1}$ estimates are defined below:

$$
\begin{aligned}
N(\omega)=\left(c_{\omega}+u_{x}\right) \omega-\left(c_{l} x+u\right) \omega_{x}, & F(\bar{\omega})=\left(\bar{c}_{\omega}+\bar{u}_{x}\right) \bar{\omega}-\left(\bar{c}_{l} x+\bar{u}\right) \bar{\omega}_{x}, \\
N_{1} \triangleq\langle N(\omega), \omega \varphi\rangle, \quad F_{1} \triangleq\langle F(\bar{\omega}), \omega \varphi\rangle, & N_{2} \triangleq\left\langle(N(\omega))_{x}, \omega_{x} \psi\right\rangle, \quad F_{2} \triangleq\left\langle(F(\bar{\omega}))_{x}, \omega \varphi\right\rangle .
\end{aligned}
$$


We express $u_{x}(0), u_{x}(L)$ and $c_{\omega}=c_{l}$ as the projection of $\omega$ onto some functions

$$
\begin{aligned}
& c_{\omega}=c_{l}=\left\langle\omega, g_{c_{\omega}}\right\rangle, \quad u_{x}(0)=\left\langle\omega, g_{u_{x}(0)}\right\rangle, \quad u_{x}(L)=\left\langle\omega, g_{u_{x}(L)}\right\rangle, \\
& g_{c_{\omega}} \triangleq \frac{1}{L \pi} \log \left(\frac{L+x}{L-x}\right), \quad g_{u_{x}(0)} \triangleq-\frac{2}{\pi x}, \quad g_{u_{x}(L)} \triangleq \frac{2 x}{\pi\left(L^{2}-x^{2}\right)} .
\end{aligned}
$$

1.2. Parameters and Notations. We will use the following parameters and notations

$$
n=8000, h_{0}=\frac{L}{n}=\frac{1}{800}, N=400000, h=\frac{L}{N}=2.5 \cdot 10^{-5}, x_{i}=i h, i=0,1, . ., N .
$$

We introduce the following constants which can be estimated accurately

$$
\begin{aligned}
K_{1} \triangleq\left\|\bar{\omega}_{x}||_{L^{\infty}[0, L]}, \quad K_{1 r} \triangleq\right\| \bar{\omega}_{x}\left\|_{L^{\infty}[M, L]}, \quad K_{2} \triangleq\right\| \bar{u}_{x} \|_{L^{\infty}[0, L]}, \\
K_{3} \triangleq \max _{[0, L]}\left|\bar{c}_{l}+\bar{u}_{x}\right|, \quad K_{4} \triangleq\left\|\bar{u}_{x x}\right\|_{L^{\infty}[0, M]}, \\
K_{5 l} \triangleq\left\|\frac{\bar{\omega}}{x\left(x^{2}-L^{2}\right)}\right\|_{L^{\infty}[0, M]}, \quad K_{5} \triangleq\left\|\frac{\bar{\omega}}{\left(x^{2}-L^{2}\right)}\right\|_{L^{\infty}[0, L]}, \\
K_{6} \triangleq\left\|\left(\bar{\omega}_{x x}\left(x^{2}-L^{2}\right)\right)_{x}\right\|_{L^{\infty}[0.25, L-0.25]}, \quad K_{7} \triangleq\left\|\bar{\omega}_{x x}\left(x^{2}-L^{2}\right)\right\|_{L^{\infty}[0, L]}, \quad K_{8}=\left\|\bar{\omega}_{x x}\right\|_{L^{\infty}[0.25, L-0.25]}, \\
J_{1} \triangleq \int_{0}^{M} \bar{\omega}_{x x x}^{2} d x, \quad J_{2} \triangleq \int_{0}^{M} \frac{\bar{\omega}_{x x}^{2}}{x^{2}} d x, \quad J_{3} \triangleq \int_{0}^{L} \frac{\left(\omega_{x}-\omega_{x}(0)\right)^{2}}{x^{2}} d x, \\
J_{4} \triangleq \int_{0}^{L} \bar{\omega}_{x x}^{2}\left(x^{2}-L^{2}\right)^{2} d x, \quad J_{5} \triangleq \int_{0}^{L} \bar{\omega}_{x x x}^{2}(x-L)^{2} d x, \quad J_{5 r} \triangleq \int_{M}^{L} \bar{\omega}_{x x x}^{2}(x-L)^{2} d x, \\
J_{6} \triangleq \int_{0}^{L} \bar{\omega}_{x}^{2} d x, \quad J_{7} \triangleq \int_{0}^{L} \bar{\omega}_{x x}^{2} d x, \quad J_{7 r} \triangleq \int_{M}^{L} \bar{\omega}_{x x}^{2} d x .
\end{aligned}
$$

Using the theoretical estimates and the methods of interval arithmetics introduced in section 3 [1], we can obtain rigorous upper bounds for the positive quantities above. In particular, for each quantity $q$, our numerical verifications using the interval arithmetics will provide an interval $\left[q_{l}, q_{r}\right]$ that rigorously contain the exact value, where $q_{l}, q_{r}$ are two accurate real numbers with 16 digits. We then round up $q_{r}$ to 4 significant digits to be a strict upper bound of $q$. One can verify that

$$
\begin{aligned}
& K_{1}<1.001, \quad K_{1, r}<0.8092, \quad K_{2}<3.595 \quad K_{3}<2.896, \quad K_{4}<0.9295, \\
& K_{5, l} \leq 1.000 \times 10^{-2}, \quad K_{5} \leq 4.433 \times 10^{-2}, \quad K_{6}<11.13, \quad K_{7}<26.65, \quad K_{8}<0.5152, \\
& J_{1}^{1 / 2} \leq 0.1683, \quad J_{2}^{1 / 2} \leq 0.2141, \quad J_{3}^{1 / 2} \leq 0.5757, \quad J_{4}^{1 / 2} \leq 53.37, \quad J_{5}^{1 / 2} \leq 1.960, \\
& J_{5, r}^{1 / 2} \leq 1.960, \quad J_{6}^{1 / 2} \leq 2.096, \quad J_{7}^{1 / 2} \leq 0.6965, \quad J_{7, r}^{1 / 2} \leq 0.4500, \\
& \left\|\left(x^{2}-L^{2}\right)^{-1} \psi^{1 / 2} \varphi^{-1 / 2}\right\|_{L^{\infty}[0.5,9.5]}<0.1, \quad\left\|\psi_{x} \psi^{-1 / 2} \varphi^{-1 / 2}\right\|_{L^{\infty}[0.5,9.5]}<2 .
\end{aligned}
$$

\section{The Cross term in the Weighted $H^{1}$ estimate}

In [1, we have derived the following weighted $H^{1}$ estimate:

$$
\begin{aligned}
\frac{1}{2} \frac{d}{d t}\left\langle\omega_{x}^{2}, \psi\right\rangle & =\left\langle\frac{1}{2 \psi}\left(\left(\bar{c}_{l} x+\bar{u}\right) \psi\right)_{x}, \omega_{x}^{2} \psi\right\rangle+\left\langle\bar{u}_{x x} \omega, \omega_{x} \psi\right\rangle-\left\langle\left(c_{l} x+u\right) \bar{\omega}_{x x}, \omega_{x} \psi\right\rangle \\
& +\left\langle N(\omega)_{x}, \omega_{x} \psi\right\rangle+\left\langle F(\bar{\omega})_{x}, \omega_{x} \psi\right\rangle \triangleq I+I I_{2}+N_{2}+F_{2}
\end{aligned}
$$

where $I, I I_{2}$ are defined below

$$
I=\left\langle\frac{1}{2 \psi}\left(\left(\bar{c}_{l} x+\bar{u}\right) \psi\right)_{x}, \omega_{x}^{2} \psi\right\rangle+\left\langle\bar{u}_{x x} \omega, \omega_{x} \psi\right\rangle, \quad I I_{2}=-\left\langle\left(c_{l} x+u\right) \bar{\omega}_{x x}, \omega_{x} \psi\right\rangle .
$$

We further decompose $I$ into the a damping term and a cross term

$$
I=\left\langle D_{2}(\bar{\omega}), \omega_{x}^{2} \psi\right\rangle+I_{2}, \quad D_{2}(\bar{\omega}) \triangleq \frac{1}{2 \psi}\left(\left(\bar{c}_{l} x+\bar{u}\right) \psi\right)_{x}, \quad I_{2} \triangleq\left\langle\bar{u}_{x x} \omega, \omega_{x} \psi\right\rangle .
$$

In this Section, we estimate the constant for the cross terms $I_{2}, I I_{2}$ in the weighted $H^{1}$ estimate and prove Lemma 2.1. 
Lemma 2.1. The weighted $H^{1}$ estimate satisfies

$$
\frac{1}{2} \frac{d}{d t}\left\langle\omega_{x}^{2}, \psi\right\rangle=I+I I_{2}+N_{2}+F_{2} \leq-0.25\left\langle\omega_{x}^{2}, \psi\right\rangle+7.5\left\langle\omega^{2}, \varphi\right\rangle+N_{2}+F_{2},
$$

where $I, I I_{2}$ are defined in (2.2).

In [1, we have shown that $\left\langle D_{2}(\bar{\omega}), \omega^{2} \psi\right\rangle$ in $I$ is a damping term. To control the cross term, one can use the Cauchy-Schwartz inequality and split it into weighted $L^{2}$ norm of $\omega$ and $\omega_{x}$. However, this estimate is too crude and leads to a large constant in our estimate, which is roughly of order $100\left\langle\omega^{2}, \varphi\right\rangle$, in Lemma 2.1. Then to close the bootstrap argument, it requires the error term to be extremely small, which increases the computational burden. To overcome this difficulty, we will use part of the damping term and some property of the profile to control the cross term, so that we can get a smaller constant in Lemma 2.1, which is about $7.5\left\langle\omega^{2}, \varphi\right\rangle$.

We first consider $I_{2}=\left\langle\bar{u}_{x x} \omega, \omega_{x} \psi\right\rangle$ in $I$ (2.3). Note that $\bar{u}_{x x} \psi$ is increasing on the grid points. Formally, by using integration by parts, we get

$$
I_{2}=\left\langle\bar{u}_{x x} \omega, \omega_{x} \psi\right\rangle=-\frac{1}{2}\left\langle\left(\bar{u}_{x x} \psi\right)_{x}, \omega^{2}\right\rangle \leq 0 .
$$

However, since $\bar{u}_{x x x}$ is not continuous, we cannot justify $\left(\bar{u}_{x x} \psi\right)_{x} \geq 0$. To address this problem, we consider the following piecewise linear approximation of $\bar{u}_{x x} \psi$

$$
S_{k, h}(x) \triangleq \begin{cases}\left(\bar{u}_{x x} \psi\right)(0.5) & 0 \leq x \leq 0.5, \\ \left(\bar{u}_{x x} \psi\right)\left(x_{i}\right)+\left(\left(\bar{u}_{x x} \psi\right)\left(x_{i+1}\right)-\left(\bar{u}_{x x} \psi\right)\left(x_{i}\right)\right) \frac{x-x_{i}}{h} & x \in\left[x_{i}, x_{i+1}\right] \subset[0.5,9.5], \\ \left(\bar{u}_{x x} \psi\right)(9.5) & x \geq 9.5,\end{cases}
$$

where $\bar{u}_{x x}\left(x_{i}\right), x_{i}=i h$ is evaluated using the Hilbert transform (see A.5D)

$$
\bar{u}_{x x}=H \omega_{x}, \quad \bar{u}_{x x}\left(x^{2}-L^{2}\right)=H\left(\bar{\omega}_{x}\left(x^{2}-L^{2}\right)\right) .
$$

For $x$ close to 0 or $L$, we construct $S_{k, h}(x)$ to be constant since $\bar{u}_{x x} \psi$ blows up at $x=0, L$. We can verify rigorously that $S_{k, h}(x)$ is monotonically increasing by checking its values on the grid points since $S_{k, h}$ is piecewisely linear. Using the fact that $S_{k, h}(x)$ is monotonically increasing, we can justify (2.5) as follows

$$
\begin{aligned}
\left\langle\bar{u}_{x x} \omega, \omega_{x} \psi\right\rangle & =\left\langle\bar{u}_{x x} \psi-S_{k, h}(x), \omega \omega_{x}\right\rangle+\left\langle S_{k, h}(x), \omega \omega_{x}\right\rangle \\
& =\left\langle\left(\bar{u}_{x x} \psi-S_{k, h}(x)\right) \varphi^{-1 / 2} \psi^{-1 / 2}, \varphi^{1 / 2} \omega \psi^{1 / 2} \omega_{x}\right\rangle-\frac{1}{2}\left\langle\partial_{x} S_{k, h}(x), \omega^{2}\right\rangle \\
& \leq\left\|\left(\bar{u}_{x x} \psi-S_{k, h}(x)\right) \varphi^{-1 / 2} \psi^{-1 / 2}\right\|_{L^{\infty}}\left\|\omega \varphi^{1 / 2}\right\|_{L^{2}}\left\|\omega_{x} \psi^{1 / 2}\right\|_{L^{2}}-\frac{1}{2}\left\langle\partial_{x} S_{k, h}(x), \omega^{2}\right\rangle .
\end{aligned}
$$

Next we estimate the error of linear interpolation. We have plotted the numerical values of $S_{k, h}$ and $\Delta(x)$ defined below on the grid points in the first subfigure in Figure 1 in the Appendix.

Lemma 2.2. The weighted approximation error satisfies

$$
\left\|\left(\bar{u}_{x x} \psi-S_{k, h}(x)\right) \varphi^{-1 / 2} \psi^{-1 / 2}\right\|_{L^{\infty}} \leq 0.1 .
$$

Proof. Define

$$
\Delta(x) \triangleq\left(\bar{u}_{x x} \psi-S_{k, h}(x)\right) \varphi^{-1 / 2} \psi^{-1 / 2} .
$$

For $x \leq 0.5$ or $x \geq 9.5$, we use the interval arithmetics computation to show that the maximal value of $\Delta(x)$ on the grid points is bounded by 0.09. In (2.10), (2.11), we show that $\bar{u}_{x x}, \bar{u}_{x x x}\left(x^{2}-\right.$ $L^{2}$ ) grow at most logarithmically near $x=L$. Using the asymptotic property of the weight

$$
\psi \asymp x^{-2}+1, \varphi \asymp x^{-4}+(x-L)^{-2},
$$

we obtain that

$$
\psi^{1 / 2} \varphi^{-1 / 2}, \psi^{-1 / 2} \varphi^{-1 / 2} \lesssim\left(x^{-1}+(x-L)^{-1}\right)^{-1},
$$

which vanishes at $x=0, L$. For $x$ sufficiently close to $x=L$, e.g. $|x-L|<\varepsilon$, we can use the fact that $\Delta(x)$ vanishes at $x=L$ and that $\Delta(x)$ is at least $C^{1 / 2}$ to verify $|\Delta(x)| \leq 0.1$. For $|x-L|>\varepsilon$, we can use the smoothness of $\psi, \varphi \in C^{1,1}$ and $u_{x x} \in C^{1}$ to verify $|\Delta(x)| \leq 0.1$ for $x$ that is not on a grid point. 
For $x \in\left[x_{i}, x_{i+1}\right] \triangleq I_{i} \subset[0.5,9.5]$, we use the standard approximation theory to show that

$$
\begin{aligned}
\left|\bar{u}_{x x} \psi-S_{k, h}(x)\right| & \leq h \max _{I_{i}}\left|\left(\bar{u}_{x x} \psi\right)_{x}\right|=h \max _{I_{i}}\left(\left|\bar{u}_{x x x}\left(x^{2}-L^{2}\right) \psi\left(x^{2}-L^{2}\right)^{-1}\right|+\left|\bar{u}_{x x} \psi_{x}\right|\right) \\
& \leq h \max _{I_{i}}\left|\bar{u}_{x x x}\left(x^{2}-L^{2}\right)\right| \max _{I_{i}}\left|\psi\left(x^{2}-L^{2}\right)^{-1}\right|+h \max _{I_{i}}\left|\bar{u}_{x x}\right| \max _{I_{i}}\left|\psi_{x}\right| .
\end{aligned}
$$

Let $\delta=0.25$. From the relation (2.6), we have for $x \in[0.5,9.5]$ that

$$
\begin{aligned}
& \bar{u}_{x x x}(x)\left(x^{2}-L^{2}\right)=P . V \cdot \frac{1}{\pi} \int_{-L}^{L} \frac{\bar{\omega}_{x x}(y)\left(y^{2}-L^{2}\right)}{x-y} d y \\
= & \frac{1}{\pi} \int_{|x-y|<\delta} \frac{\bar{\omega}_{x x}(y)\left(y^{2}-L^{2}\right)-\bar{\omega}_{x x}(x)\left(x^{2}-L^{2}\right)}{x-y} d y+\frac{1}{\pi} \int_{|x-y|>\delta,|y| \leq L} \frac{\bar{\omega}_{x x}(y)\left(y^{2}-L^{2}\right)}{x-y} d y,
\end{aligned}
$$

which implies

$$
\begin{aligned}
\left|\bar{u}(x)_{x x x}\left(x^{2}-L^{2}\right)\right| & \leq \frac{2 \delta}{\pi}\left\|\left(\bar{\omega}_{x x}\left(x^{2}-L^{2}\right)\right)_{x}\right\|_{L^{\infty}[0.5-\delta, 9.5+\delta]}+\frac{2}{\pi}\left\|\bar{\omega}_{x x}\left(y^{2}-L^{2}\right)\right\|_{\infty} \log \frac{2 L}{\delta} \\
& =\frac{2 \delta}{\pi} K_{6}+\frac{2}{\pi} K_{7} \log \frac{2 L}{\delta},
\end{aligned}
$$

where $K_{6}, K_{7}$ are defined in (1.6). Similarly, for $\bar{u}_{x x}=H \bar{\omega}_{x}$, we have

$$
\left|\bar{u}_{x x}(x)\right| \leq \frac{2 \delta}{\pi}\left\|\bar{\omega}_{x x}\right\|_{L^{\infty}[0.5-\delta, 9.5+\delta]}+\frac{2}{\pi}\left\|\bar{\omega}_{x}\right\|_{\infty} \log \frac{2 L}{\delta}=\frac{2 \delta}{\pi} K_{8}+\frac{2}{\pi} K_{1} \log \frac{2 L}{\delta},
$$

where $K_{8}, K_{1}$ are defined in (1.6). Hence, for $x \in[0.5,9.5], \bar{u}_{x x}, \bar{u}_{x x x}$ are bounded. Taking $\delta=$ $L-x$ in (2.10) and (2.11), we see that both $\bar{u}_{x x x}\left(x^{2}-L^{2}\right), \bar{u}_{x x}$ can grow at most logarithmically near $x=L$ with another constant in the upper bound. For $x \in\left[x_{i}, x_{i+1}\right] \subset[0.5,9.5]$, one can verify that $\psi$ and $\varphi$ remain smooth over one grid cell. Specifically, we have the following estimate:

$$
\max _{I_{i}}\left|\psi_{x}\right| \leq 1.1\left|\psi_{x}\left(x_{i}\right)\right|, \max _{I_{i}}\left|\psi\left(x^{2}-L^{2}\right)^{-1}\right| \leq 1.1\left|\psi\left(x_{i}\right)\left(x_{i}^{2}-L^{2}\right)^{-1}\right| .
$$

Substituting the bounds on $\bar{u}_{x x}, \bar{u}_{x x x}$ in (2.10), (2.11) with $\delta=0.25$ and (2.12) into (2.9), we obtain

$$
\begin{aligned}
\max _{[0.5,9.5]}|\Delta(x)| \leq & 1.1 h \frac{2}{\pi}\left(\delta K_{6}+K_{7} \log \frac{2 L}{\delta}\right)\left\|\left(x^{2}-L^{2}\right)^{-1} \psi^{1 / 2} \varphi^{-1 / 2}\right\|_{L^{\infty}[0.5,9.5]} \\
& +1.1 h \frac{2}{\pi}\left(\delta K_{8}+K_{1} \log \frac{2 L}{\delta}\right)\left\|\psi_{x} \psi^{-1 / 2} \varphi^{-1 / 2}\right\|_{L^{\infty}[0.5,9.5]} \\
\leq & 84 h\left\|\left(x^{2}-L^{2}\right)^{-1} \psi^{1 / 2} \varphi^{-1 / 2}\right\|_{L^{\infty}[0.5,9.5]}+3.2 h\left\|\psi_{x} \psi^{-1 / 2} \varphi^{-1 / 2}\right\|_{L^{\infty}[0.5,9.5]} \\
\leq & (84 \cdot 0.1+3.2 \cdot 2) h<0.1
\end{aligned}
$$

where we have used (1.7) and $h=2.5 \times 10^{-5}$. Combining all the above estimate would give the desired upper bound $|\Delta(x)| \leq 0.1, \forall x \in[0, L]$.

Using Lemma 2.2, we can estimate (2.7) below

$$
I_{2}=\left\langle\bar{u}_{x x} \omega, \omega_{x} \psi\right\rangle \leq 0.1\left\|\omega \varphi^{1 / 2}\right\|_{L^{2}}\left\|\omega_{x} \psi^{1 / 2}\right\|_{L^{2}} \leq d_{0}\left\|\omega \varphi^{1 / 2}\right\|_{L^{2}}^{2}+\frac{0.01}{4 d_{0}}\left\|\omega_{x} \psi^{1 / 2}\right\|_{L^{2}}^{2} .
$$

Next, we consider the remaining linear term $I I_{2}=\left\langle\left(c_{l} x+u\right) \bar{\omega}_{x x}, \omega_{x} \psi\right\rangle$ in (2.1). First of all, using integration by parts, we yield

$$
I I_{2}=-\left\langle\left(c_{l} x+u\right) \bar{\omega}_{x x}, \omega_{x} \psi\right\rangle=\left\langle\left(c_{l}+u_{x}\right) \bar{\omega}_{x x} \psi, \omega_{x}\right\rangle+\left\langle\left(c_{l} x+u\right)\left(\bar{\omega}_{x x} \psi\right)_{x}, \omega\right\rangle \triangleq T_{1}+T_{2} .
$$

We use a strategy similar to the one that we performed the weighted $L^{2}$ estimate for $a=1$ in [1]. For $T_{1}$, we first find a combination of power $x, x^{-1}$ that approximates $\bar{\omega}_{x x} \psi$

$$
\begin{aligned}
T_{1} & =\left\langle u_{x} \bar{\omega}_{x x} \psi, \omega\right\rangle+c_{l}\left\langle\bar{\omega}_{x x} \psi, \omega\right\rangle=\left\langle u_{x}\left(\frac{d_{1}}{x}-d_{2} x\right), \omega\right\rangle+\left\langle u_{x}\left(\bar{\omega}_{x x} \psi-\frac{d_{1}}{x}+d_{2} x\right), \omega\right\rangle \\
& +c_{l}\left\langle\bar{\omega}_{x x} \psi-\frac{d_{3}}{x}, \omega\right\rangle+c_{l} d_{3}\left\langle\frac{1}{x}, \omega\right\rangle
\end{aligned}
$$


where $d_{1}, d_{2}, d_{3}>0$ are to be determined. By using the cancellation property (A.6), we get

$$
\begin{aligned}
d_{1}\left\langle u_{x} \omega, x^{-1}\right\rangle & =d_{1}\left(u_{x}(0)\left\langle\omega, x^{-1}\right\rangle+\left\langle\left(u_{x}-u_{x}(0)\right) \omega, x^{-1}\right\rangle\right) \\
& =d_{1}\left(-u_{x}(0) \frac{\pi}{2}(H \omega)(0)+\frac{\pi}{4} u_{x}(0)^{2}\right)=-\frac{\pi d_{1}}{4} u_{x}(0)^{2},
\end{aligned}
$$

where we have a factor $1 / 2$ since the integral is from 0 to $L$ rather than the real line. Using $x u_{x}=H(x \omega)$ in (A.4) and (A.1), we derive

$$
\begin{aligned}
\left\langle u_{x} \omega, x\right\rangle & =\left\langle H(x \omega)(x \omega), x^{-1}\right\rangle=-\frac{\pi}{2} H(H(x \omega)(x \omega)) \\
& =-\frac{\pi}{4}\left((H(x \omega))^{2}(0)-(x \omega)^{2}(0)\right)=-\frac{\pi}{4}(H(x \omega))^{2}(0)=-\frac{\pi}{4}\left(x u_{x}\right)^{2}(0)=0 .
\end{aligned}
$$

Using an elementary inequality $2 a b \leq a^{2}+b^{2}$, we get

$$
c_{l} d_{3}\left\langle x^{-1}, \omega\right\rangle=-\frac{\pi}{2} c_{l} d_{3} u_{x}(0) \leq \frac{\pi d_{1}}{4} u_{x}^{2}(0)+\frac{\pi d_{3}^{2}}{4 d_{1}} c_{l}^{2} .
$$

Using the above estimates, we can reduce $T_{1}$ to

$$
\begin{aligned}
T_{1} & \leq-\frac{\pi d_{1}}{4} u_{x}^{2}(0)+\left\langle u_{x}\left(\bar{\omega}_{x x} \psi-\frac{d_{1}}{x}+d_{2} x\right), \omega\right\rangle+c_{l}\left\langle\bar{\omega}_{x x} \psi-\frac{d_{3}}{x}, \omega\right\rangle+\frac{\pi d_{1}}{4} u_{x}^{2}(0)+\frac{\pi d_{3}^{2}}{4 d_{1}} c_{l}^{2} \\
& \leq\left\langle u_{x}\left(\bar{\omega}_{x x} \psi-\frac{d_{1}}{x}+d_{2} x\right), \omega\right\rangle+c_{l}\left\langle\bar{\omega}_{x x} \psi-\frac{d_{3}}{x}, \omega\right\rangle+\frac{\pi d_{3}^{2}}{4 d_{1}} c_{l}^{2} \\
& =\left\langle u_{x}\left(\bar{\omega}_{x x} \psi-\frac{d_{1}}{x}+d_{2} x\right), \omega\right\rangle+c_{l}\left\langle\bar{\omega}_{x x} \psi-\frac{d_{3}}{x}+\frac{\pi d_{3}^{2}}{4 d_{1}} g_{c_{\omega}}, \omega\right\rangle \\
& \leq\left\|u_{x}\right\|_{2}\left\|\left(\bar{\omega}_{x x} \psi-\frac{d_{1}}{x}+d_{2} x\right) \omega\right\|_{2}+c_{l}\left\langle\bar{\omega}_{x x} \psi-\frac{d_{3}}{x}+\frac{\pi d_{3}^{2}}{4 d_{1}} g_{c_{\omega}}, \omega\right\rangle \\
& =\|\omega\|_{2}\left\|\left(\bar{\omega}_{x x} \psi-\frac{d_{1}}{x}+d_{2} x\right) \omega\right\|_{2}+c_{l}\left\langle\bar{\omega}_{x x} \psi-\frac{d_{3}}{x}+\frac{\pi d_{3}^{2}}{4 d_{1}} g_{c_{\omega}}, \omega\right\rangle,
\end{aligned}
$$

where $g_{c_{\omega}}$ defined in (1.5) satisfies $c_{l}=c_{\omega}=\left\langle\omega, g_{c_{\omega}}\right\rangle$ and we have used $\left\|u_{x}\right\|_{2}=\|\omega\|_{2}$. The second term will be estimated later.

To estimate $T_{2}$ in (2.14), we again use a strategy similar to the one that we performed the weighted $L^{2}$ estimate for $a=1$ in [1] by subtracting the different linear part near $x=0$ and $x=L$. Let $M_{2} \in(0, L)$ be some parameter to be chosen later. For $x \in\left[0, M_{2}\right]$, we have

$$
\begin{aligned}
T_{2 l} & \triangleq\left\langle\left(c_{l} x+u\right)\left(\bar{\omega}_{x x} \psi\right)_{x} \mathbf{1}_{x \leq M_{2}}, \omega\right\rangle=\left\langle\left(u-u_{x}(0) x\right)\left(\bar{\omega}_{x x} \psi\right)_{x} \mathbf{1}_{x \leq M_{2}}, \omega\right\rangle \\
& +\left(c_{l}+u_{x}(0)\right)\left\langle x\left(\bar{\omega}_{x x} \psi\right)_{x} \mathbf{1}_{x \leq M_{2}}, \omega\right\rangle \triangleq T_{2 l, 1}+T_{2 l, 2} .
\end{aligned}
$$

We first use the Cauchy-Schwartz inequality to split the first term and then apply the Hardy inequality (A.7)

$$
\begin{aligned}
T_{2 l, 1} & =\left\langle\left(u-u_{x}(0)\right) \sqrt{25 d_{4} x^{-6}+9 d_{5} x^{-4}}, \sqrt{25 d_{4} x^{-6}+9 d_{5} x^{-4}}{ }^{-1}\left(\bar{\omega}_{x x} \psi\right)_{x} \mathbf{1}_{x \leq M_{2}} \omega\right\rangle \\
& \leq \frac{1}{4}\left\langle\left(u-u_{x}(0)\right)^{2}, 25 d_{4} x^{-6}+9 d_{5} x^{-4}\right\rangle+\left\langle\left(25 d_{4} x^{-6}+9 d_{5} x^{-4}\right)^{-1}\left(\left(\bar{\omega}_{x x} \psi\right)_{x}\right)^{2} \mathbf{1}_{x \leq M_{2}}, \omega^{2}\right\rangle \\
& \leq d_{4}\left\langle\omega^{2}, x^{-4}\right\rangle+d_{5}\left\langle\omega^{2}, x^{-2}\right\rangle+\left\langle\left(25 d_{4} x^{-6}+9 d_{5} x^{-4}\right)^{-1}\left(\left(\bar{\omega}_{x x} \psi\right)_{x}\right)^{2} \mathbf{1}_{x \leq M_{2}}, \omega^{2}\right\rangle,
\end{aligned}
$$

where $d_{4}, d_{5}>0$ are to be determined. The constants $25 / 4,9 / 4$ come from (A.7) with $p=4,2$. $T_{2 l, 2}$ will be estimated later.

For $x \in\left[M_{2}, L\right]$, we use (1.3) to rewrite $c_{l} x+u$ and $T_{2 r}$

$$
\begin{aligned}
c_{l} x+u & =u-u(L)+c_{l}(x-L)=u-u(L)-u_{x}(L)(x-L)+\left(c_{l}+u_{x}(L)\right)(x-L) . \\
T_{2 r} & \triangleq\left\langle\left(c_{l} x+u\right)\left(\bar{\omega}_{x x} \psi\right)_{x} \mathbf{1}_{x>M_{2}}, \omega\right\rangle=\left\langle u-u(L)-u_{x}(L)(x-L),\left(\bar{\omega}_{x x} \psi\right)_{x} \mathbf{1}_{x>M_{2}} \omega\right\rangle \\
& +\left(c_{l}+u_{x}(L)\right)\left\langle(x-L)\left(\bar{\omega}_{x x} \psi\right)_{x} \mathbf{1}_{x>M_{2}}, \omega\right\rangle \triangleq T_{2 r, 1}+T_{2 r, 2} .
\end{aligned}
$$


Notice that $u-u(L)-u_{x}(L)(x-L)$ and its derivative vanishes at $x=L$. We apply the Cauchy-Schwartz inequality and then the Hardy inequality similar to (A.7) with $p=2$ to yield

$$
\begin{aligned}
& T_{2 r, 1}=\left\langle\left(u-u(L)-u_{x}(L)(x-L)\right)(x-L)^{-2},(x-L)^{2}\left(\bar{\omega}_{x x} \psi\right)_{x} \mathbf{1}_{x>M_{2}}, \omega\right\rangle \\
\leq & \frac{9 d_{6}}{4}\left\langle\left(u-u(L)-u_{x}(L)(x-L)\right)^{2},(x-L)^{-4}\right\rangle+\frac{1}{9 d_{6}}\left\langle\left((x-L)^{2}\left(\bar{\omega}_{x x} \psi\right)_{x} \mathbf{1}_{x>M_{2}}\right)^{2}, \omega^{2}\right\rangle \\
\leq & d_{6}\left\langle\left(u_{x}-u_{x}(L)\right)^{2},(x-L)^{-2}\right\rangle+\frac{1}{9 d_{6}}\left\langle\left((x-L)^{2}\left(\bar{\omega}_{x x} \psi\right)_{x} \mathbf{1}_{x>M_{2}}\right)^{2}, \omega^{2}\right\rangle .
\end{aligned}
$$

Furthermore, we apply (A.3) and the $L^{2}$ isometry of the Hilbert transform to estimate $\left\langle\left(u_{x}-\right.\right.$ $\left.\left.u_{x}(L)\right)^{2},(x-L)^{-2}\right\rangle$

$$
\left\langle\left(u_{x}-u_{x}(L)\right)^{2},(x-L)^{-2}\right\rangle \leq \int_{\mathbb{R}}\left(u_{x}-u_{x}(L)\right)^{2}(x-L)^{-2} d x=\left\langle\omega^{2},(x-L)^{-2}+(x+L)^{-2}\right\rangle,
$$

which implies

$$
T_{2 r, 1} \leq d_{6}\left\langle\omega^{2},(x-L)^{-2}+(x+L)^{-2}\right\rangle+\frac{1}{9 d_{6}}\left\langle\left((x-L)^{2}\left(\bar{\omega}_{x x} \psi\right)_{x} \mathbf{1}_{x>M_{2}}\right)^{2}, \omega^{2}\right\rangle .
$$

We use the damping of $\omega_{x}$ to control part of the above terms. Applying the Hardy inequality similar to (A.7) with $u-u_{x}(0) x$ replaced by $\omega$ and $p=2$, we obtain

$$
\left\langle\omega^{2}, x^{-4}\right\rangle \leq \frac{4}{9}\left\langle\omega_{x}^{2}, x^{-2}\right\rangle, \quad 0 \leq \frac{4 d_{7}}{9}\left\langle\omega_{x}^{2}, x^{-2}\right\rangle-d_{7}\left\langle\omega^{2}, x^{-4}\right\rangle,
$$

where $d_{7}>0$ is a parameter to be determined. Therefore, using the relation $I I_{2}=T_{1}+T_{2}=$ $T_{1}+T_{2 l, 1}+T_{2 l, 2}+T_{2 r, 1}+T_{2 r, 2}$ and combining all the estimates of $I_{2}, I I_{2}$ in the weighted $H^{1}$ estimate, (2.1), (2.3), (2.13), (2.14), (2.15), (2.16), (2.17), (2.18), (2.19) and (2.20), we derive

$$
\begin{aligned}
& I+I I_{2}=\left\langle D_{2}(\bar{\omega}), \omega_{x}^{2} \psi\right\rangle+I_{2}+T_{1}+T_{2 l, 1}+T_{2 l, 2}+T_{2 r, 1}+T_{2 r, 2} \\
& \leq\left\langle D_{2}(\bar{\omega}), \omega_{x}^{2} \psi\right\rangle+d_{0}\left\|\omega \varphi^{1 / 2}\right\|_{L^{2}}^{2}+\frac{0.01}{4 d_{0}}\left\|\omega_{x} \psi^{1 / 2}\right\|_{L^{2}}^{2} \\
& +\|\omega\|_{2}\left\|\left(\bar{\omega}_{x x} \psi-\frac{d_{1}}{x}+d_{2} x\right) \omega\right\|_{2}+c_{l}\left\langle\bar{\omega}_{x x} \psi-\frac{d_{3}}{x}+\frac{\pi d_{3}^{2}}{4 d_{1}} g_{c_{\omega}}, \omega\right\rangle \\
& +d_{4}\left\langle\omega^{2}, x^{-4}\right\rangle+d_{5}\left\langle\omega^{2}, x^{-2}\right\rangle+\left\langle\left(25 d_{4} x^{-6}+9 d_{5} x^{-4}\right)^{-1}\left(\left(\bar{\omega}_{x x} \psi\right)_{x}\right)^{2} \mathbf{1}_{x \leq M_{2}}, \omega^{2}\right\rangle \\
& +\left(c_{l}+u_{x}(0)\right)\left\langle x\left(\bar{\omega}_{x x} \psi\right)_{x} \mathbf{1}_{x \leq M_{2}}, \omega\right\rangle+d_{6}\left\langle\omega^{2},(x-L)^{-2}+(x+L)^{-2}\right\rangle \\
& +\frac{1}{9 d_{6}}\left\langle\left((x-L)^{2}\left(\bar{\omega}_{x x} \psi\right)_{x} \mathbf{1}_{x>M_{2}}\right)^{2}, \omega^{2}\right\rangle+\left(c_{l}+u_{x}(L)\right)\left\langle(x-L)\left(\bar{\omega}_{x x} \psi\right)_{x} \mathbf{1}_{x>M_{2}}, \omega\right\rangle \\
& +\left(\frac{4 d_{7}}{9}\left\langle\omega_{x}^{2}, x^{-2}\right\rangle-d_{7}\left\langle\omega^{2}, x^{-4}\right\rangle\right) .
\end{aligned}
$$

The quantities on the right hand side can be classified into three classes: $(a)\left\langle\omega_{x}^{2}, f\right\rangle ;(b)\left\langle\omega^{2}, g\right\rangle$ (except $\left.\|\omega\|_{2}\left\|\left(\bar{\omega}_{x x} \psi-\frac{d_{1}}{x}+d_{2} x\right) \omega\right\|_{2}\right)$; (c) Projections of $\omega$, i.e. $\langle\omega, g\rangle$. Using the estimate below

$$
\|\omega\|_{2}\left\|\left(\bar{\omega}_{x x} \psi-\frac{d_{1}}{x}+d_{2} x\right) \omega\right\|_{2} \leq d_{8}\|\omega\|_{2}^{2}+\frac{1}{4 d_{8}}\left\|\left(\bar{\omega}_{x x} \psi-\frac{d_{1}}{x}+d_{2} x\right) \omega\right\|_{2}^{2}
$$


we can rewrite 2.21) as

$$
\begin{aligned}
I+I I_{2} & \leq\left\langle S_{1}, \omega_{x}^{2} \psi\right\rangle+\left\langle S_{2}, \omega^{2} \varphi\right\rangle+P(\omega) \\
S_{1} & =D_{2}(\bar{\omega})+\frac{0.01}{4 d_{0}}+\left(\frac{4 d_{7}}{9} x^{-2}\right) \psi^{-1}, \\
S_{2} \varphi & =d_{0} \varphi+d_{8}+\frac{1}{4 d_{8}}\left(\bar{\omega}_{x x} \psi-\frac{d_{1}}{x}+d_{2} x\right)^{2}+d_{4} x^{-4}+d_{5} x^{-2} \\
& +\left(25 d_{4} x^{-6}+9 d_{5} x^{-4}\right)^{-1}\left(\left(\bar{\omega}_{x x} \psi\right)_{x}\right)^{2} \mathbf{1}_{x \leq M_{2}}+d_{6}\left((x-L)^{-2}+(x+L)^{-2}\right) \\
& +\frac{1}{9 d_{6}}\left((x-L)^{2}\left(\bar{\omega}_{x x} \psi\right)_{x} \mathbf{1}_{x>M_{2}}\right)^{2}-d_{7} x^{-4}, \\
P(\omega) & \triangleq c_{l}\left\langle R_{1}, \omega\right\rangle+\left(c_{l}+u_{x}(0)\right)\left\langle R_{2}, \omega\right\rangle+\left(c_{l}+u_{x}(L)\right)\left\langle R_{3}, \omega\right\rangle, \\
R_{1} & =\bar{\omega}_{x x} \psi-\frac{d_{3}}{x}+\frac{\pi d_{3}^{2}}{4 d_{1}} g_{c_{\omega}}, R_{2}=x\left(\bar{\omega}_{x x} \psi\right)_{x} \mathbf{1}_{x \leq M_{2}}, R_{3}=(x-L)\left(\bar{\omega}_{x x} \psi\right)_{x} \mathbf{1}_{x>M_{2}} .
\end{aligned}
$$

Finally, we estimate $P(\omega)$. Using (1.5), we can rewrite $P(\omega)$ as follows

$$
P(\omega)=\left\langle g_{c_{\omega}}, \omega\right\rangle\left\langle R_{1}, \omega\right\rangle+\left\langle g_{c_{\omega}}+g_{u_{x}(0)}, \omega\right\rangle\left\langle R_{2}, \omega\right\rangle+\left\langle g_{c_{\omega}}+g_{u_{x}(L)}, \omega\right\rangle\left\langle R_{3}, \omega\right\rangle .
$$

For some function $S_{3} \in C([0, L]), S_{3}>0$ to be determined, we want to bound $P(\omega)$ by $\left\langle S_{3}, \omega^{2} \varphi\right\rangle$ as sharp as possible. We define

$$
\begin{aligned}
& z \triangleq\left(S_{3} \varphi\right)^{1 / 2} \omega, \eta_{1} \triangleq\left(S_{3} \varphi\right)^{-1 / 2} g_{c_{\omega}}, \eta_{2} \triangleq\left(S_{3} \varphi\right)^{-1 / 2} R_{1}, \eta_{3} \triangleq\left(S_{3} \varphi\right)^{-1 / 2}\left(g_{c_{\omega}}+g_{u_{x}(0)}\right), \\
& \eta_{4} \triangleq\left(S_{3} \varphi\right)^{-1 / 2} R_{2}, \quad \eta_{5} \triangleq\left(S_{3} \varphi\right)^{-1 / 2}\left(g_{c_{\omega}}+g_{u_{x}(L)}\right), \quad \eta_{6} \triangleq\left(S_{3} \varphi\right)^{-1 / 2} R_{3} .
\end{aligned}
$$

We want to find the best constant of the following inequality for any $\omega \in L^{2}(\varphi)$

$$
\left\langle\eta_{1}, z\right\rangle\left\langle\eta_{2}, z\right\rangle+\left\langle\eta_{3}, z\right\rangle\left\langle\eta_{4}, z\right\rangle+\left\langle\eta_{5}, z\right\rangle\left\langle\eta_{6}, z\right\rangle \leq C^{*}\|z\|_{2}^{2},
$$

which is equivalent to

$$
P(\omega)=\left\langle g_{c_{\omega}}, \omega\right\rangle\left\langle R_{1}, \omega\right\rangle+\left\langle g_{c_{\omega}}+g_{u_{x}(0)}, \omega\right\rangle\left\langle R_{2}, \omega\right\rangle+\left\langle g_{c_{\omega}}+g_{u_{x}(L)}, \omega\right\rangle\left\langle R_{3}, \omega\right\rangle \leq C^{*}\left\langle S_{3}, \omega^{2} \varphi\right\rangle .
$$

It is easy to see that $\eta_{i}$ are generically linearly independent. Suppose that $V \triangleq \operatorname{span}\left\{\eta_{1}, \eta_{2}, . ., \eta_{6}\right\}$ and $\left\{e_{i}\right\}_{1}^{6}$ is an orthonormal basis of $V$ under the standard $L^{2}$ inner product on $[0, L]$. Suppose that $\eta_{i}$ in terms of the orthonormal basis $\left\{e_{i}\right\}_{1}^{6}$ has the coordinate $\theta_{i} \in \mathbb{R}^{6}$ (a column vector). We can apply the same analysis as we analyzed the best constant of the projection term in the $L^{2}$ estimate for $a=1$ in [1] to obtain that

$$
C^{*}=\lambda_{\max }\left(\frac{1}{2} \sum_{i=1}^{3}\left(\theta_{2 i-1} \theta_{2 i}^{T}+\theta_{2 i} \theta_{2 i-1}^{T}\right)\right), \quad \frac{1}{2} \sum_{i=1}^{3}\left(\theta_{2 i-1} \theta_{2 i}^{T}+\theta_{2 i} \theta_{2 i-1}^{T}\right) \in R^{6 \times 6} .
$$

2.1. Optimizing the parameters. To optimize the estimate, we choose

$$
\begin{aligned}
& d_{0}=0.15, d_{1}=0.11, d_{2}=0.0013, d_{3}=0.07, d_{4}=4.5, d_{5}=0.05, \\
& d_{6}=0.03, d_{7}=2.5, d_{8}=0.0004, M_{2}=6.5 .
\end{aligned}
$$

After specifying these parameters, the remaining part of the damping term $S_{1}$ (see (2.22) ) and the estimate of the cross term $S_{2}$ are completely determined. We plot the numerical values of $S_{1}, S_{2}$ on the grid points in the second subfigure of Figure 1 in the Appendix.

The numerical value of $\max \left(S_{1}\right)$ is less than -0.3 and we use a conservative estimate

$$
S_{1}<-0.25
$$

which can be verified using the smoothness of the profile. It is clear that $S_{2}<7$ and we choose

$$
S_{3}=7.5-S_{2} \text {. }
$$

The corresponding $\eta_{i}$ in (2.23) are determined. The optimal constant in (2.24) can be computed via (2.26). The numerical value of $C^{*}$ satisfies $C^{*}<0.85$ and we use a conservative estimate $C^{*}<1$, which can be verified rigorously. Plugging this estimate into (2.25), we obtain

$$
P(\omega) \leq C^{*}\left\langle S_{3}, \omega^{2} \varphi\right\rangle<\left\langle S_{3}, \omega^{2}\right\rangle=\left\langle 7.5-S_{2}, \omega^{2} \varphi\right\rangle .
$$


Plugging the above estimate and (2.27) into (2.22), we prove

$$
I+I I_{2} \leq\left\langle S_{1}, \omega_{x}^{2} \psi\right\rangle+\left\langle S_{2}, \omega^{2} \varphi\right\rangle+P(\omega) \leq-0.25\left\langle\omega_{x}^{2}, \psi\right\rangle+7.5\left\langle\omega^{2}, \varphi\right\rangle,
$$

which completes the proof of Lemma 2.1 ,

\section{The estimates OF THE ERROR TERM}

The error terms in the weighted $L^{2}, H^{1}$ estimate are given below

$$
F_{1}=\langle F(\bar{\omega}), \omega \varphi\rangle, \quad F_{2}=\left\langle(F(\bar{\omega}))_{x}, \omega_{x} \psi\right\rangle, \quad F(\bar{\omega})=\left(\bar{c}_{\omega}+\bar{u}_{x}\right) \bar{\omega}-\left(\bar{c}_{l} x+\bar{u}\right) \bar{\omega}_{x},
$$

where $\varphi, \psi$ are defined in (1.1) and (1.2), respectively. For some functions $\rho_{1}, \rho_{2}$ to be determined, the Cauchy-Schwartz inequality implies

$$
\left|F_{1}\right| \leq\left\langle F(\bar{\omega})^{2}, \rho_{1}\right\rangle^{1 / 2}\left\langle\omega^{2}, \varphi^{2} \rho_{1}^{-1}\right\rangle^{1 / 2}, \quad\left|F_{2}\right| \leq\left\langle(F(\bar{\omega}))_{x}^{2}, \rho_{2}\right\rangle^{1 / 2}\left\langle\omega_{x}^{2}, \psi^{2} \rho_{2}^{-1}\right\rangle^{1 / 2} .
$$

Our goal is to verify that $\left\langle F(\bar{\omega})^{2}, \rho_{1}\right\rangle^{1 / 2},\left\langle(F(\bar{\omega}))_{x}^{2}, \rho_{2}\right\rangle^{1 / 2}$ are small. Note that we can only evaluate $u, u_{x}, u_{x x}$ at finitely many points via the Hilbert transform. We will use the composite Trapezoidal rule to approximate the integral and have the following error estimate for the Trapezoidal rule.

\subsection{Error estimate of the Trapezoidal rule.}

Lemma 3.1 (Error estimate for the Trapezoidal rule).

$$
\begin{aligned}
\int_{0}^{M} \frac{F^{2}}{x^{4}} d x-T_{h}\left(\frac{F^{2}}{x^{4}}, 0, M\right) & \leq \frac{h^{2}}{4}\left(\int_{0}^{M} \frac{F_{x x}^{2}}{x^{2}} d x\right)^{1 / 2}\left(\int_{0}^{M} \frac{F^{2}}{x^{6}} d x\right)^{1 / 2} \\
\int_{M}^{L} \frac{F^{2}}{(x-L)^{2}} d x-T_{h}\left(\frac{F^{2}}{(x-L)^{2}}, M, L\right) & \leq \frac{h^{2}}{4}\left(\int_{M}^{L} F_{x x}^{2} d x\right)^{1 / 2}\left(\int_{M}^{L} \frac{F^{2}}{(x-L)^{4}} d x\right)^{1 / 2} \\
\int_{0}^{L} F^{2} d x-T_{h}\left(F^{2}, 0, L\right) & \leq \frac{h^{2}}{4}\left(\int_{0}^{L} F_{x x}^{2} d x\right)^{1 / 2}\left(\int_{0}^{L} F^{2} d x\right)^{1 / 2} \\
\int_{0}^{M} \frac{F_{x}^{2}}{x^{2}} d x-T_{h}\left(\frac{F_{x}^{2}}{x^{2}}, 0, M\right) & \leq h\left(\int_{0}^{M} \frac{F_{x x}^{2}}{x^{2}} d x\right)^{1 / 2}\left(\int_{0}^{M} \frac{F_{x}^{2}}{x^{2}}\right)^{1 / 2} \\
\int_{0}^{L} F_{x}^{2} d x-T_{h}\left(F_{x}^{2}, 0, L\right) & \leq h\left(\int_{0}^{L} F_{x x}^{2} d x\right)^{1 / 2}\left(\int_{0}^{L} F_{x}^{2} d x\right)^{1 / 2}
\end{aligned}
$$

where $M=5, L=10, F$ is short for $F(\bar{\omega})$ and the Trapezoidal rule $T_{h}$ is given by

$$
T_{h}(f, a, b) \triangleq \sum_{a \leq i h<b} \frac{f(i h)+f((i+1) h)}{2} h .
$$

Since the approximate profile $\bar{\omega}$ is a piecewise cubic polynomial in $[0, L], \bar{\omega}$ is only in the class $C^{2,1}$ and $\bar{u}_{x x}$ blows up at $x=L$. Thus, the weight is singular and we cannot apply the standard error estimate for the Trapezoidal rule. From Lemma 3.1, for sufficiently small $h$, we can verify that $\left\langle F(\bar{\omega})^{2}, \varphi\right\rangle^{1 / 2},\left\langle(F(\bar{\omega}))_{x}^{2}, \psi\right\rangle^{1 / 2}$ are small using only the values of $F$ on the grid points and the Trapezoidal rule.

Proof. We first recall the standard error estimate for the Trapezoidal rule:

$$
\int_{a}^{b} f(x) d x-\frac{b-a}{2}(f(a)+f(b))=\int_{a}^{b} f^{\prime \prime}(x) \frac{(x-a)(x-b)}{2} d x .
$$

Denote by $P$ a piecewise quadratic polynomial

$$
P(x) \triangleq \frac{(x-i h)(x-(i+1) h)}{2}, \forall x \in[i h,(i+1) h] .
$$


Then we have

$$
\begin{aligned}
\Delta_{1} & \triangleq \int_{0}^{M} \frac{F^{2}}{x^{4}} d x-T_{h}\left(\frac{F^{2}}{x^{4}}, 0, M\right)=\int_{0}^{M}\left(\frac{F^{2}}{x^{4}}\right)_{x x} P(x) d x, \\
\left(\frac{F^{2}}{x^{4}}\right)_{x x} & =\frac{2 F_{x x} F+2 F_{x}^{2}}{x^{4}}-16 \frac{F_{x} F}{x^{5}}+20 \frac{F^{2}}{x^{6}} \\
& =2\left(\frac{F_{x x}}{x}-3 \frac{F_{x}}{x^{2}}\right) \frac{F}{x^{3}}+2 \frac{F_{x}^{2}}{x^{4}}-10 \frac{F_{x} F}{x^{5}}+20 \frac{F^{2}}{x^{6}} \geq 2\left(\frac{F_{x x}}{x}-3 \frac{F_{x}}{x^{2}}\right) \frac{F}{x^{3}},
\end{aligned}
$$

where we have used $2 a^{2}+20 b^{2} \geq 2 \sqrt{40}|a b| \geq 10 a b$. From (3.6), we know for $x \in[i h,(i+1) h]$

$$
P(x) \leq 0, \quad|P(x)| \leq \frac{1}{2}\left(\frac{(i+1) h-x+x-i h}{2}\right)^{2} \leq \frac{h^{2}}{8} .
$$

Combining the above estimates yields

$$
\begin{aligned}
\Delta_{1} & \leq \int_{0}^{M} 2\left(\frac{F_{x x}}{x}-3 \frac{F_{x}}{x^{2}}\right) \frac{F}{x^{3}} P(x) d x \leq \frac{h^{2}}{4} \int_{0}^{M}\left|\left(\frac{F_{x x}}{x}-3 \frac{F_{x}}{x^{2}}\right) \frac{F}{x^{3}}\right| d x \\
& \leq \frac{h^{2}}{4}\left(\int_{0}^{M}\left(\frac{F_{x x}}{x}-3 \frac{F_{x}}{x^{2}}\right)^{2} d x\right)^{1 / 2}\left(\int_{0}^{M} \frac{F^{2}}{x^{6}} d x\right)^{1 / 2} .
\end{aligned}
$$

Using integration by parts yields

$$
\begin{aligned}
& \int_{0}^{M}\left(\frac{F_{x x}}{x}-3 \frac{F_{x}}{x^{2}}\right)^{2} d x=\int_{0}^{M} \frac{F_{x x}^{2}}{x^{2}}+9 \frac{F_{x}^{2}}{x^{4}} d x-\int_{0}^{M} \frac{3}{x^{3}} d F_{x}^{2} \\
& =\int_{0}^{M} \frac{F_{x x}^{2}}{x^{2}}+9 \frac{F_{x}^{2}}{x^{4}} d x-9 \int_{0}^{M} \frac{F_{x}^{2}}{x^{4}} d x-\left.\frac{4 F_{x}^{2}}{x^{3}}\right|_{0} ^{M}=\int_{0}^{M} \frac{F_{x x}^{2}}{x^{2}}-\frac{4 F_{x}^{2}(M)}{M^{3}} \leq \int_{0}^{M} \frac{F_{x x}^{2}}{x^{2}},
\end{aligned}
$$

where we have used the regularity of the profile which satisfies

$$
\lim _{x \rightarrow 0+} \frac{F_{x}^{2}}{x^{3}}=0 .
$$

Combining (3.7) and (3.8) completes the proof of (3.1).

For (3.2), we introduce $G(x) \triangleq F(L-x)$. After a change of variables, (3.2) is equivalent to

$$
\int_{0}^{M} \frac{G^{2}}{x^{2}} d x-T_{h}\left(\frac{G^{2}}{x^{2}}, 0, M\right) \leq \frac{h^{2}}{4}\left(\int_{0}^{M} G_{x x} d x\right)^{1 / 2}\left(\int_{0}^{M} \frac{G^{2}}{x^{4}} d x\right)^{1 / 2} .
$$

The proof is very similar to the proof of (3.1) and is omitted. The proof of (3.3) is similar to the previous proof and is omitted here. The proof of (3.4) is based on the following expression of the error term

$$
\int_{a}^{b} f(x) d x=\frac{b-a}{2}(f(a)+f(b))-\int_{a}^{b} f^{\prime}(x)\left(x-\frac{a+b}{2}\right) d x .
$$

Denote by $Q(x)$ a piecewise linear function

$$
Q(x)=\frac{i h+(i+1) h}{2}-x, \quad x \in[i h,(i+1) h), \quad|Q(x)| \leq \frac{h}{2} .
$$

It follows that the left hand side of (3.4) can be written as

$$
\begin{aligned}
\Delta_{3} & =\int_{0}^{M}\left(\frac{F_{x}^{2}}{x^{2}}\right)_{x} Q(x) d x=2 \int_{0}^{M}\left(\frac{F_{x x}}{x}-\frac{F_{x}}{x^{2}}\right) \frac{F_{x}}{x} Q(x) d x \\
& \leq 2 \cdot \frac{h}{2} \int_{0}^{M}\left|\left(\frac{F_{x x}}{x}-\frac{F_{x}}{x^{2}}\right) \frac{F_{x}}{x}\right| d x \leq h\left(\int_{0}^{M}\left(\frac{F_{x x}}{x}-\frac{F_{x}}{x^{2}}\right)^{2} d x\right)^{1 / 2}\left(\int_{0}^{M} \frac{F_{x}^{2}}{x^{2}}\right)^{1 / 2} \\
& \leq h\left(\int_{0}^{M}\left(\frac{F_{x x}}{x}\right)^{2} d x\right)^{1 / 2}\left(\int_{0}^{M} \frac{F_{x}^{2}}{x^{2}}\right)^{1 / 2},
\end{aligned}
$$


where we have used integration by parts similar to (3.8) to get the last inequality. The proof of (3.5) is similar to (3.4) and is omitted.

A corollary of Lemma 3.1 is the following estimates of the error of the approximate profile.

Corollary 3.2. The error of approximate profile $F(\bar{\omega})$ satisfies

$$
\begin{aligned}
& \int_{0}^{M} \frac{F^{2}}{x^{4}} d x \leq T_{h}\left(\frac{F^{2}}{x^{4}}, 0, M\right)+\frac{B_{1}^{2}}{15} h^{2}, \int_{M}^{L} \frac{F^{2}}{(x-L)^{2}} d x \leq T_{h}\left(\frac{F^{2}}{(x-L)^{2}}, M, L\right)+\frac{B_{2}^{2}}{3} h^{2}, \\
& \int_{0}^{L} F^{2} d x \leq \frac{1}{4}\left(\frac{h^{2}}{4} B_{3}+\sqrt{\frac{h^{4}}{16} B_{3}^{2}+4 T_{h}\left(F^{2}, 0, L\right)}\right)^{2}, \\
& \int_{0}^{M} \frac{F_{x}^{2}}{x^{2}} d x \leq \frac{1}{4}\left(h B_{1}+\sqrt{h^{2} B_{1}^{2}+4 T_{h}\left(\frac{F_{x}^{2}}{x^{2}}, 0, M\right)}\right)^{2}, \\
& \int_{0}^{L} F_{x}^{2} d x \leq \frac{1}{4}\left(h B_{3}+\sqrt{h^{2} B_{3}^{2}+4 T_{h}\left(F_{x}^{2}, 0, L\right)}\right)^{2},
\end{aligned}
$$

where $B_{i}$ defined in (3.12) only depends on $K_{i}, J_{i}$ in (1.6).

Corollary 3.2 shows that the weighted $L^{2}, H^{1}$ errors of the approximate profile can be bounded by approximating the integral using the Trapezoidal rule with an error of order $O\left(h^{2}\right)$.

Proof. Using the Hardy inequality (the first inequality in (A.7) with $u-u_{x}(0) x$ replaced by $F$ ), we have

$$
\begin{gathered}
\int_{0}^{M} \frac{F^{2}}{x^{6}} d x \leq \frac{4}{25} \int_{0}^{M} \frac{F_{x}^{2}}{x^{4}} d x \leq \frac{4}{25} \cdot \frac{4}{9} \int_{0}^{M} \frac{F_{x x}^{2}}{x^{2}} d x=\left(\frac{4}{15}\right)^{2} \int_{0}^{M} \frac{F_{x x}^{2}}{x^{2}} d x, \\
\int_{M}^{L} \frac{F^{2}}{(x-L)^{4}} d x \leq \frac{4}{9} \int_{M}^{L} \frac{F_{x}^{2}}{(x-L)^{2}} \leq \frac{4}{9} \cdot 4 \int_{M}^{L} F_{x x}^{2} d x=\frac{16}{9} \int_{M}^{L} F_{x x}^{2} d x .
\end{gathered}
$$

Next, we estimate the weighted $L^{2}$ integral of $F_{x x}$. Note that

$$
F_{x x}=(F(\bar{\omega}))_{x x}=\bar{u}_{x x x} \bar{\omega}+\bar{u}_{x x} \bar{\omega}_{x}-\left(\bar{c}_{l} x+\bar{u}\right) \bar{\omega}_{x x x}-\left(\bar{c}_{l}+\bar{u}_{x}\right) \bar{\omega}_{x x} .
$$

We use different estimates for $F_{x x}$ :

$$
\begin{aligned}
& \frac{F_{x x}}{x}=\bar{u}_{x x x}\left(x^{2}-L^{2}\right) \frac{\bar{\omega}}{x\left(x^{2}-L^{2}\right)}+\frac{\bar{u}_{x x}}{x} \bar{\omega}_{x}-\frac{\overline{c_{l}} x+\bar{u}}{x} \bar{\omega}_{x x x}-\left(\bar{c}_{l}+\bar{u}_{x}\right) \frac{\bar{\omega}_{x x}}{x}, x \in[0, M], \\
& F_{x x}=\bar{u}_{x x x}\left(x^{2}-L^{2}\right) \frac{\bar{\omega}}{x^{2}-L^{2}}+\bar{u}_{x x} \bar{\omega}_{x}-\frac{\bar{c}_{l} x+\bar{u}}{x \wedge(L-x)}(x \wedge(L-x)) \bar{\omega}_{x x x}-\left(\bar{c}_{l}+\bar{u}_{x}\right) \bar{\omega}_{x x}, x \in[0, L] .
\end{aligned}
$$

For the terms involving $\bar{u}_{x x}, \bar{u}_{x x x}$, we use A.4 and A.5 in Lemma A.2 and the $L^{2}$ isometry of the Hilbert transform $H$

$$
\begin{aligned}
& \left\|\bar{u}_{x x x}\left(x^{2}-L^{2}\right)\right\|_{L^{2}[0, M]} \leq\left\|\bar{u}_{x x x}\left(x^{2}-L^{2}\right)\right\|_{L^{2}[0, L]}=\left\|\bar{\omega}_{x x}\left(x^{2}-L^{2}\right)\right\|_{L^{2}[0, L]}=J_{4}^{1 / 2}, \\
& \left\|\frac{\bar{u}_{x x}}{x}\right\|_{L^{2}[0, M]} \leq\left\|\frac{\bar{\omega}_{x}-\bar{\omega}_{x}(0)}{x}\right\|_{L^{2}[0, L]}=J_{3}^{1 / 2}, \quad\left\|\bar{u}_{x x}\right\|_{L^{2}[0, L]}=\left\|\bar{\omega}_{x}\right\|_{L^{2}[0, L]}=J_{6}^{1 / 2} .
\end{aligned}
$$

Note that $\bar{c}_{l} x+u(x)=0$ at $x=0, \pm L$. For the terms involving $\bar{u}, \bar{u}_{x}$, we use the $L^{\infty}$ estimate

$$
\max _{[0, L]}\left|\frac{\bar{c}_{l} x+\bar{u}}{x \wedge(L-x)}\right|=\max \left(\max _{[0, M]}\left|\frac{\bar{c}_{l} x+\bar{u}}{x}\right|, \max _{[M, L]}\left|\frac{\bar{c}_{l} x+\bar{u}}{x-L}\right|\right) \leq \max _{[0, L]}\left|\bar{c}_{l}+\bar{u}_{x}\right|=K_{3} .
$$

For the terms involving $\bar{\omega}, \bar{\omega}_{x}$, we also use the $L^{\infty}$ estimate

$$
\begin{gathered}
\left\|\frac{\bar{\omega}}{x\left(x^{2}-L^{2}\right)}\right\|_{L^{\infty}[0, M]}=K_{5 l}, \quad\left\|\frac{\bar{\omega}}{\left(x^{2}-L^{2}\right)}\right\|_{L^{\infty}[0, L]}=K_{5}, \\
\left\|\bar{\omega}_{x}\right\|_{L^{\infty}[0, L]}=K_{1}, \quad\left\|\bar{\omega}_{x}\right\|_{L^{\infty}[M, L]}=K_{1 r} .
\end{gathered}
$$


For the terms involving $\bar{\omega}_{x x}, \bar{\omega}_{x x x}$, we use the $L^{2}$ estimate

$$
\begin{aligned}
& \left\|\bar{\omega}_{x x x}\right\|_{L^{2}[0, M]}=J_{1}^{1 / 2}, \quad\left\|\frac{\bar{\omega}_{x x}}{x}\right\|_{L^{2}[0, M]}=J_{2}^{1 / 2}, \quad\left\|\bar{\omega}_{x x x}^{2}(x \wedge(L-x))\right\|_{L^{2}[0, L]}=J_{5}^{1 / 2}, \\
& \left\|\bar{\omega}_{x x x}^{2}(x \wedge(L-x))\right\|_{L^{2}[M, L]}=J_{5 r}^{1 / 2}, \quad\left\|\bar{\omega}_{x x}\right\|_{L^{2}[0, L]}=J_{7}^{1 / 2}, \quad\left\|\bar{\omega}_{x x}\right\|_{L^{2}[M, L]}=J_{7 r}^{1 / 2} .
\end{aligned}
$$

We apply the above estimates and the triangle inequality to yield

$$
\begin{gathered}
\left\|\frac{F_{x x}}{x}\right\|_{L^{2}[0, M]} \leq K_{5 l} J_{4}^{1 / 2}+K_{1} J_{3}^{1 / 2}+K_{3}\left(J_{1}^{1 / 2}+J_{2}^{1 / 2}\right) \triangleq B_{1}, \\
\left\|F_{x x}\right\|_{L^{2}[M, L]} \leq K_{5} J_{4}^{1 / 2}+K_{1 r} J_{6}^{1 / 2}+K_{3}\left(J_{5 r}^{1 / 2}+J_{7 r}^{1 / 2}\right) \triangleq B_{2}, \\
\left\|F_{x x}\right\|_{L^{2}[0, L]} \leq K_{5} J_{4}^{1 / 2}+K_{1} J_{6}^{1 / 2}+K_{3}\left(J_{5}^{1 / 2}+J_{7}^{1 / 2}\right) \triangleq B_{3} .
\end{gathered}
$$

Combining (3.1), (3.2) in Lemma 3.1 the Hardy inequality (3.11) and the above estimates (3.12), we obtain the first two inequalities in (3.9).

$$
\begin{aligned}
\int_{0}^{M} \frac{F^{2}}{x^{4}} d x-T_{h}\left(\frac{F^{2}}{x^{4}}, 0, M\right) & \leq \frac{h^{2}}{15} \int_{0}^{M} \frac{F_{x x}^{2}}{x^{2}} d x \leq \frac{B_{1}^{2}}{15} h^{2}, \\
\int_{M}^{L} \frac{F^{2}}{(x-L)^{2}} d x-T_{h}\left(\frac{F^{2}}{(x-L)^{2}}, M, L\right) & \leq \frac{h^{2}}{3} \int_{M}^{L} F_{x x}^{2} d x \leq \frac{B_{2}^{2}}{3} h^{2} .
\end{aligned}
$$

To obtain the remaining inequalities in (3.9), we note that (3.3), (3.4) and (3.5) are quadratic inequalities with respect to

$$
I_{1} \triangleq\|F\|_{L^{2}[0, L]}, \quad I_{2} \triangleq\left\|\frac{F_{x}}{x}\right\|_{L^{2}[M, L]}, \quad I_{3} \triangleq\left\|F_{x}^{2}\right\|_{L^{2}[0, L]},
$$

and the coefficients on the right hand side of (3.3), (3.4) and (3.5) are bounded by $B_{i}$ defined in (3.12). Using (3.3) and (3.12), we get

$$
I_{1}^{2} \leq T_{h}\left(F^{2}, 0, L\right)+\frac{h^{2}}{4} B_{3} I_{1},
$$

from which we can solve $I_{1}$ and obtain the following estimate

$$
I_{1} \leq \frac{1}{2}\left(\frac{h^{2}}{4} B_{3}+\sqrt{\frac{h^{4}}{16} B_{3}^{2}+4 T_{h}\left(F^{2}, 0, L\right)}\right) .
$$

Taking square on both sides, we prove the third inequality in (3.9). The bound for $I_{2}, I_{3}$ can be obtained similarly and we omit their proofs.

Using (3.12) and the rigorous bounds in (1.7), we have that

$$
B_{1}<2.217, \quad B_{2}<11.05, \quad B_{3}<12.16 .
$$

We will use the interval arithmetics computation to verify that

$$
\begin{aligned}
& T_{h}\left(F^{2}, 0, L\right)<8.445 \times 10^{-10}, \quad T_{h}\left(\frac{F^{2}}{x^{4}}, 0, M\right)<7.388 \times 10^{-10} . \\
& T_{h}\left(\frac{F^{2}}{(x-L)^{2}}, M, L\right)<6.248 \times 10^{-9} \\
& T_{h}\left(F_{x}^{2}, 0, L\right)<9.850 \times 10^{-9}, \quad T_{h}\left(\frac{F_{x}^{2}}{x^{2}}, 0, M\right)<2.197 \times 10^{-9} .
\end{aligned}
$$


Then using (3.9), we obtain the following rigorous upper bounds:

$$
\begin{aligned}
& I F_{1} \triangleq \int_{0}^{M} \frac{F^{2}}{x^{4}} d x<9.435 \times 10^{-10}<10^{-9} \\
& I F_{2} \triangleq \int_{M}^{L} \frac{F^{2}}{(x-L)^{2}} d x<3.164 \times 10^{-8}<4 \times 10^{-8}, \\
& I F_{3} \triangleq \int_{0}^{L} F^{2} d x<8.446 \times 10^{-10}<10^{-9} \\
& I F_{4} \triangleq \int_{0}^{M} \frac{F_{x}^{2}}{x^{2}} d x<6.749 \times 10^{-9}<10^{-8} \\
& I F_{5} \triangleq \int_{0}^{L} F_{x}^{2} d x<1.112 \times 10^{-7}<2 \times 10^{-7} .
\end{aligned}
$$

3.2. Estimate of the error terms. We choose the functions $\rho_{1}, \rho_{2}$ as follows

$$
\rho_{1}=1.25 x^{-4} \mathbf{1}_{x \leq M}+0.1+0.01(x-L)^{-2} \mathbf{1}_{x \geq M}, \quad \rho_{2}=x^{-2} \mathbf{1}_{x \leq M}+0.02 .
$$

Using these weights and the Cauchy-Schwartz inequality, we can estimate the error terms in the weighted $L^{2}, H^{1}$ errors (1.4) as follows

$$
\begin{gathered}
\left|F_{1}\right|=|\langle F(\bar{\omega}), \omega \varphi\rangle| \leq \frac{1}{4 \tau}\left\langle F(\bar{\omega})^{2}, \rho_{1}\right\rangle+\tau\left\langle\omega^{2}, \varphi^{2} \rho_{1}^{-1}\right\rangle, \\
\left|F_{2}\right|=\left|\left\langle(F(\bar{\omega}))_{x}, \omega \varphi\right\rangle\right| \leq \frac{1}{4 \tau}\left\langle(F(\bar{\omega}))_{x}^{2}, \rho_{2}\right\rangle+\tau\left\langle\omega_{x}^{2}, \psi^{2} \rho_{2}^{-1}\right\rangle,
\end{gathered}
$$

where $\tau$ is to be determined. Choose $\mu=0.02$ and define $F_{0}$ below

$$
F_{0} \triangleq \frac{1}{4 \tau}\left\langle F(\bar{\omega})^{2}, \rho_{1}\right\rangle+\frac{\mu}{4 \tau}\left\langle(F(\bar{\omega}))_{x}^{2}, \rho_{2}\right\rangle
$$

We then obtain

$$
\left|F_{1}+\mu F_{2}\right| \leq F_{0}+\tau\left\langle\omega^{2}, \varphi^{2} \rho_{1}^{-1}\right\rangle+\mu \tau\left\langle\omega_{x}^{2}, \psi^{2} \rho_{2}^{-1}\right\rangle .
$$

Using the upper bounds of the integrals in (3.13) and the definition of $\rho_{i}$ in (3.14), we get

$$
F_{0} \leq \frac{1}{4 \tau}\left(1.25 \cdot I F_{1}+0.01 \cdot I F_{2}+0.1 \cdot I F_{3}+5 \mu \cdot I F_{4}+0.1 \mu \cdot I F_{5}\right) \leq \frac{1}{4 \tau} \cdot 1.760 \cdot 10^{-9}
$$

\section{Estimate of the NONLINEAR TERM}

4.1. Estimate of $u_{x}, \omega, u / x$. To control the nonlinear term, it suffices to control $\left\|u_{x}\right\|_{L^{\infty}[0, L]}$. First of all, we have the following comparison result.

Lemma 4.1. The weights $\varphi, \psi$ satisfy for $x \in[0, L]$

$$
\begin{aligned}
& \varphi(x) \geq 1.15\left(\frac{1}{x^{4}}+\frac{0.02}{x^{2}}\right) \triangleq \varphi_{1}(x), \varphi(x) \geq 0.0085\left(\frac{1}{(x-L)^{2}}+\frac{1}{(x+L)^{2}}\right) \triangleq \varphi_{2}(x), \\
& \psi(x) \geq 0.97\left(x^{-2}+0.01\right) \triangleq \psi_{1}(x) .
\end{aligned}
$$

Lemma A.2 and the $L^{2}$ isometry of the Hilbert transform (A.7) with $p=2,4$ then imply

$$
\begin{aligned}
& \left\langle\omega^{2}, \varphi\right\rangle \geq\left\langle\omega^{2}, \varphi_{1}\right\rangle=\int_{0}^{L}\left(u_{x}-u_{x}(0)\right)^{2} \varphi_{1} d x, \\
& \left\langle\omega^{2}, \varphi\right\rangle \geq\left\langle\omega^{2}, \varphi_{2}\right\rangle=\int_{0}^{L}\left(u_{x}-u_{x}(L)\right)^{2} \varphi_{2} d x, \quad\left\langle\omega_{x}^{2}, \psi\right\rangle \geq\left\langle\omega_{x}^{2}, \psi_{1}\right\rangle=\int_{0}^{L} u_{x x}^{2} \psi_{1} d x .
\end{aligned}
$$

We plot the numerical values of $\varphi_{1} / \varphi, \varphi_{2} / \varphi, \psi_{1} / \psi$ on the grid points in the first subfigure in Figure 2 in the Appendix.

We consider the following functions and energy

$$
\xi_{1} \triangleq x^{-3}+0.0125 x^{-1}, \quad \xi_{2} \triangleq(L-x)^{-1}+0.029(L-x), \quad E^{2}(t) \triangleq\left\langle\omega^{2}, \varphi\right\rangle+\mu\left\langle\omega_{x}^{2}, \psi\right\rangle,
$$


where $\mu>0$ are to be determined. For $x<0, \xi_{2}$ should be considered as $\xi_{2}=(L-|x|)^{-1}+$ $0.029(L-|x|)$. Due to the odd/even symmetry, we only focus on $x>0$ and drop the absolute sign to simplify the notations. We have the following estimate for $u_{x}$.

Lemma 4.2. Suppose that $\alpha_{1}, \alpha_{2}$ satisfies

$$
\xi_{i}^{2} \leq \mu\left(\alpha_{i} \varphi_{i}-\xi_{i, x}\right) \alpha_{i} \psi_{i}, i=1,2,
$$

where $\psi_{2}=\psi_{1}$ and $\varphi_{i}$ are defined in Lemma 4.1. Then we have

$$
\left|u_{x}(x)-u_{x}(0)\right| \leq\left(\frac{\alpha_{1}}{\xi_{1}(x)}\right)^{1 / 2} E(t), \quad\left|u_{x}(x)-u_{x}(L)\right| \leq\left(\frac{\alpha_{2}}{\xi_{2}(x)}\right)^{1 / 2} E(t) .
$$

By definition, $\xi_{1}(x)>0$ and $\xi_{2}(x)>0$ for $x \in[0, L]$. We will choose $\alpha_{1}=5.6$ and $\alpha_{2}=500$. For these parameters, we plot the numerical values of the ratio between the left and the right hand sides of (4.4) on the grid points in the second subfigure in Figure 2 in the Appendix.

Proof. Note that $\left(u_{x}-u_{x}(0)\right)^{2} \xi_{1}$ vanishes at $x=0$ due to $u_{x}-u_{x}(0)=O\left(x^{2}\right)$. For $x$ close to 0 , we differentiate it and use the Cauchy-Schwartz inequality to yield

$$
\begin{aligned}
& \left(u_{x}-u_{x}(0)\right)^{2} \xi_{1}=\int_{0}^{x} 2 u_{x x}\left(u_{x}-u_{x}(0)\right) \xi_{1} d x+\int_{0}^{x}\left(u_{x}-u_{x}(0)\right)^{2} \xi_{1, x} d x \\
\leq & \int_{0}^{x}\left(u_{x}-u_{x}(0)\right)^{2}\left(\alpha_{1} \varphi_{1}-\xi_{1, x}\right)+u_{x x}^{2} \xi_{1}^{2}\left(\alpha_{1} \varphi_{1}-\xi_{1, x}\right)^{-1} d x+\int_{0}^{x}\left(u_{x}-u_{x}(0)\right)^{2} \xi_{1, x} d x \\
= & \int_{0}^{x}\left(u_{x}-u_{x}(0)\right)^{2}\left(\alpha_{1} \varphi_{1}\right) d x+\int_{0}^{x} u_{x x}^{2} \xi_{1}^{2}\left(\alpha_{1} \varphi_{1}-\xi_{1, x}\right)^{-1} d x,
\end{aligned}
$$

where we have used $\alpha_{1} \varphi_{1}-\xi_{1, x}>0$ (4.4) when we applied the Cauchy-Schwartz inequality. Using the assumption (4.4), we obtain

$$
\left(u_{x}-u_{x}(0)\right)^{2} \xi_{1} \leq \int_{0}^{x}\left(u_{x}-u_{x}(0)\right)^{2}\left(\alpha_{1} \varphi_{1}\right) d x+\int_{0}^{x} u_{x x}^{2} \mu \alpha_{1} \psi_{1} d x .
$$

Combining the above estimates and (4.2), we prove

$$
\left(u_{x}-u_{x}(0)\right)^{2} \xi_{1} \leq \alpha_{1}\left\langle\omega^{2}, \varphi\right\rangle+\mu \alpha_{1}\left\langle\omega_{x}^{2}, \psi\right\rangle=\alpha_{1} E^{2}(t) .
$$

Taking the square root yields the first estimate in (4.5). For $x$ close to $L$, applying an argument similar to that in our estimate for $\left(u_{x}-u_{x}(L)\right)^{2} \xi_{2}$ yields

$$
\left|u_{x}(x)-u_{x}(L)\right| \leq\left(\alpha_{2} \xi_{2}^{-1}\right)^{1 / 2} E(t),
$$

which is the second estimate in (4.5).

For $\omega$, we have a similar result.

Lemma 4.3. Suppose that the assumptions in Lemma 4.2 holds and $x>0$. We have

$$
|\omega(x)| \leq\left(\frac{\xi_{1}(x)}{\alpha_{1}}+\frac{\xi_{2}(x)}{\alpha_{2}}\right)^{-1 / 2} E(t) .
$$

Proof. Using an estimate similar to that in the proof of Lemma 4.2, we have

$$
\omega^{2}(x) \xi_{1}(x) \leq \alpha_{1}\left(\int_{0}^{x} \omega^{2} \varphi_{1} d x+\mu \int_{0}^{x} \omega_{x}^{2} \psi_{1} d x\right), \omega^{2}(x) \xi_{2}(x) \leq \alpha_{2}\left(\int_{x}^{L} \omega^{2} \varphi_{2} d x+\mu \int_{x}^{L} \omega_{x}^{2} \psi_{1} d x\right) .
$$

Using (4.1) and the above estimate, we derive

$$
\begin{aligned}
\omega^{2}(x)\left(\frac{\xi_{1}(x)}{\alpha_{1}}+\frac{\xi_{2}(x)}{\alpha_{2}}\right) & \leq\left(\int_{0}^{x} \omega^{2} \varphi d x+\mu \int_{0}^{x} \omega_{x}^{2} \psi d x\right)+\left(\int_{x}^{L} \omega^{2} \varphi d x+\mu \int_{x}^{L} \omega_{x}^{2} \psi d x\right) \\
& =\left\langle\omega^{2}, \varphi\right\rangle+\mu\left\langle\omega_{x}^{2}, \psi\right\rangle=E^{2}(t),
\end{aligned}
$$

which further implies (4.6) after taking the square root. 
A direct result of Lemma 4.2 is the following lemma.

Lemma 4.4. Suppose that the assumption in Lemma 4.2 holds and $x>0$. We have

$$
\left|\frac{u-u_{x}(0) x}{x}\right| \leq \frac{2}{5} \alpha_{1}^{1 / 2} x^{3 / 2} E(t),\left|\frac{u-u(L)-u_{x}(L)(x-L)}{x-L}\right| \leq \frac{2}{3} \alpha_{2}^{1 / 2}(L-x)^{1 / 2} E(t) .
$$

Proof. From the definition of $\xi_{1}, \xi_{2}$ in (4.3), we know

$$
\left(\frac{\alpha_{1}}{\xi_{1}}\right)^{1 / 2}=\left(\frac{\alpha_{1} x^{3}}{1+0.0125 x^{2}}\right)^{1 / 2} \leq \alpha_{1}^{1 / 2} x^{3 / 2}, \quad\left(\frac{\alpha_{2}}{\xi_{2}}\right)^{1 / 2}=\left(\frac{\alpha_{2}(L-x)}{1+0.029(L-x)^{2}}\right)^{1 / 2} \leq \alpha_{2}^{1 / 2}(L-x)^{1 / 2} .
$$

Applying the above estimate and (4.5), we yield

$$
\begin{aligned}
\left|\frac{u-u_{x}(0) x}{x}\right| & =\left|\frac{1}{x} \int_{0}^{x} u_{x}(y)-u_{x}(0) d y\right| \leq \frac{1}{x} \int_{0}^{x}\left(\frac{\alpha_{1}}{\xi_{1}}\right)^{1 / 2} d y \cdot E(t) \\
& \leq \frac{\alpha^{1 / 2}}{x} E(t) \int_{0}^{x} y^{3 / 2} d y=\frac{2}{5} \alpha_{1}^{1 / 2} x^{3 / 2} E(t)
\end{aligned}
$$

which is the first inequality in (4.7). Using (4.8) and (4.5), one can derive the second inequality in (4.7) similarly. The factor $2 / 3 \cdot(L-x)^{1 / 2}$ comes from

$$
\frac{1}{L-x} \int_{x}^{L}(L-y)^{1 / 2} d y=\frac{2}{3}(L-x)^{1 / 2}
$$

For the end points $u_{x}(0), u_{x}(L), c_{\omega}=-u(L) / L$, we use (1.5) and the Cauchy-Schwartz inequality to obtain the following estimate

$$
\begin{aligned}
& \left|c_{\omega}+u_{x}(0)\right|=\left|\left\langle g_{c_{\omega}}+g_{u_{x}(0)}, \omega\right\rangle\right| \leq\left\langle\omega^{2}, \varphi\right\rangle^{1 / 2}\left\langle\left(g_{c_{\omega}}+g_{u_{x}(0)}\right)^{2}, \varphi^{-1}\right\rangle^{1 / 2} \leq \gamma_{1} E(t), \\
& \left|c_{\omega}+u_{x}(L)\right|=\left|\left\langle g_{c_{\omega}}+g_{u_{x}(L)}, \omega\right\rangle\right| \leq\left\langle\omega^{2}, \varphi\right\rangle^{1 / 2}\left\langle\left(g_{c_{\omega}}+g_{u_{x}(L)}\right)^{2}, \varphi^{-1}\right\rangle^{1 / 2} \leq \gamma_{2} E(t),
\end{aligned}
$$

where we have used $\left\langle\omega^{2}, \varphi\right\rangle \leq E(t)$ and the constants $\gamma_{1}, \gamma_{2}$ are given by

$$
\gamma_{1} \triangleq\left\langle\left(g_{c_{\omega}}+g_{u_{x}(0)}\right)^{2}, \varphi^{-1}\right\rangle^{1 / 2}, \quad \gamma_{2} \triangleq\left\langle\left(g_{c_{\omega}}+g_{u_{x}(L)}\right)^{2}, \varphi^{-1}\right\rangle^{1 / 2} .
$$

4.2. Estimate of the nonlinear terms. Recall the nonlinear terms $N, N_{1}, N_{2}$ in (1.4) and the normalization conditions of $c_{l}, c_{\omega}(1.3)$. Using integration by part, we have

$$
\begin{aligned}
N_{1} & =\left\langle\left(c_{\omega}+u_{x}\right) \omega-\left(c_{l} x+u\right) \omega_{x}, \omega \varphi\right\rangle=\left\langle\frac{\left(\left(c_{l} x+u\right) \varphi\right)_{x}}{2 \varphi}+c_{\omega}+u_{x}, \omega^{2} \varphi\right\rangle \\
& =\left\langle\frac{3}{2}\left(c_{\omega}+u_{x}\right)+\left(c_{l}+\frac{u}{x}\right) \frac{x \varphi_{x}}{2 \varphi}, \omega^{2} \varphi\right\rangle \triangleq\left\langle T, \omega^{2} \varphi\right\rangle .
\end{aligned}
$$

We use different estimates to handle $T$ for $x$ close to 0 and $x$ close to $L$. For $x$ close to 0 , we have

$$
T=\left(c_{\omega}+u_{x}(0)\right)\left(\frac{3}{2}+\frac{x \varphi_{x}}{2 \varphi}\right)+\frac{3}{2}\left(u_{x}-u_{x}(0)\right)+\left(\frac{u}{x}-u_{x}(0)\right) \frac{x \varphi_{x}}{2 \varphi} .
$$

Using (4.5), 4.7) and (4.9), we obtain

$$
|T| \leq\left(\gamma_{1}\left|\frac{3}{2}+\frac{x \varphi_{x}}{2 \varphi}\right|+\frac{3}{2}\left(\frac{\alpha_{1}}{\xi_{1}}\right)^{1 / 2}+\frac{2}{5} \alpha_{1}^{1 / 2} x^{3 / 2}\left|\frac{x \varphi_{x}}{2 \varphi}\right|\right) E(t) .
$$

For $x$ close to $L$, we use another decomposition to handle $T$

$T=\left(c_{\omega}+u_{x}(L)\right)\left(\frac{3}{2}+\frac{(x-L) \varphi_{x}}{2 \varphi}\right)+\frac{3}{2}\left(u_{x}-u_{x}(L)\right)+\frac{u(x)-u(L)-u_{x}(L)(x-L)}{x-L} \frac{(x-L) \varphi_{x}}{2 \varphi}$.

Using (4.5), (4.7) and (4.9), we obtain

$$
|T| \leq\left(\gamma_{2}\left|\frac{3}{2}+\frac{(x-L) \varphi_{x}}{2 \varphi}\right|+\frac{3}{2}\left(\frac{\alpha_{2}}{\xi_{2}}\right)^{1 / 2}+\frac{2}{3} \alpha_{2}^{1 / 2}(L-x)^{1 / 2}\left|\frac{(x-L) \varphi_{x}}{2 \varphi}\right|\right) E(t) .
$$


Combining (4.10), (4.11) and (4.12), we obtain

$$
\left|N_{1}\right| \leq\left\langle Z_{1}(x), \omega^{2} \varphi\right\rangle E(t),
$$

where $Z_{1}(x)$ is given by

$$
\begin{aligned}
Z_{1}(x) \triangleq & \min \left(\gamma_{1}\left|\frac{3}{2}+\frac{x \varphi_{x}}{2 \varphi}\right|+\frac{3}{2}\left(\frac{\alpha_{1}}{\xi_{1}}\right)^{1 / 2}+\frac{2}{5} \alpha_{1}^{1 / 2} x^{3 / 2}\left|\frac{x \varphi_{x}}{2 \varphi}\right|\right. \\
& \left.\gamma_{2}\left|\frac{3}{2}+\frac{(x-L) \varphi_{x}}{2 \varphi}\right|+\frac{3}{2}\left(\frac{\alpha_{2}}{\xi_{2}}\right)^{1 / 2}+\frac{2}{3} \alpha_{2}^{1 / 2}(L-x)^{1 / 2}\left|\frac{(x-L) \varphi_{x}}{2 \varphi}\right|\right) .
\end{aligned}
$$

For $N_{2}$ in (1.4), we use integration by parts to obtain

$$
\begin{aligned}
N_{2} & =\left\langle\left(\left(c_{\omega}+u_{x}\right) \omega-\left(c_{l} x+u\right) \omega_{x}\right)_{x}, \omega_{x} \psi\right\rangle=\left\langle u_{x x} \omega-\left(c_{l} x+u\right) \omega_{x x}, \omega_{x} \psi\right\rangle \\
& =\left\langle\frac{\left(\left(c_{l} x+u\right) \psi\right)_{x}}{2 \psi}, \omega_{x}^{2} \psi\right\rangle+\left\langle u_{x x} \omega, \omega_{x} \psi\right\rangle \triangleq N_{2,1}+N_{2,2} .
\end{aligned}
$$

For the first part, using (4.10), (4.11), (4.12) and an estimate similar to that in our estimate for $N_{1}$, we obtain

$$
N_{2,1} \leq\left\langle Z_{2}(x), \omega_{x}^{2} \psi\right\rangle E(t)
$$

where $Z_{2}(x)$ is given by

$$
\begin{aligned}
Z_{2}(x) \triangleq & \min \left(\gamma_{1}\left|\frac{1}{2}+\frac{x \psi_{x}}{2 \psi}\right|+\frac{1}{2}\left(\frac{\alpha_{1}}{\xi_{1}}\right)^{1 / 2}+\frac{2}{5} \alpha_{1}^{1 / 2} x^{3 / 2}\left|\frac{x \psi_{x}}{2 \psi}\right|\right. \\
& \left.\gamma_{2}\left|\frac{1}{2}+\frac{(x-L) \psi_{x}}{2 \psi}\right|+\frac{1}{2}\left(\frac{\alpha_{2}}{\xi_{2}}\right)^{1 / 2}+\frac{2}{3} \alpha_{2}^{1 / 2}(L-x)^{1 / 2}\left|\frac{(x-L) \psi_{x}}{2 \psi}\right|\right) .
\end{aligned}
$$

For $N_{2,2}$, we use (4.1), (4.2) and the Cauchy-Schwartz inequality to yield

$$
\left|N_{2,2}\right| \leq\left\langle u_{x x}^{2}, \psi_{1}\right\rangle^{1 / 2}\left\langle\omega^{2}, \omega_{x}^{2} \psi^{2} \psi_{1}^{-1}\right\rangle^{1 / 2} \leq\left\langle\omega_{x}^{2}, \psi_{1}\right\rangle^{1 / 2}\left\langle\omega^{2}, \omega_{x}^{2} \psi^{2} \psi_{1}^{-1}\right\rangle^{1 / 2} .
$$

For some constant $b_{3}>0$ to be determined, we use the above estimate and (4.6) to derive (4.18)

$$
\begin{aligned}
& \left|N_{2,2}\right| \leq b_{3}\left\langle\omega_{x}^{2}, \psi_{1}\right\rangle E(t)+\frac{1}{4 b_{3}}\left\langle\omega^{2}, \omega_{x}^{2} \psi^{2} \psi_{1}^{-1}\right\rangle E(t)^{-1} \\
\leq & b_{3}\left\langle\omega_{x}^{2} \psi, \psi_{1} \psi^{-1}\right\rangle E(t)+\frac{1}{4 b_{3}}\left\langle\left(\frac{\xi_{1}(x)}{\alpha_{1}}+\frac{\xi_{2}(x)}{\alpha_{2}}\right)^{-1}, \omega_{x}^{2} \psi^{2} \psi_{1}^{-1}\right\rangle E(t) \triangleq\left\langle Z_{3}(x), \omega_{x}^{2} \psi\right\rangle E(t),
\end{aligned}
$$

where $Z_{3}(x)$ is given by

$$
Z_{3}(x)=b_{3} \psi_{1} \psi^{-1}+\frac{1}{4 b_{3}}\left(\frac{\xi_{1}(x)}{\alpha_{1}}+\frac{\xi_{2}(x)}{\alpha_{2}}\right)^{-1} \psi \psi_{1}^{-1} .
$$

We choose $b_{3}=10$ in the final estimate.

4.3. Summary of the estimates for the nonlinear terms. Combining the estimate (4.13), (4.15), (4.16) and (4.18), we prove

(4.20) $\left|N_{1}\right|+\mu\left|N_{2}\right| \leq\left|N_{1}\right|+\mu\left(\left|N_{2,1}\right|+\left|N_{2,2}\right|\right) \leq\left\langle Z_{1}(x), \omega^{2} \varphi\right\rangle E(t)+\mu\left\langle Z_{2}(x)+Z_{3}(x), \omega_{x}^{2} \psi\right\rangle E(t)$, where $Z_{1}, Z_{2}$ and $Z_{3}$ are defined in (4.14), (4.17) and (4.19), respectively.

\section{Nonlinear estimate}

In this section, we combine all the estimates to obtain the nonlinear stability. 
Optimizing the parameters. We choose the following parameter

$$
\mu=0.02, \quad \tau=0.05, \quad \alpha_{1}=5.6, \quad \alpha_{2}=500 .
$$

We can verify that $\alpha_{1}, \alpha_{2}$ given above satisfy the assumption in Lemma 4.2 .

Combining the weighted $L^{2}$ estimate

$$
\frac{1}{2} \frac{d}{d t}\left\langle\omega^{2}, \varphi\right\rangle \leq-0.3\left\langle\omega^{2}, \varphi\right\rangle+N_{1}+F_{1},
$$

the weighted $H^{1}$ estimate (2.4) in Lemma 2.1, the energy $E(t)$ in (4.3), the estimate of error term (3.15) and the estimate of nonlinear term (4.20), we have proved rigorously that

$$
\begin{aligned}
\frac{1}{2} \frac{d}{d t} E(t)^{2} & =\frac{1}{2} \frac{d}{d t}\left(\left\langle\omega^{2}, \varphi\right\rangle+\mu\left\langle\omega_{x}^{2}, \psi\right\rangle\right) \leq(-0.3+7.5 \mu)\left\langle\omega^{2}, \varphi\right\rangle-0.25 \mu\left\langle\omega_{x}^{2}, \psi\right\rangle \\
& +\left(F_{0}+\tau\left\langle\omega^{2}, \varphi^{2} \rho_{1}^{-1}\right\rangle+\mu \tau\left\langle\omega_{x}^{2}, \psi^{2} \rho_{2}^{-1}\right\rangle\right)+\left\langle Z_{1}(x), \omega^{2} \varphi\right\rangle E(t)+\mu\left\langle Z_{2}(x)+Z_{3}(x), \omega_{x}^{2} \psi\right\rangle E(t) . \\
= & -0.15\left\langle\omega^{2}, \varphi\right\rangle-0.25 \mu\left\langle\omega_{x}^{2}, \psi\right\rangle+F_{0}+\left\langle\tau \varphi \rho_{1}^{-1}+Z_{1}(x) E(t), \omega^{2} \varphi\right\rangle \\
& +\mu\left\langle\tau \psi \rho_{2}^{-1}+\left(Z_{2}(x)+Z_{3}(x)\right) E(t), \omega_{x}^{2} \psi\right\rangle,
\end{aligned}
$$

where we have used $\mu=0.02$ to obtain the last equality. We divide the damping term into two parts as follows to control the error and the nonlinear term

$$
-0.15\left\langle\omega^{2}, \varphi\right\rangle-0.25 \mu\left\langle\omega_{x}^{2}, \psi\right\rangle=-0.05 E(t)^{2}-0.1\left\langle\omega^{2}, \varphi\right\rangle-0.2 \mu\left\langle\omega_{x}^{2}, \psi\right\rangle .
$$

With this decomposition, we can further rewrite (5.1) as follows

$$
\begin{aligned}
\frac{1}{2} \frac{d}{d t} E(t)^{2} & \leq-0.05 E(t)^{2}+F_{0}+\left\langle-0.1+\tau \varphi \rho_{1}^{-1}+Z_{1}(x) E(t), \omega^{2} \varphi\right\rangle \\
& +\mu\left\langle-0.2+\tau \psi \rho_{2}^{-1}+\left(Z_{2}(x)+Z_{3}(x)\right) E(t), \omega_{x}^{2} \psi\right\rangle .
\end{aligned}
$$

We use the bootstrap argument to complete the proof. We choose the threshold below

$$
E^{*} \triangleq 5 \cdot 10^{-4} \text {. }
$$

Suppose that $E(0)<E^{*}$. To complete the bootstrap argument, it suffices to show that the right hand side of (5.2) is negative at $E(t)=E^{*}$. In particular, it can be verified rigorously that

$$
\left\{\begin{array} { l l } 
{ F _ { 0 } - 0 . 0 5 E ^ { * 2 } } & { < 0 } \\
{ - 0 . 1 + \tau \varphi \rho _ { 1 } ^ { - 1 } + Z _ { 1 } ( x ) E ^ { * } } & { < 0 } \\
{ - 0 . 2 + \tau \psi \rho _ { 2 } ^ { - 1 } + ( Z _ { 2 } ( x ) + Z _ { 3 } ( x ) ) E ^ { * } } & { < 0 }
\end{array} \Longleftrightarrow \left\{\begin{array}{ll}
\frac{1}{0.2} \cdot 1.389 \cdot 10^{-9}-0.05 E^{* 2} & <0 \\
-0.1+0.05 \varphi \rho_{1}^{-1}+Z_{1}(x) E^{*} & <0 \\
-0.2+0.05 \psi \rho_{2}^{-1}+\left(Z_{2}(x)+Z_{3}(x)\right) E^{*} & <0
\end{array}\right.\right.
$$

where we have used (3.16) and substituted $\tau=0.05$. The first inequality comes from a direct calculation. We plot the numerical values of the left hand side of the second and the third quantity on the grid points in Figure 3 in the Appendix. Therefore, we prove that the bootstrap argument can be continued. Hence, $E(t)<E^{*}$ for all $t>0$ and the nonlinear stability follows.

With this a-priori estimate, one can further establish the convergence result using an argument similar to that in the proof for small $|a|$.

\section{Appendix A. Some useful Lemmas}

The following Lemmas are proved in the Appendix of [1] and we have used them in this supplementary material.

Lemma A.1 (The Tricomi identity). We have

$$
H(\omega H \omega)=\frac{1}{2}\left((H \omega)^{2}-\omega^{2}\right) .
$$

Lemma A.2. Suppose that $u_{x}=H \omega$. Then we have

$$
\frac{u_{x}-u_{x}(0)}{x}=H\left(\frac{\omega}{x}\right) \text {, or equivalently }(H \omega)(x)=(H \omega)(0)+x H\left(\frac{\omega}{x}\right) \text {. }
$$


Similarly, we have

$$
\frac{u_{x}-u_{x}(L)}{x-L}=H\left(\frac{\omega}{x-L}\right), u_{x x}=H \omega_{x}, x u_{x x}=H\left(x \omega_{x}\right)
$$

Suppose that in addition $\omega$ is odd. Then we have

$$
x^{2} u_{x x}=H\left(x^{2} \omega_{x}\right), \quad x u_{x}=H(x \omega), \quad \frac{u_{x x}}{x}=H\left(\frac{\omega_{x}-\omega_{x}(0)}{x}\right) .
$$

If $\omega$ is odd and a piecewise cubic polynomial supported on $[-L, L]$ with $\omega(L)=\omega(-L)=0$ $\left(\omega^{\prime}, \omega^{\prime \prime}\right.$ may not be continuous at $\left.x= \pm L\right)$, then we have

$$
u_{x x x}\left(x^{2}-L^{2}\right)=H\left(\omega_{x x}\left(x^{2}-L^{2}\right)\right) .
$$

Lemma A.3. Suppose $u_{x}=H \omega$. (a) We have

$$
\int_{\mathbb{R}} \frac{\left(u_{x}-u_{x}(0)\right) \omega}{x}=\frac{\pi}{2}\left(u_{x}^{2}(0)+\omega^{2}(0)\right) \geq 0 .
$$

In particular, A.6 vanishes if $u_{x}(0)=\omega(0)=0$.

(b) The Hardy inequality: Suppose that $\omega$ is odd and $\omega_{x}(0)=0$. For $p=2,4$, we have

$$
\int \frac{\left(u-u_{x}(0) x\right)^{2}}{|x|^{p+2}} \leq\left(\frac{2}{p+1}\right)^{2} \int \frac{\left(u_{x}-u_{x}(0)\right)^{2}}{|x|^{p}}=\left(\frac{2}{p+1}\right)^{2} \int \frac{\omega^{2}}{|x|^{p}}
$$

\section{Appendix B. NumericAl VAlues of some FUnCTIONS ON THE GRID POINTS}

We plot the numerical values of some functions on the grid points in this Section. We remark that all the estimates can be verified rigorously using the strategy we discussed in [1] (see page 21). The following figures are used to visualize several estimates.
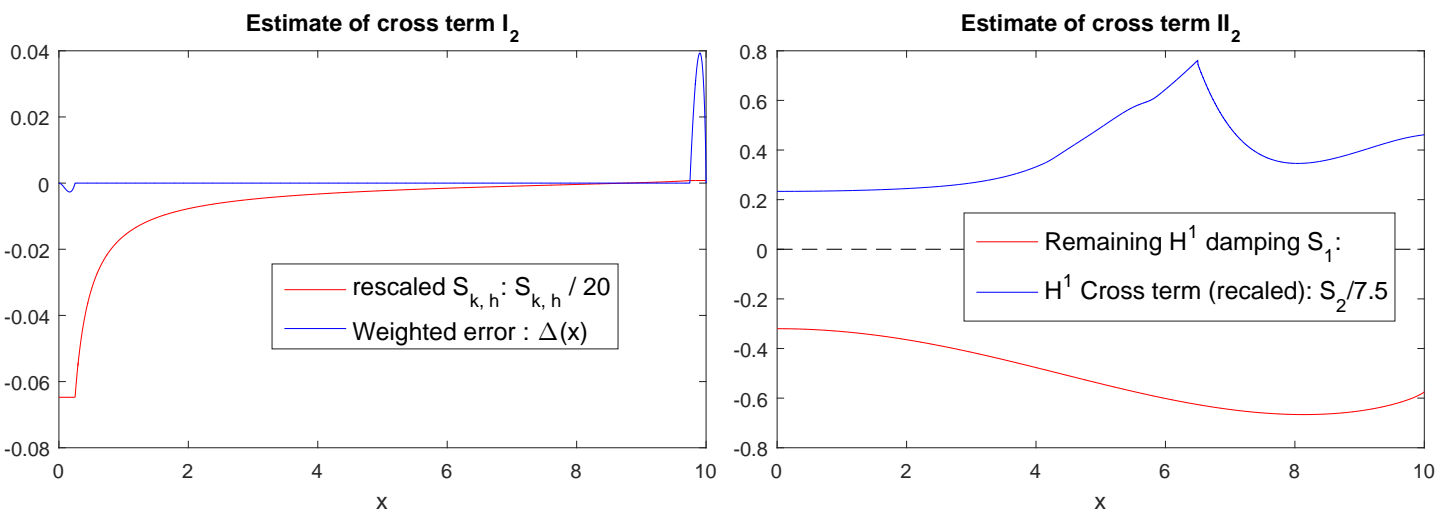

Figure 1. Left: Numerical values of $S_{k, h}$ and $\Delta(x)$ on the grid points. Right: Numerical values of $S_{1}$ and $S_{2}$ on the grid points. We plot the rescaled $S_{k, h}$, i.e. $S_{k, h} / 20$, and the rescaled $S_{2}$, i.e. $S_{2} / 7.5$.

B.1. Figure related to Section 2, On the left subfigure of Figure 1, the numerical values of $S_{k, h}$ are monotonically increasing and $|\Delta(x)| \leq 0.1$ on the grid points. On the right subfigure, the numerical values of $S_{1}, S_{2}$ on the grid points are less than $-0.3,7.5$, respectively. 

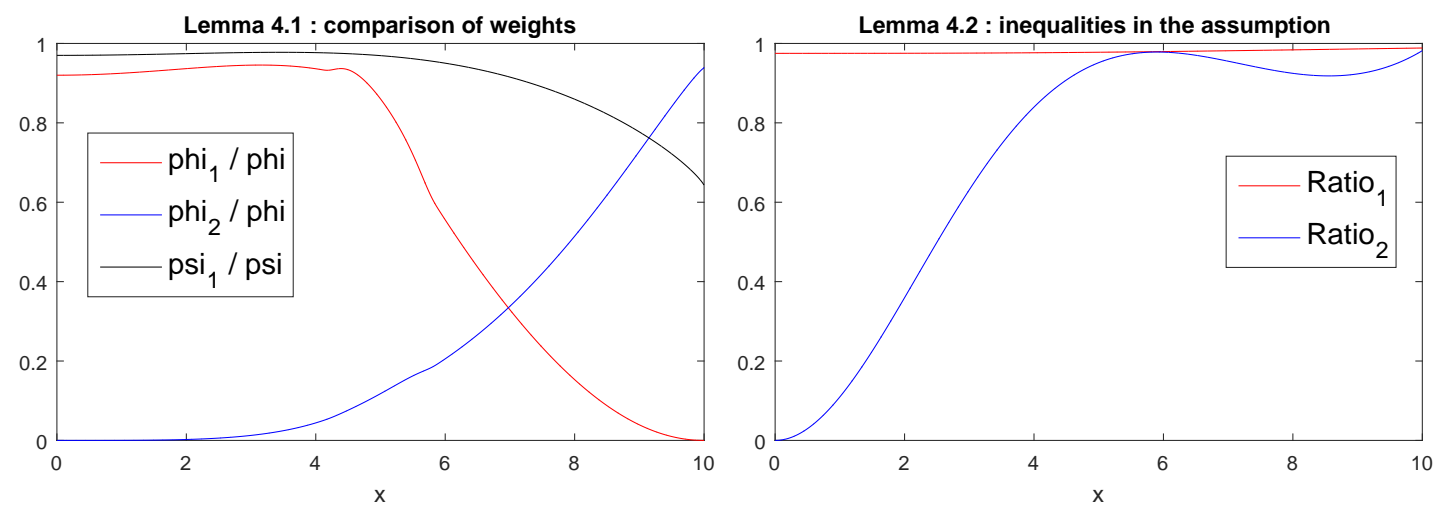

Figure 2. Comparison of several functions. Left: Numerical values of $\varphi_{1} / \varphi, \varphi_{2} / \varphi, \psi_{1} / \psi$ on the grid points. Right: Numerical values of two ratios in (4.4) on the grid points. Here, $\operatorname{Ratio}_{i}=\xi_{i}^{2}\left(\mu\left(\alpha_{i} \varphi_{i}-\xi_{i, x}\right) \alpha_{i} \psi_{i}\right)^{-1}, i=1,2$..

B.2. Figure related to Section 4, Numerical values of $\varphi_{1} / \varphi, \varphi_{2} / \varphi, \psi_{1} / \psi$ (see Lemma 4.1) and the ratio $\operatorname{Ratio}_{i}=\xi_{i}^{2}\left(\mu\left(\alpha_{i} \varphi_{i}-\xi_{i, x}\right) \alpha_{i} \psi_{i}\right)^{-1}, i=1,2$ (see Lemma 4.2) on the grid points are strictly less than 1 .

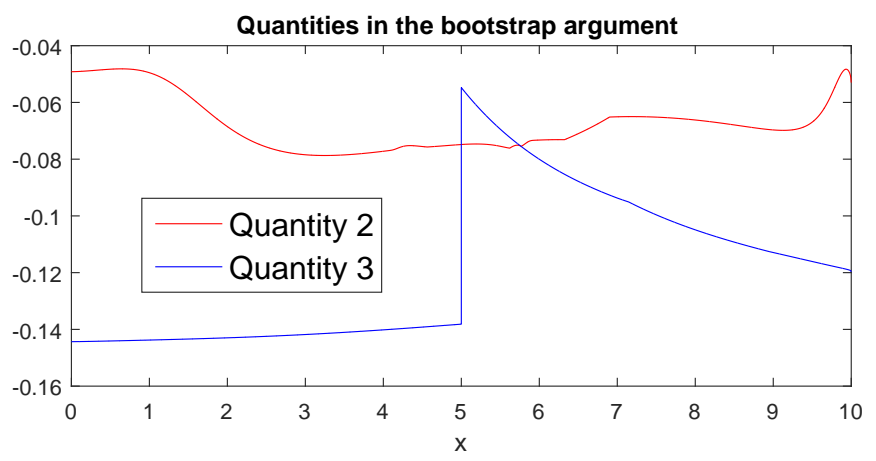

Figure 3. Numerical values of the left hand side of the second and the third inequality in (5.3) on the grid points.

B.3. Figure related to Section 5, Quantity 2,3 in Figure 3 represents the left hand side of the second and the third inequality in (5.3), i.e.

$$
\begin{aligned}
& \text { Quantity } 2 \triangleq-0.1+0.05 \varphi \rho_{1}^{-1}+Z_{1}(x) E^{*}, \\
& \text { Quantity } 3 \triangleq-0.2+0.05 \psi \rho_{2}^{-1}+\left(Z_{2}(x)+Z_{3}(x)\right) E^{*} .
\end{aligned}
$$

Numerical values of Quantity 2,3 on the grid points are less than $-0.04<0$.

\section{REFERENCES}

[1] Chen, J., Hou, T.Y., Huang, D. (2019). On the Finite Time Blowup of the De Gregorio Model for the 3D Euler Equation. preprint

Applied and Computational Mathematics, California Institute of Technology, Pasadena, Ca 91125, USA 